


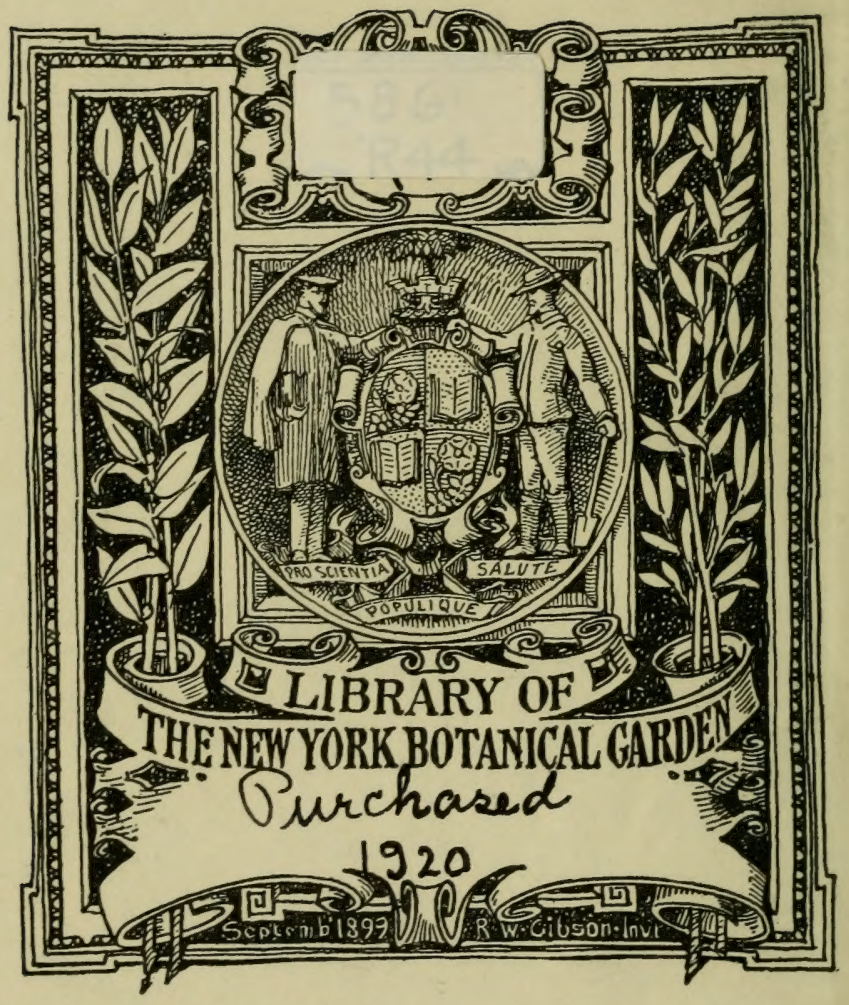








\title{
REPRODUCTION
}

\author{
DES
}

CRYPTOGAMES

PAR

\section{Maximilien RIETSCH}

Pharmacien supérieur,

Professeur suppléant à l'Ecole de pharmacie et de médecine de Marseille, Ex-pharmacien en chef des hospices civils de Marseille.

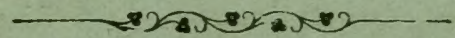

\section{P A R IS}

GERMER BAILLIERE, LIBRAIRE-ÉDITEUR 108, BOULEVARD SAINT-GERMAIN, 108

1882 

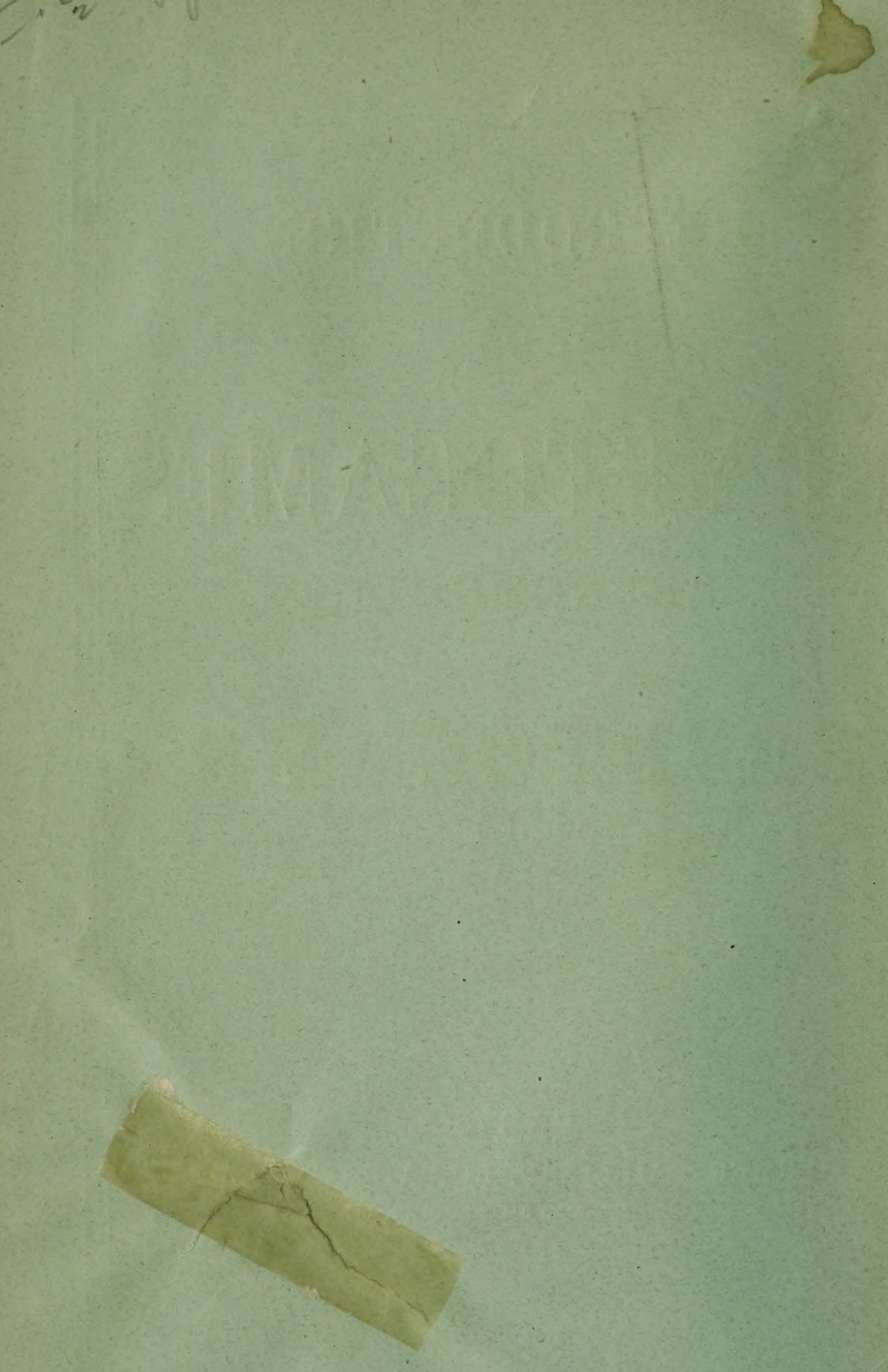

$\left.\frac{1}{8}\right)^{2}{ }^{2}$

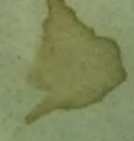

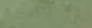

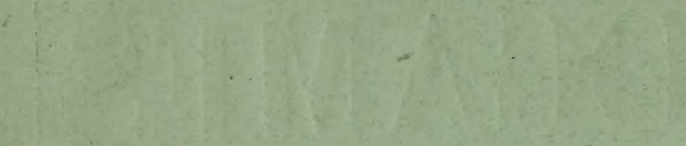

2 


\section{REPRODUCTION}

DES

CRYPTOGAMES 


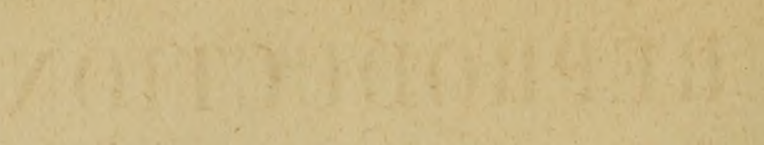




\section{REPRODUCTION}

DES

CRYPTOGAMES

PAR

\section{MAXIMILIEN RIETSCH}

Pharmacien supérieur,

Professeur suppléant à l'Ecole de pharmacie et de médecine de Marseille, Ex-pharmacien en chef des hospices civils de Marseille.

\section{P A R I S}

GERMER BAILLIERE, ÉDITEUR

108, BOULEVARD SAINT-GERMAIN, 108

1882 
4h5? 


\section{REPRODUCTION}

\section{DBS \\ CRYPTOGAMES}

\section{INTRODUCTION.}

On rencontre dans les eaux douces et marines des êtres d'une organisation extrêmement simple (Protogenes, Protomoeba), consistant en une petite masse de protoplasma homogène sans noyau; leur forme est indéterminée; ils émettent des prolongements lobés qu'ils rétractent ensuite et à l'aide desquels ils se déplacent; ils englobent les particules alimentaires qu'ils peuvent rencontrer. Ainsi nourris, ils grandissent; puis, quand leur corps a atteint un certain volume, un étranglement apparaît à la périphérie et se prolonge peu à peu jusqu'au centre, divisant le petit être en deux portions égales ou inégales, qui lui ressemblent entièrement, et qui continuent à vivre de la même façon. C'est le mode de reproduction le plus simple que l'on connaisse : ainsi la multiplication apparaît comme une conséquence de la nutrition et de l'accroissement; elle devient plus complexe à mesure que l'on envisage des êtres plus différenciés. La forme, en effet, devient fixe, les pseudopodes se changent en cils, le protoplasma peut acquérir un noyau, une membrane, de la chlorophylle, les individus s'associent en colonies, etc.; toutes ces transformations, dont plusieurs peuvent apparaître en même temps, tendent à modifier le mode primitif de multiplication. Ces transformations s'accentuent finalement dans deux directions divergentes, conduisant aux règnes 
animal et végétal; mais à l'origine, cette différence est peu marquée, et on peut considérer les Monères comme un centre, vers lesquels convergent un certain nombre de séries rayonnantes, d'autant plus semblables entre elles, qu'on les compare à une moindre distance du point de départ commun. Dans les séries végétales, les unes conduisent aux champignons, ce sont les Myxomycètes et les Bactériens, auxquels se rattacheront les Saccharomycètes; les autres aux Algues: ce sont, par exemple, les Cyanophycées, si étroitement parentes des Bactériens.

Dans ces trois séries, la multiplication par scission existe encore, mais déjà plus complexe; le bourgeonnement, la formation des spores peuvent être considérés comme dérivant plus ou moins directement du mode de division primitif. Mais déjà dans les Myxomycètes nous rencontrons un phénomène en apparence inverse : c'est la fusion d'un ou de plusieurs éléments en une cellule unique. Cette fusion entre individus entièrement semblables ne semble avoir d'autre effet que d'augmenter leur masse ; néanmoins c'est là, sans doute, l'origine de la sexualité. Dans le vaste groupe des Algues dont les Chiorophycées font dejà partie, nous pourrons, en effet, passer par une série de transitions; de la fusion de cellules entièrement semblables, au moins en apparence. à la véritable fécondation, c'est-à. dire à la copulation entre deux éléments dissemblables; de cette copulation semble résulter une impulsion nouvelle dans le développement, une augmentation d'énergie. La conséquence immédiate est la formation d'un $\propto u f$, point de départ d'une génération nouvelle; dans les Algues, la fécordation se perfectionne à mesure que l'on remonte la série; dans les Champignons c'est la marche inverse que nous constaterons.

Mais même dans les Algues la fécondation, en général, n'apparaît pas régulièrement, et souvent elle semble seulement la conséquence de certaines circonstances extérieures ; la multiplıcation assurée prédomine. Dans les végétaux terrestres, les Mousses et les Cryptogames vasculaires, l'acte sexuel reparaît périodiquement; de là une alternance de génération beaucoup plus régulière que dans les Algues; en même temps la propagation végétative devient moins importante, ce qui fait prévoir déjà le rôle très subordonné auquel elle se trouve réduite dans les végétaux supérieurs. 


\section{MYXOMYCETES.}

Les Myxomycètes (MM. Baranetzki, de Bary, Brefeld, Cienkowski, Tamintzni et Woronine, Rostafinski, Rose, Wigand), pendant une partie de leur existence, se comportent absolument comme certains protistes; d'un autre côté, ils ont des affinités évidentes avec les Champignons.

Par cette double parenté, ils peuvent servir d'intermédiaires des premiers aux seconds.

On peut considérer comme l'individu le plus simple de ce groupe, le Plasmodium Brassica, décrit par M. Woronine; c'est une petite masse plasmique, qui jamais ne s'entoure d'une membrane; mais se résout simplement en un certain nombre de masses plus petites, amiboïdes; chacun de ces myxomoebes pénètre dans la racine du chou, où, seul ou par fusion avec des congénères, il finit par constituer une plasmodie nouvelle.

Les autres Myxomycètes s'entourent toujours à un moment donné d'une membrane, afin de former leurs spores. Ceux-ci peuvent conserver pendant des années leur faculté germinative. Leur membrane, ordirairement colorée, lisse ou ponctuée, se déchire à la germination; le protoplasma s'échappe en une seule ou en plusieurs petites masses dont chacune constitue un amibe nucléé, se mouvant par ses pseudopodes (myxamoebe) et contenant une à trois vésicules pulsatiles. Ces amibes se déplacent sur les détritus végétaux dont ils se nourrissent; ils s'allongent bientôt, prennent un long cil vibratile et se transforment en zoospores, qui rampent encore sur le substratum ou nagent dans l'eau. Zoospores et amibes se reproduisent par bipartitions, et, après avoir retracté leur cil ou leurs pseudopodes, ils peuvent s'enkyster, quand les circonstances deviennent défavorables, et donner alors une spore qui se comportera comme la spore primitive.

Mais ces cellules nues et mobiles peuvent aussi se réunır par copu- 
lation au nombre de deux ou plusieurs, constituant ainsi une plasmo. die diffluente qui rampe, s'étale en réseau, augmente de volume par nutrition et aussi par l'adjonction de nouvelles zoospores. Ces plasmodies, qui peuvent s'étaler en réseau, se dessécher pour se transformer en sclérote, se conjuguer avec d'autres plasmodies, et acquérir souvent un volume considérable (Ethalium), finissent toujours par former un ou plusieurs sporanges. Ceux-ci ont souvent un développement fort rapide; ils apparaissent sur les plasmodies comme autant de protubérances dans lesquelles passe peu à peu tout le protoplasma, en se débarrassant des corps étrangers qu'il pouvait tenir englobés jusque-là. Il se différencie en pied et en sporange ; le premier, étalé à la base, soudé souvent avec les pieds voisins, se prolongeant parfois en columelle (Stémonitées) dans la cavité du sporange. Celui-ci se compose d'une membrane mince, fragile, incrustée de calcaire, souvent de couleur vive, à surface lisse ou garnie de proéminences; elle entoure la masse pulvérente des spores qui remplissent à eux seuls toute la cavité du sporange (Libraria, Licea) ou qui sont accompagnées d'un capillitium. Celui-ci se compose de tubes isolés, fusiformes, à épaississement spiralé (Trichia), ou de filaments anastomosés portant extérieurement des anneaux (Arcyria), ou d'un réseau de tubes minces, renflés aux nœuds où ils contiennent des granulations calcaires (Physarum). Le fruit, en forme de gateau, de l'Ethalium, est plus compliqué, et peut être considéré comme un enchevêtrement de sporanges tubuliformes. Rarement les sporanges sont dépourvus de membrane (Dictyostellum); dans les Exosporées (Ceratia, Polystica), c'est à leur surface extérieure qu'ils portent les spores sur de courts stérigmates. A la maturité, la membrane se détruit irrégulièrement ou se déchire; le capillitium se redresse et dissémine les spores.

Celles-ci sont très petites, arrondies, lisses ou hérissées de verrues ou 'munies de bandes réticulées.

Myxomycìtes agrégés(MM. Cienkowski, Van Tieghem).-Au lieu de se fusionner en plasmodie, les amibes peuvent s'agréger tout simplement en un massif cellulaire; en glissant les unes sur les autres, elles constituent alors un cône dressé en l'air, dont les cellules axiles, plus grosses, s'entourent d'une membrane et se changent en pied, pendant 
que les cellules extérieures se superposent par glissement au sommet du pied pour $y$ former un chapelet ou un pinceau de spores arrondies, entourées chacune d'une membrane cuticularisée et échinée (Acraria granulata). D'autres fois les spores restent réunies par une substance gélatineuse en une petite masse (Dictyostelium). Les amibes s'enkystent encore, quand les circonstances sont défavorables; dans le dernier genre cité, ils donnent, à ce moment, par bourgeonnement, plusieurs spores enkystés (M. Van Tieghem). D’après M. Brefeld, il y aurait dans le $D$. mucoroïdes une fusion instantanée au moment de la montée du fruit, suivie aussitôt d'une division en portions de même grandeur. Le cas serait donc intermédiaire entre les Myxomycètes ordinaires et les agrégrés.

M. Sorokine a signalé, dans le Bursulla crystallina, la coexistence de deux espèces de spores, les unes nucléeés, les autres sans noyau; elles copuleraient entre elles, et ce serait là un premier indice de sexualité ; le résultat est une oospore; celle-ci germe au printemps en donnant un sporange pédicellé, dont les zoospores sont dépourvus de noyau.

\section{SCHIZOMYCĖTES OU BACTÉRIES.}

Les Bactéries sont des êtres extrêmement simples, unicellulaires, dépourvus de noyaux, mais munis d'une membrane comme les cellules végétales; leurs dimensions variables sont, en général, des plus réduites. Elles sont douées de motilité et vivent isolées ou réunies en chapelets (torula), en filaments (leptothrix); elles peuvent encore se rassembler en colonies, dans l'intérieur desquelles elles sont libres (essaim) ou soudées ensemble par une substance glaireuse (zooglcea).

Jusqu'à présent on ne connaît point de reproduction sexuée dans les Bactéries; elles ne semblent se multiplier que par scissiparité et spores endogènes.

Scissiparité. - En dehors des Sarcina qui se segmentent par des cloisons perpendiculaires dans les trois directions, et qui constituent 
ainsi des familles cubiques de 4 à 16 cellules ou plus, la scissiparité consiste en un fractionnement transversal de la cellule. Celle-ci s'allonge à peu près du double, son protoplasma s'éclaircit, suivant une ligne médiane perpendiculaire aux deux faces latérales, puis une cloison apparaît au même endroit, divisant la cellule en deux. La cloison s'èpaissit, la lamelle moyenne se gélifie et sépare ainsi les deux articles. Ce disque gélatineux peut se distendre davantage, se dissoudre; les deux articles deviennent alors libres, entourés chacun d'un étui gélatineux. Mais quand cette bipartition a lieu pendant le mouvement de la bactérie, le disque gélatineux peut aussi s'étirer par traction en un long fil qui finit par se rompre, et chaque article se trouve alors muni d'ur prolongement effilé, simulant un cil. Il peut y avoir un pareil cil à une seule extrémité ou aux deux; ce cil peut aussi se fendre longitudinalement, et alors il y aura plusieurs cils (Bacillus amylobacter (1), M. Van Tieghem).

Le phénomène se passe d'une façon analogue dans les Spirillum qui s'allongent, avant de se segmenter, de façon à former à peu près quatre tours de spire; les deux moitiés s'inclinent souvent l'une vers l'autre, le disque gélatineux servant de charnière ; elles peuvent alors s'enchevêtrer. Dans les Spirochaete, il n'est pas possible de voir les cloisons transversales, même sur des filaments allongés, de façon à former huit tours de spire; les articles, courts et au noinbre de quatre pour chaque tour, ne deviennent visibles qu'au moment de la formation des spores.

Le sectionnement peut être précédé d'un étranglement vers le milieu de la cellule; la bactérie paraîtra alors souvent constituée par une cellule renflée aux deux extrémités, comme un 8 de chiffre.

La scission peut aller plus vite que la séparation des cellules qui restent alors réunies en filaments plus ou moins longs; ceux-ci s'enchevêtrent irrégulièrement ou sécrétent un mucus qui les relie, ou bien encore se dissocient en bâtonnets mobiles ou immobiles (Bacillus subtilis). Pour la formation des essaims et zooglœæ, les deux ar-

(1) Bacillus amylobacter, ferment butyrique, très ressemblant au Bacillus subtilis qui ne produit aucune fermentation. 
ticles résultant d'un bâtonnet, glissent l'un sur l'autre et viennent se juxtaposer, et ainsi de suite; il se forme d'abord une surface composée de bâtonnets parallèles, ou plus ou moins obliques, puis, peu à peu, une masse compacte, provenant souvent tout entière d'un bâtonnet unique; les articles composant peuvent, du reste, être libres ou réunis par une substance gélatineuse. Dans le Leuconostoc mesenterö̈des, les cellules séparées restent disposées en chapelets, entourés d'une matière glaireuse (M. Van Tieghem).

La rapidité de cette multiplication dépend directement de la richesse nutritive du milieu; elle est active, tant que celur-ci n'est pas épuisé; mais, ce résultat une fois atteint, les bactéries tombent au fond et demeurent immobiles. La température est aussi un facteur très important; l'optimum varie beaucoup pour les différentes Bactéries. Il en est qui se multiplient encore à $69-70^{\circ}$ (M. Miquel), même à 740 (M. Van Tieghem). Toutes les bactéries meurent cependant audessous de $100^{\circ}$; au voisinage de $0^{\circ}$, la multiplication cesse complètement, mais la Bactérie ne meure pas pour cela. En s'écartant de l'optimum, la multiplication devient moins rapide; voici quelques chiffres donnés par M. Brefeld pour le Bacillus subtilis : un bâtonnet se divise en une demi-heure à $24^{\circ}$ Réaumur, en trois quarts d'heure à $25^{\circ}$, en une heure et demie à $15^{\circ}$, en quatre ou cinq heures à $10^{\circ}$, très lentement, à $5^{\circ}$. D'après M. Eidam, le Bacterium Termo commence à se segmenter à $5^{\circ} 5 \mathrm{C}$., optimum entre 30 à $35^{\circ}$, à $40^{\circ}$ la segmentation s'arrête.

La multiplication ou plutôt la vie des bactéries est la cause déterminante de nombreuses fermentations : fermentation acétique, provoquée par le Micrococcus aceti, nitrique (M. nitrificans), butyrique (Bacillus amylobacter), etc.

Formation des spores. - Elle a été constatée dans des cas nombreux et chez des Schizomycètes assez divers, pour qu'on doive l'admettre aujourd'hui comme générale (MiM. Robin, Pasteur, Van Tieghem, Cohn, Billroth, Koch, Brefeld, Praznowski). C'est quand les conditions de milieu deviennent défavorables à la multiplication par scissiparité, quand le liquide nutritif s'épuise, que l'on voit apparaître 
les spores; la présence de l'oxygène semble indıspensable dans beaucoup de cas. Les spores, nommées aussi corpuscules, germes, etc, ont éré fréquemment prises pour des espèces spéciales, et rangées par les Micrococcus qui paraissent en l'éalité moins nombreux qu'on ne l'avait cru d'abord.

Dans le Bacillus subtilis, c'est ordinairement au milieu, plus rarement à l'extrêmité du bâtonnet que l'on voit apparaître des points plus brillants indiquant une condensation de protoplasma; il se concentre bientiot tout entier en une masse oblongue, cylindrique, fortement réfringente, qui s'entoure d'une membrane, constituant ainsi la spore. Le bâtonnet se renfle alors dans la région correspondante; enfin sa membrane finit par être résorbée. Dans les bâtonnets composant un même filament, on peut rencontrer les spores à divers états de développement. L'apparence sombre de leur noyau a été attribuée par M. Cohn à des corps gras; M. Brefeld ne partage pas cette manière de voir.

Dans le Bacillus amylobacter, certains bâtonnets cessant de s'allonger, prennent une réfringence différente et une forme de tétard, de fuseau ou d'ellipsoïde; une ou plusieurs gouttelettes brillantes apparaissent dans leur intérieur, puis se réurissent en une goutte unique, autour de laquelle s'amasse tout le protoplasma du bâtonnet, de façon à constituer une spore ovale ou cylindrique. C'est dans les bâtonnets immobiles, quelquefois cependant aussi pendant leur mouvement, que les spores se forment (MM. Van Tieghem, Praznowski.)

Le Bacterium lucens produit ses spores, quand on le place dans l'eau pure; ses articles, étranglés au milieu, forment une spore dans une des deux moitiés, pendant que l'autre se vide; quelquefois aussi, chaque moitié montre une spore et une cloison semble alors se constituer au milieu du bâtonnet (M. Van Tieghem). La bactérie de la clavelée (M. Toussaint) montre presque toujours la réunion de deux bâtonnets inégaux; le plus long donne ordinairement une spore à chacune de ses extrémités, quelquefois une troisiéme en son milieu; le plus petit n'en donne quelquefois qu'une seule, et prend alors une forme de massue; car la spore possède un diamètre supérieur au sien ; la spore est également ovale et très réfringente. Le Bacillus crassus 
(M. Van Tieghem) est remarquable parle grand volume de ses spores; elles mesurent $0^{\mathrm{mm}}$. 005 ; il s'en forme une dans chaque article à l'une de ses extrémités. Un autre Bacillus, appelé Dispora caucasica par M.Kern, qui l'a trouvé dans le lait fermenté, développe dans chaque bàtonnet, deux spores situées à ses deux extrêmités. On voit donc que dans les micro et dermo-bactéries le nombre des spores qui prennent naissance dans chaque article est variable, et qu'elles apparaissent tantôt dans le renflement terminal de l'article, tantôt en son milieu, tantôt dans une région variable.

Quand le Spirillum amyliferum (M. Van Tieghem) s'apprête à don. ner des spores, ses filaments forment ordinairement deux spires, cescent de s'allonger, grossissent, prennent une réfringence différente, ne se colorent plus par l'iode en jaune, mais bien en bleu, sauf cependant dans deux régions qui restent blanches et qui sont placées ordinairement aux deux extrémités du filament; quelquefois aussi l'une d'elles est an milieu. Si l'article ne fait qu'un tcur, il possède une seule région blanche terminale. Ces régions indiquent les points de formation des spores futures; celles-ci apparaissent bientôt brillantes, à contours sombres, à membrane cutinisée. Plus tard, le filament cesse de bleuir par l'iode; un liquide hyalin remplit sa cavité; une cloison médiane a pris naissance dans les articles à deux tours de spire; chaque spore se trouve donc alors située dans une cellule distincte et s'appuie, soit contre cette cloison médiane, soit contre une des cloisons extrêmes. Plus tard, la membrane de l'article est résorbée et la spore devient libre. Dans les Spirochaete, chaque cellule produit encore une spore; un filament à huit tours de spire, en possède donc à peu près 32. Le Vibrio serpens forme aussi les siennes dans l'eau pure, en se découpant en 4 à 6 articles, chacun avec une spore.

Le Leuconostoc produit ses spores d'une tout autre manière: quand les circonstances deviennent défavorables à son développement ordinaire, certaines de ses cellules grandissent, épaississent leur paroi et se transforment directement chacune en une spore qui possède un grand pouvoir résistant. Cette bactérie qui intervertit le sucre de canne et absorbe le glucose et le lévulose pour les convertir en hydra. tes de carbone gélatineux, exerce de grands dégâts dans les fabriques 
de sucre. Elle a été étudiée par M. Van Tieghem qui a montré qu'clle se rapprochait beaucoup des Oscillariées dont elle diffère par l'absence de chlorophylle et qu'elle constituait en réalité une forme de transition entre celles-ci et les Schizomycètes.

La formation, non plus de spores endogènes, mais de conidies, a été signalée par M. Engel dans le Bacillus puerperalis, puis dans le leptothrix; plus tard aussi par M. Koch. M. Toussaint, en cultivant la bactéridie charbonneuse, dans le sérum du sang de chien et dans la chambre chaude de $\mathbf{M}$. Ranvier, a vu les filaments prendre un diamètre transversal presque double du diamètre ordinaire; puis le protoplasma s'amasser en certains points plns réfringents qui, augmentant considérablement de volume, forment des organes ovoïdes plus ou moins allongés ou bien renflés en boule ou en gourde; dans l'intérieur de ces sporanges se forment ensuite 3 à 6 spores nettes et réfringentes.

Malgré ces observations, on peut seulement considérer comme phénomène général, la formation des spores endogènes; $M$. Brefeld a donné quelques détails intéressants sur le temps nécessaire à cette formation dans le Bacillus subtitis : à $24^{\circ}$ Réaumur, il faut 12 heures, à $18^{\circ}, 24$ heures, à $15^{\circ}, 48$ heures, à $10^{\circ}$, plusieurs jours; au-dessous de $5^{\circ}$, les spores ne se forment plus. M. Pasteur a fait voir aussi qu'il y a un maximum de température au-dessus duquel il ne se produit plus de spores, quoique les bactéries continuent à se multiplier.

Germination des spores. - Les spores du bacillus subtilis (MM. Brefeld, Cohn, Koch, Praznowski) peuvent germer de suite après leur formation; la germination exige un demi jour au plus à la température ordinaire; elle est hâtée par une augmentation de température, plus encore par une ébullition de cinq minutes du liquide nutritif; en laissant refroidir lentement celui-ci, la germination a lieu en deux ou trois heures. La spore, un peu oblongue, perd d'akord son aspect sombre; l'aréole qui l'entourait disparaît; elle augmente de volume; à son centre, on distingue une zone cläire et réfringente qui s'agrandit bientôt; la spore perd ses contours réguliers. Sur un de ses côtés longitudinaux un refoulement se fait de dedans en dehors; le proto- 
plasme s'accumule dans la pointe; là la membrane extérieure de la spore s'ouvre; le germe sort, s'allonge en bàtonnet; sa région postérieure reste incluse dans la membrane de la spore. La perforation a lieu exactement au milieu de la spore allongée dont la membrane apparaît alors plus épaisse aux deux pôles; cette membrane semble correspondre à l'exospore; elle adhère souvent assez longtemps au bâtonnet, même quand il s'est déjà augmentẻ et déplacé; elle finit cependant par se détacher. L'orifice de sortie paraît alors arrondi, à bords retroussés de dedans en dehors. Tout le contenu de la spore passe dans le bâtonnet qui est perpendiculaire à l'axe longitudinal de la spore et par suite à celui de lá cellule mère.

Le Bacillus amylobacter (MM. Van Tieghem, Praznowski) diffère du précédent par ses bâtonnets deux fois plus longs et plus épais, mais ce caractère ne semble pas très constant. La germination établit une différence moins variable. Le tube germinatif du Bacillus amylobacter perfore en effet l'exospore, non au milieu, mais à un des pôles de la spore; l'exospore est résorbée dans la région correspondante; il se forme ainsi un orifice ovale. L'extrémité postérieure du filament s'écarte de suite de la membrane externe qui présente partont la même épaisseur et qui reste moins longtemps adhérente. Les spores du $B a$ cillus anthracis se divisent en quatre cellules filles dont chacune donne un bâtonnet (M. Ewart). M. Van Tieghem a observé la germination chez les Spirillum: la spore grossit, l'exospore se trouve rompue; il en sort un tube qui s'allonge et se recourbe aussitôt en hélice; quand celle-ci possède deux tours de spire, elle acquiert une cloison médiane; l'allongement continue; bientôt la spire présente quatre tours avec trois cloisons; alors ordinairement elle se sépare en deux.

M. Brefeld a constaté qu'il était facile d'empêcher la germination des spores au moyen des acides; il suffit pour cela que le liquide nutritif soit additionné: de 1/2000 d'acide sulfurique, chlorhydrique, azotique, tartrique ou citrique 1/500 d'acide butyrique, 1/300 d'acidé acétique. Les acides phénique et salicylique sont moins actifs. Il faut $1 / 500$ d'ammoniaque.

Des solutions sentant déjà fortement l'ammoniaque ou l'acide phénique n'empêchent pas la germination, ni les mouvements des bâton- 
nets. La culture préalable dans des liquides faiblement acides diminue notablement l'influence de ces agents sur la germination. Les autres bactéries semblent moins sensibles; cependant $1 / 100$ d'acide minéral suffit toujours pour arrêter le développement.

Il est bien entendu que, dans tous les cas précédents, les spores ne sont pas tuées.

La reproduction des bactéries se présente donc avec les caractères d'une extrême simplicité; ce quilui donne un cachet tout particulier, c'est que d'abord les bactéries sont extraordinairement prolifiques, toutes les fois qu'elles rencontrent un milieu favorable; en second lieu,les spores offrent aux agents destructeurs une résistance bien supérieure à celle de toutes les autres cellules végétales.

Vitesse de reproduction. - En supposant qu'une bactérie se divise en deux dans l'espace d'une heure, et nous avons vu que cette hypothèse était au-dessous de la réalité (dans certaines conditions), M. Cohn a fait les calculs suivants: en 24 heures, le nombre des bactéries s'élèvera à plus de 16 millions et demi, en deux jours à 281 milliards, en trois jours à 47 trillions; en cinqjours, malgré leur extrême petitesse, tout l'espace occupé par les mers pourrait être rempli.

Résistance et ubiquité des spores. - M. Pasteur a tout d'abord appelé l'attention sur cette extraordinaire vitalité des spores dont, avant ses remarquables travaux, on n'avait aucune idée. Il est bien démontré aujourd'hui que l'ébullition dans l'eau qui détruit tous les êtres vivants, n'atteint pas les spores de nombreuses bactéries, à moins qu'elle ne soit longtemps prolongée. Il est nécessaire de porter la température à $110^{\circ}$ et même $115^{\circ}$, surtout quand les liquides sont alcalins; l'acidité diminue la resistance: les spores sèches ne sont tuées sûrement que par une température de $150^{\circ}$. Cependant l'ébullition à l'air libre peut suffire, même dans les liqueurs alcalines, le lait, par exemple; mais à la condition d'être répétée deux fcis à un jour de distance; dans l'intervalle en effet, les spores se sont développées en bâtonnets dont le pouvoir résistant est bien moindre (M. Duclaux). De ce qu'une liqueur acide, après avoir été chauffée, ne montre point de 
germination, il n'en résulte pas qu'elle se trouve stérilisée; l'acidité, en effet, s'oppose à la germination des spores. On ne sera certain du résultat, que si la germination n'apparait pas, même après neutralisation ou culture dans un autre liquide nutritif.

M. Toussaint a constaté que, chez les animaux tuberculeux, tous les liquides de l'économie sont virulents; maintenus quelque temps à $55-58^{\circ}$, ils ne deviennent pas inoffensifs. La chair cuite à jus rouge, comme une còtelette, puis exprimée, donne un liquide qui, injecté à des lapins, a amené leur mort en cent vingt jours; l'ingestion de la viande a produit un résultat plus rapide encore.

D’après M. Cohn, certaines spores de Bacillus endurent une température de 70 à $80^{\circ}$ pendant trois à quatre jours; d'après M. Frisch, un froid dépassant $-87^{\circ}$ ou même $-111^{\circ}$ ne les tue pas.

M. Brefeld a constaté que, pour le Bacillus subtilis, une demi-heure d'ébullition ne fait que favoriser la germination des spores; une heure d'ébullition diminue la faculté germinative. Pour stériliser la liqueur, il faut la maintenir trois heures en ébuilition, ou un quar't d'heure à $105^{\circ}$, dix minutes à $1117^{\circ}$, cinq minutes à $110^{\circ}$. L'action combinée de la chaleur et des acides tue mieux les spores; pour la vérification des résultats, il faut se conformer aux précautions indiquées plus haut. La stérilisation peut être amenée par une pression de vingt-trois à vingt-quatre atmosphères d'oxygène (M. Paul Bert); douze atmosphèr'es seraient suffisantes pour le Bacillus anthracis (M. Ewart). Les spores de Bacillus subtilis résistent à des solutions concentrées de sulfate de cuivre, de bichlorure de mercure, d'acide phénique. Après un an passé dans l'eau, puis trois ans à sec, elles n'ont point peráleur faculté germinative (M. Brefeld). M. Pasteur a montré que les spores du vibrion de la septicémie et celles de la bactérie du charbon étaient encore viables après douze ans et plus. Cette résistance extraordinaire aux agents de destruction, leur extrème petitesse, les circonstances favorables à leur développement qu'elles rencontrent très fréquem ment, font qu'il existe presque partout des spores diverses; l'air, l'eau surtout en charrient constamment; elles se déposent sur tous les corps solides; la poussière de nos rues et de nos appartements en cst infectée (MM. Pasteur, Miquel, Tyndal). Les hactéries jouent un grand Rietsch. 
rôle dans la physiologie générale; elles rendent beaucoup plus rapide le retour à la nature minérale des cadavres animaux et végétaux; mais elles ne dédaignent pas les êtres vivants. Pour certaines maladies contagieuses il est démontré, pour d'autres il est très probable, que les bactéries sont leur cause unique; elles trouvent, en effet, dans le sang un terrain très propice à leur prodigieuse multiplication, elles amènent ainsi la mort de la victime dont le cadavre et les déjections deviennent de nouvelles sources d'infection. Ce qui peut donc paraître étonnant, c'est que ces maladies ne soient pas plus fréquentes, leurs victimes plus nombreuses. Mais les téguments constituent aux organismes vivants une protection très efficace, les bronches et leurs ramifications agissent à peu près comme un tampon de coton ( $M$. Tyndall), et le tube digestif n'est pas un milieu très favorable au développement de la plupart des bacteries ni à leur introduction dans le sang; pour beaucoup de ces petits êtres l'air libre est un agent d'oxydation beaucoup trop énergique; ils ne se propagent activement que sous des influences oxydantes beaucoup plus faibles; enfin, les cellules vivantes offrent une certaine résistance qui retarde ou arrête même leur développement. De là il résulte immédiatement que l'immunité des animaux, de l'homme particulièrement, sera variable avec la constitution, les dispositions héréditaires, momentanées, etc. Les considérations précédentes montrent aussi par exemple comment des maladies, en réalité contagieuses, ont pu pendant longtemps être considérées comme héréditaires.

C'est surtout aux remarquables travaux de M. Pasteur qu'est due l'introduction dans la science de ces idées nouvelles; elles ont déjà été fécondes en heureax résultats. Mais il s'en faut qu'elles aient été acceptées d'emblée par tout le monde; et à la panspermie on a souvent opposé l'hétéroyénie, c'est-à-dire la création des bactéries de toutes pièces au moyen de substances minérales ou organiques, ou organisées; dans ce dernier cas l'hétérogénie revient à la transformation en bactérie d'une cellule vivante appartenant à un autre être, c'est-àdire au polymorphisme.

Quant à cetie dernière théorie, il est suffisamment prouvé aujourd'hui que certains Schizomycètes et leurs spores peuvent exister et 
existent à l'intérieur d'organismes vivants ct en bonne santé; d'un autre côté, la transformation instantanée en bactérie d'une cellule vivante appartenant à un animal supérieur, n'est guère conciliable avec l'état actuel de nos connaissances en sciences naturelles. L'hétérogénie au moyan de substances minérales ou organisées, revient à la génération spontanée; la synthèse, c'est-à-dire la création de toutes pièces d'une matière albuminoïde, ne tardera pas sans doute à être devenue une rẻalité. Mais le protoplasma vivant n'est pas une simple matière albuminoïde; nous savons fort peu de chose sur sa constitution; cependant, il est permis de supposer qu'il est au moins une com binaison de matières albuminoïdes diverses et de corps gras. Nous savons d'ailleurs que de pareilles combinaisons exigent un temps d'autant plus long que la molécule tinale est plus complexe; l'élévation de température ne pourra être employéeque dans des limites très restreintes pour hater la réaction; il est donc présumable que, si les diverses substances protéiques récessaires se trouvaient réunies, il leur faudrait de nombreuses années pour se combiner, se grouper, s'orienter de façon à produire cette résultante : le protoplasma vivant. En supposant mêmeque le vase en expérience se trouve, pendant ce long espace de temps, placé dans ies conditions voulues de température, d'accès de l'air, etc., conditions sur lesquelles notre ignorance est à peu près aussi complète que sur la constitution du protoplasma; en le supposant préservé surtout de l'invasion des bactéries, il ne faudrait pas s'attendre à voir apparaitre la vie sous une autre forme que celle d'un monère, d'un protoplasma diffluent. Les bactéries sont déjà relativement des êtres complexes; leur forme définie, leur membrane, leurs modes de reproduction impliquent une origine ancienne, et leur apparition brusque ne semble pas actuellement pouvoir être interprétée en faveur de l'hétérogénie. Cela ne prouve rien évidemment, quant à l'origine première de la vie sur la terre; nous sommes loin de pouvoir reproduire dans nos laboratoires les conditions alors réalisées.

Des travaux recents ont démontré que les bactéries sont susceptibles d'éprouver certaines transformations physiologiques. En cultivant la bactérie très virulente du charbon (Bacillus anthracis) dans du bouillon de poule, en présence de l'air et à une température de 42 à 
$43^{\circ}$, M. Pasteur a pu atténuer successivement leur virulence jusqu'à la rendre nulle; cette atténuation semble résulter d'un état maladif, car la bactérie finit par mourir. Inoculée peu avant ce résultat extrême, elle n'occasionne aucun désordre, bien plus, elle devient un préservatif, un vaccin, contre une génération moins atténuée; en opérant plusieurs vaccines avec des bactéries de plus en plus virulentes, l'individu finit par acquérir une immunité complète contre le charbon. Il est nécessaire de débarrasser le liquide des spores ou d'empêcher leur formation; car elles ne subissent pas la modification indiquée; c'est le second résultat qui se trouve réalisé dans l'expérience précédente. Par des cultures répétées sur des animaux vivants, la bactérie reprend au contraire une virulence croissante. Tels sont les résultats remarquables et féconds en conséquences, obtenus par M. Pasteur qui attribue l'atténuation à l'influence de l'oxygène. Une température de 85 à. 100. sémble produire à l'air des résultats semblables, plus rapidement, mais moins sûrement (MM. A rloing, Cornevin, Thomas).

D'après M. Büchner, on peut transformer le Bacillus anthracis en bactérie du foin non virulente par des cultures dans un liquide spécial et en présence de l'air. Une température de $25^{\circ}$, l'absence de l'oxygène, une réaction faiblement acide, toutes les causes en un mot qui empêchent ou retardent la multiplication par scissiparité, tendent à maintenir la virulence. La culture en couche faible, l'agitation du vase, en facilitant l'accès de l'oxygène, sont favorables à la multiplication, défavorables par conséquent à la virulence; l'élévation de la température au-dessus de $25^{\circ}$ agit dans le mêrne sens; M. Büchner a même réussi à obtenir, et à fixer plusieurs formes intermédiaires entre les deux Bacillus, non seulement par leurs propriétés physiologiques, mais aussi par leurs caractères morphologiques. La transformation la plus rapide a été réalisée à la surface d'une solution d'extrait de viande (1 p. 100) et de sucre (3 p. 100) additionné de jaune d'œuf. M. Pasteur a annoncé récemment qu'il n'avait pas obtenu trace d'une pareille transformation du $B$. anthracis, en bactérie du foin, malgré 130 cultures dans l'humeur aqueuse de l'œil (Revue scient., 16 sept. 1882).

D'après M. Zopf, les genres Cladothrix, Beggiatoa, Crenothrix, offrent urie très grande variété de formes dans leur développement; 
leurs états négatifs se présentent successivement sous l'apparence de Micrococcus, Monas, Leptothrix, bàtonnets et spirales; la bactérie ne serait donc pas une forme indépendante, mais un des états d'un cycle complexe. Toutes les bactéries ne posséderaient cependant pas un pareil polymorphisme, et quelques-unes n'affectent qu'une seule forme (Bacillus subtilis, B. anthracis). Dans chaque état elles peuvent être mobiles ou immobiles. M. Zopf dit avoir observé directement les trans. formations indiquées.

\section{SACCHAROMYCE்TES.}

Les Saccharomyces diffèrent des bactéries par leur mode de reproduction; ils se multiplient, en effet, par bourgeonnement. Ils possèdent des affinités d'un côté avec les bactéries, de l'autre avec les cham. pignons vrais, vers lesquels ils pourront encore servir de transition. Les Saccharonyces sont des protophytes unicellulaires, ovales, fusiformes ou elliptiques, à membrane mince, à contenu finement granuleux, présentant des gouttelettes graisseuses et des vacuoles; ils sont dépourvus de noyau.

Pour la reproduction, un refoulement se fait, à une des extrémités de la cellule, de dedans en dehors, donnant naissance à un petit bourgeon dans lequel s'accumule le protoplasma. Le bourgeon augmente de volume, tout en restant en communication avec la cellule mère par un orifice plus ou moins étroit; de sphérique il devient fusiforme; puis une cloison le sépare de la cellule-mère, de laquelle il se détache tôt ou tard, pour vivre d'une façon indépendante et bourgeonner à son tour. Ordinairement avant que ce premier bourgeon soit aussi avancé, il en apparaît d'autres sur la cellule-mère, soit à côté du premier, soit à l'extrémité opposée. Quand les générations, issues les unes des autres, restent plus ou moins longtemps en connexion, il en résulte des chapelets, ou plutôt des colonies ramifiées. La cellule-mère, après avoir produit plusieurs générations de filles, finit par s'appauvrir et vieillir ; finalement, elle se flétrit et meurt. 
Dans le Carpozyma apiculatum (M. Engel) ou Saccharomyces apiculatus, le bourgeonnement est un peu différent. Les bourgeons se forment uniquement sur les deux saillies qui caractérisent cette espèce ; ils apparaissent l'un après l'autre et forment d'abord avec la cellulemère, une file longitudinale de trois éléments; arrivés au terme de leur croissance, ils se replient à leur point d'insertion et deviennent perpendiculaires à la cellule-mère qu'ils flanquent ordinairement à droite et à gauche.

Le Saccharomyces mycoderma (Mycoderma vini) forme des colonies abondamment ramifiées; d'après $\mathbf{M}$. Cienkowski, il donne aussi un mycelium, quand l'oxygène commence à faire défaut; les cellules de ce mycelium ne tardent pas à se séparer les unes des autres.

Quand l'oxygène est en abondance, l'épuisement du liquide nutritif détermine la formation de spores; elles sont beaucoup plus rares dans la levûre de bière cultivée, Saccharomyces cerevisice, que dans les autres espèces. Tout le protoplasme d'une cellule agrandie se divise, dans l'espace de vingt-quatre heures, en deux à quatre balles qui s'entourent chacune d'une épaisse membrane (M. Rees). Dans le Carpozyma (M. Engel) on voit un petit amas de matière protoplasmique, clair et brillant, se former à l'une des extrémités de la cellule, du côté de la saillie; il s'agrandit sans changer de place, puis chemine vers le centre, en entraînant après lui un prolongement effilé; là il devient sphérique et plus gros. Quelquefois, un pareil amas se forme à chacune des extrémités; mais, pius tard, ils se rejoignent et se confondent au centre. La membrane cellulaire s'épaissit et finit par présenter deux contours nets, séparés par un espace clair et rosé, les apicules disparaissent. La sphère centrale s'entoure d'une membrane d'abord mince, puis épaisse et différenciée en trois couches; elle passe. ainsi l'hiver. Au printemps suivant, elle s'accroît de nouveau, rompt son enveloppe externe et se transforme en sporange; dans son intérieur on voit, en effet, apparaître de petits amas granuleux, ébauches des spores futures. Dans le Mycoderma vini les spores sont au nombre de une à quatre par cellule (M. de Seynes).

Tandis que les cellules ordinaires desséchées périssent au bout de deux à quatre semaines, les spores conservent plusieurs mois leur 
faculté germinative: placées dans des conditions favorables, elles semblent se développer comme les cellules ordinaires, après destruction de la membrane de la cellule-mère.

Le Saccharomyces cerevisice, le mieux connu de tout ce groupe, bourgeonne entre 8 et $35^{\circ} \mathrm{C}$.; l'optimum de température varie avec les différentes races, levûre haute, levûre basse, qu'il est, d'ailleurs, possible de transformer les unes dans les autres par des cultures succes. sives. Toute multiplication s'arrête à $+3^{\circ}$ : mais les cellules résistent à des froids intenses, jusqu'à $-113^{\circ}$. Dans l'eau, la levûre meurt à $75^{\circ}$; sèche, elle peut être chauffée jusqu'à $100^{\circ}$.

Les spores des Saccharomyces sont très répandues; on en trouve à la surface de tous les fruits sucrés, et ils provoquent ainsi la fermentation du vin. D'après M. Brefeld, les Saccharomyces déposés sur les feuilles et les fruits, se multiplieraient abondamment dans l'intestin, puis dans les fèces des animaux herbivores et seraient ensuite dispersés par le vent. M. Boutroux pense que les levûres en voie de développement, en été, sur les fruits, passent l'hiver (à l'état de spores) sur les débris de ces fruits, ou dans les habitations des insectes ou dans la terre où elles ont été entraînées par la pluie; au printemps, les germes conservés sont portés de nouveau par les insectes sur les fleurs nectarifères, où ils se multiplient et s'ensemencent de fleur en fleur; finalement, les insectes les transportent encore sur les fruits mûrs qu'ils vont visiter un peu plus tard. M. Hansen, de son côté, a constaté que le Carpozyma apiculatum est transporté par la pluie, des fruits dans la terre où il se conserve durant l'hiver; la terre recueillie sous les arbres fruitiers permet, en effet, d'obtenir une riche yégétation de cette levûre.

Plusieurs fermentations sont corrélatives de la vie et du développement des Saccharomyces. Le $S$, vini brûle l'alcool du vin et le transforme en eau et acide carbonique. La fermentation alcoolique est provoquée, non seulement par tous les Saccharomyces, mais aussi par divers Mucor, Aspergillus, Penicillium, c'est-à-dire par de véritables champignons; cette circonstance n'a pas pcu contribué à faire croire à un polymorphisme qui, en réalité, n'existe pas.

Les cellules de ces différents végétaux, dans ies circonstances ordi- 
naires, en présence de l'oxygène, consomment du glucose pour leur accroissement et leưr multiplication; elles le brûlent partiellement en le transformant en eau et acide carbonique; on a supposé que, dans ce cas, le glucose se change d'abord en acide carbonique et alcool, (première transformation); l'ulcool, par oxydation, serait changé, par exemple, en eau et acide acétique, dont la molécule, se conden* sant immédiatement, donnerait de nouveau du glucose (deuxième transformation). Quand on plonge les cellules dans la solution sucrée, celle-ci fermente, c'est-à-dire donne de l'eau et de l'acide carbonique; dans ces circonstances nouvelles, la première transformation continue activement, mais la deuxième se trouve considérablement ralentie, faute d'oxygène; l'alcool et l'acide carbonique, en grand excès dans les cellules, passent dans la solution. Les cellules continuent, néanmoins, à croître et à se multiplier en consommant l'oxygène dissous dans le liquide ou faiblement combiné, ou mis en réserve par la cellule elle-même; une fois cet oxygène épuisé, la multiplication s'arrête; mais les cellules déjà formées ne cessent pas pour cela de vivié et d'opérer la première transformation, grâce à leur résistance à l'asphyxie. Cette résistance est très variable suivant les diverses plantes énumérées plus haut; c'est pour cela que celles-ci sont des ferments alcouliques plus ou moins parfaits. A ce point de vue, les Saccharomyces sont caractérisés par leur très grande résistance à l'asphyxie; c'est pour cela aussi que les brasseurs ont l'habitude très rationnelle de laisser la levûre au contact de l'air; elle fait ainsi la pro. vision d'oxygène ināispensable à son développement et à la bonne marche de la fermentation.

La végétation peut être accompagnée de la production d'une diastase inversive di sucre de can.e (Saccharomyces) et alors le ferment peut opérer aussi la transformation en alcool du sucre de canne; quand cette diastase fait défaut, cela a lieu pour les Mucor spinosus, $M$. circinelloides par exemple (M. Gayon), le ferment ne peut s'attaquer qu'au glucose.

Du reste, toutes les cellules végétales renfermant du glucose, sont susceptibles de produire de l'alcool, quand on les prive d'oxygène; la production de l'alcool est, en effet, une conséquence de l'asphyxie de 
la cellule; seulement, toutes les cellules ne sont pas susceptibles de se multiplier, quand l'oxygène n'existe plus qu'en quantité très minime (libre ou faiblement combiné), ni susceptibles de s'accroître, l'oxygène faisant défaut, et c'est en cette différence que consiste la caractéristique des ferments (M. Pasteur).

\section{SCHIZOPHYCÉES}

Les Schizophycées, appelées aussi Cyanophycées ou Oscillariées, montrent une étroite parenté avec les Schizomycètes; aussi, les a-t-on réunies quelquefois en un seul groupe, celui des Schizophytes (M. Cohn). Quoique possédant de la chlorophylle, les Schizophycées ne se distinguent même pas physiologiquement, d'une façon absolue, des Bactéries; elles vivent, en effet, souvent dans le tissu de plantes élevées, ou dans des milieux, tels que : eaux stagnantes, vase marine, où aucune autre algue ne pourrait exister. Elles ont encore de commun, avec le grompe précédent, la résistance qu'elles présentent, en partie au moins, à de hautes températures; certaines Oscillariées vivent, en effet, dans les eaux thermales au-dessus de $50^{\circ}$; ou même dans les solfatares. Elles peuvent également, dans certaines circonstances favorables, se multiplier avec la même prodigieuse rapidité et possèdent enfin fréquemment de prétendus cils, tout comme les Bactéries; les noyaux manquent. Les Leptothrix, les Beggiatoa sont, en réalité des Oscillariées sans chlorophylle; d'un autre côté, celle-ci existe dans de véritables Schizomycètes : les Bacterium viride, le Bacillus virens. Un caractère différentiel, plus général, réside dans les spores endogènes des Bactéries; tandis que les Oscillariées n'ont que des cellules durables; mais ici même, le Leuconostoc servirait d'intermédiaire (M. Van Tieghem), Un grand nombre de Schizophycées se rencontre dans le thalle des Lichens.

Le nom de Cyanophycées leur est souvent donné, parce qu'elles renferment toujours, à côté de la chlorophylle, une matière colorante 
bleu-verdattre, la phycocyanine ; elle n'est, ordinairement, pas seule et ces algues, comme les Bactéries, présentent des couleur's très variées; elles ont aussi une grande tendance ì gélifier leurs membranes et à vivre en colonies.

Des zoospores ont été oiservées dans une Chroococcacée, Mcrismopoedia (M. Borzi); exi dehors de ce cas isolé, au moins jusqu'à présent, les Schizophycées nese reproduisent, comme les Schizomycètes, que par scissiparité et par spores ou plus exactement par cellules durables (MM. Bornet, Thuret, de Bary, Nageli, Fischer, Zanczewski, etc.)

Scissiparité. - Les Chroococcacées, qui ne se distinguent quelquefois des Palmellacées que par leur phycocyanine, sont unicellulaires et se segmentent alternativement dans deux ou trois directions perpendiculaires, quelquefois dans une seule direction, mais sans former de filaments, comme dans les familles suivantes. Les cellules isolées sont indépendantes; elles peuvent cependant rester englobées dans une substance gélatineuse, provenant des enveloppes des cellulesmères; dans les Glcoocapsa ces enveloppes, plus résistantes, restent emboîtées les unes dans les autres. Dans les Oscillariées les cellules ont la forme de disques et sont réunies en filaments cylindriques, mobiles, plus ou moins contournés, enveloppés d'une substance gélatineuse; la segmentation se fait toujours perpendiculairement à la longueur du.filament. Il en est de même dans les Nostochinées; mais leurs filaments présentent, de distance en distance, des cellules-limites ou hétérocystes qui ne se multiplient pas, possèdent une membrane plus épaisse et perdent leur plasma. A certains moments, l'enveloppe gélatineuse devient diffluente, et les hormogonics, comprises entre les hétérocystes, acquérant la mobilité, se déplacent en rampant et vont se fixer, de nouveau, plus loin; elles s'eutourent d'une gaîne membraneuse. Leurs cellules alors se segmentent une ou deux fois parallèlement à l'axe longitudinal du filameni; les petites rangées transversales résultantes se soudent bout à bout, de façon à reconstituer un filament unique en zigzag dans lequel apparaissent de nouveau des hétérocystes (Thuret). Dans le Nostoc paludosum (M. Janczewski) 
le processus est différent; l'hormogonie droite se fixe par ses deux extrémités; les cellules intermédiaires se divisent transversalement, et le filament devient ainsi de plus en plus ondulé; les hẹ́térocystes èt la gelée apparaissent enșuite.

Les Rivulariacées sont plus différenciées; chaque filament est terminé d'un côté par une cellule basilaire (hétérocyste), de l'autre, par un poil hyalin articulé; les cellules intermédiaires, seules, se segmentent transversalement; au milieu d'elles apparaît une nouvelle cellule basilaire; la portion de l'ancien filament, placée au-dessous d'elle, se déplace un peu latéralement et reforme un nouveau poil à sa partie supérieure; puis, les deux nouveaux filaments résultants se juxtaposent. Les cellules actives, seules, prennent part à la formation des hormogonies; pour cela elles se multiplient; quelques-unes se changent en hétérocystes, bientòt comprimés en disques par les cellules qui restent actives et qui résorbent les párois transversales de ces disques, de sorte que chaque hétérocyste se trouve réduit à un anneau. Les hormogonies émigrent de la gaîne, entraînant ces anneaux ; puis chacune donne un nouveau filament.

Les filaments des Scytonemées s'accroissent à leurs deux extrémités au moyen de grandes cellules terminales, dans le voisinage desquelles la gaîne gélatineuse s'amincit beaucoup; au milieu de chaque rangée apparaissent des hétérocystes qui la divisent en fragments. Chaque fragment, restant en place, continue à se multiplier à ses deux extrémités; mais, au point de rencontre de deux fragments, les cellules nouvelles ne trouvant pas de place, forment des rangées perpendiculaires au filament ancien et ordinairement geminées. Le tout simule alors un filament ramifié; mais, en réalité, les fragments sont des filaments distincts que la gélatine seule tient réunis ensemble; ils peuvent, du reste, s'isoler ultérieurement. De plus, il y a encore reproduction par hormogonies.

Les Sirosiphonées (Stigonemées) se multiplient encor'e par leurs cellules terminales; les filaments deviennent pluriseriés par segmentation longitudinale des cellules du filament, dont quelques-unes donnent, en outre, des branches perpendiculaires au premier axe. Les hormognies ne se forment que dans ces branches. 
Spores. - Pour la formation des spores, toutes les cellules d'une colonie de Chroococcacées s'entourent, en même temps, chacune d'une membrane épaisse; chaque cellule durable reproduit une famille à la germination (Gloeocapsa). Les filaments des Oscillariécs supportent la gelée et la dessication; ils restent donc simplement au repos pendant l'hiver; quelquefois les filaments entiers s'enkystent comme le font ailleurs les cellules isolées (M. Borzi).

Quand les Nostocs sont menacées de dessication ou de gelée, certaines de leurs cellules végétatives se dilatent, s'allongent, se gorgent de gouttelettes huileuses et épaississent leurs membranes (M. Janczewski); ce sont alors des spores durables. Elles peuvent prendre naissance en petit nombre dans un filament (Spermosira), envahir le filament entier (Nostoc), ou se restreindre à une cellule unique à côté des hétérocystes (Cylindro-spermum, Sphaerozyga); dans ce dernier cas, elles prennent une forme cylindrique ou ellipsoïde. Leur membrane est souvent couverte de proéminences ou de piquants; elle est plus ou moins colorée, et se déchire à la germination. Le germe se segmente quelquefois déjà dans l'intérieur de la spore (Spermosira); puis il s'allonge et forme un filament d'un nombre variable de cellules; aux extrémités du filament apparaissent des hétérocystes.

Dans les Rivulariacées (M. de Bary) la cellule végétative attenante à la basilaire, se transforme en un large cylindre (manubrium), a protoplasma dense, à membrane dure et solide: c'est une pore qui passe seule l'hiver. A la germination, elle se divise en cellules par des cloisons transversales et donne alors directement un ou plusieurs filameuts, ou encore plusieurs hormogonies. Dans les Scytonemées, les cellules cylindriques se transforment en spores sphériques qui restent réunies en amas réguliers; il en est de même dans les Sirosiphonées.

D'après M. Zopf, il y aurait encore ici polymorphisme; des filaments de Nostochinées, se transformeraient en colonies (zooglæa) de Chroococcacées. 


\section{LES ALGUES}

Les Cyanophycées font déjà en réalité partie des Algues, et au point de vue de la reproduction, comme du corps végétatif, ce sont en effet les Algues les plus simples. Le protoplasma vert peut cependant exister sous une forme plus simple encore; M. Van Tieghem a deirit un petit être, le Dimystax Perrieri, qui semble plus rapproché du point de départ des Algues; c'est une masse gélatineuse tremblotante, rendue verte par la chlorophylle; elle possède une touffe de cils vibratiles, d'où partent deux bandes plasmiques également ciliées; ces cils s'agitent, mais sans amener le déplacement du corps; celui-ci, à un moment, se divise, par segmentations successives, en 16 cellules dont chacune devient à peu près semblable à la mère. Quoique le protoplasma soit vert, il ne sécrète pas de cellulose; c'est là une exception : les autres Algues s'entourent d'une membrane au moins pendant une partie de leur existence. Dans un groupe restreint d'Algues vertes, le protoplasme n'est même jamais nu, ce sont les Conjuguées; ce caractère et l'absence de mouvements libres les rapprochent des cyanophycées. D'autres, au contraire, se meuvent toute leurvie; quoique munies d'une membrane de cellulose, elles conservent 
donc un certain caractire d'animalité; ce sont les Volvocinées. Ces deux petits rameaux spéciaux diffèrent assez notablement de la grande branche des Algues vertes; le protoplasma de celles-ci est habituellement immobile et renfermé dans une membrane, mais ces plantes passent toujours dans le cycle de leur développement par une phase pélagique. Ce sont précisément les organes reproducteurs qui possèdent ce caractère de motilité; il se conserve le plus longtemps dans les éléments mâles, qui sont encore pélagiques, dans les deux grands groupes de Cryptogames terrestres.

Les Algues offrent des modes de reproduction très variés. Les zoospores sont les agents les plus ordinaires de la multiplication asexuée : ce sont de petites cellules nues et mobiles qui naissent par contractions et partitions répétées du contenu d'une cellule, quelquefois aussi sans division, la cellule toute entière ne formant qu'une seule zooopore (Edogoniées). Elles s'allongent antérieurement en une pointe hyaline le rostre, portant deux cils (Algues vertes); rarement le nombre des cils est de un (Botrydium) ou de quatre (Ulva), etc. D'autres fois, ces cils sont implantés latéralement (Fucoüdées). Les zoospores semblent munies d'un noyau qui a été constaté d'une façon certaine, au moins dans quelques cas ; elles possèdent en outre ordinairement une tache rouge et des vésicules pulsatiles. Les zoospores sont colorées en vert, dans les Chlorophycées, et en brun dans les Fucoidées. Dans les Floridées, les cellules reproductives asexuées deviennent immobiles, de même que les anthérozoïdes, ce qui contribue encore à donner à la reproduction de ce groupe un caractère spécial.

La reproduction sexuée se présente déjà dans certaines Algues avec tous ses caractères essentiels; elle les conservera dans les Muscinées et les Cryptogames vasculaires dont les organes sexuels acquièrent seulement une différenciation plus grande. Elle consiste dans la fusion entre deux cellules, l'anthérozoïde et l'oosphère, produits dans des organes différents, et formant, par leur réunion, l'œuf; celui-ci se développe immédiatement ou après un temps de repos en une génération nouvelle. Dans ce second cas, l'œuf s'entoure d'une membrane épaisse et résistante; il peut alors supporter le froid et la dessiccation; 
aussi est-ce seulement, dans beaucoup d'Algues, quand la plante est menacée par de pareilles causes de destruction, que la fécondation a lieu. La reproduction sexuée n'apparait donc pas encore dans la majorité du groupe et d'une façon régulière, mais seulement comme un acte. spécial adapté à certaines circonstances, et une génération sexuée ne survient qu'après une longue série de générations asexuées; l'œuf, en germant, donne naissance de nouveau à un individu asexué, soit directement, soit par i'intermédiaire de zoospores. Cependant, dans certaines Algues (Fucacées) les générations sexuées se suivent sans interruption, et on ne connaît même pas d'individus asexués.

Les anthérozoïdes, ou éléments mâles, sont produits dans des organes particuliers : les anthéridies. Ils offrent la plus grande ressemblance avec les zoospores, sont mobiles comme celles-ci et représentent de véritables cellules. Quand l'anthérozoïde rencuntre l'oosphère, les deux cellules se fondent, noyau avec noyau, et protoplasma avec protoplasma; puis l'oospore qui résulte de cette copulation s'entoure aussitôt d'une membrane. Dans la fécondation typique, l'oosphère reste immobile; la cellule qui l'a produite ou l'oogone s'ouvre au moment de la maturité, ce qui ouvre l'accès aux anthérozoïdes; souvent la fusion peut avoir lieu par un point quelconque de l'oosphère (Fucus), d'autres fois la place est indiquée d'avance par une region plus claire, la tache réceptrice. Il est évideat qu'une pareille copulation n'est possible que par l'jntermédiaire de l'eav.

Le caractère de la fécondation est tout autre dans certains groupes spéciaux (Floridées, Conjuguées), comme nous le verrons plus loin. Dans beaucoup dAlgues vertes et brunes, la fécondation sexuée a lieu d'après le mode indiqué plus haut. Il ne semble y avoir tout d'abord aucune analogie entive cette reproduction et celle par zoopores; en réalité, il existe de très nombreux intermédiaires. Déjà, dans les Myomycetes, nous avons vu une fusion indifférente de deux ou plusieurs cellules mobiles. Des copulations entre zoospores se rencontrent aussi dans les Algues; il est vrai qu'elles deviennent alors sexuées et perdent isolément leur faculté germinatrice; dans quelques cas il n'y a aucune différence entre les deux cellules qui se réunissent; d'autres 
fois, l'une devient plus grande, ou la copulation n'a lieu qu'après que l'une des deux cellules égales est arrivée au repos; ces deux modifications se trouvent réunies dans les Cutleria, et il suffit alors que la grande zoospore, qui devient immobile, ait une vie pélagique de plus en plus réduite, pour que finalenıent elle reste à l'état d'oosphère dans la cellule où elle a pris naissance. Quelquefois les zoospores sexuées égales se développent cependant isolément (Ulothrix), ce qui affaiblit encore la distinction absolue entre les deux espèces de reproduction; la grande zoospore sexuée peut aussi germer sans fusion avec l'élément mêlé (Ectocarpus). Ce phénomène, appelé parthénogenèse, se rencontre plus fréquemment dans les plantes à oosphères immobiles (Ocdogonium, Cylindrocapsa, Bulbochaete); il devient la règle chez la Chara crinita.

A.près les Conjuguées et les Volvocinées nous passerons en revue la grande série des Algues vertes ou Chlorophycées, composée des Protococcacécs, Siphonées et Confervacées; les représentants les plus élevés de cette branche conduisent aux Muscinées. Les Chara peuvent être rattachés à la même série; c'est en réalité un groupe quelque peu aberrant dont la place n'est pas encore bien déterminée. Les Fucoïdées et les Floridées sont deux branches latérales d'Algues, distinctes de la précédente par d'assez nombreux caractères. Les premières, dites aussi Mélanophycées, sont plus voisines des Chlorophycées; on ne les trouve que dans la mer; c'est surtout ce groupe qui nous offrira des transitions intéressantes entre la fécondation typique et la copulation des zoospores. I.es Floridées, marines aussi en grande majorité, occupent une place à par par leur mode de fécondation et par l'immobilité de leurs spores et anthérozoïdes, c'est par elles que nous terminerons les Algues.

\section{CONJUGUÉES.}

Dans les Conjuguées le protoplasma n'est jamais nu; il reste constamment enfermé dans la membrane cellulaire, mème pour la 
fécondation, et cela les distingue de la grande majorité des Algues. On ne les trouve que dans l'eau doure.

Les Conjuguées constituent une série particulière partant, soit des protistes, soit plutôt de la grande série des Algues vertes; c'est un groupe homogène, mais restreint, qui ne semble avoir été susceptible que d'un perfectionnement très limité.

La reproduction asexuée consiste partout en une simple division cellulaire ; quand il s'est formé deux noyaux, par la partition du noyau primitif, Ia cloison cellulaire prend naissance à son tour, en direction centrip̣ète; les cellules ainsi constituées restent ordinairement réunies entre elles, de façon à former des filaments cylindriques qui se rompent en fragments, quand ils ont atteint une certaine longueur, et qui peuvent être considérés plutôt comme des colonies que comme des individus. En effet, dans la famille des Desmidiacées, les cellules sont tantôt réunies en filaments et tantôt isolées; elles se composent chacune de deux moitiés symétriques, de deux cônes, par exemple (Closterium), réunis par leur base; mais leur forme peut être aussi celle d'une hémisphère et alors il existe un étranglement profond (Cosmarium) au point de soudure de ces deux moitiés qui contiennent chacune deux grains d'amidon et huit plaques de chlorophylle. La division se fait toujours dans la portion étranglée moyenne de la cellule; une déchirure annulaire se produit là dans la membrane résistante externe; la membrane interne mince s'allonge ensuite, écartant les deux moitiés symétriques de la cellule, en constituant un isthme qui les relie; dans cet isthme apparaît la cloison de séparation qui ne tarde pas à se fendre en deux lamelles. Chacune des cellules filles est composée alors de deux parties fort inégales; la partie la plus petite et la plus jeune n'est entourée que d'une mince enveloppe de cellulose; mais elle grandit rapidement de façon à égaler son aînée; puis sa membrane prend aussi plus de consistance. En même temps, les plaques chlorophylliennes de la moitié ancienne s'allongent dans la nouvelle; ses deux grains d'amidon se divisent pour en former quatre dont deux aussi émigrent dans la moitié nouvelle et ainsi se constituent deux individus semblables entièrement à la cellulèmère. 
La reproduction asexuée se confond donc avec la division cellulaire. Quant à la fécondation, elle présente une certaine différenciation progressive, mais jamais le protoplasma ne se meut autrement que par reptation, pendant l'acte sexuel.

Dans le genre Gonatonema, il ne semble y àvoir encore qu'une tendance à la fécondation qui jamais ne s'effectue (M. Wittrock); il se forme seulement des pseudozygospores.

Dans le genre Mougeotia, qui constitue avec le précédent la famille des Mesocarpées, deux filaments se placent parallèlement l'un à l'autre, et la membrane de chaque cellule forme une protubérance latérale qui se rencontre avec la protubérance correspondante de la cellule d'en face; au point de contact il y a soudure, puis résorption de la paroi commune, ce qui établit une communication entre les deux cellules en regard. Les corps protoplasmiques, sans se contracter, suivent ces protubérances et se fusionnent au milieu du canai de communication, sauf une faible couche plasmique qui reste adhérente à la paroi des deux cellules et qui leur permet de manifester encore une certaine vitalité après la constitution de la zygospore, en prenant part, avec celle-ci, à la formation des cloisons. Ces cloisons séparent le canal de communication des deux cellules reproductives; il peut n'y en avoir qu'une de chaque côté, de sorte que, finalement, il y aura trois cellules. Mais quand la zygospore, plus grande, se prolonge dans les cellules primitives, il faudra deux cloisons de séparation dans chacune de celles-ci, une en haut et une en bas, de sorte que finalement il se sera formé cinq cellules. Par un procédé intermédiaire entre les deux précédents, il pourra s'en former quatre. Comme ces trois cas ont été observés dans une même paire de filaments (M. Wittrock), il n'y a pas lieu de diviser, d'après cette différence, le genre Mougeotia en plusieurs autres.

La conjugaison est donc complètement égale, au moins pour nos moyens actuels d'investigation, et les deux masses plasmiques font autant de chemin l'une que l'autre. Il en est de même dans les Desmidiacées; la seule différence c'est qu'ici le protoplasma, tout entier, est employé à la copulation, à la suite de laquelle les cellules copulantes meurent immédiatement. Ces cellules sont toujours, au moment 
de la fécondation, isolées de leur colonie; elles peuvent disposer leurs axes parallèlement(Closterium) ou perpendiculairement (Cosmarium). La conjugaison semble avoir lieu de préférence entre cellules sœurs. Les protubérances prennent naissance sur l'isthme, reliant les deux moitiés symétriques de chaque cellule; leur formation est précédée d'une déchirure de la membrane externe résistante ; le canal de communication qui relie les deux isthmes peut s'isoler (Сosmarium) ou ne pas s'isoler (Closterium) par des cloisons des deux cellules en copulation.

Les Zygnémacées diffèrent des deux familles précédentes en ce que, après la formation du canal de communication et avant la conjugaison, le protoplasma total de la cellule se contracte toujours et se rassemble en une masse elliptique autour du noyau et au milieu de la cellule, dans les Zygonium chacune de ces masses fait encore la moitié du chemin ; mais dans les Zygnema et les Spirogyra une des deux masses reste immobile, c'est l'oosphère; l'autre, l'anthérozoïde, se déplace seule; ordinairement même tout un filament est mâle et l'autre femelle. Cependant la différenciation sexuelle semble ici bien peu ac-centuée, puisqu'il arrive quelquefois que la copulation ait lieu entre deux fragments d'un même filament. Dans les Spirogyra même, deux cellules adjacentes peuvent ainsi fusionner leur contenu par un canal de communication parallèle alors au filament.

Un pas de plus se trouve fait chez lies Sirogonium; quand la communication est établie, la cellule femelle se divise en deux cellules filles; l'une petite, végétative, l'autre grande et sexuelle en rapport. avec le canal. La cellule mâle se divise à son tour en une grande cellule végétative et une petite sexuelle ou en deux cellules végétatives et une sexuelle placée au milieu. Puis seulement le protoplasma se contracte dans les deux cellules devant conjuguer et l'anthérozoïde se glisse auprès de l'oosphère toujours plus grande que lui.

On voit donc qu'il existe chez les Conjuguées une différenciation sexuelle progressive, mais qu'ellen'est jamais bien accentuée. Ce qui le prouve, c'est que trois cellules peuvent conjuguer ensemble, et qu'une cellule unique, n'ayant point rencontré de congénère, n'en forme pas moins une spore, dite alors azygospore ou pseudo-zygospore, qui se comporte comme les vraies zygospores. 
Dans les cas précités, la zygospore se forme par la fusion des deux noyaux en un seul et par la réunion des deux masses protoplasmiques avec contraction; la zygospore s'entoure ensuite d'une membrane propre dans laquelle on distingue plus tard trois couches; elle devient libre par destruction des filaments vides et elle passe ainsi l'hiver; au printemps les couches externes sont rompues; le protoplasma entouré de la mince membrane interne s'allongre, constituant une cellule qui ne tarde pas à se diviser en deux. Dans les Desmidiacées ces deux cellules se comportent exactement de la même façon et régénèrent bientôt une seconde moitié semblable à la première, sauf la membrane résistante qui manquera toujours aux deux plus anciennes moitiés. Dans les deux autres familles au contraire, une des deux premières cellules reste indivise et conserve une forme particulière, l'autre seule se multiplie par bipartitions; cette différence fonctionnelle des deux premières cellules serait une raison pour considérer les filaments entiers comme des individus et non comme des colonies.

\section{DIATOMÉES.}

Les Diatomées ou Bacillariacées se distinguent des Algues vertes par la présence d'un principe colorant brun, diatomine ou phycuxanthine ; mais ieur mode de reproduction agame aussi bien que sexuée, les rapproche des Conjuguées et surtout des Desmidiées, de plus, comme ces dernières, elles peuvent vivre en colonies ou s'isoler; ce qui les caractérise, c'est la présence de la silice dans leur paroi cellulaire.

1 Chaque diatornée est renfermée dans une membrane solide silicifiée, composée de deux valves; chaque valve est formée d'un fond et d'un rebord. L'une des deux valves recouvre l'autre par son rebord; le tout représente une boîte avec son couvercle. Pour la reproduction asexuée, identique ici encore avec la division cellulaire, les deux valves s'écartent l'une de l'autre par glissement de leurs rebords. Au 
moment où ces bords vont se séparer, une cloison transversale apparaît entre les deux moitiés; elle se divise bientôt en deux lamelles. Chaque lamelle n'aura plus qu'à se transformer en une valve nouvelle emboîtée dans l'ancienne pour qu'il y ait deux individus nouveaux. Le même processus se répétant pour toutes les cellules filles, il est évident que les descendants d'une même cellule mère seront de plus en plus petits, au moins en majorité. Cette décroissance a une limite, quand elle est atteinte les valves s'écartent comme pour une bipartition, mais le protoplasma s'échappe cette fois entre les deux valves, s'allonge notablement (auxospore), et s'entoure d'une membrane non silicifiée, à l'intérieur de laquelle deux valves, plus grandes que l'ancienne, prennent naissance. Que deux individus se juxtaposent pour former chacun une ou quelquefois deux auxospores, que deux auxospores d'origine dfférente se fusionnent en une seule, et nous avons la génération sexuée qui ne s'observe cependant que rarement; quelquefois aussi les auxospores viennent en contact sans se fusionner.

La reproduction est donc tout à fait analogue à celle des Desmidiacées.

\section{VOLVOCINÉES.}

Les Volvocinées constituent un petit groupe d'êtres conservant un certain caractère d'animalité, puisqu'ils se meuvent constamment ì l'aide de cils, mais intimement liés néanmoins, par leur enveloppe de cellulose et leur chlorophylle, aux algues vertes.

L'état de zoospore, qui n'est que transitoire dans les autres Algues, persiste ici pendant toute la vie de l'individu.

Les Volvocinées consistent en cellules munies de deux cils ; ceux-ci sont implantés sur un rostre coloré en vert, comme tout le protoplasma, par de la chlorophylle non granuleuse; souvent une tache rouge et deux vésicules pulsatiles existent encore dans le voisinage du rostre. La membrane cellulaire adhère étroitement au corps protoplasmique dans la région des cils qui la traversent. Chez les Chla- 
mydomonas où les individus vivent isolés, elle s'écarte postérieurement de la zoospore, laissant un espace rempli de suc cellulaire ou de mucilage. Les individus des autres genres vivent réunis en famille; dans les Gonium, 4, 6 ou 8 cellules rayonnantes forment un disque avec cils à la circonférence; ici la membrane est commune et au centre du disque existe une cavité cylindrique. Les Stephanosphaera présentent une disposition semblable avec une membrane renflée en sphère. Dans les Pandorina la sphère est entièrement remplie par les 16 individus qui se touchent au centre. Dans les Eudorina les 16 à 32 cellules plus espacées laissent une cavité centrale, et les Volvox représentent des Eudorina dont la sphère peut contenir jusqu'à 12,000 individus.

Pour la multiplication asexuée chaque individu se divise en autant de cellules qu'il y avait de membres dans la famille; ces cellules se disposent comme dans la famille mère, secrètent en même temps une membrane commune, acquièrent des cils, et les jeunes familles ainsi formées se meuvent alors dans la membrane de la-cellule mère; par résorption de celle-ci elles ne tardent pas à devenir libres. Ce n'est qu'après achèvement des divisions que les nouvelles cellules, jusqu'alors de plus en plus petites, commencent à s'agrandir.

Dans les Chlamydomonas la famille n'est composée que d'un seul individu, et chacune des cellules filles, au nombre de deux à quatre, s'entoure d'une membrane avant de s'échapper de l'enveloppe de la cellule mère. Dans les Eudorina et Volvox les jeunes cellules sont d'abord disposées côte à côte en disque; mais à mesure que leur nombre augmente, ce disque s'infléchit par ses bords qui finissent par se rejoindre, de telle façon que la lamelle s'est transformée en sphère creuse ; en même temps la division cellulaire s'est achevée. De plus, dans les Volvox, ce ne sont pas toutes les cellules d'une famille, mais quatre seulement ( $V$. minor) ou huit (Volvox globator), disposées régulièrement, qui se segmentent et qui donnent de nouvelles familles; tous les individus de la colonie ne sont donc déjà plus identiques.

La génération sexuée est plus variée.

Le Chlamydomonas multifilis, les Gonium, Stephanosphaera et Pandorina forment tous des zoospores nues, sans membrane, qui 
semblent sexuées (planogamètes), et qui ne peuvent germer suns doute qu'après copulation ; cela est prouvé du moins pour la première espéce et pour les Pandorina qui serviront d'exemple. Après une série de générations asexuées, prend naissance une famille sexuée (à l'approche de l'hiver ou sous menace de dessiccation) qui ne se distingue des précédentes que parce que ses membres se décomposent chacun en un nombre moindre de cellules, ordinairement 8 au lieu de 16. Ces cellules deviennent libres sous forme de zoospores nues, de taille très inégale, ce qui ici n'influe en rien sur la sexualité; car chaque zoospore copule avec une autre, égale de grandeur ou inégale, peu importe; il semble seulement que la conjugaison ne peut avoir lieu entre les membres d'une même famille. Les deux corps protoplasmiques se confondent et l'oospore (zygote) qui en résulte possède d'abord deux taches rouges et quatre cils; mais bientôt elle perd ceux-ci, et s'entoure d'une membrane épaisse.

Chez les Eudorina et Volvox, il y a une véritable fécondation sexuelle dans une famille spéciale qui ne produit point de familles filles.

Dans les Eudorina certains membres de la colonie se transforment en oosphères peu différentes des autres cellules, et restant comme elles englobées dans la famille; d'autres membres deviennent des anthéridies. Celles-ci se divisent en nombreux anthérozoïdes par une segmentation entièrement analogue à celle des cellules végétatives, sauf que le disque ne se recourbe pas en sphère. Les anthérozoïdes, munis de deux cils à la pointe du rostre, s'échappent, puis pénètrent du dehors dans les oosphères par la région des cils; l'oospore ainsi formée s'entoure bientôt d'une membrane.

Dans les Volvox les oosphères, volumineuses et proéminentes dans l'intérieur de la sphère, sont au nombre de huit ordinairement dans le Volvox minor, tandis que le Volvox globator en présente jusqu'à quarante; elles proviennent d'autant de cellules végétatives qui se sont agrandies et ont pris en même temps une coloration jaune-rougeâtre, elles restent fixées à la membrane commune. D'autres cellules appartenant à la même sphère ( $V$. globator) ou à d'autres familles ( $V$. minor) se transforment en anthéridies en se segmentant en 64 à 
128 anthérozoildes; ceux-ci sont jaunâtres, allongés, disposés en disques et munis de deux cils chacun, non à la pointe, mais à la base du rostre. Ils se meuvent d'abord dans l'anthéridie dont la paroi s'est formée pendant la segmentation, puis ils percent cette membrane pour nager dans la cavité coloniale; un ou plusieurs d'entre eux s'insinuent dans l'oosphère qui devient ainși oospore.

Une particularité remarquable se présente chez le Chlamydomonas pulvisculus. Après une série de générations asexuées, apparaissent des individus sexués; les mâles, formés au nombre de huit dans une cellule mère, sont plus petits notablement que les femelles qui ne naissent que deux ou quatre dans une cellule. Les uns et les autres possèdent deux cils et se distinguent de toutes les spores mobiles par la présence d'une membrane étroitement adhérente même dans la région postérieure; cette circonstance et leur long rostre ne permettent pas de confondre ces spores sexuées avec les individus ordinaires. Pour la copulation deux cellules différentes de sexe se soudent par leurs rostres, les cils tombent, la membrane se résorbe au point de contact et l'élément mâle pénètre dans le protoplasma femelle qui s'est contracté et avec lequel il se mélange. L'oospore s'entoure alors d'une membrane résistante dans l'intérieur de la cellule femelle toujours surmontée par la cellule mâle vidée.

Le Chlamydomonas pulvisculus se distingue donc des autres Chlamydomonas par ses sexes séparés, et de toutes les autres Volvocinées par l'existence d'une membrane, même chez les cellules sexuées; sous ce dernier rapport il présente une certaine analogie avec les Con. juguées.

Dans tous les cas précédents l'oospore ou la zygospore, résultant de la fécondation, s'entoure d'une épaisse membrane, prend une couleur rouge, s'agrandit lentement pendant quelque temps, puis entre dans une période de repos. A la germination l'exospore est rompue et le plasma de la spore s'échappe, entouré de l'endospore gonflée. Dans le Pandorina morum il donne une zoospore, rarement deux ou trois; dans les Stephanosphcera, il s'en forme deux à huit; ces zoospores deviennent le point de départ d'autant de familles. Dans le Volvox globator, au contraire, l'oospore donne directement une colonie. 
Malgré leur organisation fort simple, les Volvocinées présenten $t$ donc une assez grande diversité dans leur reproduction sexuée, et par ces deux caractères elles offrent un certain parallélisme avec les Conjuguées, vers lesquélles le Chlamydamonas pulvisculus peut servir de transition. Sous d'autres rapports, ces deux groupes sont en opposition au contraire ; car si les Volvocinées, pendant toute leur vie, sont douées de mouvements, les Conjuguées s'en trouvent complètement dépourvues. Si je les rapproche ici, c'est qu'ils peuvent être considérés tous deux comme des types peu adaptatifs, constituant deux petits rameaux attachés à la grande branche des $\mathrm{A}$ ]gues vertes.

M. Van Tieghem a signalé récemment un groupe spécial de Volvocinées, distinctes des précédentes par une matière colorante brune ajoutée à la chlorophylle, et par la disposition de leur matière gélatineuse (Synura, Syncrypta, Uroglena). Le Sycamina nigrescens s'éloigne encore davantage des Volvocinées; il ne possède point de chlorophylle, mais seulement un pigment noirâtre. Les cellules, entuurées chacune d'une épaisse membrane, se réunissent en grand nombre puur former des masses framboisées; les cellules extérieures de chaque masse sont munies chacune de deux cils que quelquefois elles perdent. Il n'y a de gélatine ni entre les cellules ni autour de la masse. La multiplication se fait par scission de la masse en ses cellules composantes qui s'éparpillent, puis se segmentent; leurs cellules filles peuvent s'isoler ou rester réunies en formant alors de nouvelles mûres. Enfin chaque cellule peut se transformer en chronispore par gélification de la membrane et concentration du protoplasma en un noyau réfringent, dépourvu de matière colorante. Dans des conditions favorables, cette spore se transforme en cellule négative ordinaire.

\section{PROTOCOCCACÉES.}

Les Protococcacées sont des Algues unicellulaires vivant isolées ou en colonies. Elles se distinguent des Volvocinées en ce qu'elles ne sont 
munies de cilis et de motilité qu'à certains moments de leur développement, et non durant toute leur vie. Elles se multiplient par zoospores qui peuvent copuler entre elles, conduisant aussi à la reproduction sexuée. On a fait une sous-classe spéciale, sous le nom de Palmella. cées, de celles de ces Algues qui se propagent en outre par scissiparité.

Erémobiées. Ce sont des Protococcacées vivant isolées. Les cellules sont souvent brièvement pédicellées, droites ou recourbées en $\mathrm{S}$, en spirale, etc. Pour la reproduction, le protoplasma de la cellule se divise en zoospores qui s'échappent et vont se disperser (Characium, Hydrocytium), ou qui restent fixées sur la cellule mère aux bords de l'orifice de déhiscence (Sciadium) pour y former de nouvelles cellules qui semblent des ramifications de la cellule mère; mais même dans ce cas au bout de trois ou quatre générations, de plus en plus petites, greffées ainsi les unes sur les autres, les zoospores se dispersent de nouveau dans le liquide ambiant. On a supposé qu'elles copulent alors.

Cette copulation entre zoospores a été observée (M. Klebs) dans l'intérieur même de la cellule mère chez le Chlorochytrium Lemnac qui vit dans les espaces intercellulaires des Lemna trisulca; les zoospores piriformes à deux cils forment ainsi, en se fusionnant deux à deux, des zygospores arrondies à quatre cils et à rostre très émoussé; celles-ci vont plus loin se fixer sur les feuilles de Lemna; elles s'entourent d'une membrane, et la cellule formée s'insinue dans l'intérieur de la feuille. Le cycle se répète pendant la belle saison; à l'approche de l'hiver, les cellules se transforment en chronispores à plasma dense et riche en amidon; au printemps suivant ces chronispores divisent de nouveau leur contenu en zoospores. Le Chlorochytrium Knyanum offre la plus grande analogie avec le précédent; seulement ses zoospores se développent sans copulation. Un autre genre voisin, l'Endosphara, semble constituer une transition vers les Palmellacées, en ce que son thalle unicellulaire se divise d'abord en cing ou six cellules d'où dérivent ensuite les zoospores. Dans le Phyllobium, endophyte du Lysimachia nummularia, les zoospores se distinguent déjà par leur taille en mâles et femelles; par copulation la microzoospore se fond completement dans la macrozoospore et il en résulte 
une zygozoospore n'ayant que deux cils; celle.ci produit, comme précédemment, dans l'intérieur des feuilles, une cellule; ce corps végétatif est ceperdant dẻjà plus compliqué ici, car il s'allonge en un filament ramifié.

On comprend encore dans ce groupe sous le nom de Protococcus de nombreuses Algues unicellulaires, vertes ou rouges, qui augmentent lentement de volume et divisent ensuite leur contenu en zoospores; il a été reconnu qu'un grand nombre de ces cellules ne représentent qu'un des états du développement d'Algues supérieures; de sorte qu'il est douteux qu'il existe réellement des formes indépendantes de protococcus.

Cœnobiées.-On désigne sous cenom les Protococcacées dans lesquelles les individus unicellulaires, d'abord isolés, se réunissent ensuite en famille; celle-ci peut affecter la forme d'un disque (Scenedesmus, Pediastrum), d'une sphère solide (Sorastrum), d'une sphère creuse (Calastrum), d'un réseau (Hydrodictyon).

Dans la multiplication asexuée le protoplasma de la cellule mère se divise en un nombre variable de zoospores ( 7 à 20,000 Hydrodictyon, 128 au plus Pediastrum) ou de masses immobiles (Scenedesmus) qui restent toujours enfermées dans une enveloppe formée par la cellule mère; les cellules filles se disposent ensuite en une colonie qui ne tardera pas à résorber la paroi commune, et qui n'aura plus qu'à s'agrandir, sans diviser ses cellules, pour constituer une nouvelle cœnobie adulte.

La reproduction sexuée n'a encore été observée que dans le gienre Hydrodictyon. Le protoplasma de la cellule mère se divise en 30 à 100 mille zoospores, plus petites que dans le cas précédent; elles s'échappent de l'enveloppe commune; deux, ou plusieurs même, se réunissent et forment par copulation d̀es zygospores à cils plus nombreux (M. Suppanetz), qui, venues au repos, deviennent sphériques et s'entourent d'une membrane épaisse; ce sont des chronispores. Après un repos de quatre à cinq mois, celles-ci s'accroissent lentement, puis divisent leur protoplasma vert en deux à cinq zoospores qui ne se meuvent que quelques minutes et se transforment bientôt 
chacune en une cellule polyédrique à angles prolongés en cornes, Les polyèdres augmentent de volume; leur contenu se divise en zoospores nombreuses qui, renfermées dans une mince enveloppe commune, s'échappent de l'épaisse membrane de la cellule mère, et, après s'être agitées environ pendant une demi-heure, se réunissent en un nuuveau réseau plus ou moins rudimentaire.

Palmellacées. - Dans ce groupe, les individus unicellulaires possèdent la faculté de se multiplier aussi par scissiparité; ils sont ordinai. rement réunis en colonies par une membrane gélatineuse commune. Nos connaissances sont encore imparfaites à leur sujet, et la reproduction sexuée, par copulation de zoospores, n'a encore été observée que dans le genre Tetraspora (M. Reinke).

\section{SIPHONÉES.}

Les Siphonées sont encore des algues unicellulaires : leur thalle, bien plus développé généralement que celui des Protococcacées, s'al. longe en filament plus ou moins ramifié; les rameaux qui restent en relation de continuité avec leur axe peuvent se différencier en rhizoïdes hyalins et quelquefois aussi en appendices foliaires. Ce thalle se relierait cependant par le genre Phyllobium à celui des Protococcacées; d'un autre côté les nombreux noyaux qu'il présente au moins dans un certain nombre de genres (Vaucheria, Valonia, Codium) et l'orifice de communication, entre l'axe et ses rameaux, souvent très rétréci, quelquefois même obstrué par un bouchon de cellulose, ne permettent pas de considérer comme absolue la distinction entre les thalles uni et pluricellulaires. Au point de vue de la reproduction il faut diviser les Siphonées en deux groupes suivant qu'elles sont pourvues ou non d'oosphères. 
Siphonées isogames.

Elles sont dépourvues d'oosphères immobiles, et se reproduisent par zoospores qui copulent au moins dans certains cas.

Dasycladées. -Le genre Acetabularia est le mieux connu (MM. Woronine, de Bary et Strasburger). Leur portion supérieure, pédicelle et chapeau, meurt chaque année; mais la partie inférieure (base du pied et rhizoïde) qui s'est séparée de la première par une cloison et qui s'est constitué une réserve alimentaire dans une vésicule basilaire, se développe de nouveau au printemps suivant; la base persistante du pied forme alors un nouveau filament sur lequel apparait ensuite un chapeau plus développé que celui de l'année précédente; quelquefois se forment deux chapeaux superposés. Les plantes âgées de quelques années déjà semblent seules produire des spores; cellesci apparaissent dans les rayons du chapeau et absorbent tout le protoplasma de la plante qui meurt ensuite. Les spores, au nombre de 30 à 80 par rayon, s'entourent d'une épaisse membrane et deviennent libres par la destruction du chapeau. Elles constituent en quelque sorte une génération sexuée; après un à trois mois, elles s'ouvrent par un opercule et laissent échapper les zoospores qui se sont formées dans leur intérieur. Les corps agiles d'une spore isolée périssent toujours sans rien produire; mais quand plusieurs spores se trouvent réunies, on voit leurs zoospores copuler par 2, par 3 ou même plus, et donner naissance à des zygozoospores munies de 4, 6, 8, 10 cils. Celles-ci, après avoir nagé un certain temps, s'arrondissent, viennent au repos et s'entourent d'une membrane; elles augmentent lentement de volume pendant plusieurs mois, puis donnent naissance à une plantule à large rhizoïde et à filament supérieurement terminé en pointe; la seconde année seulement la plantule porte un chapeau.

Le genre Dasycladus présente des verticilles rapprochés de rayons plus ou moins ramifiés et persistants. Sur les rayons se forment des cellules arrondies, pédicellées, qui sont des sporanges; c'est en effet dans ces cellules que prennent naissance les zoospores; celles-ci ne 
copulent pas entre elles, ni mème avec les zoospores d'un autre pied quelconque, mais seulement avec les zoospores de certains autres pieds de Dasycladus (M. Berthold); il semble donc y avoir des plantes mâles et des plantes femelles.

Botrydium. - Par sa reproduction, bien connue depuis le travail de MM. Rostafinski et Woronine, ce genre se rapproche surtout des Acetabularia: il vit dans les eaux douces et sur la terre humide sous forme de cellules vertes, arrondies, munies d'un prolongement rhizoïde hyalin. Un bourgeon latéral de la cellule donne naissance à un nouveau rhizoïde, puis s'isole de la cellule mère par une cloison; tel est le mode de propagation végétatif sur le sol. Menacées de dessiccation, ces vésicules se transforment en spores durables, munies d'une épaisse membrane; elles se conservent ainsi un an hors de l'eau. Quand ces hypro-sporanges, ou les cellules végétatives ordinaires, se trouvent placées dans l'eau, leur plasma se divise en zoospores à un seul cil qui deviennent libres par gélification de l'enveloppe commune, et qui ne donnent une plantule nouvelle que si elles peuvent atteindre le sol humide; maintenues dans l'eau elles s'entourent d'une membrane et la spore ainsi formée ne se développe encore en Botrydium que si elle rencontre le sol. Chez les individus adultes, le prolongement rhizoïde, plus développé et ramifié, attire à lui tout le protoplasma de la plantule, sous l'influence du soleil et de la dessiccation, et forme des cellules rhizoïdes, lesquelles, suivant les circonstances, se développent directement en nouveaux individus, en cellules durables (hypnosporanges) ou en sporanges ordinaires.

Outre ces divers modes de multiplication asexuée, les vésicules, pendant la saison chaude, transforment leur plasma en spores immobiles, vertes ou rouges, suivant les circonstances (soleil, humidité); ces spores, comme celles des Acetabularia, représentent la génération sexuée; elles donnent en effet naissance à des zoospores à deux cils qui, mises en liberté, se fusionnent à deux ou à plusieurs, pour produire des zygospores; celles-ci se changent en une nouvelle plantule; directement ou après une période de repos; dans ce dernier cas, elles 
s'entourent d'une épaisse membrane et deviennent irrégulièrement polyédriques

Codićes. - Ces plantes à thalle unicellulaire, mais abondamment et irrégulièrement ramifiées, simulent souvent un véritable tissu. Les sporanges sont formées par des branches spéciales à accroissement terminal limité qui s'isolent du thalle par une cloison et peuvent même se détacher (Bryopsis). Dans les Halimeda où l'on ne connaît encore qu'une seule espèce de zoospores, cette cloison n'apparaît même pas. Les Bryopsis et Codium (MM. Pringsheim, Cornu, Berthold) possèdent deux espèces de zoospores, séparées sur des individus distincts : les unes plus petites et jaınâtres, les autres plus grandes et vertes; chaque espèce isolée ne semble donner lieu à aucun développement ; cela est certain au moins pour les zoosporesjaunes. Le développement a lieu, au contraire, en réunissant ces corps agiles différents. Il y aurait done là des indications de sexualité; mais la copulation n'a pas encore été observée. Qu'elle provienne d'une fusion de zoospores, ou d'une zoospore unique, la cellule reproductrice donne en germant un rhizome plus ou moins ramifié, sur lequel s'élèvent ensuite d’es branches dressées.

Caulerpées. - C'est dans le genre Caulerpa que le thalle unicellulaire atteint sa plus grande différenciation. Jusqu'à présent on ne connaît qu'une propagation végétative au moyen de pousses latérales issues des rhizomes et devenues indépendantes par la destruction progressive du thalle.

\section{Siphonẻes oogames.}

Eilles se réduisent à la famille des Vaucheriacées, dans laquelle on renconire à la fois une propagation asexuée et une reproduction sexuée, de telle sortequ'à une série de générations agames succède une génération pourvue d'organes mâles et femelles; l'œuffécondé donne de nouveau une génération agame, puis le même cycle recommence.

Pour la reproduction asexuée le contenu de l'extrémité d'une bran- 
che renflée en zoosporange et séparée par une cloison du reste du filament, se contracte, acquiert une ou plusieurs vacuoles et s'échappe par une fente terminale dans le milieu extérieur. Cette cellule primordiale nue contient de nombreux grains de chlorophylle, sa couchè périphérique est au contraire hyaline et loge de nombreux noyaux dont chacun correspond à une paire de cils; ceux-ci couvrent toute la zoospore ou manquent quelquefois à ses deux extrémités antérieure et postérieure. En réalité cette grande spore mobile représente une colonie de petites zoospores réunies sous une enveloppe commune et présentent certaines analogies avec une famille de Volvocinées (MM. Schmitz, Strasburger). Là fente terminale du filament, étant plus étroite que la spore, celle-ci s'étire au passage et quelquefois même se rompt en deux parties dont l'une reste dans le sporange où elle continue à tourner (M. Sachs); chaque moitié peut alors se développer séparément. La zoospore arrive bientôt au repos, se revêt d'une membrane, puis germe dans l'espace de vingt-quatre heures, en développant un ou deux tubes et ordinairement un crampon rhizoïde rameux (Vaucheria sessilis, sericea, piloboloïdes). Dans la Vaucheria hamata la ofrande spore ne possède plus de cils; elle s'entoure d'une membrane dans l'intérieur de son sporange dont elle s'échappe encore par une fente terminale pour rester immobile au dehors jusqu'd̀ la germination. La spore imınobile de la $V$. geminata n'est plus mise en liberté que par destruction de la membrane sporangienne avec laquelle elle peut même se détacher. Dans la $V$. tuberosa la spore germe déjà dans son sporange. Les organes de la reproduction agame finissent donc par perdre leur mobilité.

Il peut y avoir une autre multiplication accidentelle par séparation de branches et génération des portions enlevées du thalle. Enfin, M. Stahl a observé que dans certaines circonstances le filament, devenu d'un vert plus brillant, se divise par des cloisons épaisses et gélatineuses en cellules d'égales dimensions; c'est le genre Gongrosira de certains auteurs. Ces cellules peuvent émettre un filament, comme une spore ordinaire; d'autres fois elles expulsent la totalité de leur protoplasma entouré d'une membrane mince, mais fragmenté déjà en corpuscules amiboïdes qui rompent ensuite la membrane commune, 
deviennent immobiles, s'entourent d'une membrane et germent; enfin res cellules à pseudopodes peuvent encore se transformer en cellules durables.

La reproduction sexuée s'opère par des cellules femelles ou oogones, et des cellules mâles ou anthéridies; toutes deux sont de courts rameaux issus du filament dont ils se séparent par une cloison; les organes des deux sexes se forment par paires, très rapprochés l'un de l'autre, quelquefois sur un pédoncule commun (V. racemosa). Dans la $V$. synandra un rameau renflé et séparé du filament par une cloison (androphore) porte de nombreuses anthéridies. Les anthéridies: dépourvues de chlorophylle sont droites, arquées ou recourbées en cornicules; leur contenu se divise en nombreux petits corpuscules allongés, munis de deux cils qui sont les anthérozoïdes; ceux-ci s'échappent par un orifice très rapproché de celui de l'oogone, quelquefois en outre par des ouvertures latérales indiquées d'avance par des papilles ( $V$. de Baryana, $V$. piloboloides). Les oogones sont de gros et courts rameaux à chlorophylle et à huile, ordinairement renflés obliquement en forme d'œuf; leur contenu vert et granuleux se contracte au centre de la cellule et se transforme tout entier en oosphère ( $V$. av̧ersa, $V$. rostellata), ou bien une portion incolore du protoplasma, située vers le sommet recourbé en forme de bec, se sépare de la masse principale et se trouve expulsée par l'orifice, lequel se forme par gélification de la membrane. Comme l'anthéridie s'ouvre en même temps, les anthérozoïdes arrivent dans l'oosphère et y disparaissent ; aussitôt après, celle-ci se montre entourée d'un contour très net; bientôt on y distingue une membrane à double contour. L'oospore devient rouge; son épaisse membrane se différencie en trois couches; après une période de repos, elle germe en produisant directement une plante asexuée, tandis que dans les autres Algues vertes oogames (Confervacées oogames), l'oospore donne naissance à des zoospores.

\section{CONFERVACÉES.}

Les Confervacées sont des Algues vertes à thalle pluricellulaire, formant un filament ou une lame (Ulvacées, Coléochétées); d'après leur Rietsch. 
mode de reproduction on peut les diviser en isogames et oogames.

Isogames. - La reproduction asexuée a lieu dans les familles isogames par des zoospores à quatre cils, plus grandesque d'autres à deux cils qui sont sexuées; ces zoospores sont formées en petit nombre (1, 2 ou 4 Ulothrichées), ou en grand nombre (Cladophorées, etc.) dans les cellules végétatives. Quelquefois le stade pélagique est supprimé et elles germent directement dans la cellule (Ulothrichées). On a aussi observé des chronispores agames; dans les Draparnaldia, il ne s'en forme qu'une par cellule végétative; elle peut rester enfermée dans celle-ci ou en sortir à l'état de grosse zoospore dont la vie pélagique est très courte. Plus fréquemment deux à quatre cellules durables prennent naissance dans chaque cellıle végétative, surtout à l'extrémité des rameaux (Stigeoclonium, Chaetophora endiviafolia); elles distendent le filament dont les cloisons transversales se détachent, de sorte que toutes les chronispores se trouvent réunies dans un tube non cloisonné.

La reproduction sexuće a lieu au moyen de petites zoospores sexuées à deux cils qui prennent aussi naissance dans les cellules végétatives, mais en plus grand nombre que les zoospores asexuées; elles copulent par deux ou trois (Ulothrichécs), puur former une zygospore qui, après quelques mois de repos, augmente lentement de volume, puis divise son contenu en deux à quatorze cellules, possédant une tache pigmentaire rouge ; ce sont sans doute des zoospores agames, quoiqu'on n'ait pas encore observé leur mise en liberté. La copulation des zoospores sexuées a encore été observée dans les Ulvacées, dans. les Cladophora arcta, sericea, dans les Chroolepus. Dans les Chactophorées, MM. Braun et Cienkowski ont seulement constaté l'existence des petites zoospores à deux cils; on suppose par analogie qu'elles sont sexuées et qu'elles copulent.

Une conjugaison particulière a été décrite par M. M. Cornu, dans l'Ulothrix zonata: le contenu d'une cellule végétative se divise en deux masses placées aux deux extrémités de la cellule ; elles s'avancent ensuite l'une vers l'autre comme deux zoospores et se réunissent vers le milien de la cellule en une masse unique dont la forme tend ver la 
sphère ; elle s'entoure d'une membrane épaisse et prend une couleur foncée; c'est une oospore.

Oogames. - Ces plantes habitent exclusivement les eaux douces. La reproduction asexuée est inconnue dans les Sphéropléacées; dans les deux autres familles plusieurs générations asexuées viennent toujours s'intercaler entre deux gẻnérations sexuées. Partout où la fécon dation a été constatée, l'oospore, après un temps de repos, divise son contenu en un certain nombre de cellules; celles-ci ou bien sont nues et deviennent directement des zoospores, ou bien sont séparées par des cloisons (Coléochétées), de sorte que l'oospore représente une véritable masse parenchymateuse, et le contenu de chaque cellule' cloisonnée ne se transforme qu'ultérieurement en une zoospore. Dans l'un et l'autre cas, ces zoospores issues de l'oosphère fécondée doivent être considérées comme une première génération agame, assez différente, il est vrai, des générations asexuées subséquentes. Les effets qui se manifestent dans les Coléochétées après la fécondation, ont une analogie évidente avec ceux que nous décrirons bientôt dans les Ricciées parmi les Hépatiques; dans l'un et l'autre cas, l'oospore fécondé se transforme en un corps cellulaire dont chaque cellule donne naissance finalement à une spore (M. Pringsheim). La différence essentielle, c'est que cette spore, mobile dans les Coléochétées, est immobile dans les Ricciées, ce qui correspond aux milieux habitées par ces deux familles, Le sporogone des Ricciées offre, il est vrai, un caractère de supériorité par sa plus haute différenciation, la couche extérieure restant stérile et servant à protéger les cellules fertiles intérieures; mais c'est là en somme une différence secondaire. Il y a encore une autre analogie, moins importante, il est vrai, entre ces deux familles. Dans les Ricciées, comme dans toutes les Cryptogames à archégones, ceux-ci s'accroissent après la fécondation, de façon à envelopper toujours le jeune sporogone; dans les Coléochétées aussi, pour la première fois parmi les Algues, nous voyons l'effet de la fécondation ne pas se borner à l'oosphère, mais s'étendre aux cellules voisines qui viennent former une enveloppe autour de l'oospore. Les Coléochétées qui se rattachent de la façon la plus évidente aux autres Confervacées et qui peuvent 
être considérées comme le point culminant de la série des Algrues vertes, ont donc une très haute importance au point de vue des relations de parenté entre les Cryptogames inférieures habitant l'eau, les Algues, et les Cryptogames supérieures terrestres, les Muscinées et les Vasculaires.

Dans les $E$ dogoniées, le contenu tout entier d'ure cellule se transforme en une zoospore dont l'axe est perpendiculaire à celui de la cellule mère; elle est remarquable par une couronne decils limitant la région antérieure hyaline. La cellule mère s'ouvre par une fente circulaire; la zoospore s'échappe entourée d'une vésicule hyaline qui ne tarde pas à se déchirer; plus tard elle se fixe par son extrémité antérieure qui se transforme en rhizoïde, et elle constitue alors un filament ordinaire. Toutes les cellules végétatives des Coléochétées peuvent donner naissance à une zoospore à deux cils; dans le $C . p u l$ vinata, ce sont principalement.les cellules terminales des branches qui se transforment ainsi.

Il faut rapprocher des Coléochétées une algue endophyte découverte par M. Cunningham, dans les feuilles de Camellia, auxquelles elle est fort nuisible; c'est le Mycoïdea parasitica qui, physiologiquement, semble intermédiaire entre les Algues et les Champignons. Cette plante forme, à la surface des feuilles, un disque analogue à celui des Coléochétées; de ce disque émanent des filaments qui perforent la cuticule et pénètrent dans les tissu où ils produisent des disques secondaires. Des filaments nouveaux, issus de la surface extérieure de ces derniers, soulèvent et déchirent la cuticule, et viennent former au dehors des faisceaux. Ce sont les cellules terminales de ces filaments et de leurs courts rameaux qui se transforment en sporanges, dans lesquels naissent des zoospores à deux cils. Celles-ci, se fixant sur les feuilles de Camellia, reproduisent les disques superficiels. En outre, les disques internes se multiplient par bourgeonnement.

La reproduction sexuéc s'effectue toujours au moyen a'oosphères im. mobiles et d'anthérozoïdes. Dans le Spheroplea annutina, les cellules du filament, allongées et cylindriques, se transforment toutes en organes sexuels sans changer de forme; dans les unes, le contenu, devenu rouge, se divise en un très grand nombre d'anthérozoüdes 
allongés à deux cils; dans les autres il se contracte en plusieurs oosphères vertes, disposées irrégulièrement ou en une rangée unique: Dans les deux cas des orifices arrondis, formés par résorption, apparaissent dans la paroi cellulaire; les anthérozoïdes s'échappent alors de leurs anthéridies pour pénétrer dans les cellules à oosphères et pour fusionner avec celles-ci. L'oospore devient rouge et s'entoure de trois membranes, dont l'extérieure ne tarde pas à tomber, tandis que la seconde se montre plissée irrégulièrement; au printemps l'œuf donne naissance à 2-8 zoospores à deux ciḷs, encore rouges, qui se transforment par germination en autant de filaments verts (M: Cohn). Dans le Cylindrocapsa involuta que l'on peut réunir en une seule famille avec l'espèce précédente, des cellules végétatives isolées ou réunies en groupes, tout en prenant une coloration rouge, se divisent successivement en plusieurs cellules filles dont chacune donne une anthéridie à deux anthérozoïdes fusiformes. Ceux-ci deviennent lihres par gélification des membranes. D'autres cellules du même filament se renflent en boule; leur contenu se contracte en une oosphère unique avec' tache réceptrice hyaline, leur membrane se perfore d'un orifice. La fécondation n'a pas été observée jusqu'à présent. Les œufs devien. nent tantôt rouges, s'entourent d'une membrane épaisse et donnent des chronispores; tantôt ils germent de suite. On suppose que dans le premier cas il y a fécondation, dans le second parthénogenèse.

Les anthérozoïdes des $E$ dogoniées sont très analogues à leurs zoospores et caractérisés aussi par une couronne de cils; ils se distinguent par leur taille beaucoup plus petite. Les anthéridies sont des articles du filament plus courts et plus pauvres en chlorophylle, isolés ou superposés jusqu'à douze en série; chacune par une cloison longitudinale ou transversale se divise en deux cellules mères super ou juxtaposées; un seul anthérozoïde se forme dars chaque cellule. C'est encore par déboîtement circulaire du filament que ces corps agiles deviennent libres; il peut n'y avoir qu'un seul anthérozoïde par anthéridie (Edogonium curvum). Dans beaucoup d'Edogonium, dans toutes les Bulbochcote, chacune des anthéridies, donne naissance, non plus à un ou deux anthérozoïdes, mais à une zoospore spéciale, inter- 
médiaire comme volume entre les zonspores ordinaires et les anthéro. zoïdes. Cette androspore, après s'être mue quelque temps, vient se fixer sur un oogone ou près d'un oogone, et, donne en germant une plantule mâle naine, paucicellulaire, n'ayant qu'une seule ou même point de cellule végétative, et donnant naissance par ses cellules fertiles à autant d'anthérozoïdes. Les oogones peuvent être placés sur les mêmes filaments que les anthéridies ou sur des filaments différents ; ils se forment toujours aux dépens de la cellule fille supérieure d'une cellule végétative qui vient de se diviser; cette cellule fille se renfle aussitôt en sphère et se remplit d'un plasma plus abondant; plusieurs oogones peuvent être directement superposés. A l'époque de la fécondation le protoplasma se contracte en oosphère; sa région tournée vers l'orifice futur devient hyaline. L'oogone s'ouvre par un trou latéral ovale (Bulbochcete, plusieurs FEdogonium) ou en se déboîtant encore circulairement, et la partie supérieure du filament s'incline alors. Par l'ouverture béante proémine aussitôt une gelée hyaline qui s'organise en canal ouvert en forme de bec, par lequel pénètre l'anthérozoïde. Dans l'Oed. diplandrum, la tache réceptrice n'apparaît qu'à la suite du contact de l'anthérozoïde. L'oospore formée s'entoure d'une membrane ordinairement épaisse ou même munie de piquants (Oe. echinospermum); l'œuf prend une couleur rouge ou brune pour ne redevenir vert qu'à la germination. L'oogone se détache avec l'oospore; après la pérjode de repos, l'épaisse membrane de la spore est brisée, son contenu șort entouré d'une mince enveloppe et se divise en quatre zoospores qui deviennent libres par résorption de la paroi commune; bientôt elles se fixent, s'entourent d'une membrane et donnent chacune une nouvelle plante (MM. Pringsheim, Jaranyi),

Les organes sexuels des Coléochétées peuvent être réunis sur le même pied (C. pulvinata, divergens, soluta) ou séparés sur des pieds différents (C.scutata). Pour la formation des anthéridies, les cellules âgées du parenchyme peuvent se diviser par deux cloisons perpendiculaires en quatre cellules justaposées, chacune à un anthérozoïde ( $C$. scutata), et ces anthéridies peuvent être disposées en arc de cercle ou en anneau. D’autres fois, sur des cellules végétatives isolées, naissent deux à trois 
courts rameaux, en forme de bouteille; ils donnent encore chacun un anthérozoïde; ces éléments mâles sont arrondis ou ovales, et munis chacun de deux cils à l'extrémité supérieure. Les oogones se forment al milieu des filaments du disque, et sont disposés alors sur un ou plusieurs cercles concentriques, ou bien dans les cellules terminales des filaments dressés ( $C$. pulvinata, divergens). La cellule destinée à former l'oogone se renfle et donne naissance à un prolongement mince, filiforme, qui s'ouvre au sommet; le plasma de ce col s'échappe, tandis que celui du ventre de la cellule (archégone physiologique) se contracte en oosphère que les anthérozoïdes atteignent par l'intermédiaire du col. Après la fusion des deux éléments sexués, l'oospore s'entoure d'une membrane, et aussitôt le support de l'oogone émet un filament qui adhère étroitement à celui-ci; c'est le premier élérnent d'une enveloppe corticale, à la formation de laquelle prennent quelquefois part aussi les rameaux des filaments voisins. Dans les $C$. orbicularis et irregularis, qui sont aussi dépourvus de col à l'archégone, cette enveloppe est nulle ou rudimentaire; dans les Coleochacte dont le thalle est discoïde, l'enveloppe ne couvre que la moitié supérieure de l'oogone; chez les autres espèces du genre la cortication est complète. Plus tard le thalle meurt, l'enveloppe brunit et à cet état l'oogone passe l'hiver. Au printemps l'oospore augmente de volume et se transforme par segmentations en une masse de tissu parenchymateux; en même temps l'écorce s'effeuille et se détache; plus tard, de chaque cellule s'échappe une zoospore (carpospore), semblable aux zoospores ordinaires, et donnant toujours naissance à un thalle asexué. Les Coléochétées, sous le rapport de la reproduction, ne diffèrent donc des autres Confervacées que par la cortication et surtout par les cloisons solides de l'oospore (M. Pringsheim).

Dans le Mycoïdea parasitica (M. Cunningham), les cellules terminales de certaines branches se changent, quand la sécheresse est continue, en autant d'oogones; au-dessous se développent de minces rameaux anthéridiens (?) qui viennent appliquer leur cellule terminale contre l'oogone et la soudent avec lui. La fécondation n'a pas été observée, de sorte que l'on peut se demander si ces rameaux anthéridiens ne sont pas en réalité les premiers filaments de l'enveloppe corticale, 
assez lâche, qui vient ensuite entourer l'oogone. L'oospore, imprégné d'eau au bout de quelque temps, donne un certain nombre de zoospares analogues aux zoospores ordinaires.

\section{CHARACÉES.}

Les Characées ont été rapprochées tantôt des Algues, tantôt des Cryptogames à archégones ; en réalité, elles n'ont de parenté étroite ni avec les unes, ni avec les autres; mais en somme, c'est encore avec les Algues qu'elles ont le plus d'analogie, et on peut les considérer comme un groupe aberrant de cette grande division.

Les Characées sont dépourvues de zoospores agames; leur multiplication asexuée a lieu :

Par corps tuberculeux ou nœuds souterrains à verticilles foliaires raccourcis, qui développent de nouvelles plantes par poussée latérale; par rameaux à base nue qui se forment sur les nœuds âgés ou coupés, et qui diffèrent peu, en réalité, des branches normales; par rameaux proemybryonnaires qui s'échappent, à côté des précédents, du nœud de la tige, et qui ont la même structure que le proembyron issu de l'oospore, lequel possède seulement en plus l'entrenœud d'où émare la racine principale.

Les Characées sont monoïques ou dioïques, suivant les espèces. L'anthéridie représente l'article terminal d'une feuille (Nitella) ou d'une foliole (Chara); sa paroi se compose de huit cellules appelées écussons; à l'intérieur de l'organe le centre de chaque écusson est occupé par une cellule cylindrique le manubrium, surmonté d'une autre cellule ou tête, qui porte à son tour six têtes secondaires. De chacune de ces dernières procèdent quatre longs filaments contuurnés, composés chacuns de 100 à 200 articles; chaque article donne naissance à un anthérozoïde enroulé en spirale et muni antérieurement de deux longs cils. A la maturité, les écussons se séparent et les anthérozoldes, mis en liberté, se répandent dans l'eau.

D'après des observations récentes c'est du noyau dẹ la cellule que 
dérive le corps de l'anthérozờde ; la partie centrale devient la vésicule, et Je plasma forme les cils (MM. Schmitz, Zaccharias).

Les Oogones sont munis d'une enveloppe (on a appelé le tout $\mathrm{Oo}_{0}$ gemme ou Sporogemme); ils émanent du dernier nœud du rayon principal terminé par l'anthéridie (Nitella), ou du nœud basilaire de la foliole qui donne l'anthéridie (Chara). Tout l'oogemme peut être considéré comme une pousse méthamorphosée. Une cellule basilaire qui correspond à l'entre-nœud inférieur de l'axe de cette pousse, supporte une courte cellule nodale de laquelle partent cinq filaments (feuilles) enroulés en spirale autour de l'oogone; celui-ci est relié à la cellule nodale par une cellule hyaline surbaissée (Chara) ou par un groupe discoïde de semblables cellules(Nitella). L'oogone ne contient qu'une seule oosphère, riche en plasma, en huile et en amidon, et à papille terminale hyaline: au-dessus d'elle les tubes enroulés constituent une couronne de cinq (Chara) ou de dix (Nitella) cellules; la couronne forme un col fermé en haut et latéralement par l'extrémité supérieure des cinq filaments, mais s'ouvrant à la mâturité par cinq fentes qui donnent accès aux antérozoïdes, Après la fécondation l'oospore se trouve entourée d'une envelope dure et noire provenant des filaments enroulés ; elle ne germe qu'après un temps plus ou moins long, et donne alors naissance à un proembryon consistant en une simple rangée de cellules; c'est aux dépens d'un des articles de ce proembryon que se développe la tige de la plante sexuée

L'oosphère semble cependant aussi capable, au moins dans certains cas, de développement parthénogénésique. Dans toute la presqu'île scandinave et en Allemagne, on n'a jamais trouvé que des individus femelles de Chara crinita, et cependant sette espèce annuelle est très répandue dans ces pays. Il faut rapprocher cette parthénogenèse, remarquable dans un groupe à organes sexuels aussi différenciés, du développement particulier des Fougères, découvert par M. Farlow et désigné par M. de Barry par le mot d'apogamie. 


\section{FUCOIDÉES.}

A côté de la grande série des Algues vertes, mieux représentées dans les eaux douces, il faut placer le groupe très important et exclu sivement marin des Fucoüdées, appelées aussi Mélanophycées. Elles ne se distinguent pas seulement des Chlorophycées par la présence, à côté de la chlorophylle, d'une matière colorante brune, la phycophéine, et par leur thalle, en général bien plus hautement différencié ; leur reproduction présente aussi (dans les cas connus), des caractères particuliers: les corps agiles portent leurs deux cils, non pas à la pointe, mais latéralement; la fécondation a toujours lieu en dehors de la plante mère, que les organes femelles soient du reste ciliés ou non; enfin l'oospore n'est pas une chronispore, mais se développe immédiatement.Les phénomènes de lafécondation sont encore inconnus dans une très grande partie de ces plantes; cela est d'autant plus regrettable que plusieurs des cas observés constituent des transitions intéressantes entre les copulations des żoospores et la fécondation d'œufs immobiles par des anthérozoïdes.

$\mathrm{Vu}$ l'état incomplet de nos connaissances, la classification de ce groupe ne saurait être considérée que comme artificielie. Nous examinerons successivement la reproduction dans les Phéosporées, Cutleriées, Tilopteridées, Fuçacées et Dictyotacées.

\section{Phéosporées.}

(MM. Thuret, Derbès et Solier, Rostafinski, Janczeiviski, Reinke).

Les corps reproducteurs sont tous des corps agiles qui se ressemblent entièrement, qquoique en réalité leurs fonctions soient différentes; car tantôt ce sont de véritables zoospores agames, et tantôt ils copulent entre eux pour produire une oospore. Toutes ces zoo.. spores sont formées dans des sporanges uniloculaires (nosporanges, Thuret) ou pluriloculaires (trichosporanges, Thuret). Dans les cas 
peu nombreux de copulation, observés jusqu'à présent, les zoospores sexuées provenaient de sporanges pluriloculaires; quand les mêmes plantes possèdent aussi des sporanges uniloculaires (Ectocarpus, Giraudia), les zoospores de ces derniers ne copulent point.

Certaines Phéosporées n'ont qu'une seule sorte de sporanges, uniloculaires (Laminariées, Asperococcus) ou pluriloculaires (Arthrocla. dia, Scytosiphon, Phyllitis, Colpomenia); plus souvent les deux sortes de sporanges se rencontrent dans les mêmes espèces (Punctaria, certaines Sphacélariées); ce peut être alors sur des individus différents, ou bien à des époques différentes de l'année, les uniloculaires apparaissant d'abord puis étant remplacés successivement par les pluiriloculaires (Ectocarpées, Mésogléacées). Suivant la position sur le thalle des sporanges uniloculaires, leurs zoospores s'échappent par la pointe ou par le côté. Dans les pluriloculaires ll peut y avoir un orifice terminal, et alors les zoospores s'échappent à mesure que les cloisons intérieures sont gélifiées (Ectocarpées, Mésogléacées), ou bien chaque compartiment s'ouvre séparément (autres représentants des mêmes fạmilles).

Les sporanges peuvent occuper des positions très variables, être localisés ou bien répandus sur tout le thalle, etc. Souvent ils proviennent de la cellule terminale de jeunes rameaux (Sphacelaria, Ectocarpus) dont l'accroissement se trouve aiors arrêté; la cellule, après s'être renflée en sphère, se divise en plusieurs étages superposés dont chacun se décompose en cellules par des cloisons perpendiculaires dans deux sens (Sphacelaria); plusieurs sporanges semblables peuvent être réunis sur un même rameau (Cladustephus). D'autres fois les rameaux s'allongent en filaments en tout semblables aux rameaux ordinaires, simples ou ramifiés, et ce sont alors des cellules de ces filaments, isolées ou réunies par groupes, qui donnent naissance aux zoospores (Ectocarpées). Enfin, les sporanges, souvent entremêlés de paraphyses (Laminaires) peuvent se grouper en sores répandus sur le thalle.

Dans les Sphacelaria on trouve encore des propagules particuliers sous forme de rameaux courts, pluricellulaires, munis d'un poil hyalin, qui naissent sur des branches ordinaires et se détachent à leur 
base; un même pédicelle peut produire successivement trois propagules; plus tard une de leurs cellules s'allonge en filament rampant, sur lequel nait une nouvelle plante par bourgeonnement latéral (M. Janczerwski).

La copulation de zoospores en mouvement a ité observée par M. Gœbel dans les Ectocarpus pusillus et Giraudia sphacelarioïdes. M. Berthold arriva à des résultats différents avec la première espèce; il constata que les zoospores se développent sans germination. Des résultats contradictoires ont été aussi obtenus dans d'autres circonstances par d'éminents observateurs, et cela suggère l'hypothèse que la fécondation, dans ces cas, est loin d'être un phénomène régulier, indispensable, qu'elle n'a lieu que dans certaines conditions extérieures, à des époques déterminées peut-être, entre des zoospores qui peuvent, ailleurs, se développer par parthénogenèse. Les observations de M. Falkenberg sur les C'utleria confirmeraient cette inanière de voir.

Dans l'Ectocarpus siliculosus et le Scytosiphon lomentarius, M. Berthold vit que les zoospores, provenant de sporanges pluricellulaires, identiques d'as pect, sont, en réalité, différents de sexe; et dans la dernière espèce, la plante elle-même tend à devenir unisexuée. En 'effet, de ces spores, les unes nagent pendant plusieurs heures, diautres arrivent au repos après quelques minutes; pour cela leur cil antérieur se fixe, attirant peu à peu le corps de la spore avec lequel il se confond, en même temps le cil postérieur se recourbe et disparaît aussi dans la petite masse plasmique; il s'est ainsi formé un œuf immobile, un peu allongé vers le point de fixation. Les zoospores, restées agiles, viennent s'assembler en grand nombre autour de lui, le frottant avec leur cil antérieur; au bout de quelques minutes l'une de ces spores malles s'est fondue avec la femelle immobile, qui s'entoure alors d'une membrane. Quelquefois aussi tous les mâles repartent sans qu'il y ait eu fécondation. L'œuf, non fécondé, ne tarde pas à rétracter son prolongement antérieur, à s'arrondir et à s'entourer d'une membrane; il germe parthénogénésiquement, mais son développement est plus lent que celui de l'œuf fécondé. Celui-ci donne des filaments rampants et ramifiés d'où proviennent les filaments ordinaires d'Ectocarpus; dans le cas du Scytosiphon, il se transforme en un disque qui représente 
peut-être la génération agame correspondant au Scytosiphon. Les zoospores mâles finissent aussi par venir au repos et par périr ; quelques-unes cependant vivent un peu plus longtemps, et formentalors une plantule faible et délicate.

On a observé quelquefois des oogones en germination dans l'intérieur d'un sporange; le stade pélagique de l'élément femelle se trouvait donc dans ce cas très réduit et même supprimé; n éanmoins la fécondation est loin de posséder ici ses caractères définitifs ; elle ne diffère même pas notablement de la copulation des zoospores capables de germer. Dans la famille suivante, nous allons faire un pas de plus vers la copulation typique.

\section{Cutleriacées.}

(MM. Reinke, Falkenberg).

Ce groupe fait partie en réalité des Phéosporées; il ne comprend que les deux genres Zanardinia et Cutleria.

Le cycle se compose d\& générations alternantes; l'une, asexuée, ne produit que des zoospores agames; l'autre, sexuée, donne naissance à deux espèces de zoospores différentes par leur taille et copulant entre elles. Ce sont les cellules superficielles du thalle ou de ses ramifications qui forment les oogones et les anthéridies, portés dans le genre Cutleria sur des individus différents. Ces sporanges ont la forme de courts cylindres, composés d'étages nombreux à deux cellules (anthéridies) ou d'étages peu nombreux à quatre cellules (oogones), chaque cellule donnant une seule oosphère ou huit anthérozoïdes. Les sporanges se trouvent groupés en sores sur des filaments, qui sont plus ramifiés dans les plantes mâles; dans les Zanardinia, les filaments sont, en général, plus simples, et portent les sporanges des deux sexes, les femelles étant terminaux.

Dans les Zanardinia, la génération agame ressemble à la sexuée; les sporanges uniloculaires donnent naissance à $4-6$ zoospores intermédiaires par leur taille entre les deux sortes de zoospores sexuées. Dans ce genre on connaît, de plus, une propagation végétative par 
pousses latérales. L'oospore des Cutleria forme, en germant, un thalle différent de la génération sexuée, mais très analogue aux Aglaozonia, qui ne produisent qu'une seule espèce de zoospores; aussi M. Falkenberg les considère-t-il comme la génération asexuée des Cutleria.

Dans les deux genres, l'anthérozoïde est beaucoup plus petit que l'oosphère; tous deux sont munis de deux cils dirigés, l'un en avant, l'autre en arrière. Isolés, ils périssent au bout de quarante-huit heures (mâles) ou de cinq jours (femelles); les oosphères s'entourent quelquefois d'une membrane. Réunis, ils conjuguent, et la germination a lieu : après s'être mue pendant quelque temps, l'oosphère se fixe par son extrémité hyaline qui devient la tache réceptrice, par laquelle seule pénétrera l'anthérozoïde; celui-ci se fond aussitôt avec l'oosphère et l'cuf ne tarde pas à se recouvrir d'une membrane, puis à germer.

\section{Tilopteridées.}

C'est un groupe encore peu connu, que son système végétatif rapproche surtout des Ectocarpées, tandis que, par ses organes reproducteurs, il senible plutôt parent des Fucacées. Les zoospores naissent dans des sporanges pluriloculaires analogues à ceux des Ecctocarpées; ces sporanges résultent de la transformation d'une branche tout entière du thalle (Haplospora) ou proviennent seulement de plusieurs cellules d'un filament unisérié (Tilopteris). 11 y a aussi des éléments de propagation, toujours immobiles, formés isolémentdansde grandes cellules du thalle, renflées en sphères; ils s'en échappent à la maturité, comme dans les Fucacées; quoique leur fẻcondation n'ait pas encore été observée, il semble cependant probable que ce sont des oosphères, et les zoospores pourraient être alors des anthérozoïdes. Les oogones où naissent ces oosphères, se forment, isolés ou réunis par deux ou trois, au milieu de courtes branches (Tilopteris) ou bien sur leurs côtés, et en simulant alors des rameaux (Scaphospora). 
Fucacées.

Les Fucacées sont dépourvues d'organes de propagation asexuée. La reproduction sexuée est parfaitement connue, grâce aux travaux de Thuret; sous ce rapport, la différence principale avec les Phéosporées, consiste dans l'immobilité des oosphères, qui sont dépourvus de cils, mais dont la fécondation n'a cependant jamais lieu dans les oogones.

Anthéridies et oogones naissent, entremélés de paraphy/ses, dans des cavités sphériques, les conceptacles, qui peuvent être hermaphrodites (Fucus platycarpus, Cystosira, Pclvetia, etc.) ou unisexués (Fucus, Himanthatia, Ozothalia); dans ce dernier cas, tout le thalle ne porte qu'une seule espèce de conceptacles, mâles ou femelles. Ces cavités communiquent avec l'extérieur par un orifice étroit, l'ostiole; elles sont fréquemment stériles, c'est-à-dire ne renferment que des paraphyses, alors plus abondamment développées. Les conceptacles peuvent être répandus sur toute la surface du thalle (Durvillcea, Splachnidium), ou bien ils se concentrent à l'extrémité de branches dichotomiques ou sur des rameaux latéraux d'une conformation particulière (Fucus, Ozothalia, Halidrys, etc ), ou sur des systèmes spéciaux de rameaux (Sargassum). Ils se développent dans le voisinage immédiat du point végétatif : une cellule ou plusieurs cellules, disposées en une rangée perpendiculairement à la surface et placées au centre du tissu destiné à former le conceptacle, s'arrêtent dans leur développement et meurent finalement; il se forme ainsi un court canal, qui s'élargit vers l'intérieur et finit par se transformer en une cavité sphérique; en même temps les cellules qui le limitent, se segmentent très activement. Des poils apparaissent dans la cavité; ils se ramifient de bonne heure dans les conceptacles mâles (M. Bower).

Les anthéridies naissent sur ces poils-rameux dont elles sont des branches latérales transformées; elles consistent en une cellule ovale, à paroi mince, dont le protoplasma se partage en nombreux petits 
anthérozoïdes pointus à une extrémité, munis d'une tache rouge et de deux cils. C'est par le sommet de l'anthéridie que les anthérozoïdes sortent; ils sont réunis en une petite masse, tantôt nue (Cystosira, Halidrys), tantôt entourée par la membrane interne de l'anthéridie (Fucus, Ozothalia) ; ils s'échappent ensuite du conceptacle par l'ostiole, guidés pour ainsi dire par les paraphyses proéminentes.

Les oogones se forment aux dépens de la cellule supérieure de poils bicellulaires, dont la cellule inférieure leur sert de pédicelle. Ils se renflent en sphère, et se remplissent d'un plasma sombre qui peut rester indivis (Pycnophycus, Himanthalia,Cystosira) ou se segmenter en deux (Pelvetia), en quatre (Ozothallia) ou en huit (Fucus) oosphères. Celles-ci remplissent d'abord tout l'oogone, dont la membrane externe est ensuite rompue; les œufs sortent, entourés encore par la membrane interne; ils s'arrondissent; puis, cette dernière enveloppe est à son tour rompue (Fucus) ou dissoute par l'eau (Ozothallia) ou gélifiée (Pelvetia); les oosphères sont arrivées alors hors du conceptacle, et deviennent accessibles aux anthérozoïdes qui se rassemblent en grand nombre autour d'elles, s'y attachent et leur communiquent sou. vent un mouvement de rotation. La fusion des deux éléments, quoique non observée directement, est cependant probable (M. Pringsheim); il est même très possible que plusieurs anthérozoïdes pẻnètrent dans l'œuf.

L'oospore s'entoure d'une membrane, se fixe à quelque corps solide et germe sans période de repos. Elle s'amincit à sa base, s'allonge en filament pluricellulaire unisérié, dont la cellule terminale se divise en quatre quadrants, origine du thalle, tandis que les cellules basilaires (prothalle de M. Rostafinski) donnent un crampon radiciforme; quand l'embryon a atteint à peu près un millimètre de longueur, son sommet se creuse en cuvette et donne naissance à un faisceau de poils; puis l'accroissement se ralentit considérablement. On n'a pu suivre plus loin le développement jusqu'à présent. Comme dans les Fucus adultes on voit fréquemment le sommet végétatif arrêter sa croissance par la formation d'une fossette poilue, il est possible que les germes observés jusqu'à présent, ne représentent pas le développement normal. 


\section{Dictyotacées.}

Tout en se rattachant itroitement aux Fucoïdées, les Dictyotacées peuvent, sous certains rapports, servir de transition vers le groupe suivant, celui des Floridées.

Les extrémités aplaties du thalle donnent naissance à trois formes de corps reproducteurs : des tétraspores et des cellules que, d'après leur forme, on suppose mâles et femelles, mais sans qu'il existe jusqu'à présent aucune certitude quant à leurs véritables fonctions; cette réserve faite, nous les appellerons, pour abréger, anthéridies et oogones. Chaque organe naît aux dépens d'une cellule épidermique qui proémine, se renfle, sépare par une cloison sa région basilaire étroite ; son extrémité supérieure devient la cellule réproductrice ; ces cellules peuvent être limitées à une seule face du thalle (Padina, Zonaria) ou apparaître sur les deux faces.

Les tétraspores, toujours formées sur des individus séparés, sont dispersées sur le thalle (Dictyota) ou réunies en groupes (Taonia, Padina); leurs quatre spores peuvent être disposées en tétraèdre ou comme les quartiers d'une sphère; leur nombre peut se réduire à deux et même à un. Devenues libres par gélification de la membrane commune à l'étát de cellules nues et immobiles, elles s'entourent bientôt d'une membrane et germent.

Les oogones peuvent être groupés en sores (Dictyota, Padina, Taonia). Le protoplasma de chaque oogone ne forme qu'un seul œuf nu et. immobile, qui se trouve expulsé et qui semble pouvoir germer sans fécondation. Dans les cas observés jusqu'à présent, œufs et tétraspores germent d'une façon identique, et tantôt reproduisent directement la plante-mère (Dictyota, Zonaria), tantôt forment d'abord une masse cellulaire, nœud central de $M$. Reinke, dont les cellules superficielles émettent des pousses identiques à la plante-mère (Taonia, Padina, Dictyopteris).

Les anthéridies sont toujours réunies en groupes, qui sont munis d'une enveloppe commune dans les Dictyota. Leur protoplasma, tout Rietsch. 
en se décolorant, se segmente dans trois directions perpendiculaires, et produit ainsi des anthérozoïdes immobiles, arrondis ou allıngés, qui deviennent libres par résorption de la paroi commune (Thuret, MM. Cohn, Reinke).

\section{FLORIDÉES.}

Le groupe très important des Floridées (MM. Bornet et Thuret) se compose en grande majorité d'Algues marines, remarquables par leur coloration rouge qui est due à la phycoérythrine, et par les particularités de leurs organes reproducteurs; la multiplication se fait au moyen de deux espèces de spores, les unes asexuées ou tétraspores, les autres résultant d'un acte sexuel ou carpospores, mais toutes deux immobiles, ainsi que les anthérozoïdes; ce qui est d'autant plus remarquable que toutes les Floridées habitent l'eau. C'est surtout dans l'acte sexuel que réside la caractéristique des floridées; si, dans certains cas, il offre des analogies évidentes avec ce que nous avons vu dans les Confervacées, il diffère en général très notablement de la fécondation typique, si uniforme ailleurs dans ses traits essentiels.

Tétraspores. - Ce sont des corps reproducteurs asexués et nus, différant des zoospores par l'absence de cils. Les tétraspores sont ordinairement portées par des individus différents de ceux qui produisent lescarpospores, rarement par les mêmes individus (certaines Porphyrées, Chylocladia, Callithamnion, Polysiphonia, Solieria); elles manquent, ainsi que tout autre corps reproducteur agame, dans les Lémanéacées. Dans la famille des Némaliées, les individus sexués peuvent porter aussi des spores ne résultant pas d'une fécondation, mais ces spores sont très différentes des tétraspores. Celles-ci naissent par segmentation d'une cellule mère et sont disposées en série, en tétraèdre ou comme les quartiers d'une sphère; leur nombre se réduit parfois à 2 ou à 1, ou dépasse le chiffre de quatre (Céramiacées). Les cellules-mères qui leur donnent naissance, terminent de courts rameaux latéraux (Callithamniées), ou sont nichées plus fréquemment dans le 
tissu même du thalle et recouvertes alors, le plus souvent, par une assise sterile; dans ce dernier cas, elles sont isolées ou réunies en sores. Les cellules mères peuvent aussi être localisées dans des branches spéciales du thalle qui prennent alors une confurmation toute particulière (Plocamium, de nombreuses Rhodomélées); rarement elles sont réunies en coussinets proéminents au-dessus du thalle (Gymnogongrus, Peyssonctia).

Tous les essais de culture des Floridées marines ont échoué jusqu'à présent: aussi ignore-t-on les relations qui existent entre les individus sexués et les asexués. Les premiers étant beaucoup plus rares, il est possible qu'une génération sexuée n'apparaisse qu'après une série de générations asexuées. Un certain nombre d'espèces, surtout dans la famille des Némaliées, ne sont connues qu'à l'état asexuée (Chantransia), d'autres seulement à l'ètat sexué (Helminthora, Némalion); l'hypothèse se présente donc tout naturellement de générations alternantes dimorphes, comme elles ont été constatées pour des genres voisins d'eau douce par M. Sirodot.

Une multiplication végétative par propagules semble exister dans le genre Monospora, sous la forme de courts rameaux à deux cellules dont la supérieure se détache et ne tarde pas à germer (MM. Bornet et Thuret); une multiplication analogue a été constatée aussi dans le Melobesacallit hamnioïdes par M. de Solms-Laubach.

Anthéridies (Thuret) - - Les anthérozoïdes sont immobiles et c'est l'eau qui les amène à l'organe femelle; on les appelle souvent aussi pollinides. Ils peuvent apparaître sur les mêmes individus que les organes femelles (p. ex.: Helminthora divaricata, Dudresnaya coccinea, D. purpurifera); mais en général, les Floridées sont diö̈ques. Les anthérozoïdes sont des cellules arrondies ou ovales, incolores; ils ne sont point nus, comme les tétra - et les carpospores, mais entourés d'une très mince membrane qui est la couche interne de la paroi de leur cellure mère; c'est par la gélification de la couche externe de la mềne paroi que l'élément mâle se trouve mis en liberté. Ces cellulus mères peuvent être isolées au sommet de rameaux di ou trichotomi.* ques, réunies en groupes à plusieurs étages (Porphyrées), disposées en 
verticilles sur l'axe principal (Dudresnaya); étroitement serrées, elles peurent recouvrir des portions isolées de la surface du thalle (Nitoplhyllum, Peyssonnelia, Halymenia); ou se trouver localisées dans des cavités formées par un repli du tissu ambiant (Melobesia, Gracılaria, Corallina); enfin les anthéridies se présentent encore comme des rameaux métamorphosés, ayant la forme de disques ou de massues (Rhodomélées).

Appareil femelle. - L'imprégnation se fait toujours au moyen d'un organe récepteur, le trichogyne qui ne prend, en général, aucune part à la formation du fruit ou cystocarpe. C'est sur le trichogyne que vient se fixer l'anthérozoïde; il y a soudure au point de contact, résorption de la paroi commune, puis l'anthérozoïde déverse son protoplasma qui se fusionne avec le protoplasma du trichogyne. Dans les cas les plus simples, c'est la partie inférieure de celui-ci qui se développe en fruit; très généralement le cystocarpe provient au contraire d'une ou de plusieurs cellules différentes du trichogyne, quelquefois attenantes à lui, mais presque toujours plus ou moins éloignées; la fécondation est donc indirecte. On donne le nom de trichophore ou d'appareil trichophorique à la cellule ou aux cellules qui supportent le trichogyne, celui de carpogone aux cellules qui se développent en cystocarpe après la fécondation.

L'effet fécondant provoqué par le protoplasma mâle, ne se manifeste donc pas nécessairement dans la cellule qui a reçu l'imprégnation; mais il peut se transmettre à des cellules plus ou moins éloignées qui fructifieront, tandis que la première cellule, le trichogyne, se fléchit et meurt; c'est là le phénomène spécial aux Floridées. Dans les Coléochétées la fécondation exerce une action secondaire sur les cellules placées près de l'oogone et qui viennent former une enveluppe au fruit; dans les Cryptogames à archégone, celui-ci redevient aussi actif à la suite de la fécondation; une excitation secondaire analogue se manifeste fréquemment dans les Floridées, et les cellules, placées dans le voisinage du carpogone, fournissent alors au fruit une enveloppe corticale.

Il peut y avoir deux trichogynes pour un carpogone (Porphyra, Ce- 
ramium) ou deux carpogones pour un trichnogne (Callithamnion). Ordinairement le trichogyne est allongé, capillaire, droit ou contourné en spirale (Dudresnaya); quelquefois aussi court et renflé (Batracho spermum), ou réduit à une courte proéminence (Porphyra); il fait toujours saillie à l'extérieur et sa paroi est épaisse, gonflée, gluante.

En dehors du cas très simple des Porphyrées, il y a deux 1ypes de développement pour le carpogone: tantòt, par des segmentations ré-pétées, il se divise en un corps cellulaire massif ou noyau (Dudresnaya); tantôt, après quelques segmentations peu nombreuses, ses cellules s'allongent en filaments rayonnants dans tous les sens, et il en résulte une tête fasciculée (Chantransia). Ordinairement chaque espèce ne présente que l'un ou l'autre mode; le Callithamnion corymbosum les possède cependant tous deux. Quand le développement, toujours centrifuge, du carpogone est terminé, ses cellules se transforment en spores, dites carpospores. Cette transformation est centrifuge; elle peut s'étendre à toutes les cellules du fruit (Némaliées, Céramiacées) ou se restreindre aux seules cellules superficielles (Polyides), laissant une masse centrale stérile, le placenta); entre ces deux cas extrêmes on trouve tous les intermédiaires. Dans cette transformation le volume des cellules augmente et elles se remplissent d'un plasma dense, riche en matière colorante.

Souvent le cystocarpe est dépourvu d'enveloppe corticale (Dudresnaya, Callithamnion, Chantransia, etc.). Quand elle existe, elle peut se composer de courts filaments unicellulaires et isolés (Némaliées) ou articulés et réunis latérnlement (Lejolisia); elle peut consister en un tissu résistant (Rhodomélées); quelquefois elle se trouve déjii ébauchee avant la fécondation (Batrachospermum, Rhodomélées).

Le fruit le plus simple se trouve dans les Porphyrées; les carpogones ressemblent aux autres cellules du thalle qui n'est formé que d'une seule assise ; les trichogynes se réduisent à de courts refoulements de cette cellule (une sur chaque face). La fécondation ne modifie ni la forme, ni le volume du carpogone qui se divise simplement par trois cloisons perpendiculaires en huit cellules disposées en deux étages; ce sont les carpospores. Ces cystocarpes si simples avaient été pris pour des octospores agames. Dans le Nemalion multifidum, tri- 
chogyne et carpogone forment encore une seule cellule; le fruit est plus compliqué. Les trichogynes des Lémanéacées proéminent à l'extérieur à travers l'écorce, dans l'épaisseur de laquelle demeurent compris Jes quelques cellules trichophoriques qui terminent les ramuscules. C'est la cellule attenante au trichogyne qui, seule ou avec ses vaisines, émet par bourgeonnement, dans la cavité annulaire du rameau, un faisceau de filaments moniliformes, ramifiés, enveloppés chacun d'une gaine mucilagineuse; tous les articles de ces filaments se transforment en carpospores.

Dans les Rhodomélées l'unique cellule trichophorique sépare déjà Je trichogyne des cinq cellules du carpogone. Dans le Callithamnion corymbosum (Céramiées) les deux carpogones unicellulaires sont séparés chacun du trichogyne avec une cellule trichophorique; ces deux cellules forment avec les carpogones un demi-tour autour du filament végétatif; trichogyne et trichophore disparaissent après la fécondation et il se forme ainsi deux cystocarpes distincts. Dans les deuxd erniers cas, la fécondation se transmet donc à travers une seule cellule.

Les organes de fructification des Corallinées se forment dans des conceptacles qui prennent naissance par creusement du sommet végétatif et rapprochement ultérieur des bords de la fossette; le fond des conceptacles à tétraspores est occupé par des paraphyses et des cellules allongées qui se segmentent transversalement pour produire les tétraspores. Les conceptacles à anthérozoïdes rappellent les spermoganies des champignons; les filaments anthéridiens proéminent par l'ostiole et portent à leur sommet 2 à 4 petites cellules, garnies chacune d'un faisceau de stérigmates; à l'extrémité des stérigmates les anthérozoïdes se forment par rétrécissement et se détachent emportant un fragment de leur pédicelle qui simule ainsi une queue. Les conceptacles femelles ont plutôt la forme de disques; les appareils femelles (procarpes) s'y développent en direction centrifuge et en grand nombre; ils sont composés d'un carpogone unicellulaire et d'un trichogyne. Ceux-ci sont, au centre du disque, renflés en massue à leur extrémité libre; à la périphérie ils sont beaucoup plus courts, rudimentaires, inaptes à la copulation. Par contre les carpogones du centre sont incapables de se développer en fruit. Ce sont cependant 


\section{$-71-$}

leurs trichogynes qui reçoivent l'imprégnation, à la suite de laquelle tous les carpogones du disque se fusionnent en une cellule carpogène unique qui porte à sa face supérieure tous les trichogynes; c'est seulement à sa périphérie que se développent les carpospores. Morphologiquement tous les procarpes du disque s'équivalent encore; physiologiquement ils diffèrent entièrement, ceux du centre n'étant plus que des trichogynes, ceux de la périphérie des carpogones; c'est au moyen d'une fusion que l'imprégnation se transmet du centre à la périphérie (M. de Solms-Laubach).

Dans les Dudresnaya, cette division des fonctions est encore poussée plus loin. Après la fécondation du trichogyne, quelques cellules de l'appareil trichophorique s'allongent en tubes connecteurs et vont transmettre la fécondation à la cellule terminale renflée de filaments fructifères voisins; ils se soudent à cette cellule et il y a fusion des deux protoplasma. Un seul tube peut transmettre la fécondation à plusieurs filaments. La cellule terminale des filaments fructifères se développe en cystocarpe. Cette singulière fécondation a èté découverte par MM. Thuret et Bornet qui lui ont donné immédiatement sa véritable interprétation, et qui l'ont encore rencontrée dans le genre Polyides. Depuis, des phénomènes analogues ont été retrouvés dans les Squammariées (Cruriopsis cruciata) où le tube connecteur se soude avec une cellule quelconque du filament fructifère pluricellulaire et où ce sont les autres cellules du même filament qui se transforment directement en spores (M.Schmitz). Plus récemment, une fécondation semblable a encore été reconnue chez les Halymenia Floresia, H. ulvoidea, Nemastoma, Grateloupia (M. Berthold).

Si donc, dans certains cas simples, la formation du fruit peut être comparée à ce que nous avons vu dans les Coléochétées, l'acte de la copulation devient ensuite de plus en plus indirect, de façon à différer complètement de ce qu'il est partout ailleurs, et les Floridées occupent, sous ce rapport, une place tout à fait spéciale parmi les Algues. La transformation du carpogone en cystocarpe reste, au contraire, comparable au développement de l'oospore des Coléochétées et des Ricciées. 
Germination et alternance de générations. - La germination des carpospores n'est pas encore bien connue. Dæns les Porphyrées, la carpospore, sans augmenter de volume, se transforme en un corps paucicellulaire, à membrane épaisse, dont les cellules donnent naissance à des filaments minces, ramifiés, pluricellulaires; on ignore le résultat ultérieur de ce développement. Dans cette famille, comme partout ailleurs, chez les Floridées, les carpospores sont mises en liberté à l'état de cellules nues et immobiles; quelquefois cependant, on les a vues animées de mouvements amiboïdes (Porphyra, Bangir, Helminthora). Elles s'entourent d'une membrane et germent sans période de repos, car on a constaté, quelquefois, un commencement de germination sur la plante mère. On ne sait rien de leur développement ultérieur qui est probablement fort lent.

Nous sommes mieux renseigné, fort heureusement, sur quelques Floridées d'eau douce, grâce aux travaux de M. Sirodot.

Dans le Balbiania (Chantransia) investiens, la carpospore donne naissance à un prothalle (protonema) rudimentaire, composé de filaments moniliformes ramifiés et portant des spores (sporules), qui germent en produisant la plante sexuée. Celle-ci se propage pour son propre compte au moyen de spores agames; mais elle possede aussi des organes sexués : anthéridies (pollinides) et carpogones avec trichogyne. Elle produit donc aussi des cystocarpes d'où proviennent des carpospores et ces dernières recommencent le cycle. Ainsi, il y a deux générations alternantes l'une sexuée, l'autre asexuée, celle-ci pouvant se reproduire d'unefaçon indépendante; ces deux générations sont reliées entre elles par des spores asexuées de deux sortes, sporules et carpospores. En réalité, par comparaison avec d'autres groupes, les Muscinées, par exemple, le cystocarpe peut être considéré comme une génération, et alors nous en possédons trois: l'une sexuée, avec une propagation agame indépendante en dehors du cycle, et deux asexuées différentes, la première résultant directement de la fécondation.

Dans un genre voisin, la spore asexuée se trouve supprimée entre le protonema (Chantransia) et la génération sexuée (Batrachosper- 
mum) qui reste, tout d'abord, parasite du protonema et qui ne s'affranchit qu'ultérieurement, pour vivre d'une façon indépendante. Cetce suppression de spore n'a rien d'inadmissible, si l'on se rappelle que dans les Muscinées on peut obtenir artificiciellement ce résultat et faire produire directement un protonema aux cellules du sporogone. Il est vrai que c'est la carpospore et non la sporule que l'on supprime ainsi ; cela n'est pas une objection, car l'apogamie des Fougères démontre que l'onspore elle-même peut disparaître par un procédé semblable. Il y a donc condensation du développement des Batrachospermum par rapport au Balbiana; de plus, ici ce n'est plus la génération sexuée, mais l'asexuée, l'individu végétatif, qui se propage pour son propre compte au moyen de spores agames. Enîn, M. Sirodot a montré qu'une autre condensation encore était possible, que le cystocarpe, au lieu de donner des carpospores, pouvait produire un protonema (Chantransia), tout comme dans les Mousses.

Dans les Lemanéacées, la génération sexuée, l'individu fructifère nait aussi sans spore de l'asexuée, de l'individu végétatif, dont elle est parasite d'abord pour s'affranchir ensuite. Cét individu végétatif se compose de filaments cellulaires unisériés rameux; l'individu sexué en naît comme un rameau de structure beaucoup plus compliqué. Ces phénomènes correspondent entièrement à ceux des Batrachospermum; mais les deux générations ont perdu leurs spores agames, et il ne reste plus que les carpospores, résultant, ainsi que tout le cystocarpe, de la fécondation. Evidemment, les Mousses ne proviennent pas des Floridees; mais, au point de vue. de l'alternance des générations, il me paraît utile de comparer les premières aux Lémanéacées; il est bon aussi de constater que celles-ci, comme les Muscinées, ne conservent, de toutes les spores agames des Algues, que les seules carpospores résultant directement de la fécondation. 


\section{CHAMPIGNONS}

Le groupe des Champignons est plutôt morphologique que physiologique. On peut en effet les considérer comme des Algues à parentés multiples et quelques botanistes ont même dissocié la classe pour joindre ses subdivisions à différentes subdivisions d'Algues; d'un autre côté, leur lien commun consiste dans l'absence de chlorophylle, absence corrélative de la vie parasitaire. Ces conditions physiologiques, anormales pour ainsi dire, impriment néanmoins aux Champignons un certain cachet particulier qui ne permet guère, quant à présent, de dissoudre le groupe. Pour la reproduction, la caractéristique commune, qui semble bien une conséquence du parasitisme, consiste dans une dégradation générale, non seulement de la sexualité et des organes qui la représentent, mais aussi des divers organes de la multiplication asexuée. Cette dégradation peut être accompagnée, du reste, de la régression ou du développement du corps végétatif; less termes ultimes de la différenciation dans le premier sens semblent être les Saccharomycètes et les Myxomycètes, traités plus haut et se rattachant, en réalité, aux Champignons. Les Basidiomycètes, au contraire, possèdant un corps végétatif très hautement différencié, ne montrent plus ni sporanges, ni cunceptacles, mais de simples spores isolées, analogues aux conidies.

On peut, en général, distinguer deux phases dans le développement: la spore produit le mycélium directement ou par l'intermédiaire de spores secondaires; le mycélium, directement aussi ou par l'intermédiaire de sclérotes qui ne sont qu'un état mycélien particulier, donnent naissance au réceptacle fructifère qui produit de nouveau les spores par formation endogène ou exogène. Quelquefois, les choses se compliquent davantage et il y a de véritables générations alterna・sənt

Nous distinguerons trois grandes divisions auxquelles se rattacheront des groupes plus petits: les Phycomycètes, les Ascomycètes et Basidiomycètes. 


\section{PHYGOMYCE்TES.}

Les Phycomètes constituent un groupe assez hétérogène. Leurs affinités sont nombreuses; par les Chytridinées, ils se rattachent aux Myxomycètes, par les Monoblépharidées aux. Algues oosporées en général, par les Mucorinées aux Conjuguées, par les Pérénosporées aux Siphorées; la plupart de ces Champignons se rapprochent encore des Siphonées par la simplicité de leur corps végétntif unicellulaire. C'est la seule division des Champignons où la reproduction sexuée a été constatée d'une façon incontestable; les Monoblépharidées possèdent même des anthérozoïdes qui manquent dans toutes les autres familles. Suivant que dans celles-ci la conjugaison est égale ou inégale, on peut les ranger en deux séries : les oosporées et les zygosporées; et dans chaque série, par une suite de transitions, on arrive de la fécondation incontestable à la parthénogenèse. C'est, en effet, dans les Phycomycètes que l'on peut le mieux constater la perte graduelle de la sexualité, et, déjà dans l'intérieur de ce groupe, on rencontre des Champignons qui en sont totalement dépourvus. A la suite des Phycomycetes nous avons donc placé deux familles, les Entomophthorées et les Ustilaginées, qui montrent bien encore des fusions protoplasmiques, mais ces fusions ne se distinguent pas nettement des anastomoses mycéliennes si fréquentes chez tous les Champignons. Les Chytridinées occupent une place à part; on peut, cependant, par leurs zoospores, les rapprocher des Phycomycètes oosporées qui en possèdent également, tandis que les corps agiles disparaissent dans les zygomycètes et ne se trouvent plus dans aucun autre groupe.

\section{CIYYTRIDINÉES.}

(MM. de Bary et Woronine, Nowakowski, Sorokin, Cornes, Alf. Fischer).

Les Chytridinées se rattachent aux Myxomycètes, et servent d'un autre côté de transition vers les deux grandes séries de Phycomycè- 
tes, les Oospol'ées et les Mucorinées. Ces champignons se réduisent souvent à un sporange; d'autres fois celui-ci possède un prolongement rhizoïde non séparé par une cloison (Chytridium, Phlyclidium); le prolongement peut devenir une cellule distincte du sporange et alors se développer en haustorium ramifié (Rhizidium) ou même en mycelium touffu (Obelidium); d'autres fois encore les sporanges ne sont plus que des renflements d'un mycelium plus développé (Cladochytrium).

La reproduction asexuée a lieu par zoospores; prur leur formation de nombreux noyaux apparaissent dans le sporange, deviennent des centres d'attraction pour le protoplasma et constituent ainsi autant de zoospores; quelquefois une partie du plasma sporangien n'est pas employée et reste comme substance intercalaire. Tout le contenu du sporange peut aussi sortir en une seule masse, s'entourer d'une membrane au devant de l'orifice et former seulement alor's ses zoospores (Polyphagus). Le sporange peut s'ouvrir par un opercule (ectoparasites, Zygochytrium) ou par un tubequi vient se souder à la membrane de la cellule nourricière, la double paroi se résorbant au point de contact (endoparasites). Les zoospores s'échappent alors; leurs mourements rapides ont lieu au moyen d'un cil unique, fixé à leur partie postérieure plus dense, l'antérieure étant plus claire; le corps est allongé, souvent courbé. Les mouvements peuvent être saccadés, irr'éguliers. La zoospore se change bientôt en une cellule amiboïde, se fixe sur la plante nourricière et s'entoure d'une membrane; puis de cette cellule, futur sporange, émerge un rhizoïde ou un haustorium qui pénètre à l'intérieur de l'hôte (ectoparasites, Chytridium, par exemple). Dans les endoparasites (Olpidium, par exemple), la zoospore perfore la rnembrane de la cellule nourricière, pénètre à l'intérieur et constitue alors un plasmodium qui vit au milieu du plasma nourricier et l'assimile; plus tard il s'entoure d'une membrane; il se change ainsi ordinairement en sporange. Dans les Synchytrium, au contraire, il se forme par ce procédé une cellule durable à membrane épaisse; après un temps de repos, sa paroi se perfore d'un orifice étroit, par lequel le contenu tout entier sort, enveloppé d'une mince membrane, pour constituer un sore; son contenu se divise, en effet, en nombreuses 
petites masses qui, s'entourant chacune d'une membrane, deviennent autant de sporanges; ceux-ci forment chacun un grand nombre de zoospores.

La copulation a été observée entre zoospores (Tetrachytrium). Une fusion de cellules égales, tout à fait analogue à celle des Mucorinées, est connue dans le genre Zygochytrium. Dans les Polyphagus, il y a conjonction entre deux masses plasmiques de volume inégal, qui proviennent de deux individus différents; il se forme ainsi une grande zygospore (chronispore) qui se comporte à la germination comme les sporanges ordinaires.

Il y a un groupe intéressant de Chytridinées agames, parasites des Saprolegniées ; il a été découvert et soigneusement décrit par M. Cornu; un travail plus récent de M. Fischer n'a ajouté que quelques détails.

Olpidiopsis. - Les sporanges et les spores durables sont contenus ensemble dans l'intérieur de certains filaments renflés et non cloisonnés de Saprolegniées. Les sporanges sont libres dans ces filaments; leur paroi va se mettre en contact avec celle de l'hôte par un tube qui perfore cette dernière et s'ouvre à l'extérieur; les zoospores s'échappent par ce canal. Les chronispores sont échinées, brunâtres, et sur leur flanc se trouve ordinairement accolée une petite cellule adjacente qui est vide et dont on ignore la signification; ces spores durables sont en réalité des sporanges qui s'ouvent. au dehors absolument comme les sporanges ordinaires; il n'y a point d'alternance de générations entre les deux formes. Chaque zoospore, en pénétrant dans la plante, devient un centre d'attraction pour le protoplasma de l'hôte, et donne un sporange de l'une ou l'autre forme.

Woronina. - Chaque zoospore donne naissance, non plus à un sporange, mais à un sore composé de plusieurs sporanges. Le protoplasma de la zoospore, moins individualisé que dans le cas précédent, se fond avec celui de l'hôte; il se constitue ainsi un plasmodium qui remplit un des compartiments d'un filament septé de Saprolegnia et qui se divise en plasmodes partiels dont chacun forme un sporange (sphérule). Ceux-ci prennent un aspect écumeux et se segmentent en 
globules qui deviendront des corps agiles; ils sont au nombre de vingt à trente par sporange et s'en échappent pour nager quelque temps dans le filament; finalement ils sortent de celui-ci et deviennent libres.

Les organes durables ne sont plus des sporanges, mais des sores; M. Fischer les considère comme un produit de transformation des états jeunes des sores ordinaires. Chaque zoospore pénétrant dans les Saprolegnia forme un sore de l'une ou l'autre espèce; les sores durables consistent donc en une série de cystosporanges (ou sporanges enkystés) dont chacun fournit encore, après repos, plusieurs zoospores.

Rozella. - La zoospore forme, avec tout le protoplasma d'un filament, un plasmodium unique qui se divise encore en plasmodies partielles; mais celles-ci se séparent par des cloisons. Le filament entier correspond donc ici à un des compartiments de Woronina; seulement les sporanges d'un même sore sont séparés maintenant par des cloisons, et constituent une série septée. La paroidu sporange ne se forme que quand la plasmodie partielle remplit tout son compartiment; cette paroi s'applique étroitement contre celle du filament de Saprolegnia qui paraît alors double. Les sporanges se vident par une perforation de cette double paroi et successivement de haut en bas. Chaque sporange peut être remplacé par une chronispore; mais comme la germination de celles-ci n'a pas été observée; on ne sait pas si ces spores durables sont encore des sporanges comme dans le cas précédent.

OOSPORÉES.

\section{I. - Reproduction sexuée.}

Monoblépharidées. - Les Monoblépharidées (M. Cornu) sont les seuls Champignons possédant une fécondation par anthérozoïdes; elles occupent donc une place à part dans ce vaste groupe, et semblent former, de même que le genre Mycoïdea, une transition vers les Algues. 
Les anthérozoïdes naissent, au nombre de cinq ou six, dans de petits sporanges spéciaux; ils sont ovales, triangulaires et munis postérieurement d'un cil unique; ils ne se distinguent, du reste, des zoospores que par leur taille plus petite; après s'être échappés de l'anthéridie, placée au-dessous, sur ou à côté de l'oogone, ils rampent à la surface de celui-ci, atteignent son orifice et finissent par pénétrer dans son intérieur. Les oogones, souvent superposées en nombre variable, ne forment chacun qu'une seule oosphère et s'ouvrent à la maturité; l'oosphère est alors remplie de globules oléagineux et présente une tache réceptrice; après la fécondation l'oospore s'entoure d'une membrane épaisse, soit à l'intérieur de l'oogone, soit après en être sortie.

Péronosporées. - La fécondation ne se fait pas par des anthérozoïdes; c'est l'anthéridie elle-même qui vient se mettre en contact avec l'oogone. La fusion des deur protoplasma a été constatée dans un certain nombre de cas; dans les autres elle est très probable. L'oogone ne constitue qu'une seule oosphère et n'emploie pas pour cela tout son protoplasma.

Pour la formation des oogones, certaines branches du filament se renflent à leur extrémité, rarement en leur milieu, en une sphère qui se remplit de protoplasma et qui se sépare par une (ou deux) cloisons du reste $d u$ filament. Un rameau issu du même filament au-dessoús de l'oogone, ou issu d'un filament voisin, se dirige vers cet organe et vient appliquer contre lui son extrémité renflée qui se sépare bientôt du rameau par une cloison et constitue ainsi l'anthéridie; rarement il se forme deux anthéridies pour un oogone. Quand celui-ci a atteint ensuite son volume définitif, le protoplasma qu'il renferme se diffẻrencie en épiplasma périphérique et en une oosphère remplie ảe goultelettes graisseuses; une fine branche émanée de l'anthéridie, le filament fécondateur, perce la membrane de l'oogone et pénètre jusqu'à l'oosphère. M. de Bary distingue alors trois cas :

$1^{\circ}$ Pythium. - Le protoplasma de l'anthéridie se divise en une couche pariétale, le périplasme, et un amas central, le gonoplasme. Le filament fécondateur s'ouvre largement au point de contact avec l'oo- 
sphère; le gonoplasme se transporte lentement, mais complètement dans l'œuf; il y a donc une réelle copulation;

$2^{\circ}$ Phytophthora omnivora. - Il n'y a point de distinction nette en périplasme et gonoplasme; il n'y a point d'orifice large au point de contact du filament et de l'oosphère; il doit cependant se produire une petite ouverture; car une partie du protoplasma mâle émigre dans l'œuf, la majeure partie restant dans l'anthéridie;

$3^{\circ}$ Peronospora. - Le filament fécondateur se renfle au point de contact avec l'œuf; on ne distingue aucun orifice; on ne voit point de migration d'une masse plasmique. M. de Bary pense cependant qu'une faible partie du protoplasma mâle doit passer dans l'œuf directement ou par osmose.

L'œuf, dans tous les cas, s'entoure ensuite d'une membrane d'abord mince, puis épaisse, et l'épiplasma vient le recouvrir d'une deuxième enveloppe. L'oospore passe ainsi l'hiver et ne germe qu'au printemps suivant, en donnant alors des zoospores (Cystopus) ou en formant directement un mycelium (Peronospora).

Saprolégniées (M. Cornu). - Le protoplasma tout entier de l'oogone se transforme en une ou plusieurs oosphères (jusqu'à trente-cinq dans l'Achlya); la fécondation (sauf dans le Pythium, rangé par M. de Bary, dans les Péronosporées) n'a jamais été directement observée; dans une partie des Saprolégniées, elle n'a certainement pas lieu. L'oogone se forme comme dans la famille précédente, sa surface se couvre de papilles copulatrices, quelquefois allongées en tube, souvent ouvertes au sommet; dans ce dernier cas l'oogone est muni de perforations. Il peut y avoir deux à quatre oogones superposés en série. Les anthéridies se forment au-dessus ou au-dessous de l'oogone, quelquefois sur l'oogone; elles peuvent aussi naitre sur une branche différente. J'extrẻmité libre de l'ébauche d'une branche anthéridienne se renfle aussi parfois et se transforme en oogone avec oospores, et alors des rameaux de la même branche constituent de nouvelles anthéridies (Achlya) souvent les branches anthéridiennes avortent, ou même ne se for- 
ment pas; mais elles viennent aussi fréquemment s'appliquer sur l'oogone et émettre un filament fécondateur, comme dans la famille précédente. M. de Bary distingue encore ici trois cas qui forment avec ceux des Péronosporées une série continue, laquelle conduit, par transitions successives, de la fécondation certaine à la parthénogenèse.

$4^{\circ}$ Plusieurs Saprolegnia, surtout S. ferox, forme monoica, Achlya, Aphanomyces. L'extrémité renflée de l'anthéridié émet un filament fécondateur qui s'allonge au-dessus de l'oosphère ou des oosphères, mais sans que l'on puisse distinguer aucune fusion entre les deux protoplasmes.

$5^{\circ}$ Dans certains Saprolegnia (torulosa, asterophora), les anthéridies s'appliquent souvent encore contre l'oogone, mais ils n'émettent point de filaments, ou ceux-ci n'atteignent pas les oosphères.

$6^{\circ}$ Souvent dans le genre Aphanomyces, et dans certaines especes de Saprolegnia, les oosphères de l'oogone se changent en oospores, sans qu'il y ait eu formation d'anthéridies. C'est la règle dans les S. Thureti, S. torulosa.

L'œuf s'entoure d'une membrane et ne germe souvent qu'après quelques mois. M. Pringsheim pensait que les oosphères féconảées avaient besoin d'un temps de repos plus long que les oospores parthénogénésiques; mais, d'après $M$. de Bary, ce temps de repos est très variable, non seulement suivant les espèces, mais aussi dans une même espèce et dans les oospores d'un même oogone.

A la germination, l'oospore donne des zoospores, ou un mycélium plus ou moins développé portant les zoosporanges. Dans le premier cas les zoospores sortent souvent ensemble de l'exospore réunies par une mince enveloppe commune, à l'intérieur de laquelle elles commencent parfois à germer.

\section{II. - Reproduction asexuée.}

Elle a lieu, en général, au moyen de zoospores; cependant ici encore il y a des diffẻrences assez notables. 
Dans les Monoblepharis les zoospores sont semblables aux anthérozoïdes, mais deux fois plus grandes; elles ne possèdent qu'un cil unique postérieur. Le corps de la zoospore sort le premier du sporange, son cil restant engagé; les efforts qu'elle fait pour dégager ce cil aident la sortie de la deuxième zoospore et ainsi de suite. Plusieurs sporanges peuvent se former à l'extrémité du même filament et être alors emboîtés les uns dans les autres, ou bien le second reste audessous du premier avec orifice latéral, etc. Leur formation est analogue dans les Saprolegnia. Dans les Achlya ils apparaissent quelquefois au sommet d'un filament qui se prolonge au-dessus d'un oogone.

Les zoospores des deux autres familles ont deux cils latéraux (Péronosporées, Saprolégniées) ou antérieurs (Saprolégniées); elles se forment dans des sporanges qui germent quelquefois directement, et qui se réduisent alors en fait à une spore. Dans les Pérénosporées, la vie pélagique de la zoospore peut avoir lieu en deux temps et être coupée par un intervalle de repos, pendant laquelle la zoospore s'entoure d'une membrane.

Les sporanges de plusieurs espèces de Peronospora se réduisent à de véritables conidies, formées à l'extrémité des branches et rameaux du mycélium; ces conidies en effet germent directement en donnant un filament. Dans les $P$. densa et $P$. macroscarpa le protoplasma de la conidie s'échappe de l'exospore,'puis s'entoure d'une nouvelle membrane au devant de l'orifice de la conidie et germe en formant alors son filament. Dans le genre Pythium le contenu du sporange, après s'être échappé de celui-ci entouré d'une mince membrane, s'arrondit encore près du sporange ouvert, et se divise en nombreuses zonspores; celles-ci sortent par un orifice de leur enveloppe commune, errent quelque temps, s'entourent d'une membrane et germent. Dans les Cystopus et Phytophthora, c'est à l'intérieur du sporange que la division a lieu; les zoospores du premier genre sont si intimement unies encore à leur sortie que l'on dirait une masse unique avec de nombreux culs; ces zoospores s'isolent et se dispersent ensuite. M. Leitgeb a récemment signalé une Péronosporée ncuvelle, Completaria complens, parasite sur les thalles de Fougères, à corps végétatif réduit à 
une cellule unique; elle ne se reproduit que par des conidies et chronispores.

La famille des Saprolégniées offre des dispositions plus compliqu uées que la précédente. Le genre Saprolegnia peut servir de transition; ses zoospores se forment à l'intérieur du sporange dont ils s'échappent un à un par une fente apicale ; après une vie errante de courte durée, ils s'entourent d'une membrane pour germer. Dans les Achlya et Aphanomyces le contenu du sporange se divise en masses plasmiques arrondies qui sortent ensemble, mais s'arrêtent à l'orifice pour s'entourer chacune d'une membrane; elles restent ainsi immobiles plusieurs heures, réunies en amas; puis chacune s'échappe à l'état de zoospore de sa membrane qui reste près de l'ouverture de déhiscence du sporange. C'est à l'intérieur du sporange que, dans les Dictyuchus les mêmes masses s'entourent de leur membrane; plus tard elles s'échappent encore à l'état de zoospores; celle des $D$. monosporus, $D$. Magnusi, $D$. polysporus perforent la paroi du sporange et les membranes abandonnées forment alors un réseau délicat qui remplit celui-ci. Dans le $D$. clavatus la paroi sporangienne se gélıfie pour laisser échapper les zoospores dont elle tient les membranes réunies en un amas lâche. Quelquefois aussi dans cette famille les zoospores s'échappent directement, arrivent alı repos après avoir nagé quelque temps et forment seulement alors leur membrane, qu'elles quittent bientôt pour passer par une seconde phase pélagique.

MUCORINÉES OU ZYGOSPORÉES.

(MM. Van Tieghem, Brefeld, Lemonnier, Bainier, Klein).

Les Mucorinées, appelées aussi Zygomycètes, sont caractérisées par leur reproduction sexıée qui consiste en une conjugaison de cellules égales et immobiles, et qui par conséquent est analogue à celle des Conjuguées. La fusion des filaments mycéliens a été observée très fréquemment dans ce groupe. M. Van Tieghem a aussi vu se fir 
sionner les spores, mème en grand nombre, d'où il résulte alor's une espèce de plasmodie.

Reproduction sexuće. - C'est ordinairement après avoir produit pendant longtemps des spores asexuées, et quand l'oxygène manque, que le mycélium se met à former des zygospores, qui semblent résulter d'un étouffement du mycélium par lui-même. Deux filaments presque toujours semblables, droits (Mucor, Rhizopus, Chaetocladium) ou arqués en mors de pince (Phycomyces, Piptocephalis) se touchent par leur extrémité qui s'est renflée et séparée an moyen d'une cloison du reste du filament; Ja paroi commune se résorbe et les deux cellules fondent leur plasma en une masse unique qui s'accroît et se revêt d'une épáisse membrane cartilagineuse, hérissée de bosselures et de pointes; le tout est recouvert par la paroi noircie des cellules mères. Les filaments copulateurs peuvent produire des épines noires, creuses, dichotomiques, qui revêtent l'oospore d'une double couronne touffue (Phycomyces nitens) (M. Van Tieghem). Dans les Mortierella (M. Brefeld) les zygospores se forment au milieu de l'épais feutrage de filaments; ce sont des tubercules brun jaunâtre entourés d'une épaisse capsule formée par les rhizoïdes voisins; des capsules partielles semblables se retrouvent dans le même genre autour des sporanges. Dans les Piptocephalis l'oospore ne remplit qu'une faible partie du volume des deux cellules et proémine au dehors.

Mais cette copulation ne semble pas indispensable à la formation de l'oospore dans un grand nombre de cas au moins (Absidia, Mucor, Sporodina, Spinellus). Souvent on voit un filament s'apprêter à la copulation, mais ne rencontrant pas le second filament copulateur, il n'en forme pas moins sa spore qui est une azygospore: «La différence sexuelle étant en effet très faible, si petite qu'à peine se traduit-elle au dehors par quelque marque apparente, on doit admettre qu'il manque bien peu de chose à chacune des deux cellules en présence pour qu'elle se suffise à elle-même. Ce peu qui lui manque, quoi d’ètonnant qu'elle puisse le tirer à la rigueur de l'appareil végétatif par voie d'osmose ou de nutrition, bien qu'elle le reçoive ordinairement par voie de fusion avec un autre corps proto- 
plasmique, doué de propriétés complémentaires des siennes.)(M. Van Tieghem, Ann., Sc. N. $6^{\circ}$ S. T. 4.) Certaines espèces de Pilobolus ne forment jamais de zygospores.

L'oospore ne germe qu'après un temps de repos.

Reproduction asexuée. - Ses organes sont variés; le mycélium peut en effet porter des sporanges, des chlamydospores, des stylospores, des conidies.

Un filament épais s'élève perpendiculairement en l'air attirant à lui la totalité ou la majeure partie du protoplasma mycélien qui passe finalement dans le renflement sphérique, développé peu à 'peu à l'extrémité de cette branche. Ce renflement se sépare de son support par une cloison et constitue alors le sporange ; son protoplasma se différencie en deux substances, l'une granuleuse (plasma sporigène) se divise en spores, l'autre homogène et brillante semble constituer les parois d'autant de petites cellules qu'il y a de spores. Les petites masses formées par la première arrondissent leurs angles et secrètent une membrane de cellulose; leurs granules disparaissent, elles deviennent homogènes et réfringentes. Le protoplasma intersporaire occupe tous les espaces laissés entre les spores; il manque, quand il n'y a qu'une seule spore qui alors absorbe tout le contenu. Au lieu de sphériques (Mucorinées, Pilobolées, Mortierellées), les sporanges peuvent être allongés (Syncéphalidées), et alors simples ou ramifiés, et contenir des spores séparées encere par du protoplasme intersporaire. Au lieu d'être simple, le support se ramifie souvent plus ou moins, ses extrémités supportant autant de têtes; il se forme ainsi des systèmes de sporanges.

Le nombre des spores de chaque sporange est extrêmement variable, il peut s'élever à 50.000 et se réduire à l'unité. La déhiscence s'opère par résorption ou déchirement de la paroi, etc.; dans les Pilobolées tout le sporange est lancé en l'air. Le support quelquefois proémine dans l'intérieur de la cavité sporangienne. constituant la columelle. Fréquemment, à côté des sporanges, on rencontre des chlamydospores (Mucor, Mortierella), soit portées sur des pédicelles, soit renfermées à l'intérieur des filaments; des stylospores ont été signalées dans 
les Syncéphalidées, Mortierellées. Enfin M. Zopf a signalé l'existence de sporanges portant à la fois des 'spores endogènes et des conidies exogènes (Mucor aspergilloïdes). D'après M. Brefeld, les conidies sont des sporanges réduits, et de pareilles réductions qui se rencontrent dans tout le groupe des Champignons, peuvent être obtenues artificiellement par culture; entre les Mucorinées n'ayant que des sporanges (Mucor, Pilobolus, Rhizopus) et celles n'ayant que des conidies (Chaetocladium, Piptocephalis) il y a tous les degrés intermédiaires (Thamniduum, Chaetocladium, etc.).

Les spores agames, aussi bien que les zygospores (et azygospores), donnent des tubes sporangifères en germant dans l'air humide, et un mycélium en se développant dans un milieu nutritif. Les conditions de milieu déterminent donc seules le mode de germination (M. Van Tieghem). Du reste : «Ni l'œuf, ni la spore ne constituent une unité biologique. L'un et l'autre peuvent êtr: mutilés et fractionnès, sous de certaines conditions, sans que chaque fragment cesse de posséder toutes les propriétés génératrices. "

\section{ENTOMOPHTHORÉES.}

Les mycologistes ne sont pas d'accord sur la place à assigner aux Entomophthorées et aux Ustilaginées; les uns les considèrent comme voisines des Basidiomycètes; d'autres les rapprochent des Saprolégniées, d'autres des Mucorinées. Par leur mycélium unicellulaire les Empusa sont parentes en général des Phycomycètes. Les deux genres Empusa et Entomophthora causent des maladies épidémiques aux monches et aux chenilles (MM. Cohn, Brefeld, Nowakowski, Sorokin) dont lecorps se trouve envahi par leur mycélium, et perforé plus tard par de nombreuses hyphes qui sont ramifiées seulement dans les Entomophthora (où le mycélium est cloisonné). A l'extrémité de ces hyphes ou de leurs rameaux, se forme une spore qui est projetée; la place où git le cadavre de la victime se trouve ainsi entourée d'une quantité de spores; celles-ci ne conservent que très peu de temps leur 
faculté germinatrice (un ou deux jours) ; elles allongent leur mince membrane en un court filament dont l'extrémité renflée se sépare par une cloison et devient une spore secondaire ou sporidie; le protoplasma non employé à sa formation s'accumule près de la cloison et exerce une tension croissante; finalement la sporidie est projetée el cette masse plasmique qui l'entoure sert à la fixer, dans les circonstances favorables, à la face ventrale d'une mouche saine (Empusa), ou d'une chenille (Entomophthora). La sporidie émet alor's aussitôt un mince filament qui perfore le corps de la victime, et se renfle ensuite en une grosse cellule (Empusa) ou bien forme immédiatement ¿un mycélium (Entomophthora). Dans le premier cas la cellule initiale produit par un bourgeonnement latéral, analogue à celui des Saccharomyces, plusieurs cellules filles qui envahissent le corps adipeux et s'y multiplient rapidement de la même manière; plus tard toutes les cellules s'allongent en filaments unicellulaires; quand ceux-ci rencontrent la paroi du corps, ils s'y appliquent en renflant leur extrémité, dans laquelle s'accumule le protoplasma; finalement se forme une mince hyphe qui perce le corps, et le cycle recommence alors.

Dans les Entomophthora le mycelium s'accroît rapidement et en quelques jours envahit tout le corps. Cette propagation peut se faire en partie par conidies; plus tard il perfore encore la paroi du corps, pour aller former ses spores à l'extérieur. Mais à mesure que l'automne s'avance, les spores ordinaires font place peu à peu à des chronispores ou spores durables à membrane épaisse, produites à l'intérieur $\mathrm{d}, \mathrm{u}$ corps; en même temps le mycelium cesse progressivement de s'étendre en dehors de l'animal. M. Nowakowski était disposé à considérer ces chronispores comme le résultat d'une conjugaison, souvent scaliforme, analogue à celle des Conjuguées. Ce n'est pas tout à fait l'avis de M. Brefeld; d'après ce naturaliste, au point de formation des spores durables, les filaments sont enchevêtrés, souvent anastomosés, mais ces anastomoses, simplement végétatives, ne diffèrent pas de celles qu'on a trouvées souvent sur le mycélium des Mucorinées, des Ascomycètes, des Basidiomycètes, et que personne n’a jamais songé à considérer comme des actes sexués; des fusions semblables sont encore très fréquentes entre les stérigmates portant 
les sporidies et entre les sporidies elles-mêmes, ou encore entre les spores ordinaires (conidies) d'où proviennent ies sporidies. D'un autre côté ces chronispores se rencontrent aussi sur des filaments isolés, n'offrent aucune trace d'anastomose. Comme les épidémies ne se suivent qu'd̀ quelques années d'intervalle sur les mêmes chenilles, M. Brefeld pense que quelques années de repos sont indispensables à ces spores durables qu'il n'a pas réussi à faire germer.

M. Giard considère comme chronispores des Empusa, les spores que l'on trouve sur les ailes des mouches mortes victimes de ce champignon; elle germent encore deux mois après leur formation. D'un autre côté, M. Brefeld a rencontré des chronispores d'Empusa dans le corps de mouches autres que la mouche de chambre; ces spores durables apparaissent en automne après de nombreuses générations de spores ordinaires; c'est encore par l'abdomen qu'elles pénètrent dans le corps de leur hôte avec leur filament germinatif.

M. Brefeld assimile les spores durables des Entomophthorées aux zygospores des Mucorinées ou aux oospores des Saprolégniées. Si cette comparaison est exacte, l'acte sexuel qui déjà disparaît dans quelques espèces de ces dernières familles, est encore moins fréquent ici et en même temps moins caractérisé; il re se distingue plus en effet, dans bien des cas, de la simple anastomose entre filaments végétatifs; d'un autre côté, une copulation se fait entre les stérigmates et les sporidies qui résultent de la germination de ces spores durables. La sexualité devient donc ici diffuse et méconnaissable. Dans les Entomophthorées les chronispores, homologues des oospores, sont encore bien moins fréquentes que les spores ordinaires; dans la famille suivante, elles deviennent l'unique mode de propagation.

\section{USTILAGINÉES.}

(Tulasne, MM., Fischer von Waldheym, de Bary, Wolff).

Le genre Entyloma peut servir d'intermédiaire entre les Ustilaginées ei les Entomophthorées; avant les chronispores, il 
produit en effet encore des spores ordinaires qui disparaissent dans les autres genres où il ne reste plus que les spores durables. Cellesci rlonnent à la germination un promycélium avec sporidies; l'ensemble de cette formation constitue un support fructifère, qui disparaît encore partiellement dans les Ustilago. En somme il y a donc simplification dans les organes de propagation et tendance à leur réduction en une forme unique, les chronispores.

Les Ustilaginées sont des champignons vivant en parasites dans l'intérieur de divers végétaux, surtout des graminées (charbon); leur fructification s'opère toujours dans des organes déterminés de la plante hospitalière; ces organes se remplissent d'une poussière noire ou brune, composée seulement de spores innombrables à membrane épaisse; le mycélium finit en effet par disparaître.

Les chronispores se forment sur les filaments (Entyloma) ou à leur extrémité (Tilletia) et sont alors isolées. Dans les Tuburcinia (M. Wo. ronine) c'est l'extrémité d'un filament, ou de deux filaments accolés, qui se renfle en sporange que les hyphes voisines viennent envelopper d'une capsule. Dans les Urocystis leur formation est plus complexe; deux ou plusieurs rameaux se rencontrent, s'entrelacent étroitement en se contournant quelquefois en spirale et en renflant chacun son extrémité ; la pelote ainsi formée augmente de volume, les parois des filaments se gonflent, leur contenu devient réfringent; toute la pelote s'entoure d'une membrane brune qui, pénétrant à l'intérieur, divise la masse en compartiments. Des filaments voisins viennent appliquer étroitement contre cette pelote leur extrémité reuflée qui se sépare par une cloison et s'entoure aussi d'une mernbrane brune; ainsi se constituent les spores externes stériles de ces formations complexes qui sont en réalité des sporanges. A la germination, une ou plusieurs des spores internes seulement émettent autant de promycélium. Les chronispores des Geminella se forment encore d'une façon analogue, seulement les parois ne se gélifient pas, et les spores latérales stériles manquent. Dans les Ustilago il se forme encore une pelote analogue de rameaux entrelacés, laquelle ne s'entoure pas d'une épaisse membrane commune; les parois se gélifient davantage et finissent par être 
complètement risorbées par les spores qui restent seules sous forme d'une poussière noire; le mycélium disparaît.

A la germination, le promycélium sort de l'épaisse membrane de la spore par un orifice étroit (Entyloma) ou par une fente (Tilletia). Il constitue un court filament dont l'extrémité arrondie forme, dans les Entyloma, 4 à 8 rameaux allongés, minces, fusiformes qui absorbent tout le protoplasma; ils se séparent chacun du promycélium par une cloison, puis copulent deux à deux en forme de $H$; dans chaque paire, une des branches se prolonge et forme une sporidie recourbée qui se sépare encore par une cloison et se détache pour donner naissance aussitôt à un lung et mince filament germinatif; celui-ci pénètre dans l'hôte. Les stérigmates qui n'ont point copulé restent stériles; il y a donc encore ici une espèce de sexualité transportée de la chronispore aux rameaux qui en émergent. Dans les Tilletia les rameaux sont supprimés et les sporidies naissent directement du promycélium; elles copulent entre elles, mais moins régulièrement que les rameaux de l'Entyloma. Les sporidies associées en $H$ se détachent ensemble et émettent chacune un filament qui peut penétrer dans la plante ou former encore une sporidie secondaire. Mais contrairement aux Entyloma les sporidies primaires isolées germent également, c'est-àdire que la fusion est facultative. Le promycélium des Tuburcinia (M. Woronine) porte 4 à 8 sporıdies à son sommet qui se sépare par une cloison et se détache; c'est une baside; les sporidies copulent à 2 ou à 3 sur la baside; copulées ou non, elles peuvent donner des sporidies secondaires. Le promycélium des Urocystis se comporte comme celui des Tilletia. Dans les Ustilago, la spore émet un (rarement plusieurs) promycélium qui se divise en articles; tantôt ceux-ci donnent des sporidies par refoulement latéral, tantôt ils germent directement.

\section{ASCOMYCĖTES.}

Les champignons de ce vaste ordre possèdent, en général, un corps végétatif plus compliqué que dans les Pyrénomycètes; les organes 
de propagation sont extrêmement variés, et l'nn peut dans une même espèce rencontrer des conidies, des spermaties, des stylospores et des ascopores, les trois dernières pouvant être renfermées dans des conceptacles particuliers appelés spermogonies, pycnides et périthèces. Mais ce qui caractérise ce groupe, c'est la présence des asques ou sporanges particuliers, à l'intérieur desquels se forment les ascospores.

Les asques ou thèques sont ordinairement des filaments ou tubes allongés, contenant 8 , quelquefois $2,4,16$ ou encore plus de spores. Pour leur formation le noyau de l'asque subit des bipartitions répétées; autour des noyaux multiples qui en résultent, vient s'accumuler une partie du protoplasma; les spores ainsi formées se disposent en une rangée, s'accroissent du double et s'entourent d'une mince membrane; tout le plasma n'est pas employé à cette formation. Plus tard, la déhiscence a lieu de diverses manières, par opercule, par gélification de Ja paroi, par gonflernent du protoplasma restant non employé; les spores sont ordinairement projetées; dans les Tubéracées elles ne deviennent libres que par la destruction du fruit. Les asques sont réunis en fruit de conformation variable; très simple et dépourvu d'enveloppe dans les Gymnoascées, il devient plus compliqué dans les Périsporiacées où il est entouré d'une paroi corticale, sous laquelie se forment les asques non mélangés de paraphyses. Le fruit prend la forme d'une bouteille ou sphère, ouverte supérieurement par un ostiole dans les Pyrénomycètes; on lui donne alors le nom de périthèce; il contient à la fois des asques et des paraphyses; il en est ordinairement de même dans les Discomycètes oủ le périthèce s'ouvre largement et s'étale en une cupule ou même en un disque. Enfin dảns les Tuberacées le fruit arrive à une structure bien plus compliquée. L'ensemble des asques constitue l'hymenium, placé sur le tissu soushyménial.

Le développement de ce fruit a été très fréquemment observé, mais les botanistes sont loin d'être d'accord sur l'interprétation à donner aux phénomènes : pour les uns, les asques résultent, au moins dans un grand nombre de cas, d'une véritable fécondation; pour les autres, ils se forment d'une façon agame et si, dans quelques cas, on a observé 
des copulations entre filaments, elle n'indique pas plus une sexualité que les anastomoses si fréquentes du mycélium. Nous reviendrons tout à l'heure sur cette question.

A côté des ascospores, on trouve des spores non formées dans des sporanges, mais souvent aussi réunies en conceptacles. Les pycnides sont des fruits très analogues aux périthèces, seulement, au lieu d'asques, leur surface intérieure est couverte d'un hymenium composé de cellules plus ou moins courbées, fusiformes, dont chacune constitue à son sommet une spore, ordinairement unique; ces cellules, par conséquent, peuvent être considérées comme des basides.

Il se forme ainsi de grandes spores, dites macrostylospores ou stylo. spores, uni ou pluricellulaires. Les pycnides n'existent pas d'une façon aussi générale que les fruits à asques; beaucoup de genres en manquent (Cucurbitaria, Leptosphaeria); quelquefois on ne les trouve que dans certaines espèces d'un genre (Pleospora). Leul' développement, jusqu'à la formation des asques, est analogue à celui des périthèces; cependant elles peuvent aussi dériver d'un véritable tissu parenchymateux (Cicinnobolus, Pycnis); voici comment M. Brefeld a décrit ce développement pour le Pycnis sclerodivora, parasite sur les sclérotes de Peziza sclerotiorum : Les principaux filaments du mycélium se renflent par places jusqu'à devenir deux à quatre fois plus larges; ces renflements sont découpés par de nombreuses cloisons transversales en une série de cellules discoïdes d'vù émanent des branches latérales rayonnantes, serrées les unes contre les autres; puis apparaissent à l'intérieur du filament élargi, des cloisons perpendiculaires ou obliques aux transversales, et il se forme ainsi un véritable tissu parenchymateux; le nombre des branches périphériques augmente, et elles forment comme un chevelu autour du noyau cellulaire central ; elles peuvent se souder, s'anastomoser entre elles, et si on ne suivait le développement, on pourrait quelquefois considérer ces anastamoses comme étant l'origine de toute la formation. Le noyau devient sphérique et a l'apparence d'une gale placée sur le mycélium; il constitue alors une masse de tissu compacte; ses couches extérieures secuticularisent, deviennent noires. Une tache claire arrondie apparaît à son sommet; c'est un orifice entouré de courtes 
hyphes rayonnantes, qui aboutit à une cavité intérieure sans doute formée par suite de l'accroissement du noyau. Le revètement interne de cetie cavité est constitué par des cellules dont chacune, par refoulemsnt (ou bourgeonnement), forme deux à trois spores d'un vert foncé, non supportées par des stérigmates. La cellule se gélifie ensuite; mais de nouvelles spores sont alors formées par la couche sous-jacente, et le processus ne respecte firalement que la paroi extérieure cuticularisée; les cellules placées près de l'ostiole ne forment point de spores, mais constituent par leur allongement les hyphes mentionnées plus haut. Le mucilage se gonfle au fur et à mesure et déborde par l'ostiole en entraînant les spores qui germent de suite. Les pycnides se forment souvent sur des filaments très rapprochés; si ceux-ci arrivaient à se toucher, ils pourraient s'anastomoser, sans doute, comme tous les filaments mycéliens, et alors on serait tenté de regarder le développement des pycnides comme le résultat d'une fécondation. Quand les noyaux sont ainsi rapprochés, ils peuvent se souder par leurs bords, ou même se confondre en une pycnide unique.

Les champignons à pycnides feuvent être parasites d'autres champignons, et il est arrivé que l'on ait considéré alors la pycnide comme appartenant au cycle de développement de l'hôte.

La structure des spermogonies est analogue à celle des pycnides; la surface interne de ces conceptacles consiste encore en un hymenium composé de basides, lesquelles donnent naissance à des spores très petites, ordinairement allongées en bâtonnets, plus rarement rondes ou ovales; ce sont les spermaties. On a souvent considéré ces spermaties comme jouant le rôle d'organe mâle dans la copulation qui précède le développement des pérıthèces. Mais M. Cornu a fait voir que les spermaties des Ascoinycètes peuvent germer, ce qui n'est pas fait pour confirmer leur rôle d'anthérozoïdes. Les spermaties, dont les parois sont minces, sont « les agents de dissémination à grande distance; elles sont très nombreuses, très petites; leur masse semble avoir été allégée de la réserve de nourriture que contiennent les autres spores. Pour leur premier développement elles ne s'accroissent que lorsqu'elles sont arriveees sur le substratum qui leur convient; là seulement elles germent et y demeurent » (M. Cornu). Les stylospores, 
au contraire, paraissent en général munies d'une double enveloppe dont l'externe doit être rompue pour le développement; elles sont acrogènes et chlamydées; elles germent dans l'eau.

Les conidics sont des spores qui ne se forment pas dans des conceptacles, mais à l'extrémité de filaments ; ceux-ci peuvent donuer naissánce à la spore par étrangl̉ement au-dessous du sommet, et de pareils filaments sont tantôt isolés, tantôt réunis par groupes; d'autres fois ils forment des ramifications dichotomiques ou fasciculées et les spores naissent à l'extrémité de toutes les branches. Il arrive encore que l'extrémité d'un filament se renfle en une tête qui supporte de nombreux stérigmates rayonnants avec conidies à l'extrémité de ceux-ci. Souvent plusieurs de ces spores se forment successivement à l'extrémité d'un même rameau ; elles peuvent alors rester réunies en chapelet ou se détacher à mesure de leur formation; d'autres fois elles ne tombent qu'en grand nombre et accolée par un mucilage. M. Cornu a fait voir qu'en réalité il y a deux espèces de conidies dans les Ascomycètes, les unes, homologues des spermaties, les autres des stylospores; il en résulte une grande simplification dans les organes reproducteurs des Ascomycètes; en dehors des asques, il n'y a plus que deux espèces de spores : des stylospores à paroi mince et très petites. Les unes et les autres peuvent naître sur des supports simples ou plus ou moins compliqués, ou être renfermés dans des conceptacles. Sans doute cette division n'est pas absolument tranchée, et existe-t-il des formes intermédiaires; d'un autre còté, on n'a pas réussi toujours à faire germer les spermaties; il en est même de très rudimentaires, et peut-être sont-ce là des spores en voie de régression:

Il y a des cas où les conidies ne germent point, mais où leurs stérigmates et les filaments qui les portent sont susceptibles de produire un nouveau mycélium (Pezizá tuberosa).

Toutes ces formes d'organes reproducteurs peuvent se trouver réunies dans un même cycle de développement; ce n'est pas le cas le plus fréquent. Souvent le mycélium forme d'abord pendant longtemps des conidies, puis surviennent progressivement des périthèces, pendant que les conidies disparaissent; souvent́ aussi (Pyrénomycètes, Discomycètes) la production de conidies se trouve arrêtée par la formation 
de sçlérotes; ceux-ci ne sont en somme qu'un mycélium modifié, une accumulation durable de matière nutritive; placés dans des conditions favorables, ils produisent des périthèces portés par des supports fructifères ; ce développement peut avoir lieu immédiatement ou après un temps de repos plus ou moins long (jusqu'à plusieurs années).

\section{Formation du fruit.}

Gymnoascées. - Le cas le plus simple semble celui décrit récemment par M. Eidam : Sur un filament mycélien, des deux côtés d'une cloison, naissent deux branches qui s'enroulent ensemble en spirale; elles s'affrontent par leur sommet où la cloison commune se trouve résorbée; il y a fusion des protoplasmes et formation d'une cloison dans chaque filament près du sommet; la nouvelle cellule ainsi délimitée donne un asque à huit spores. (Eremascus, g. nov.) D'autres fois, dans la même famille, deux branches semblables naissent ensemble sans fusionner, ou la branche stérile apparait la première, et la branche fertile la contourne en spirale; enfin cette dernière peut aussi s'enrouler autour du filament qui lui donne naissance ou autour d'un filament voisin; il semble donc assez probable que la branche stérile est ici un simple support, que la fusion n'est nullement nécessaire. Dans tous les cas, la spire de la branche fertile se découpe en cellules peu nombreuses d'où émergent les rameaux sur lesquels se formeront les asques ; ceux-ci sont ordinairement géminés; leur support ramifié se lignifie ensuite; le fruit ainsi formé est dépourvu d'enveloppe, d'où le nom du groupe. (MM. Eidam, Baranetzky.)

Périsporiacées. - Les asques, sans paraphyses, sont rẻunis dans un périthèce fermé dont l'enveloppe ne s'ouvre jamais par un pore apical.

C'est à l'entrecroisement de deux filaments que se forme le fruit; le filament inférieur émet ordinairement l'ascogone (ou carpogone), le supérieur le pollinode ou filament mâle (?). Ces deux branches dressées se séparent par des cloisons, le pollinode se divise encore 
une fois, et sa petite cellule terminale vient s'appliquer au sommet de la branche femelle renflée; puis, du filament qui a produit le pollinode, partent des branches qui viennent former l'écorce du fruit; l'ascogone se divise en cellules d'où émanent plus tard les asques. (Erysiphe) (Tulasne, M. de Bary.)

Dans le cas précédent on distingue dès l'origine la branche fertile de la branche mâle qui n'est peut être que le premier filament de l'écorce. Dans le Ryparobius myriosporus on voit apparaître plusieurs branches latérales semblables qui s'enroulent en une petite pelote, puis, à l'intérieur de celle-ci, se différencie une cellule plus grosse qui augmente de volume, ainsi que toute la petite masse; cette cellule produira les asques. Les deux parties, fertile et stérile, ne se distinguent donc pas ici dès la première ébauche (M. Brefeld).

Deux branches s'échappent simultanément du tour de spire inférieur de l'ascogone contourné des Eurotium (M. de Bary); une seule se soude au sommet de l'ascogone; elle ne diffère point de l'autre, ni des branches qui viennent ultérieurement s'appliquer étroitement, comme les premières, sur la spirale qu'elles enveloppent complètement. Toute la formation s'accroit ; les filaments de l'écorce se divisent en cellules qui, proliférant vers l'intérieur, donnent encore naissance au tissu de remplissage. L'ascogone aussi se segmente et de ses articles partent des branches ramifiées qui pénètrent dans le tissu de remplissage et dont les cellules terminales se changent en asques; ceux-ci résorbent tout le tissu et sont à leur tour résorbés par leurs spores qui ne se trouvent plus enveloppées, en dernier lieu, que par un enduit résineux commun. Celui-ci étant très fragile, les spores deviennent libres facilement, et, comme dans les cas précédents, elles rompent, à la germination, leur exospore pour produire un mycélium.

Pyrénomycètes. - Suivant que les conceptacles ont la forme de sphères ou de bouteilles, elles s'ouvrent à l'extérieur par un ostiole ou par un court canal. Les asques sont entremêlés de paraphyses. Les périthèces peuvent être simples, c'est-à-dire isolément implantés sur le mycélium, ou composés, c'est-à-dire réunis plusieurs sur un réceptacle commun (stroma). Dans le premier cas les couches exté- 
rieures de l'enveloppe sont ordinairement dures et de couleur foncée; dans le second cas, c'est la périphérie du stroma qui subit une semblable modification.

Le nuycélium du Claviceps purpurea (M. Tulasne) produit des conidies dont la germination donne d'abord un nouveau mycélium soit directement, soit par l'intermédiaire de conidies secondaires; puis ses hyphes forment un sclérote, le seigle ergoté, sur lequel apparaissent, dans des conditions favorables, les réceptacles fructifères. Des groupes de i:ellules situées sous l'écorce de l'ergot, s'allongent pour cela en filaments qui se réunissent étroitement en un faisceau; celui-ci soulève, puis perfure les couches périphériques. Tous les filaments du faisceau sont égaux; on ne distingue aucune ébauche d'ascogone, et la différenciation en tissus fertile et stérile ne se manifeste que quand déjà les périthèces ont commencé à se former; si l'on coupe une jeune tête, son support produit un ou plusieur's nouveaux réceptacles fructifères. Les asques èt leurs spores unt une forme très allongée.

Dans le Pleospora herbarum le développement a lieu sans ascogone ui pollinode (M. Banke), comme dans le cas précédent.

Dans les Melanospora, Sordaria, Chaetomium, il se forme, au contraire, des ascogones en spirale, mais on n'a pu observer de copulation. Les phénomènes ont été décrits pour ce dernier genre par M. Van Tieghem: une branche s'enroule en spirale; de sa partie inférieure part un rameau plus grêle qui rampe sur la pelote et se ramifie avant d'avoir atteint le sommet; il enlace ainsi le carpogone d'une enveloppe. Enveloppe et carpogone se découpent en cellules et se développent indépendamment l'un de l'autre constituant ainsi le périthèce.

Discomycètes (MM. Tulasne, de Bary, Woronine, Janczewski). Le périthèce est ouvert largement dès l'origine ou au moins à la mafurité ; il se transforme donc en cupule. Les paraphyses peuvent exister ou manquer. Souvent il y a des sclérotes; leur développement peut être différent suivant les circonstances; ceux de Peziza Fuckeliana donnent des conidies, s'ils germent sur le sol, et, s'ils sont enfouis, des cupules pédicellées qui viennent s'épanouir à l'air. Fréquemment les conidies ne germent pas (Peziza tuberosa); elles peuvent même devenir rudimentaires ( $P e z i z a$ sclerotiorum) ou disparaitre Rietsch. 
tout à fait. Souvent les spermogonies précèdent ou accompagnent les périthèces (Tympanis, Rhytisma, etc.); des basides à spermaties peuvent exister dans certaines cupules à asques où elles semblent remplacer les paraphyses (Peziza benesuada) (M. Tulasne). Enfin, on trouve quelquefois dans les cupules deux espèces d'asques; les uns avec huit grandes spores qui donnent directement un mycélium ordinaire ; les autres avec de nombreuses petites spores qui donnent un promycélium avec sporidies (Peziza Durieuana).

Dans les Pezizes, l'ascogone ne se contourne pas en spirale, mais se renfle en vésicule surmontée d'un prolongement recourbé qu'on a considéré comme un trichogyne ; l'ensemble constitue le scolécite; le pollinode vient se mettre en contact avec letrichogyne (Peziza confluens); le trichogyne peut manquer $(P$. granulosa). Dans l'A scobolus pulcherrimus le sclolécite esî une rangée de larges cellules; des rameaux issus des filaments voisins viennent appliquer leur cellule terminale contre la partie supérieure du scolécite, puis d'autres rameaux viennent compléter l'enveloppe. M. Janczervski a constaté que, dans l'Ascobolus furfuraceus, le tissu de la coupe fruciifère et les paraphyses proviennent des filaments corticaux, tandis qu'une cellule unique, la moyenne du scolécite, engendre les hyphes qui constituent par leurs ramifications la couche sous-hyméniale d'où proviennent les asques.

Le sclérote de $P_{e z i z a}$ sclerotiorum donne naissance à un faisceau de hyphes étroitement juxtaposées se terminant presque toutes à la même hauteur; puis l'accroissement s'arrête au centre du faisceau et continue à la périphérie ; en même temps tous ces filaments se ramifient; ainsi se constitue la coupe. Tous les rameaux sont d'abord minces; puis d'autres plus larges, lesfutures asques, apparaissent, s'insinuent entre les premiers qui formeront les paraphyses. Les asques sont produits dans la cupule en direction centrifuge; ceux qui se sont vidés, se trouvent aussitôt remplacés par des asques nouveaux; plusieurs têtes peuvent naître d'un seul sclérote. Asques et paraphyses proviennent donc ici des mêmes hyphes et les différences avec les cas précédents semblent se réduire à une différenciation plus ou moins précoce du tissu en filaments fertiles et stériles.

En résumé, dans toutes les subdivisions précédentes des Ascomy- 
cètes le fruit se forme souvent sans aucune espèce de copulation; dans d'autres cas, des filaments viennent s'affronter l'un contre l'autre, sans qu'il ait été possible de constater une fusion de leurs protoplasmes. Enfin, dans quelques cas rares, on a observé une copulation; mais il n'est nullement démontré qu'elle soit nécessaire au développement ultérieur; elle pourrait être accidentelle ou végétative, et cela est d'autant plus probable que les fruits se ressemblent toujours, qu'il y ait eu ou non fécondation.

Tubéracées. - Le fruit plus compliqué présente une enveloppe extérieure, le péridium, lisse ou échinée, ì couches dures et résistantes en dehors, molles en dedans, sans limite tranchée entre les deux tissus. Le tissu intérieur se prolonge par des cloisons proéminentes plus ou moins compliquées qui divisent la cavité du fruit en compartiment ou loges ; ces loges sont tapissées par des hyphes lâchement entrelacées qui portent les asques. Les spores ne deviennent libres que par la destruction du fruit; le développement de celui-ci n'est connu que dans le Penicillium glaucum, remarquable par ses conidies disposées en chapelets sur un support ramifié en forme de pinceau. Ce développement ressemble d'abord à celui de l'Eurotium ; mais le périthèce se change ensuite en un sclérote qui peut être desséché pendant plusieul's mois ; en les plaçant alors sur un support humide, les hyphes fertiles, par une espèce de germination, émettent des branches ascogènes cloisonnées qui résorbent le tissu de remplissage; sur ces branches les ascosperes se forment en direction basipète; elles sont rangées en. chapelets comme les conidies.

\section{LICHENS.}

(Tulasne, MM. de Bary, Nylander, Schwendener, Bornct, Famintzin, Baranetzler, Rees, Treub, Stabl, Krabbe).

Les Lichens sont des champignons ascomycètes associés à diverses algues; ces algues sont des Chlorophycées ou des Cyanophycées, appartenant à diverses familles de ces deux groupes. On a fait, pour ainsi dire, la synthèse des Lichens (MM. Bornet, Stahl), et en pla- 
çant à la portée d'une spore en germination des gonidies provenant du même Lichen, ou même d'un Lichen différent, on a vu se constituer une nouvelle association. Les filaments du champignon s'appliquent étroitement à la cellule verte que, par leurs ramifications, ils ne tardent pas à envelopper complètement. Les observations ont surtout été concluantes sur les Lichens possédant des gonidies hyınéniales (Endocarpon pusillum, M. Stahl), dont les grandes spores sont projetées en même temps que des gonidies; les filaments issus des spores enveloppent ces gonidies qui augmentent de volume et prennent une couleur vert foncé, en ne se multipliant que lenternent; tandis que les gonidies qui sont restées libres, conservent leur couleur vert pâle et continuent à se segmenter activement; cette association est la première ébauche d'un nouveau thalle qui au bout de quatre à six semaines porte des spermogonies. On connaît des Lichens, et c'est la grande majorité, dont le champignon ne peut vivre que parasite de l'Algue; chez un petit nombre (Arthonia, Graphis) le champignon est tout d'abord saprophyte; ce n'est que plus tard que les gonidies apparaissent et qu'il devient Lichen. Enfin, il y a des formes rangées par les Lichenologues dans les genres de Lichens Artonia, Arthopyrenia, qui sont pendant toute leur vie de véritables champignons saprophytes.

M. Bornet a fait voir que toute gonidie de Lichen peut être ramenée à une espèce d'algue; ses cellules sont plus ou moins altérées dans l'association; mais elles peuvent, néanmoins, s'y multiplier, surtout à la périphérie; d'autres fois, au contraire, la végétation de l'algue se trouve activée. Un même lichen peut contenir des gonidies provenant d'algues différentes, et les mêmes gonidies peuvent se rencontrer sur des Lichens différents. Un séjour prolongé dans l'eau tue le champignon, sans nuire à l'algue. MM. Famintzin et Baranetzky ont vu une algue, le Cystococcus humicola, extraite de divers Lichens, se reproduire par zoospores; M. Woronin a fait des observations analogues sur les gonidies de divers Parmelia.

Quant aux champignons de l'association ce sont presque toujours des Ascomycètes. Tout récemment, M. Oreste Mattirolo a montré, il est vrai, que dans le lichen Cora, le parasite était un Basidiomycète 
voisin des Auriculariees; chaque baside ne produisait qu'une seule spore. Néanmoins l'immense majorité de ces parasites appartient aux Ascomycètes. Dans leur cycle de développement, on trouve encore des pycnides avec des stylospores. Une apparition beaucoup plus fréquente est celle des spermaties (M. Tulasne); elles se forment sur des supports simples ou ramifiés, souvent dans des conceptacles particuliers, les spermogonies, lesquelles précèdent ordinairement la formation des asques. Chez les Ephèbe, les spermogonies se forment sur des individus spéciaux, dépourvus d'asques. M. Cornu n'a pas pu faire germer les spermaties des Lichens.

Mais les spures les plus répandues chez les Lichens, sont les ascospores; elles sont produites par des asques réunis dans des conceptacles en tout semblables à ceux des Dyscomycètes et des Pyrénomycètes. Ces conceptacles, appelés ici apothécies ont, en effet, souvent la forme de cupules ou de disques, mais quelquefois aussi celle de bouteilles ou de sphères, ne s'uuvrant que par un ostiole; dans le premier cas, les Lichens sont dits gymnocarpes, dans le second angiocarpes. Ce fruit appartient au champignon seul; les gonidies ne se trouvent que dans les parties secondaires de l'a pothécie, le pédicelle et le rebord cupuliforme; dans certains Lichens on trouve cependant aussi des gonidies hyméniales (M. Nylander).

La formation des apothécies est endogène, à l'exception des Cœnogonium. La première trace apparait au-dessous de la couche corticale du thalle ou même plus profondément; il se forme une petite pelote arrondie de filaments enchevêtrés; sur la face tournée vers l'extérieur, se produit une forêt de minces paraphyses, entourée, en bas et sur les côtés, par une couche spéciale l'excipulum. Celui-ci s'accroît en surface et forme de nouvelles paraphyses qui s'insinuent entre les anciennes; leur production s'arrête de bonne heure au centre, mais se continue longtemps à la périphérie. Dans la jeune pelote, entre les premières bases des paraphyses, se forment des filaments plus épais, plus gorgés de plasma, ramifiẻs, entrelacés et non cloisonnés. Les extrémités de certaines branches de ces filaments se dressent, s'insinuent entre les paraphyses et constituent les asques; les filaments eux-mêmes restent confinés dans une couche parallèle à la surface 
extérieure, la couche sous-hyméniale; clle apparaît d'abord au centre, puis s'accroît vers la périphérie, où elle continue à produire des asques; ses filaments forment un système distinct de celui des autres filaments du thalle (MM. Schwendener, Fuisting); cn somme, les deux tissus, fertile et stérile, se différencient ici de très bonne heure; néanmoins, le premier rudiment de l'apothécie est neutre ; c'est avec la formation du fruit de Ryparobius que l'analogie est la plus grande. Au-dessous de la couche sous-hyméniale, s'en trouve une autre, l'hypothécie, souvent puissante et donnant naissance aux paraphyses; à l'état adulte, elle se distingue à peine de l'excipulum. En grandissant, l'apothécie se voûte et perce la couche du thalle qui la recouvre; elle peut rester sessile, l'hyménium et l'excipulum périphérique paraissant seuls au dehors, ou bien être soulevée sur une proéminence du tissu, ou bien être portée sur un pédicelle.

Ce n'est pas là l'unique mode de formation de l'apothécie ; récemment M. Stahl en a décrit un autre qui rapproche encore davantage les Lichens des Ascomycètes, et qui démontre que, dans ce groupe aussi, la distinction des deux tissus fertile et stérile peut s'établir dès la première ébauche. Sur le thalle de Collema microphyllum, on peut trouver des apothécies à tous les états de développement. Un filament de ce thalle, qui ne se distingue en rien des autres, emet une hyphe plus grosse, d'épaisseur uniforme; la partie inférieure de l'hyphe est cnroulée en spirale et composée en moyenne de douze cellules; sa partie terminale, faisant suite à la première, compte à peu près autant d'éléments, se dirige en ligne droite ou courbe vers la surface du thalle, et proémine au dehors par sa pointe. Il y a donc un ascogone surmonté d'un trichogyne; souvent toute la surface du thalle se montre couverte de ces pointes de trichogyne. L'apparition des ascogones est favorisée par un temps pluvieux qui facilite aussi la sortie des spermaties de leurs spermogonies; celles-ci se trouvent tantôt sur le même thalle, tantôt sur un thalle différent; l'arrivée de l'eau amène leur déhiscence et l'expulsion des spermaties englobées dans une goutte de mucilage. M. Stahl trouva souvent des pointes de trichogyne avec plusieurs spermaties adhérentes et accolées; it en remarqua un relié au trichogyne par un court prolongement cellulaire 
ou pont de copulation, et les cavités des deux cellules paraissaient alors en communication. Les ascogones plus avancés étaient munis d'une enveloppe corticale, formée par les hyphes voisines du thalle, et non par l'ascogone luj-même; souvent des chapelets de gonidies se trouvent englobés dans la même enveloppe, mais ils ne tardent pas a être résorbés. A la surface de la pelote, les filaments corticaux forment la forêt de paraphyses dirigées vers la surface du thalle; les cellules de l'ascogone se multiplient; puis le développement continue d'une façon analogue au cas précédent, les deux systèmes d'hyphes restant rigoureusement distincts. L'ascogone forme la couche soushyméniale; les hyphes qui la constituent, donnent naissance, par refoulement, aux asques, lesquels s'insinuent entre les paraphyses. La pointe extérie ure du trichogyne se flétrit ; ses cellules internes épaississent leurs parois et font quelquefois encore reconnaître le trichogyne dans les jeunes apothécies. M. Stahl étendit ses observations à d'autres genres, qui donnèrent les mêmes résultats (Physma, Leptogium, Parmelia) :

La présence de ce trichogyne, qui ne prend part ni à la formation des asques, ni à celle du tissu fertile, mais qui vient toujours émerger à la surface extérieure;

Sa surface gluante, très apte à retenir, à fixer les corps étrangers, les spermaties, par exemple, qui viennent, en effet, s'y accoler, et qui fusionnent très probablement avec le trichogyne;

L'épaississement ul térieur des parois trichogyniennes, qui se propage depuis la cellule extérieure jusqu'à celle qui touche à l'ascogone,

Le développement de l'ascogone consécutif seulement à cet épaississement;

Le fait enfin que l'avortement ultérieur des ascogones était corrélatif, dans la plupart des cas, d'un développement incomplet des spermogonies,

Toutes ces circonstances démontrent, d'après M. Stahl, qu'il y a ici véritablement un acte de fécondation.

La fécondation avait été admise aussi d'une façon générale pour les Ascomycètes. M. Van Tieghem, d'un côté, M. Brefeld, de l'autre, firent 
voir que ces phénomènes sont susceptibles d'une tout autre interpré. tation; qu'il n'y a là, sans doute, qu'une simple soudure mycélienne, comme elles sont si fréquentes daus les champignons en général. Leur opinion fut bientôt partagée par un grand nombre de botanistes.

Il est certain qu'aujourd'hui la découverte de M. Stahl est Je plus solide argument en faveur de la sexualité des Ascomycètes, et ce n'est pas dans la fusion, si fusion il y a, de la spermatie avec la cellule apicale du trychogyne, que gît la difficulté, ni dans l'ascogone analogue à celui des Eurotium, Peziza, etc.; c'est dans le trichogyne, lui-même, reliant ensemble la spermatie et l'ascogone, et c'est, en effet, principalement sur ce point que M. Stahl a insisté.

A moins donc que ce trichogyne ne reçoive une autre interprétation mieux fondée, il est assez difficile de ne pas admettre la sexualité pour le champignon des Collema. Mais ce phénomène n'est pas général; les recherches citées plus hant, celles toutes récentes de M. Krabbe, montrent, en effet, que les apothécies peuvent náitre sans fécondation, et même sans ascogone. Dans les Bceomyces, Cladonia, Lecidia, Pertusaria, Phlyctis, Phialopsis, il n'y a, dans le développement du fruit, aucun indice de sexualité ; les tissus fertile et sterile se différencient de bonne heure, il est vrai, dans l'ébauche apothécienne, sous forme de filaments dissemblables ; mais cette ébauche ellemême, antérieure à la différenciation des hyphes, n'est pas autre chose qu'un bourgeonnement du tissu du thalle. M. Krabbe a montré encore que les apothécies se multiplient abondamment par scissiparité et par bourgeonnement.

Si donc il y a sexualité dans les Lichens, ou plus généralement dans les Ascomycètes, elle n'est point générale ; les fruits, développés à la suite de la fécondation, ne diffèrent en rien de ceux qui, dans les mêmes groupes, se forment par un simple bỏurgeonnement, et l'acte lui-même de la fécondation affecte, en général, une autre allure que dans les végétaux pourvus de chlorophylle. On ne peut donc attribuer à la sexualité l'importance qu'elle possède ailleurs, et il semble bien difficile de ne pas admettre, dans les champignons, une dégradation consécutive du parasitisme, non seulement dans les organes sexuels et dans les fruits qu'ils produisent, mais encore dans tous les organes 


\section{$r-105-$}

de propagation, ce qui n'implique en aucune façon une diminution de la faculté reproductrice. C'est en somme sur le degré de cette dégradation que porte toute la discussion.

Les asques ne diffèrent point de ceux des Discomycètes et Pyrénomycètes; leur paroi est souvent épaissie, capable de se gonfler fortement; les spores sont ordinairement au nombre de 8 dans chaque thèque, quelquefois 1 à 2 (Umbilicaria), 2-3 ou 4-6 (Pertusaria), plus de 100 (Bactrospora, etc.); elles peuvent être pluricellulaires. Sous l'influence de l'humidité, les spores sont expulsées avec le liquide qui remplit l'asque; le gonflement des paraphyses semble ètre la cause directe de la déhiscence, par la pression que ces organes exercent alors sur les asques. Pour la germination, l'enveloppe de la spore est rompue, et le contenu, entouré d'une mince membrane, s'allonge en un filament. Les grosses spores unicellulaires de Pertusaria, Megalospora, etc., développent, au contraire, un grand nombre de pareils filaments.

Les sorédies constituent les organes de la propagation végétative; ce sont des gonidies isolées ou des groupes de gonidies, entrelacées étroitement de filaments rnycéliens, qui sont expulsées du thalle et peuvent développer immédiatement un thalle nouveau. Dans l'intérieur de Ja couche gonidienne, on voit une cellule verte se diviser à plusieurs reprises; ses cellules filles sont séparées à mesure de leur formation par des branches mycéliennes qui, se multipliant activement, enveloppent les nouvelles gonidies à mesure qu'elles se formant. Ainsi prennent naissance de très nombreuses sorédies; leur pression finit par déchirer la couche corticale du thalle, et la masse sorédienne n'est plus retenue dans la fente produite que par quelques hyphes; les propagules s'échappent un à un à travers ce réseau. La sorédie peut se développer sur le thalle même (Usnea barbata) et produit alors ce qu'on a appelé une branche sorédiale. 
ECIDIONYYCÈTES.

(MM. Tulasne, de Bary, Rees, Schroeter, Cornu, Oersted).

Les Acidiomycètes semblent se rattacher aux Ascomycètes (M. de Bary); les sporanges de ces derniers, les asques, se seraient réduits ici à un chapelet de spores. Le fruit caractéristique de ce groupe, les écidies, sont en effet des cunceptacles formés par le mycélium à l'intérieur et au-dessous de l'épiderme de la plante mère; ils sont entourés par une couche de filaments mycéliens; ces conceptacles rompent ensemble l'épiderme et s'ouvrent au dehors; ils ont alors la forme d'une coupe dont la paroi, ou le péridium, se compose d'une assise de cellules hexagonales disposées en séries; du fond de la çupe s'élèvent de nombreuses basides serrées et dirigées vers l'orifice de l'écidie; chacune forme, par étranglements successifs, une série de spores d'abord rendues polyédriques pa: leur pression mutuelle; elles s'arrondissent ensuite et s'échappent par l'ouverture de la coupe.

Sur la même plante nourricière se développent antérieurement aux écidies et simultanément aussi, des spermogonies, semblables à celles des Ascomycètes; elles produisent encore des spermaties qui sont susceptibles de germer en dunnant des sporidies ou spores secondaires (M. Cornu). Les spermogonies accompagnent aussi, quoique plus rarement, les autres formes de spores (Uredo et Teleutospores, M. Winter).

Les Ecidiomycètes sont remarquables par leur alternance de générations très nettement marquée, et par leur hétéroécie ou hétéroxénie liée à cette alternance. I.es spores des écidies ne germent pas en effet sur le premier hôte, mais seulement sur un hôte différent; leur tube germinatif pénètre par les stomates et formse un nouveau mycélium dans le tissu nourricier. Sur ce mycélium apparaissent des stylospores, ordinairement rouges, dites aussi urédospores, qui sont une forme particulière de conidies. Ces organes sont destinés à propager le parasite dans le deuxième hôte, sur lequel, en effet, ils germent directe 
ment aussi bien que les écidiospores. Mais ver's la fin de l'été, les urédospores sont remplacées par des spores durables, chronispores ou téleutospores, enveloppées d'une épaisse membrane et ne germant qu'au printemps suivant, après une période de repos. Elles développent alors un court promycélium portant des sporidies que le vent peut emporter au loin et qui germent de nouveau sur le premier hôte, dont elles perforent l'épiderme pour produire à son intérieur, sur un nouveau mycélium, des spermogonies, puis des écidies. La première ébauche de ces dernières consiste en une petite pelote de filaments mycéliens entrelacés et jusqu'à présent on n'a rien observé dans leur développement qui ressemblât à un ascogone (M. de Bay).

Les $A$ Ecidiomycètes ou Urédinées comprennent un très grand nombre d'espèces. Le Puccinia graminis forme ses écidies sur le Berberis vulgaris, ses urédospores sur les Graminées; le Puccinia straminis, écidies sur Lycopsis arvensis, Anchusa officinalis, etc., urédospores sur les Graminées; Puccinia coronata, écidies sur les Rhamnus, urédospores sur Graminées (Avena, Hoḷcus); Gymnosporangium (Podisoma) conicum, écidies sur Sorbus (Roestelia cornuta), urédospores sur Juniperus communis. Ecidium abietinum (Abies excelsa) avec téleutospores sur Rhododendron hirsutum.

Toutes les espèces ne sont pas aussi riches en organes de propagation différents. Dans les Endophyllum, en effet, les écidiospores, directement ou par l'intermédiaire de sporidies, donnent de nouveau un mycélium à écidies. $D^{*}$ un autre côté le Chrysomyxa Abietis ne possède ni écidies, ni urédospores; mais les sporidies provenant des téleutospores forment un nouveau mycélium à téleutospores. Il peut donc $\mathrm{y}$ avoir simplification dans deux sens et réduction à une des deux formes principales d'organes de reproduction : écidiospores ou téleutospores. Les Chrysomyxa par la simplification dans leurs organes reproducteur's conduisent aux Hyménomycètes.

TRÉMELLINÉES.

Le corps fructifère consiste en une masse gélatineuse traversée par de minces filaments ; cette masse n'est que le résultat de la géli- 
fication des parois externes de ces filaments. Toute la surface du corps fructifère est occupée par l'hyménium, formé par les cxtrémités renflées des filaments. Chacun de ces renflements plicés près de la surface se découpe en quatre basides juxta ou superposées et donnant naissance chacune à un stérigmate allongé ; ceux-ci viennent proéminer à la surface du champignon où ils forment chacun une spore. Les spores produisent le mycélium soit directement, soit par l'intermédiaire d'un promycélium portant des faisceaux de sporidies en forme de bâtonnets.

M. Tulasne a décrit en outre des filaments, souvent mêlés aux basides, et produisant des conidies (spermaties); quelquefois ces filaments couvrent exclusivement certaines parties du champignon, surtout les lobes inférieurs, en leur donnant une couleur orangée brillante; on n'a pas réussi à faire germer ces spermaties.

Les Trémellinées peuven entre les Ecidiomycètes et les Basidiosporées.

\section{HYMÉNOMYCÈTES.}

Les Hyménomycètes forment avec les Gastéromycètes les véritables Basidiosporées, auxquels on ajoute quelquefois encore les Urédinées. lls sont caractérisés par la présence des basides; ce ne sont pas là des formations nouvelles, car nous les avons déjà trouvéès, moins nettement caractérisées, il est vrai, dans les Trémellinées et les Ecidiomycètes, et d'une façon plus générale on peut même rattacher les basides à toutes les stylospores. Mais les basidiospores deviennent ici la forme unique des organes de propagation, en même temps le corps végétatif acquiert un développement et une différenciation plus grands que dans toutes les autres divisions.

Les basides sont des cellules, ordinairement allongées, séparées par une cloison du filament qui leur a donné naissance, gorgées de protoplasma finement granuleux et incolore, et munies d'une mince membrane. Au sommet' de la baside se forment, par refoulement de dedans en dehors, de minces branches en forme d'alène, dont l'ébau- 
che coïncide avec la disparition du noyau de la baside ; ce sont les stérigmates, au nombre ordinairement de quatre, quelquefois de deux ou de huit, ou en nombre indéterminé. Quant le stérigmate a atteint une certaine longueur, soll extrémité se renfle en vésicule, laquelle en s'agrandissant devient la spore; presque tout le protoplasma de la baside émigre dans les spores; quand celles-ci ont atteint leur volume définitif, elles se séparent de la baside par une cloison qui ne se forme pas ordinairement au sommet même du stérigmate, de sorte que la baside détachée semble munie souvent d'un court pédicelle.

Les basides, presque toujours entremêlées de paraphyses, constituent l'hyménium qui est librement étalé à l'air dans les Hyménomycètes. La germination des spores issue des basides, donne naissance à un mycélium, rarement parasite, vivant ordinairement dans l'humus ou sur le bois mort; il présente de nombreuses anastomoses entre ses filaments (souvent anastomoses en boucles); fréquemment sur le mycélium on voit naître des sclérotes. C'est sur ceux-ci ou directement sur les filaments mycéliens que se forment les réceptacles fructifères; leur forme est très variée : dans les Auriculariées le réceptacle se réduit à un enduit étalé sur le substratum et ayani sa surface libre recouverte par l'hyménium.

Dans les Clavariées il forme des touffes buissonneuses sur lesquelles s'étend encore extérieurement la couche fertile.

Dans les Agaricinées, Polyporées et Hydnées, le réceptacle prend la forme d'un chapeau sessile ou pedicellé, souvent en forme d'ombrelle; ses bords peuvent se retrousser en haut, ou se recourber davantage en bas, d'où résulte une apparence d'entonnoir dressé (Cantharellus cibarius) ou de cloche (Coprinus).

L'hyménium peut être étalé en couche lisse (Auriculariées et $\mathrm{Cla}$ variées), ou bien, au contraire, recouvrir toutes les proéminences à la face inférieure du chapeau; cess prolongements affectent la forme de dents (Hydnées), de bourrelets, de lames rayonnantes (Agaricus), ou des lames enchevêtrées en réseau à mailles larges (Dcedalea), ou à mailles serrées, constituant des tubes (Boletus, Polyporus). L'hyménium est formé par les cellules terminales des hyphes constituant le chapeau et ses proéminences; il est formé de basides, de paraphyses 
et cystides. Les premières sont isolées ou en groupes, entremêlées de paraphyses qui sont les terminaisons stériles des hyphes, et qui sont moins riches en protoplasma. Les cystides sont des paraphyses plus grandes; elles sont moins nombreuses et eccupent de préférence le rebord libre des lamelles ou bourrelets.

Une cellule quelconque du mycélium ou de la surface du sclérote peut, dans les Coprinus, devenir l'origine d'un réceptacle fructifère; pour cela, par bourgeonnement latéral, elle forme plusieurs minces filaments qui ne tardent pas à se ramifier et à s'entrelacer, constituant une petite pelote dont les éléments sont bientôt plus serrés et plus denses à l'intérieur.

Chaque pelote se compose donc d'une enveloppe corticale de hyphes lâches et d'un noyau ḋense de pseudoparenchyme ; ce noyau, par développement basifuge, constitue le pédicelle qui n'atteint que beaucoup plus tard, et d'une façon brusque, son allongement définitif. Au sommet du noyau il y a une formation abondante d'hyphes nouvelles dont les unes, serrées et se terminant au même niveau, descendent le long du noyau et forment, par leur ensemble, une coupole qui est l'origine du chapeau; les autres se mêlent aux hyphes de la couche corticale du sommet avec lesquelles elles formeront la volva du chapeau. Celle-ci se continue sur les bords avec la large couche externe du pédicelle; l'ensemble de cette enveloppe corticale lâche se transformera en velum universale.

Il n'y a, dans ce développement, aucune trace ni d'ascogone, ni de pollinode (M. Brefeld) et tout se réduit à un simple bourgeonnement végétatif.

Des centaines d'ébauches fructifères peuvent naître sur le même sclérote; mais quelques-unes seulement (une seule sur les petits sclérotes) atteignent leur développement définitif; sur les cultures de mycélium M. Brefeld ne vit jamais plus de vingt ébauches à la fois.

En détachant ces nodules il s'en forme d'autres à la même place; en coupant le sclérote, chaque cellule superficielle intacte du fragment peut encore devenir l'origine d'un réceptacle; en décapitant un jeune réceptacle, il s'en forme un autre sur le moignon du premier. D'un autre côté les réceptacles rudimentaires, ou les chapeaux coupés 
en morceaux, quand on les place dans des liquides nutritifs, se développent en mycélium ordinaire.

Dans les Agaricus le développement des chapeaux est tout à fait analogue à celui des Coprinus; mais plusieur's cellules semblent nécessaires pour constituer la première ébauche. 11 n'y a rien qui ressemble à une fécondation, et le développement des réceptacles fructifères est un simple bourgeonnement végétatif.

Dans les deux genres précédents on rencontre quelquefois sur le mycélium des supports à bâtonnets; ils sont peu ramifiés et leurs cellules (quelquefois réduites à une) produisent sur des prolongements, analogues à des stérigmates, des bâtonnets extrêmement petits qui tombent après s'être séparés par une cloison. Ces bâtonnets sont, en général, incapables de germer et doivent être considérés comme des conidies (spermaties) rudimentaires; ils disparaissent avant que les fruits soient ébauchés.

Quelques Hyménomycètes montrent en outre des conidies, ordinairement sur la face supérieure du chapeau : Fistulina hepatica, Polyporus sulfureus (M. de Leynes), Pleurotus ostreatus (M. Patouillard). MM. Berkeley et Broonie auraient noême constaté des thèques sur un Polyporus.

Mais ce sont là, jusqu'à présent du moins, des formations rares, et la grande majorité de ce groupe se trouve réduite à une seule espèce de spores capables de germer; toute sexualité a disparu.

Ces spores peuvent se développer immédiatement; elles conservent plus d'une année leur faculté germinative (Coprinus, M. Brefeld). Au point diamétralement opposé à l'insertion de la spore sur le stérigmate, un pore étroit de l'enveloppe de la spore donne issue à une vésicule qui s'accroît rapidement et dépasse bientôt le volume de la spore : la vésicule produit alors un ou plusieurs filaments à accroissement terminal; les cellules autres que la terminale ne se divisent pas, mais peuvent chacune donner naissance à une branche. 
GASTÉROMYCÈTES.

Les Gastéromycètes peuvent être considérés comme le type culminant des champignons (M. de Bary). La formation des spores ressemble entièrement à celle des Hyménomycètes; mais leur réceptacle fructifère est toujours angiocarpe et l'hyménium ne se développe que dans les cavités on loges à l'intéricur de ce réceptacle. La structure de celui-ci est très variée; toujours on distingue une paroi externe, le péridium, et un tissu interne, la gleba, coupée par des cloisons qui délimitent les loges; c'est sur ces cloisons que prennent naissance les basides.

Les stérigmates sont très peu développés dans les Scleroderma, Phallus, ete.; dans les Bovista, la cloison de séparation de la spore se forme vers la base du stérigmate qui reste attaché à la spore comme un pédicelle. Les basides de Geaster tunicatus se prolongent en un col conique dont le sommet porte six stérigmates divergents. Ordinairement il" y a 8 spores par basides; mais leur nombre peut être aussi de 2 (Octaviana), 4 (Hymenogaster). Le mode deformation de ce réceptacle n'est pas encore bien connu; d'après les observations faites jusqu'à présent (MM. de Bary, Sorokin) la première ébauche n'est point le résultat a'une fécondation, et consiste en une petite pelote de filaments semblables, courts, entrelacés, ramifiés dichotomiquement.

Les spores à la maturité sont tantôt isolées, tantôt réunies par un mucilage; elles deviennent libres, soit simplement par la rupture du péridium, soit à l'aide de différenciations plus ou moins compliquées des tissus de la gleba.

La germination n'a encore pu être obtenue que pour les spores de Crucibulum. 


\section{MUSCINÉES}

(Mirbel, Hofmeister, Schimper, MM. Leitgeb, Nägeli, Pringshoim, Janczewsli, Kionitz-Gerloff, Berggren, Goebel, etc).

Les Muscinées forment un grand groupe naturel de plantes terrestres, supérieures aux véritables plantes aquatiques, les Algues, non seulement par la plus haute différenciation de leur corps végétatif, mais aussi par le perfectionnement de leurs organes reproducteurs. Cette supériorité qui s'explique suffisamment par la différence de milieu, la station aérienne étant évidemment plus favorable au développement des plantes que la station aquatique, cette supériorité n'est cependant pas telle que nous ne puissions trouver des points de rapprochement entre les Muscinées les plıs inférieures et certaines Algues plus perfectionnées, habitantes des eaux douces.

Dans les Vaucheria, l'oospore donne naissance à une génération asexuée identique à la série de celles qui lui succéderont; dans les Confervacées, la génération agame issue de l'œuf est différente, au contraire, des générations agames subséquentes et consiste en général simplement en un certain nombre de zoospores; dans les Coléochétées, l'œuf se transforme d'abord en un tissu cellulaire dont chaque cellule donne ensuite naissance à une spore. Ce cas est très analogue à celui des Ricciées parmi les Hépatiques; seulement ici le sporogone reste fixé sur la génération sexuée, tandis qu'il se détache du thalle dans les Coléochétées; cependant, même sous ce rapport, ces derniers offrent encore une transition; car leur œuf fécondé adhère quelque temps au thalle et s'y entoure d'une écorce.

D'un autre côté, dans les Floridées, le cystocarpe représente un individu asexué, différent de ceux auxquels il donnera naissance et entièrement parasite de la génération sexuée; il parait homologue du sporogone des Muscinées. L'analogie devient plus grande encore 
dans les Lémanéacées par la suppression de toutes les spores asexuées, à l'exception des carpospores qui résultent directement de la fécondation et par la succession de trois générations différentes : protonema, individu fructifère, cystocarpe, parasites l'une sur l'autre.

La similitude avec certaines Algues est donc très grande, eu égard à la différence des milieux; ce qui, à notre point de vue différencie les deux groupes pris dans leur ensemble, c'est que les Muscinées montrent une alternance régulière de générations, sexuée et asexuée ; c'est que les organes de reproduction atteignent ici un notable perfectionnement, (quoiqu'au fond il n'y ait pas une différence essentielle entre les archégones et les oogones). Sous ces deux rapports les Muscinées se? rapprochent des Cryptogames vasculaires; si les plantes de ces deux groupes sont adaptées à la vie terrestre, leur fécondation ne peut cependant avoir lieu que par l'inter'médiaire de l'eau; elles restent donc encore jusqu'à un certain point sous la dépendance du milieu aquatique et leurs anthérozoïdes conservent leur motilité, tandis que leurs corps reproducteurs agames sont immobiles.

Dans les Muscinées, l'acte sexué a pour résultat la formation d'un individu asexué, déjà beaucouip plus développé et plus différencié que le cystocarpe des Coléochétées et des Floridées, mais encore entièrement parasite néanmoins sur la génération sexuée; aussi est-il bien inférieur à l'individu qui lui correspond dans les Cryptogames vasculaires où il réussit à s'affranchir. Cet individu asexué est appelé ici fruit ou sporogone (M. Sachs); par ses spores agames il reproduit la génération sexuée, non pas directement cependant, mais par l'intermédiaire d'un jroembryon, d'un protonema, qui donne naissance, latéralement ou à son sommet, à la véritable plante, munie d'anthéridies et d'archégones. Des spores asexuées des Algues il n'est donc resté que les carpospores; néanmoins les Muscinées possèdent encore une riche propagation végétative, pius active même et pliss variée pans la classe supérieure de ce groupe, les Mousses, qui sont mieux adaptées à la vie terrestre et qui élèvent davantage au-dessus du sol leur tige portant les organes reproducteurs.

Dans les Hépatiques, le protonema est rudimentaire et le sporo- 
gone, assez simple de structure, reste renfermé jusqu'à la maturité des spores, dans l'archégone (coiffe), déchirée alors par le brusque allongement du pédicelle. Dans les Mousses, le protonema est développé et la coiffe se trouve arrachée de bonne heure par le sporogone plus différencié.

\section{ANTHÉRIDIES.}

Les anthéridies sont des corps cellulaires pédicellés, sphériques ou ellipsol̈daux. Le pédicelle est court, quand l'anthéridie est plongée dans le tissu (Anthoccros, Marchantia), long, quand elle est libre; il est formé de une à quatre séries de cellules. L'assise extérieure du corps de l'an théridie, stérile et riche en chlorophylle constitue la paroi; elle jaunit à la maturité. Les cellules internes petites, nombreuses, serrées, développent chacune un anthérozoïde. La paroi de l'anthéridie se déchire à la maturité au sommet sous l'influence de l'eau qui gonfle les membranes des cellules mères; la déhiscence peut se faire aussi par la chute des cellules du sommet (Fossombronia), ou par une fente (Mousses). Le contenu se vide alors successivement par saccades, quelquefois brusquement par une sorte d'explosion (Frullania); les cellules mères s'isolent dans l'eau et les anthérozoïdes s'en échappent. Ceux-ci sont des filaments enroulés en spirale et formant une à trois spires; leur extrémité postérieure est renflée; l'antérieure, finement effilée, porte deux longs cils grêles dont les battements provoquent les mouvements des anthérozoïdes; ils portent ordinairement à leur extrémité postérieure une petite vésicule délicate. Leur développement est analogue à cel̄ui des Chara décrit par M. Schmitz (M. Gobel); le noyau de la cellule mère forme directement le corps de l'anthérozoïde en condensant sa couche périphérique qui se découpe en une bande spiralée; en même temps la partie centrale du noyau devient moins dense et se change en vésicule; l'extrémité antérieure et les cils qu'elle porte, proviennent seuls du protoplasma de la cellule.

Dans les Hépatiques le mode de développement de l'anthéridie est différent suivant les genres; elle naît toujours cependant de la papille proéminente d'une cəllule qui est superficielle, excepté dans les Antho- 
ccros. La papille se sépare par une cloison transverse, puis se partage en une cellule inférieure qui formera le pédicelle et une cellule supérieure ou corps de l'anthéridie.

Dans les Mousses la cellule mère de l'anthéridie peut être une cellule terminale (Fontinalis, M. Leitgeb) qui tout à coup transforme sa segmentation trisériée en une segmentation bisériée; les deux séries de segments se partagent ensuite par des cloisons tangentielles, de sorte que chaque section transversale présente alors deux cellules externes formant la paroi et deux internes, origine des cellules mères. Dans les Andraea, la cellule mère primitive sépare d'abord par deux cloisons transversales successives la cellule du support, puis celle du pédicelle; elle se comporte ensuite comme dans les Fontinalis. Dans Sphagnum, il se forme un plus grand nombre de cloisons transversales, qui se divisent ensuite en croix pour constituer le pédicelle; puis la papille terminale se renfle et se divise irrégulièrement par des cloisons obliques.

ARCHÉGONES.

L'archégone, avant la fécondation, est une sorte de bouteille dont le ventre renflé est inséré sur une base étroite et se prolonge supérieurement en un long col. La cellule centrale est renfermée dans le ventre; elle est surmontée d'une rangée de cellules centrales du col, rangée qui s'arrête au-dessous de la dernière assise du col ou opercule.

L'archégone procède toujours d'une ceillule centrale superficielle (elle est plongée dans le thalle chez les Anthoceros). Cette cellule, proéminente en forme de papille, se cloisonne transversalement, et se transforme ensuite directement en archégone (Riccia seulement), ou sépare d'abord à sa base la cellule destinée à former le pédicelle; de plus, dans les Mousses elle se segmente encore deux fois inférieurement et les deux cellules qui en résultent, prennent ultérieurement une part active à la formation du ventre de l'archégone.

La papille terminale, ou cellule mère de l'archégone, se partage alors par trois cloisons longitudinales, deux anticlines et une péri- 
cline(1), en une cellule médiane et en trois cellules périphériques; chacune de ces dernières se divise ensuite en deux cellules juxtaposées par une cloison longitudinale radiale; la cellule médiane se divise transversalement vers son sommet en une cellule operculaire et une cellule interne qui est la cellule mère de la rangée axile. Au-dessous de l'operculaire se trouvent donc placées alors sept cellules longitudinales dont une centrale et six périphériques (dans les Jungermanniées il n'y a que cinq cellules périphériques); elles se divisent toutes en deux par une cloison transversale et forment ainsi deux étages ; l'étage supérieur donne naissance au col, l'étage inférieur au ventre de l'archégone.

Par des segmentations transversales les six cellules périphériques du col se tranforment en six rangées cellulaires, tandis que la cellule centrale ou cellule du canal du col se divise en une série axile, composée finalement de 4, 8, 16 cellules et même davantage dans les Mousses. Il y a, en effet, entre les deux classes une différence importante dans le rôle de la cellule operculaire; elle ne prend aucune part à la formation du col dans les Hépatiques; elle le ferme seulement supérieurement et se partage d'abord en croix, puis les quatre cellules ainsi formées se segmentent encore radialement (Riccia) ou parallilement aux premières cloisons cruciales (Jungermanniées). Dans les Mousses, au contraire, la cellule operculaire reste active et contribue à allonger le col ; pour cela elle se segmente d'abord, comme la papille initiale par trois cloisons longitudinales, deux anticlines et une péricline, puis par une transversale; il se forme ainsi une nouvelle operculaire et un nouvel étage à trois cellules périphériques et une axile. Le même processus se répète 5 ou 6 fois, ou même plus dans les Andraea; puis cette activité s'arrête et la dernière operculaire se divise par deux cloisons en croix.

L'étage inférieur de la papille initiale forme le ventre de l'archégone; sa cellule axile ou cellule centrale ne se divise qu'une seule fois par une cloison convexe en bas et donne ainsi une cellule supérieure ou cellule ventrale du canal et une cellule inférieure ou cellule em-

(1) Cloisons courbes tontes deux; les périclines sont parallèles à la paroi de la cellule mère, les anticlines viennent s'insérer perpendiculairement sur cette paroi (M. Sachs). 용 
bryonale. Les six cellules périphériques se segmentent radialement et transversalement pour donner naissance à la paroi du ventre qui suit l'accroissement de la cellule embryonale; elles forment donc des rangées plus nombreuses que dans le col (jusqu'à 24 Preissia); de plus, dans les Mousses les mêmes cellules prennent des cloisons tangentielles, de sorte que la paroi ventrale de l'archégone se compose de deux (Bryacées, Phascacées, ou quatre assises (Sphaignes).

Les membranes transversales des cellules de la rangée axile se résorbent, excepté celle qui sépare la cellule embryonale de la cellule ventrale du col; cette dernière se transforme en mucilage, ainsi que les parois longitudinales des cellules du canal; le protoplasma de ces cellules est comprimé en un mince cordon. Le mucilage exerce aussi une pression contre les cellules operculaires; il finit par les écarter et par l'orifice ainsi formé le cordon protoplasmique est d'abord expulsé; le mucilage s'accumule ensuite à l'orifice du col et arrête les anthérozoïdes qui pénètrent alors par le col ịusqu'à la celluile embryonale; à ce moment le protoplasma de celle-ci s'est contracté en oosphère et se présente nu aux anthérozoïdes. Le noyau de l'oosphère est alors central (M. Strasburger) et sa région supérieure plus claire constitue la tache réceptrice, par laquelle pénètre l'anthérozoïde; il se fusionne avec l'œuf qui ne tarde pas à s'entourer d'une membrane.

La fécondation ne peut avoir lieu que par l'intermédiaire de l'eau; il est probable que souvent les gouttelettes d'eau entraînent les anthérozoïdes dans le voisinage des archégones, quand les Muscinées croissent sur des nıurs, sur des arbres. On a constaté que dans les Frullania, les plantes mâles sont souvent placées plus haut sur l'arbre que les plantes femelles. On a trouvé aussi de petits insectes, des mites, avec une gouttelette de la bouillie anthéridienne; la fécondation doit donc s'opérer aussi par ces auxiliaires. Dans les Mousses elle est souvent facilitée par la réunion daus une même fleur des plantes mâles et femelles; elle l'est d'une façon générale par les faibles dimensions des organes sexuels et par le peu d'élévation de leurs supports. Néanmoins, la propagation végétative possède ici, une très grande importance; certaines Mousses ne fructifient que rarement et n'en couvrent pas moins d'un épais gazon de grandes étendues de terrain; il en est même qui, 
dans certaines contrées, ne se multiplient que par cette voie et dont il n'existe alors que des individus femelles (Lumularia vulgaris).

Mode de distribution des organes sexuels.

Hépatiques. - Les organes sexuels se forment en direction acropète. Dans les Hépatiques à thalle, c'est sur la face supérieure éclairée de celui-ci qu'ils apparaissent, dans le voisinage de la nervure médiane ou sur cette nervure, quand il y en a une. Les individus peuvent être monoïques ou dioïques; dans ce dernier cas les mâles sont ordinairement de moindre taille (Sphaerocarpus terrestris); des branches mâles et femelles sont souvent aussi réunies sur un même thalle. Les organes sexuels peuvent être répartis irrégulièrement sur une branche ordinaire du thalle; mass plus souvent certaines parties d'un branche ou une branche entière se transforment d'une façon spéciale et constituent des inflorescences; les organes sexuels sont alors rassemblés en petits groupes près du sommet de la branche (Targionia), ou en groupes plus grands, enfoncés dans le tissu qui s'élève dans toute la région fertile, au-dessus du thalle en une proéminence portant tout le groupe (Boschia); au milieu de ce disque proéminent, le tissu peut prolıférer et former une saillie axile, sur les flancs de laquelle les archégones apparaîtront nichés dans de petites excavations (Corsinia). Ces disques peuvent alors être soulevés au-dessus du thalle par des pédoncules plus ou moins élevés (Plagiochasma). Mais le sommet même de la branche se trouve finalement compris dans ces formations (Sauteria), et s'il continue à rester actif, le disque peut prendre une conformation toute particulière; dans les Marchantia, en effet, il se développe en rayons dont l'ensemble constitue un chapeau couvrant les archégones; ces derniers semblent alors insérés à la face inférieure du chapeau.

La formation des archégones peut arrêter (Blasia) ou ralentir seulement le développement de la branche qui les porte; il arrive aussi que cette branche redevient active après la fécondation et forme alors de no uveaux archégones (Fossombronia). Les organes sexuels ont en général une tendance à s'enfoncer dans des cavités par suite du soulève- 
ment du tissu ambiant; cela n'a lieu quelquefois qu'après la fécondation pour les archégones (Blasia); ces cavités ne s'ouvrent souvent au dehors que par d'étroits orifices; il se forme ainsi une espèce d'invo. Jucre. Mais l'ensemble de l'involucre et de son contenu peut aussi proéminer au-dessus du tissu environnant (Sphaerocarpus). Cet involucre (périanthe) se réduit, chez les Marchantia à une faible proéminence et ne se développe qu'après la fécondation de façon à entourer l'archégone; chaque groupe d'archégones possède ici encore en plus une enveloppe commune (périchèze). Très fréquemment aussi, les cellules placées entre les organes sexuels se déreloppent en poils (paraphyses).

Dans les Anthocerotées, anthéridies et archégones se forment dans l'intérieur du tissu. Pour les preniières l'assise extérieure du thalle se soulève dans une région circulaire au-dessus du tissu sous-jacent, d'où résulte un large espace intercellulaire au fond duquel s'élèvent des papilles, premiers rudiments des anthéridies; à la maturité le toit de la cavité se déchire. Les archégones proviennent d'une rangée de cellules perpendiculaire à la surface; cette rangée est issue de la division transversale d'un segment supérieur de la cellule terminale de la branche; elle devient plus riche en protoplasma; sa cellule inférieure se renfle et devient la cellule centrale, les autres forment le canal de l'archégone.

Dans les Marchantia, la fécondation se trouve notablement facilitée par ce fait, qu'au moment de la maturité, le pédoncule de l'inflores cence femelle est tellement court que le chapeau touche presque le thalle; les archégones, placés au bord, ont leur col recourbé en hatut; les groupes d'archégones correspondent de plus aux gouttières du chapeau; si donc une goutte de la bouillie anthéridienne arrive alors au sommet de l'inflorescence, la fécondation se trouve assurée. Ce n'est que plus 1ard que le pédoncule de l'inflorescence s'allonge, remplissant ainsi, pour la dispersion des spores, le même rôle que le pédicelle de la capsule des Mousses, lequel morphologiquement est tout différent.

Parmi les Jungermanniées à thalle, les Metzgeria portent leurs orgànes sexueis sur des rameaux adventifs, formés par la nervure médiane et très creusés à leur sommet. Dans les Jungermanniées foliacées, ces 
mêmes organes se montrent à l'extrémité des branches principales ou de petits rameaux particuliers qui naissent alors souvent par voie endogène sur la face ventrale de la tige; les anthéridies sont ordinairement axillaires des feuilles, isolées ou réunies en groupes. L.es archégones sont aussi produits par groupes au sommet des mêınes branches qui portent plus bas les anthéridies ou au sommet de branches exclusivement femelles; dans l'un et l'autre cas la cellule terminale de la branche se transforme toujours en un archégone (excepté Haplomitrium) d'où le nom de $J$. acrogynes, donné par M. Leitgeb aux J. foliacées. Dans certaines Jungermanniées dites Géocalycées, dans les Calypogeia surtout, ce sommet se creuse tellement que les archégones se trouvent enfoncés dans une sorte de cruche qui constitue alors un pseudopérianthe. Quand cette invagination n'a pas lieu, il se développe ordinairement, autour du groupe des archégones, un véritable périanthe en forme de calice; dans l'un et l'autre cas les feuilles les plus voisines constituent un périchèze en dehors de cette première enveloppe.

Mousses. - Anthéridies et archẻgones sont réunis en grand nombre à l'extrémité de l'axe feuillé; ils sont entremêlés de paraphyses dont la cellule terminale se renfle souvent en sphère, et entourés de feuilles à conformation particulière; celles-ci sont plus larges et plus dures dans les fleur's mâles où elles forment le périgone; dans les fleurs emelles ou bisexuées elles constituent le périchèze; elles ressemblent alors davantage aux feuilles ordinaires et diminuent de grandeur de dedans en dehors.

Quand les fleurs sont unisexuées, les pieds sont ou monoïques ou diö́ques; dans ce dernier cas les mâles peuvent être plus petits, n'avoir qu'une durée éphémère (Funaria hygrometrica, Dicranum undulatum, Leucobryum glaucum), et végéter sur le protonéma feutré des individus femelles. Les Mousses dioïques sont même parfois réduites à un seul sexe; stériles par conséquent, elles ne possèdent que la propagation végétative. Les fleurs mâles, souvent colorées en jaune ou en rouge, ont un aspect différent de celui des fleurs femelles et peuvent présenter la forme d'un bourgeon, d'une tête sphérique ou d'un disque 
(Schimper). Dans les Sphagnum, les anthéridies sont placées à côté des feuilles le long des branches màles qui prennent une conformation particulière etqui peuvent ensuite s'allonger de nouveau en branches ordinaires; il y a donc analogie avec les Jungermanniées; dans les Polytrichum, on a aussi observé un semblable accroissement, consécutif à la formation des anthéridies.

Dans les fleurs hermaphrodites archégones et anthéridies sont rapprochés au centre du périchèze, ou disposés en deux groupes distincts, ou séparés par des feuilles particulières qui portent les anthéridies à leur aisselle et sont ordonnées en spirale autour du groupe central des archégones.

Les anthéridies des Sphagnum naissent chacune à la place d'un rameau végétatif, à côté d'une feuille. Dans la plupart des fleurs mâles, la première anthéridie semble provenir de la cellule terminale, les suivantes de ses derniers segments, les autres de cellules superficielles. Le premier archégone provient de la cellule terminale et les suivants de ses segments.

\section{Développement du sporogone.}

La fécondation n'exerce pas seulement son influence sur l'œuf, mais aussi sur l'archégone et même au delà. Le ventre s'accroît avec l'embryon qu'il enveloppe dans les Hépatiques jusqu'à ceque son développement soit achevé. L'archégone ainsi modifié a été désigné sous le nom de coiffe; au moment de la maturation elle se trouve déchirée par la capsule, parsuite de l'allongement du pédicelle. Dans les Sphagnum le sporogone atteint aussi son développement presque complet dans le ventre de l'archégone accru; mais dans les autres Mousses, longtemps avant l'achèvement de la capsule, la coiffe est arrachée à sa base et soulevée au sommet du sporogone qu'elle couronne encore longtemps comme un bonnet rouge brun.

La première cloison de l'œuf est en général perpendiculaire à l'axe de l'archégnne; mais le développement ultérieur diffère assez notablement, ce qui oblige à l'examiner dans chaque division séparément.

Hépatiques. - Le cas le plus simple est celui des Riccia; l'œuf com- 
mence par se diviser en huit octants par trois cloisons perpendiculaires entre elles; la segmentation continue ensuite dans ces huit premières cellules, de façon à former un corps cellul aire, dont la couche périphérique reste stérile et constitue la paroi, bientôt résorbée, du sporogone, tandis que tout le tissu à l'intérieur de cette paroi se transforme en cellules mères des spores. Chaque cellulè mère se ảivise ensuite en quatre spores; les spores restent enfermées dans le ventre de l'archégone et ne devienrent libres que par la destruction du thalle. Le fruit offre une analogie évidente avec celui des Coléochétées (M. Pringsheim); son organisation est des plus simples; il n'y a encore aucune différenciation en pédicelle et capsule, et presque tout l'œuf se transforme en tissu fertile ou archéspore, laquelle se change entièrement en cellules mères.

Dans les autres Hépatiques le sporogone offre une complexité plus grande; on y distingue un pédicelle et une capsule, et de plus toutes les cellules de l'archéspore ne sont plus fertiles, mais employées soit à la nutrition des spores, soit à leur dispersion; dans ce dernier cas elles se transforment en élatères. Déjà dans la famille des Ricciées on trouve des différenciations dans ces deux sens; les Corsinia et Boschia montrent en effet un pédicelle et des élatères, réduits pour le premier genre à de petites cellules fusiformes, mais présentant déjà dans le second les propriétés caractéristiques; ils sont en effet allongès, bruns, munis d'épaississements annulaires ou spiralés, hygroscopiques. Dans les Sphaerocarpus les cellules stériles de l'archéspore sont moins différenciées, leur parois restent minces et elles semblent servir à la nutrition des cellules mères; l'embryon se divise en plusieurs disques superposés, ce qui correspond à la forme allongée du sporogone; sa partie supérieure donne la capsule, l'inférieure le pédicelle.

Dans les Marchantiées, la première cloison établit déjà la distinction entre le pied et la capsule; elle est ordinairement perpendiculaire à l'axe de l'archégone, quelquefois cependant aussi oblique. Puis viennent deux autres cloisons à peu près perpendiculaires à la première et entre elles; l'embryon se trouve alors divisé en huit octants; les quatre inférieurs forment le court pédicelle; les 
quatre supérieurs, tournés vers le col, se divisent par autant de périclines en cellules externes (calottes) qui sont l'origine de la paroi, et cellules internes qui forment les spores et les élatères; ceux-ci rayonnent ici de la base à la périphérie. La capsule se fend au sommet en nombreuses dents ou en quatre valves, ou s'ouvre par un opercule.

La première closson des Jungermanniées, toujours perpendiculajre à l'axe de l'archégone, ne sépare plus le pédicelle de la capsule; la cellule inférieure reste indivise ou subit seulement quelques segmentations; elle reste comme appendice à la base ơu pédicelle qui résulte, ainsi que la capsule, de la moitié supérieure de l'œuf. Celle-ci se montre bientôt formée par un certain nombre de disques transversaux, constitués en partie par division intercalaire, et composés chacun de quatre quartiers cylindriques; dans le disque supérieur ces quartiers sont des octants de sphère; mais ces quartiers ne se correspondent pas exacternent d'un disque à l'autre. Dans les cas les plus simples, c'est l'étage supérieur seul qui donne la capsule (Pellia, Frullania, Lejeunia), en coupant ces quatre cellules par autant de péricliries, ce qui fournit une couche extérieure, la paroi, et quatre octants sphériques intérieurs, l'archéspore; mais ordinairement les étages les plus rapprochés du preniier contribuent aussi à la formation de la capsule (Radula). Dans l'archéspore prennent naissance les élatères et les cellules mères. La partie inférieure de l'embryon continue ses divisions transversales pour constituer le pédicelle dont la base renflée pénètre assez profondément dans le tissu du rameau fructifère; le pied ainsi se trouve étroitement enveloppé par la vaginule. L'archéspore prend une forme arrondie, pendant la maturation des spores, la couche interne de sa paroi se trouve résorbée. Le pédicelle s'allonge notablement par extension de ses cellules (et sans divisions intercalaires); il déchire la coiffe et soulève en l'air la capsule qui s'ouvre en quatre valves longitudinales.

Le sporogone des Anthoceros se distingue par certaines particularités de celui des autres Hépatiques; sa durée est beaucoup plus longue, et pendant que son sommet, ouvert en deux valves, laisse déjà échapper les spores mûres, sa base continue, par accroissement intercalaire, à se développer et à former de nouvelles spores. Sa région 
inférieure stérile ne donne pas un pédicelle, mais coustitue un large pied dontles cellules extérieures, allongées en filaments, pénètrent profondément dans le tissu du thalle: celui-ci constitue un involucre autour du jeune sporogone qui le perce plus tard. La paroi de la capsule est riche en chlorophylle et possède des stomates qui manquent dans les autres Hépatiques, mais se trouvent fréquemment dans le sporogone des Mousses; comme la plupart de celles-ci, l'Anthoceros forme dans sa capsule une colımelle centrale. Tout en occupant ainsi une place particulière, l'Anthoceros se rattache néanmoins aux Jungermanniées par le genre Notothylas qui possède aussi une columelle.

L'œuf met vingt-quatre heures à s'entourer d'une membrane ; après huit jours seulement il commence à se segmenter; il se montre formé ensuite de deux, quelquefois trois étages, chacun de quatre cellules; l'étage inférieur constitue le pied; l'étage supérieur (ou les deux supérieurs) la capsule. Pour cela, les cellules de la capsule se divisent d'abord chacunє par une péricline en une externe et une interne; mais contrairement à ce que nous avons vu dans les autres Hépatiques, ce sont les cellules externes qui donnent ici naissance à l'archéspore, tandis que les internes se transforment en columelle. Les premières, en effet, se segmentent par de nouvelles périclines, d'où résultent deux calottes emboîtées l'une dans l'autre et recouvrant toutes deux la columelle; la calotte interne est l'archéspore qui a la forme d'une cloche comme dans les Sphagnum et les Andraea. Une faible partie seulement des cellules de l'archéspore est fertile; les autres constituent un réseau enveloppant les cellules mères (espèces indigènes), ou des élatères consistant en filaments transversaux pluricellulaires (espèces exotiques).

M. Leitgeb distingue donc quatre types de sporogenes dans les Hépatiques:

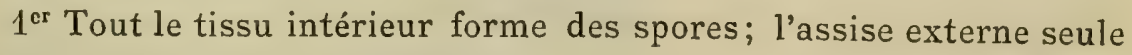
reste stérile (Riccia, Oxymitra);

$2^{e}$ Le tissu intérieur se différencie en cellules mères et en cellules nutritives (Corsinia, Rielia, Notothylas);

$3^{\mathrm{e}}$ Les cellules intérieures stériles se transforment en élatères (la plupart des Hépatiques); 
$4^{\mathrm{e}}$ Le tissu intérieur forme une columelle que la couche fertile recouvre comme une cloche (Anthoceros, Notothylas).

Mousses. - Dans quelques cas le sporogone ne diffère pas très notablement de celui des Hépatiques; en général il prend une structure bien plus compliquée. Le long pédicelle (soie) s'enfonce profondément par sa base dans le tissu de la tige fructifère. Le sporange (capsule, urne) qui le termine supérieurement possède une paroi à plusieurs assises et à épiderme différencié, une columelle axile coiffée quelquefois par l'archéspore qu'elle traverse le plus souvent et une archéspore ordinairement en forme de cylindre creux ou rarement en forme de cloche; de plus on distingue encore fréquemment un ou deux sacs sporifères (externe et interne) et des dispositions plus ou moins compliquées assurant la déhiscence ou préservant les spores contre l'humidité dans le sporogone ouvert : opercule, anneau, péristome.

C'est l'embryon des Sphagnum qui se rapproche le plus de celui des Hépatiques, il se divise d'abord par une cloison perpendiculaire à l'axe de l'archégone en une cellule inférieure qui se segmente peu et forme un appendice à la base du pédicelle, et une cellule supérieure qui constitue seule le sporogone; pour cela elle est coupée successivement par 6 à 8 cloisons transversales, plus ou moins obliques; les cellules résultantes se subdivisent par cloisons longitudinales; l'accroissement devient ensuite intercalaire.

L'embryon des autres Mousses se découpe aussi d'abord par une ou deux cloisons transversales 1-1 fig. 1; puis la celulle supérieure

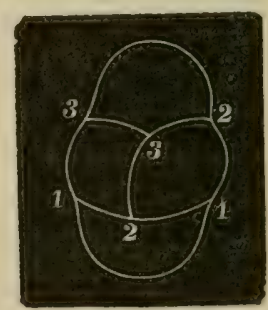

FIG. 1. - Coupe longitudinale de l'embryon d'une mousse.

se segmente cornme une cellule terminale à deux faces; pour cela une première cloison obliquernent transversale et courbe (anticline) 2-2 
s'appuie d'un côté sur sa face extérieure, de l'autre sur la cloison qui lui sert de base; une deuxième anticline $3-3$ se forme en sens opposé s'insérant en dedans sur la première et en dehors sur la paroi externe opposée à celle que découpait la première anticline, et ainsi de suite. Quand la cellule terminale a formé ainsi plusieurs segments, elle subit souvent une transformation analogue à celle de la cellule terminale du prothalle dans les Fougères. L'embryon se compose alors de plusieurs étages plus ou moins réguliers; une section transversale rencontre deux cellules; chacune se divise par une cloison radiale (2-2 fig. 2) et il y a ainsi quatre quartiers juxtaposés. Dans chaque

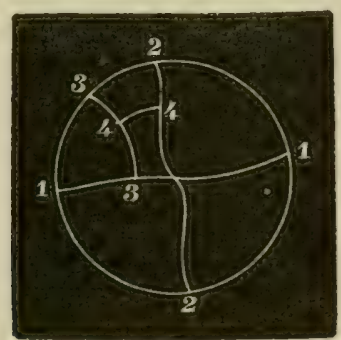

Fig. 2. - Coupe transversale de l'embryon d'une mousse.

quartier apparaissent alors successivement deux cloisons longitudinales : une anticline (3-3), puis une péricline (4-4); l'étage ou l'article se compose alors de quatre cellules internes et de huit externes. Un résultat analogue se trouve atteint d'une façon plus simple dans quelques mousses (Funaria, Ephemerum); l'anticline (3-3) ne se forme pas, la péricline (4-4) se prolonge jusqu'à la première cloison et l'étage comporte alors quatre cellules internes et quatre externes. Le premier ou les deux premiers étages situés au-dessus de la première cloison transversale de l'œuf sont destinés à former le pédicelle et ne présentent pas toujours une semblable segmentation; mais celle-ci apparaît régulièrement depuis le troisième étage, à partir duquel l'embryon se transforme en capsule. L'ébauche de l'urne est donc formée alors d'un cylindre plein entouré d'un cylindre creux; le premier, par l'apparition de périclines dans ses différentes cellules, donne naissance à un second cylindre creux emboîté dans le premier, c'est l'archéspore, et à une colonne massive centrale, c'est.la colu- 
melle. Le premier cylindre creux ou manchon externe se subdivise d'abord en plusieurs assises; puis apparaît dans son intérieur un espace intercellulaire (lacune aérifère) qui sépare deux assises internes (sac sporifère externe) de plusieurs assises externes qui constituent la paroi de la capsule. Quant au sac sporifère interne, il est fourni par la couche externe de la colonne massive, c'est-à-dire de la columelle.

L'archéspore, comprise entre les deux sacs sporifères, est traversée en haut par la columelle et présente donc, dans les Bryacées et les Phascacées, la forme d'un tonneau sans fond, ni cuuvercle ( $4^{\mathrm{C}} \mathrm{t} y \mathrm{pe}$, M. Leitgeb). Dans les Sphagnum et les Andraea elle a la forme d'une cloche couvrant la columelle; chez ces derniers cumme dans les familles précédentes, l'archéspore provient du premier cylindre plein ( $3^{\circ}$ type). Chez les Sphagnum au contraire elle est formée par la première assise périphérique de l'embryon (premier cylindre creux), ce qui rapproche les Sphaignes (1 ${ }^{\text {er }}$ type) des Anthoceros. Dans les Archidium, enfin, c'est encore le cylindre central qui donne naissance à l'archéspore; des cellules peu nombreuses ( 1 à 7 ) et quelconques de ce cylindre deviennent des cellules mères; tlles finissent par résorber toutes les autres cellules à l'intérieur de la paroi externe, réduite finalement elle-même à une assise unique; la columelle disparaît donc aussi ( $2^{\theta}$ type).

On a trouvé quelquefois deux sporogones portés sur un pédicelle commun (Meesea uliginosa par exemple). M. Leitgeb a montré qu'ils provenaient d'une oospore unique, par bifurcation de la cellule terminale de l'embryon; suivant que cette bifurcation se fait plus ou moins tôt, la séparation des deux capsules et de leurs soies est plus ou moins complète. D'après M. Leitgeb, ce serait le retıur à un état ancestral.

\section{GERMINATION DES SPORES.}

Les spores, en germant, ne donnent pas naissance directement à la plante sexuée, mais produisent d'abord un protonéma, beaucoup moins développé dans les Hépatiques que dans les Mousses ou il a 
souvent été pris autrefois pour une algue. La génération sexuée naît ordinairement comme une branche latérale sur le protonéma.

Dans le sporogone chaque cellule mère de spores se divise en quatre spores; cette partition se retrouve dans les spores des Cryptogames vasculaires et même dans les microspores des Phanérogames; dans les Anthoceros elle présente ce caractère particulier que le noyau ne se divise qu'après le protoplasma (M. Strasburger).

La spore est munie d'une double enveloppe : l'endospore, présentant la réaction de la cellulose, et l'exospore cuticularisée, de couleur foncée; celle-ci est verruqueuse dans le Grimaldia dichotoma où elle présente même une expansion vésiculeuse remplie d'air, rappelant les vésicúles des microspores dans les Abiétinées.

Dans les Mousses les spores contiennent de la chlorophylle et conservent longtemps, néanmoins, leur faculté germinative; elles en contiennent quelquefois aussi dans les Hépatiques (Fegatella). Les premières segmentations de l'embryon peuvent, dans quelques cas, avoir lieu dans l'intérieur de la spore (Pellia, Fegatella, Andraea).

A la germination, l'enveloppe de la spore est rompue, et son contenu s'allonge en un filament entouré d'une mince membrane; c'est le protonéma. Il est rudimentaire dans les Hépatiques; très développé au contraire dans les Mousses.

Hépatiques. - La plante sexuée apparaît ordinairement comme un bourgeon latéral sur le filament cloisonné, quelquefois aussi elle en constitue le prolongement direct. Dans les Ancura, le protonéma se segmente d'abord transversalement, puis dans sa cellule terminale apparaissent deux cloisons obliques, découpant la cellule terminale à deux faces du thalle sexué. Dans les Pellia oủ les premières divisions ont lieu à l'intérieur de la spore, il se forme d'abord un corps cellulaire dont une cellule s'allonge en rhizoïde; une autre, opposée à la première, devient la première ébauche de la plante. Dans les Jungermanniées foliacées, le protonéma peut être un filament cellulaire unisérié (Lophocolea, Chiloscyphus) quelquefois ramifié, dont la cellule terminale devient la cellule initiale à trois faces de la tige feuillée; ou bien il consiste en une lame cellulaire (Radula, Frullania), dont une cellule marginale se développe en plante sexuée. 
La spore des Marchantiées n'émet qu'à la lumière un filament germinatif pluricellulaire, héliotrope, d'autant plus court que l'éclairage est plus intense; son extrémité se renfle et s'élargit perpendiculairement aux rayons lumineux; elle se transforme pour cela en un disque résultant de la division en octants de la cellule terminale. Des cellules marginales de ce disque deviennent les initiales du thalle; au commencement, sa structure est fort simple et il possède tout d'abord une cellule terminale à deux faces.

Mousses. - I.e protonéma, très développé, peut devenir la partie prépondérante de la plante. Il se compose de filaments confervoïdes, unisériés, rameux, sur lesquels la génération sexıée prend naissance comme un rameau lateral de structure beaucoup plus compliquée; à l'inverse des Hépatiques, plusieurs tiges peuvent se former sur le même protonéma.

Ordinairement il émerge d'abord de la spore un seul filament à accroissement terminal indéfini, sans accroissement intercalaire; toutes ses cellules peuvent émettre des branches qui s'allongent aussi indéfiniment en se ramifiant à leur tour. Ce n'est que postérieurement à ce premier filament qu'il en naît un deuxième à l'extrémité opposée de la spore (quelquefois même plusieurs); celui-là devient souvent un rhizoïde; du reste, toutes les parties du protonéma peuvent subir pareille transformation en s'enfonçant dans le sol.

La genération sexuée provient de la cellule basilaire d'une branche latérale à accroissement défini, rarement de sa cellule terminale; pour cela un refoulement latéral de cette cellule s'en sépare par une cloison et, se segmentant rapidement, devient un bourgeon foliaire.

Quelquefois le protcnéma a une existence presque indéfinie; dans certaines Phascacées, il subsiste encore après la production des tiges feuillées, et jusqu'à l'achèvement des spores. Mais ordinairement il disparaît après la formation de ces tiges.

Quelquefois le protonéma affecte une forme différente; dans les Diphyscium, Tétraphis, ses filaments développent latéralement des lames foliacées. D'autres fois, l'axe même du protonéma se change en une lame cellulaire étalíe, dont la surface donne naissance aux tiges 
feuillées (Sphagnum), ou bien constitue d'abord un corps cellulaire (Andraea), dent une à trois cellules périphériques émettent autant de filaments; ces filaments se changent en lames cellulaires ramifiées, pouvant émettre de nouveaux filaments.

\section{PROPAGATION VÉGÉTATIVE.}

La propagation végétative acquiert dans le groupe des Muscinées, et surtout dans la classe des Mousses, une importance qu'elle ne possède dans aucune autre division du règne végétal (M. Sachs). Ses modes sont très variés.

Le thalle ou la tige feuillée s'accroît incessamment par la pointe, les parties âqées meurent, et il en résulte que les diverses branches se trouvent séparées en autant de plantes autonomes. Cette multiplication devient encore plus importante par la formation de branches adventives; elles naissent aux dépens des cellules marginales du thalle, plus rarement des cellules superficielles de la nervure médiane (Metzgeria, Sphaerocarpus); quelquefois aussi leur formation est endogène ou plus exactement hypodermique (Metzgeria). Des pousses adventives proviennent aussi de la face ventrale de İa tige dans les Hépatiques foliacées; quand elles doivent leur origine à une cellule superficielle, celle-ci s'allonge en un long filament, à l'extrémité duque apparait un bourgeon. Dans les Marchantiées, de pareilles formations peuvent se montrer jusque sur les inflorescences, notamment sur les gouttières à rhizoïdes des inflorescences mâles. Il y a même des branches (Conomitrium julianum, Cinclidotus aquaticus) et des bourgeons normaux (Bryum annotinum) qui se détachent spontanément de la tige, et reproduisent de nouvelles plantes.

Les propagules constituent un très important mode de multiplication; ils germent comme les spores, et commencent toujours, dans les Mousses, par former un protonéma ramifié. Ils peuvent se réduire à une cellule marginale ou superficielle (face dorsaic) qui se détache de ses voisines, s'arrondit et se segmente en deux pour ne continuer son développement que quand elle arrive sur le $3 \mathrm{c}_{2}^{i}$ (Aneura); dans 
les Madotheca de pareilles cellules sont très nombreuses au bord du thalle. D'autres fois les propagules ne prennent naissance que dans des conceptacles particuliers à la face supérieure du thalle; au fond de ces conceptacles s'élèvent des papilles dont la cellule terminale se dévelopse en un massif cellulaire porté par un pédicelle; ces papilles sont entremêlées de poils sécrétant un mucilage, lequel, en se gonflant, repousse peu à peu hors du conceptacle les propagules détachés de leur pédicelle. La lumière est indispensable pour la germination de ces organes (Blasia, Lunularia, Marchantia).

Les propagules uni ou paucicellulaires des Jungermanniées foliacées se forment sur les feuilles et les tiges, mais surtout. à l'extrémité des feuilles; souvent celles-ci se trouvent complètement envahies ou même entièrement remplacées par un groupe de ces corps reproducteurs (Jungermannia, Scapania, Calypogeia). Les bords des feuilles de Radula complanata donnent naissance à des propagules pluricellulaires, qui émettent des tiges feuillées de la nıême manière que les lames issues des spores (voir plus haut). Enfin les feuilles peuvent produire directement des plantes nouvelles (Lophocolea).

Dans les Mousses on trouve des propagules pédicellés, pluricellulaires, fusiformes ou lenticulaires ; ils naissent au sommet d'un prolongement aphylle de la tige feuillée (Aulacomnium androgynum); dans le Tetraphis pellucida, ils sont enveloppés d'un calice formé de plusieurs feuilles délicates.

Ils se développent, entremêlés de poils (M. Berggren), aux dépens chacun d'une cellule qui se divise en deux, et dont la moitié inférieure forme le pédicelle, tandis que la moitié supérieure devient une cellule terminale à deux faces, qui produit tout le progagule. A la germination, ces corps émettent des filament de protoriéma, sur lesquels apparaissent les tiges feuillées; il provient donc plusieurs tiges de chaque propagule. Dans le Bryum annotinum ces corps ovales, rouges, longuement pédicellés, naissent à l'aisselle des feuilles, dans les Grimmia à leur pointe, dans les Barbula sur leur nervure.

Mais dans les Mousses, le protonéma lui-même est l'organe le pius actif de multiplication; sur ses diverses branches, il produit sinultanement et progressivement de nombreuses tiges feuillées ; sou- 
vent aussi des propagules ou bourgeons tuberculeux (Bryum, Fissidens) ; desséché, il se divise en fragments dont quelques-uns s'entourent d'une épaisse membrane, et germent par le retour de l'humidité. Tout poil radical peut produire un protonéma secondaire ou même directement des bourgeons foliaires, quand il est placé à la lumière dans une atmosphère humide, et certaines espèces, en apparence annuelles, sont en réalité vivaces par ce moyen (Phascum, Funaria, Pottia).

Le protonéma provient souvent directement des feuilles (Orthotrichum, Grimmia, etc.), ou de la tige (Oncophorus glaucus).

La face interne de la coiffe donne même naissance à de courts filaments protonématiques, sur lesquels se forment très vite des plantes feullées, qui sortent de dessous la coiffe, et qui ne tardent pas à fructifier à leur tour (Schimper : Synopsis); il parait que de pareilles coiffes peuvent couvrir des sporogones normalement développés.

MM. Pringsheim et Stahl ont, d'un autre côté, obtenu des protonéma par la culture de sporogones, découpés en morceaux, ce qui revient a supprimer la spore intermédiaire entre le sporogone et le protonéma, et à greffer celui-c1 sur la génération asexuée, résultant de la fécondation (Hypnum, Bryum, Ceratodon).

La propagation végétative acquiert donc dans les Muscinées, et surtout dans les Mousses, une très grande importance; ce qui le démontre encore, c'est que, dans certaines Mousses, on ne trouve que rarement (ou même jamais) des spores et des sporogones, soit parce qu'il ne se forme ni archégones, ni anthéridies (Barbula papillosa); soit parce que l'un des sexes manque totalement, au moins dans certaines contrées (Lunularia vulgaris), ou est devenu très rare (Ulota phyllantha) ; soit parce que les deux sexes ne se trouvent que rarement réunis (Leucobryum glaucum).

La fécondation chez les Muscinées n'est possible que par l'intermédiaire de l'eau. Les Mousses étant des plantes terrestres, leur station est tout à fait défavorable à l'accomplissement de l'acte sexué; leurs dimensions réduites, l'extrême petitesse de leurs organes sexuels rémédient en partie à ces inconvénients, en rendant la fécondation pos-. 


$$
-134-
$$

sible par l'irtermédiaire de la pluie, de la rosẻe, des insectes, etc. L'abondante propagation végétative agit dans le même sens; en réunissant ensemble un nombre énorme d'individus, elle favorise évidemment la fécondation, et c'est là, sans doute, ce qui explique comment, malgré ce mode de fécondation, les Mousses ont pu s'adapter à la vie terrestre. 


\section{CRYPTOGAMES VASGULAIRES}

Le développement complet des Cryptogames vasculaires comprend, comme dans les Muscinées, deux générations, l'une sexuée, l'autre asexuée; les organes de la reproduction offrent également dans les deux groupes une très grande analogie; malgré cela, il existe entre eux des différences telles qu'il faut les considérer comme deux branches divergentes, soient soudées primitivement à la base, soit émergées toutes deux séparément des alģues. En effet, nous avons vu que dans les Muscinées la génération asexuée, le sporogone, reste constamment fixé sur la génération sexuée, dont elle est en quelque sorte le parasite; elles offrent l'une et l'autre une différenciation progressive depuis les Hépatiques les plus inférieures jusqu'aux Mousses les plus élevées, mais cette différenciation est plus accusée pour la génération sexuée (le prothalle). Celle-ci, en effet, présente finalement des tiges, des feuilles et presque des fleurs; on y distingue de plus des cellules de forme et de structure diverses; certains éléments même, allongés et réunis en cordons, peuvent être considérés comme les précurseurs des faisceaux fibro-vasculaires.

Dans les Cryptogames supérieures, au contraire, c'est la génération asexuée, c'est le sporogone, qui nous offre de beaucoup la plus grande différenciation tant anatomique qu'histologique; il est bien encore parasite, dans son premier âge, du prothalle; mais il ne tarde pas à s'en affranchir, à mener une vie indépendante et à acquérir dès lors un développement croissant. Tout au contraire le prothalle, toujours de structure très simple, se réduit progressivement, et si, dans les régions inférieures de notre groupe, il constitue encore une plantule à nutrition indépendante, nous le verrons, dans les régions supérieures, devenir unisexué et n'être plus qu'une simple émanation, un pa- 
rasite de la spore. Celle-ci cependant se détache encore du sporogone, de sorte que les deux générations sexuée et asexueje sont toujours ici deux individualités distinctes. Il suffit cependant de faire un pas de plus, il suffit que la spore femelle reste fixée avec son sporange (nuceile) sur le sporogone, que le prothalle, encore plus réduit, demeure inclus dans sa spore, pour que les deux générations se trouvent de nouveau confondues en un tout continu, comme dans les Mousses, mais en sens inverse; c'est la génération sexuée qui sera greffée ici sur la plante asexuée que l'œuf même n'abandonnera qu'après avoir ébauché déjà toutes les parties essentielles d'une plante nouvelle, qu'après s'être transformé en petite plantule embryonnaire; de sorte que le sporogone semble succéder au sporogone avec suppression apparente de la génération sexuée. Ce sont là les phénomènes que présentent les Phanérogames où les spores mâles (grains de pollen) se detachent seuls de leur sporange.

Les Cryptogames vasculaires nous conduisent donc naturellement par leurs types les plus élevés aux Gymnospermes et aux Angiospermes; par contre leurs formes inférieures ne se rattachent que de loin ' $1 \mathrm{x}$ formes inférieures des Hépatiques ou même aux Algues, et entre les deux grands groupes des Muscinées et des plantes vasculaires, il sxiste une véritable divergence; mais cette divergence il faut la chercher dans les considérations précédentes; car dans les appareils spéciaux de la reproduction, spores, archégones et anthéridıes, il existe la plus grande analogie, surtout à la base de la série que nous allons parcourir.

On peut diviser les Cryptogames vasculaires en trois grandes classes: Equisétinées, Filicinées et Lycopodinées, dont chacune se compose, inférieurement, de familles n'ayant qu'une seule espèce de spores ou Isosporées, et supérieurement, de familles ayant deux espèces de spores, mâles et femelles, ce sont les Hétérosporées.

Dans les Isosporées, nous trouvons: les Equisétacées actuelles, les Fougères, les Lycopodiacées.

Dans les Hétérosporées, certaines Equisétinées fossiles, les Rhizocarpées, Sélaginellées et lsoétées.

Nous allons entreprendre l'examen comparatif des divers organes 
reproducteurs dans ces classes, en laissant de côté seulement les Equisétinées hétérosporées, qui sont en dehors de notre sujet.

\section{SPORANGES.}

La plante asexuée des Cryptogames vasculaires, composée d'une tige, de feuilles el de racines, équivaut morphologiquement au sporogone des Mousses, mais physiologiquement ce dernier se trouve représenté par les sporanges.

Tantôt les sporanges sont des productions de la face inférieure des feuilles (la plupart des Fougères) ou de leurs bords (Hyménophyllacées); tantôt ils sont placés sur l'axe portant les feuilles et alors à la base interne d'une feuille (Lycopodium) ou un peu au-dessus de cette base (Selaginella); ils peuvent même dans le seconả cas n'avoir aucun rapport avec les feuilles (Psilotum). Les sporanges ne doivent donc pas être considérés, dans tous les cas, comme des émergences de feuille, pas plus dans les Cryptogames vasculaires que dans les Phanérogames.

Les sporanges peuvent être sessiles ou pédicellés; mais toujours on distingue une enveloppe et un tissu sporigène ou archéspore (M. Göbel), séparés l'un de l'autre par une formation passagère, le tapis, qui correspond à l'endothèque des sacs polliniques. Leur déveJoppement a été le sujet de nombreux travaux dans ces dernières années (voir l'Index).

Dès les premiers états du développement a lieu la différenciation en enveloppe et en archéspore, cette dernière caractérisée par sa richesse en protoplasma, peut toujours être ramenée soit à une cellule hypodermique unique, soit à une rangée ou à une couche de cellules nypodermiques; toujours aussi par des bipartitions répétées, elle donne naissance à un tissu sporigène composé de cellules mères des spores dont chacune formera quatre spores.

Le tapis ou endothèque provient soit de l'archéspore elle-même, soit des cellules qui l'environnent, enfin il peut avoir cette double origine; il se compose en général d'éléments tabulaires dont les pa- 
jois sont résorbées pendant la formation des cellules mères des spores ou peu après cette formaticn; les rnasses protoplasmiques de ces cellules se fusionnent pour être employées ultérieurement à l'achèvement des spores.

La formation des spores par leur's cellules mères présente la plus grande analogie avec celle des microspores ou grains de pollen des Phanérogames; elle a été très soigneusement étudiée par M. Strasburger sur le Psilotum. Le noyau se divise en deux, la lame granuleuse apparaît, mais pour disparaître bientôt et la cloison définitive ne prend pas naissance. Les deux nouveaux noyaux se divisent à leur tour, généralement dans le même plan que le premier; les quatre noyaux de seconde génération s'écartent de façon à toucher presque la paroi de la cellule mère, les lames granuleuses correspondantes apparaissent et c'est à ce moment seulement que les trois cloisons des cellules définitives se forment [simultanément et coupent le protoplasma en quatre masses munies chacune de son noyau; il s'agit donc presque d'une quadripartition simultanée. Les matériaux de ces cloisons définitives sont sans doute fournis, en partie au moins, par les meinbranes résorbées des cellules du tapis. Plus tard, les spores s'entourent chacune de sa paroi particulière, et les cloisons sont résorbées ainsi que les parois de la cellule mère, de sorte que les spores deviennent libres dans l'intérieur de la cavité du sporange.

FILICINÉES ISOSPORÉES OU FOUGÈRES.

I.es sporanges sont toujours portés par des feuilles ou frondes spéciales; ces feuilles fertiles peuvent être semblables aux stériles on dissemblables; c'est ainsi que, dans le Struthiopteris germanica, elles sont petites et placées au milieu d'un entonnoir formé par les feuilles stériles bien plus grandes, que dans le Biechnum Spicant elles sont plus longues et dressées, que dans beaucoup d'Osmunda, Polybotrya, elles se réduisent presque aux nervures, etc. C'est surtout par la disposition des nervures que les deux espèces de feuilles diffèrent ordinairement.

Très rarement les sporanges se développent sur les deux faces de la 
feuille (Polybotrya cervina); c'est aux bords ou à la face inférieure qu'on les trouve généralement. Ils sont aussi presque toujours localisés le long des nervures; les Acrostichées font seules exception. Ils sont isolés ou réunis en sores; ceux.ci à leur tour pourront être nus ou munis d'une indusie latérale supère, infère, vraie ou fausse. Ces différents caractères sont utilisés par la botanique systérnatique, ainsi que les particularités de l'anneau.

Dans les Ceratopteris (M. Kny) les sporanges sont isolés sur la face inférieure de la feuille ; mûrs ce sont des capsules pédicellées, sphériques, qui s'ouvrent par une large fente transversale. Ils prennent naissance en direction acropète sur les feuilles encore enroulées. La première ébauche consiste en une cellule superficielle formant une papille proéminente et se divisant en deux par une cloison transversale plus ou moins oblique; la cellule supérieure plus grande est coupée alurs par trois cloisons à peu près perpendiculaires à la première, mais convergentes en bas et disposées en triangle, puis par une quatrième cloison à peu près parallèle à la première transversale. La papi'le se compose alors de trois cellules périphériques, d'une cellule calotte et d'une cellule centrale à peu près tétraédrique qui est l'archéspore; le tout est porté par la cellule basilaire qui, s'allongeant et se segmentant, donne le pédicelle, composé de deux ou trois rangées de cellules. Par quatre cloisons à peu près parallèles à ses quatre faces, l'archéspore donne naissance à quatre cellules tabulaires composant le tapis et se subdivisant ultérieurement en plusieurs assises. Par bipartitions répétées la cellule centrale donne les cellules mères des spores au nombre de quatre ou d'un multiple de quatre; puis ces cellules mères forment chacune quatre spores, en même temps il y a résolution des cellules du tapis ou endothèque.Les cellules de l'enveloppe de la capsule, destinées à former l'anneau, se segmentent plus longtemps que leurs congénères par des cloisons rayonnantes, jusqu'à ce que le nombre définıtif des éléments de l'anneau se trouve atteint; ces éléments s'allongent alors radialement, épaississent celles de leurs parois transversales par lesquelles elles tcuchent aux autres cellules de l'anneau, ainsi que leur paroi interne; puis prennent une couleur 
jaunâtre. Par la dessiccation cet anneau se contracte et déchire la capsule, ce qui met les spores en liberté.

Ce développement peut servir de type pour les Hyménophyllacées, Cyathéacées et Polypodiacées; seulement les sporanges sont ici généralement réunis en sores. Dans les Hyménophyllacées, le sore occupe l'extrémité d'une nervure qui dépasse le limbe et qui forme un réceptacle ou columelle; il est entouré d'une indusie non fermée au sommet, composée de deux lèvres plus ou moins soudées qui correspondent aux deux faces de la feuille et qui reçoivent chacune un faisceau rudimentaire. Des glandes pédicellées sont implantées sur le réceptacle, plus rarement sur les pédicelles des sporanges. Pour la formation de l'indusie plusieurs cellules marginales se divisent chacune en deux par une cloison parallèle à la surface de la feuille, les cellules filies continuent à se segmenter par des cloisons radiales et des cloisons parallèles à la première ; il en résulte un amas cellulaire dont les bords s'accroissent plus vite formant les lèvres de l'indusie; la columelle apparaît dans l'excavation médiane.

Dans les Cibotiacées, l'indusie est à plusi®urs assises, ses deux lèvres sont dissemblables, et la columelle peut présenter un sporange terminal, ce qui est très rare dans la famille précédente.

Au lieu de terminer la nervure, le réceptacle peut en être une ramification latérale, perpendiculaire à la feuilie, tout en contenant encore des vaisseaux scalariformes, c'est ce que nous présentent les Cyathea, Alsophila, et certaines Polypodiacées (Aspidium filix mas); mais dans cette famille le réceptacle devient tout à fait rudimentaire.

Dans le Pleris aquilina Je réceptacle (M. Burck) est toujours la continuation du bord de la feuille, et il existe encore une véritable indusie; le prétendu bord recourbé de la feuille est en réalité la lèvre supérieure d'une vraie indusie dont la lèvre inférieure est réduite à une assise unique de cellules; si cette indusie possède ici un aspect si différent, c'est parce que les sores forment une rangée continue sur le bord de la feuille (Prantl). Dans le Gymnopteris aurita, c'est encore ce bord qui constitue le réceptacle, mais l'indusie a disparu. Dans les autres Pteris, il n'y a plus qu'une fausse indusie constituée par la 
feuille elle-mème repliée en dessous; les sores ne sont plus nullement individualisées, le réceptacle a disparu. Dans les Polypodiacées, les sores abandonnent ensuite le bord de la feuille et se disposent à l'extrémité des nervures, ou simplement sur le dos.

Parmi les Schizéacées le Lygodium présente encore des sporanges isolés, renfermés chacun dans une poche qu'on a comparée aux téguments de l'ovule des Phanérogames. G'est une cellule marginale qui donne naissance au sporange; les cloisons qui découpent la cellule centrale ne sont plus dirigées dans trois sens, comme pour le Ceratopteris, mais dans deux directions seulement, et cette particularité se retrouve (M. Prantl) dans tous les sporanges produits par des cellules de bordure qui possèdent done, comme la feuiìle elle-même, le caractère bilatéral. Plus tard seulement le sporange se trouve placé sur la face inférieure de la feuille. Le lobe supérieur de la poche est formé par le limbe même de la feuille, mais le lobe infárieur est une ėmergence spéciale. Les autres Schizéacées sont dépourvues d’indusie. Les sporanges se forment ici en direction acropète, tandis que, dans l'intérieur des sores à plusieurs sporanges, le développement de ceux-ci est basipète; aussi M. Prantl considère-t-il les sporanges isolés comme équivalents à des sores.

Dans les Osmundacées, les sporanges sont réunis en sores dépourvus d'indusie et placés à l'extrémité des nervures.

Dans les Gleicheniacées les sores, sans indusie aussi, occupent la face inférieure de la feuille fertile.

Dans les Marattiacées les sporanges s'écartent assez notablement de ceux des autres Fougères. L'Angiopteris forme la transition; dans ce genre, les sporanges ne sont plus pédicellés, mais sessiles sur un réceptacle très peu élevé (placenta, M. Göbel). Disposés sur deux rangs le long de la nervure fertile, ils s'ouvrent chacun par une fente longitudinale sur la face interne; mais ils ne se soudent pas dans un même sore. Dans les Marattia, au contraire, ces sporanges, toujours bisériés, se soudent intimement dans chaque sore. Celui-ci se divise à la maturité en deux moitiés, puis chaque sore s'ouvre encore sur la face interne par une face longitudinale.

Dans les Danaea le sore reste indivis, mais chaque sporange pos- 
sède une déhiscence porricide apicale. Dans le Kaulfussia acsculifolia les sporanges sont disposés en cercle avec une fossette centrale dans laquelle ils s'ouvrent. Il y a ici une indusie, de même que dans le genre précédent; dans les Marattia et Angiopteris cette indusie reste rudimentaire.

Dans les feuilles très jeunes, encore enroulées (Marattia) ou moins jeunes (Angiopteris), le coussinet ou placenta est déjà indiqué sur le dos de la nervure; là, en effet, un petit groupe de cellules forme une protubérance dans laquelle une cellule hypodermique constitue la première ébauche de l'archéspore; celle-ci fait partie d'une rangée de cellules (MM. Tschistiakoff, Göbel), perpendiculaire à la surface de la protubérance. Cette rangée ici n'est pas axile, mais latérale et asymétrique.

La cellule épidermique placée au-dessus de l'archéspore se segmente par des cloisons rectangulaires, et les éléments qui en résultent continuent ce mode de division, de sorte que l'archéspore se trouve plongée bientôt dans l'intérieur du tissu; elle ne se divise pas aussi régulièrement que dans les Polypodiacées, et ne donne pas naissance au tapis, qui est formé par le tissu environnant (MM. Luerssen, Göbel). L'enveloppe de la capsule se compose toujours de plusieurs assises de cellules.

Dans les Ophioglossées le développement se fait d'une façon analogue, la structure des sporanges est aussi semblable à celle des Marattiacées, seulement tout le segment foliairé fertile prend un aspect particulier.

Dans le genre Botrychium la première ébauche du sporange apparait sur le bord latéral du segment foliaire fertile (M. Göbel); par accroissement inégal des deux faces de ce segment il se trouve plus tard reporté surla face supérieure. Cette ébauche se compose, comme dans le cas précédent, d'un groupe de cellules formant une protubérance hémisphérique. Le point de départ de l'archéspore est encore une cellule hypodermique, remarquable par sa richesse en protoplasma finement granuleux; cette cellule augmente de volume et se divise en quatre par deux cloisons rectangulinaire, toutes deux lontigudinales par rapport à la feuille. La cellule épiderınique qui recou- 
vre l'archéspore, se divise par une cloison longitudinale en deux cellules juxtaposées dont chacune, par une série de transversales, forme une rangée de cellules perpenđ̊iculaire à la surface. Les deux assises inférieures de ces séries prennent part à la formation du tapis qui se constitue entièrement comme dans les Marattiacées, sans intervention de l'archéspore. Celle-ci est encore enfoncée à l'intérieur du sporange et recouverte d'une enveloppe à plusieurs assises. Toute la protubérance sporangienne s'arrondit et se soulève sur un très court pédicelle. La déhiscence s'opère seulement un an après par une fente transversale, au sommet; le développement de ces sporanges est acropète.

Il en est de même pour ceux des Ophioglossum; ils sont moins proéminents, dépourvus de pédicelle et confondus presque avec le tissu de la feuille. Leur paroi se compose de plusieurs assises dont la plus externe se continue avec l'épiderme de la feuille. La fente transversale de déhiscence est indiquée par des cellules plus petites. Dans les plus jeunes états observés jusqu'à présent l'archéspore ( $M$. Göbel) se composait déjà d'un groupe de cellules remarquables par leur grand noyau et par leur plasma dense, finement granuleux, riche en amidon; elles se distinguaient peu des callules épidermiques placées immédiatement au-dessus; le groupe entier provenait évidemment de trois cellules, pouvant probablement, comme dans le Botrychium, se ramener à une cellule unique que $\mathbf{M}$. Göbel suppose appartenir ici non à l'hypoderme, mais à l'épiderme. Plus tard, il se forme encore un tapis. Les sporanges mûrs sont recourbés transversalement et ressemblent beaucoup à certains sacs polliniques de Phanérogames.

FILICINÉES HÉTÉROSPORÉES OU RHIZOCARPÉES.

Dans cette sous-classe nous trouvons de petites spores mâles ou microspores et de grandes spores femelles ou macrospores; elles sont placées dans des sporanges différents et ceux-ci se trouvent réunis dans des conceptacles fermés.

Dans les Salvinia quatre à huit de ces conceptacles sont insérés en- 
semble sur une feuille submergée; ils sont arrondis, un peu aplatis transversalement, couverts extérieurement de poils et marqués de 9 à 14 côtes saillantes; à ces còtes correspondent autant de canaux aérifères séparés seulement entre eux par une couche de cellules. La paroi interne du conceptacle est munie de stomates; du fond de ce fruit s'élève le réceptacle ou columelle dont l'extrémité renflée supporte les sporanges. Un ou deux fruits du groupe ne contiennent que des macrosporanges au nombre de 10 à 20 dans chacun; tous les autres ne renferment que des microsporanges bien plus nombreux. Les mirrosporanges sont notablement plus petits, arrondis, longuement pédicellés; les macrosporanges ovales n'ont que de courts pédicelles. Dans les uns et les autres, l'enveloppe de la capsule est composée d'une seule assise de cellules tabulaires, polygonales et brunes à la maturité. Chaque conceptacle peut être considéré comme un segment de feuille métamorphosée, et est entièrement comparable à un sore d'Hyménophyllacée munie de son indusie.

Le développement (M. Juranyi) de chaque conceptacle commence de bonne heure sur les feuilles submergées encore très jeunes, non loin du cône végétatif; certains segments de feuille, à peine ébauchés, se renflent légèrement pour donner la future columelle, tandis que, à la base du segment, se forme, tout autour de cette columelle, un bourrelet annulaire par segmentation rapide des cellules épidermiques; c'est l'enveloppe du fruit ou l'indusie; d'abord en forme d'entonnoir, elle dépasse bientôt la columelle en se rétrécissant à sa partie supérieure qui ne tarde pas à se fermer. Les sporanges se forment aux dépens des cellules superficielles du réceptacle; ces cellules proviennent plus ou moins directement de la cellule terminale qui cesse alors de se segmenter. Elles se soulèvent en forme de papilles, et la succession des cloisons est très analogue, jusqu'd la formation des cellules mères des spores, à ce que nous avons vu chez les Fougères.

Dans les microsporanges le pédicelle est unisérié, dans les macrosporanges ses cellules se divisent par des cloisons longitudinales. Dans les premières, chacune des seize cellules mères donne quatre spores disposées en tétrades; les cellules de l'endothèque se résolvent en une gelée qui enveloppe ici l'ensemble des spores. Dans les macro- 
sporanges, chacune des seize cellules mères donne encore une tétrade; mais une seule des soixante-quatre cellules filles se développe ultérieurement; elle augmente de volume, s'arrondit, sor enveloppe devient jaune, puis brune. Son protoplasma qui n'a pas augmenté en proportion, ne forme bientôt plus qu'une mince couche à l'intérieur de la paroi ; vers la fin du développementil s'amasse en majeure partie à l'extrémité de la spore tournée vers le sommet du sporange où il enveloppe le noyau volumineux. Les cellules de l'endothèque, riches en contenu plasmique, s'accroissent d'abord notablement, formant une enveloppe continue autour du tissu sporigène; puis elles fusionnent entre elles et avec les spores non développées et la macrospore se trouve alors placée au milieu d'une masse plasmique qui durcit, se creuse de nombreuses petites vacuoles et prend ainsi un aspect spumeux; on dirait alors qu'elle est composée de petites cellules. C'est là l'enveloppe extérieure de la macrospore, c'est-à-dire l'épispore. Au sommet, elle se renfle en un cône dans lequel on distingue bientôt trois plaques verticales convergentes, distantes de $120^{\circ}$; elles marquent les lignes de déhiscence futures.

Le genre Azolla forme, a vec le précédent, la famille des Salviniacées; ici deux à quatre conceptacles sont insérés sur la feuille inférieure de la tige. Le sore femelle, dans son indusie, ne contient qu'un seul macrosporange, même dans les premiers états du développement; il est placé au sommet de la columelle. L'épispore est formée, non seulement par les cellules de l'endothèque et par les spores avortées, mais aussi par toute la partie inférieure résorbée de l'enveloppe sporangienne; de sorte que la spore unique se trouve placée directement dans l'indusie dont elle n'occupe que la moitié inférieure; cette épispore est encore dure, spumeuse, et constitue autour de la spore une enveloppe épaisse creusée d'excavations; au sommet de la spore elle se différencie en un appareil natatoire composé de trois masses piriformes, simples ou trilobeies, suivant les esprèces. Ce flotteur porte à sa partie supérieureles restes de l'enveloppe sporangienne; celle-ci à son tour est recouverte par une masse composée de filaments minces, feutrés, résistants, laquelle pénètre en bas entre les trois masses du flotteur et forme en haut, par-dessus tout l'appareil, une coiffe 
fibreuse. La coiffe descend en s'amincissant sur ies flancs du flotteur où elle finit par se fusionner avec l'indusie; cette soudure a lieu sur un anneau horizontal qui marque en même temps la limite entre la portion supérieure lignifiée de l'indusie et sa portion inférieure non lignifiée.

ILes fruits mâles renferment jusqu'à quarante microsporanges portés chacun sur un pédicelle biserié; sous l'enveloppe capsulaire, composée d'une seule couche de cellules, la masse plasmique spumeuse provenant de l'endothèque, s'est retractée en trois à huit ballès ou massulae arrondies, englobant chacune quatre à huit spores, et portant, à leur surface, des prolongements filiformes, pointus ou recourbés en crochets.

Dans les Marsiliacées, les fruits ou sporocarpes plus complexes présentent, sous une enveloppe commune, la réunion d'un plus ou moins grand nombre de sores.

Dans les Pilularia, la cavité du fruit est divisée en deux, trois ou quatre loges parallèles à l'axe; chaque loge porte à sa face périphérique un cordon (nervure) saillant à l'intérieur, sur lequel sont insérés de nombreux sporanges; les inférieurs sont des macrosporanges à une seule macrospore, les supérieurs des microsporanges à nombreuses microspores. Chacune de ces loges peut donc être comparée à un sore; elles renferment un parenchyme qui, à la germination, se gonfle et fait éclater l'enveloppe du fruit en autant de valves qu'il y a de loges. Le sporocarpe, longuement ou très brièvement pédicellé, est placé immédiatement en avant d'une feuilie; son développement n'est pas conru ; il semble cependant encore de nature foliaire. Quand la macruspore est formée, elle s'entoure d'abord d'une membrane mucilagineuse qui s'élève à son sommet en une grande papille. Sur cette première membrane se déposent deux autres couches molles à structure prismatique; toutes deux laissent au sommet un eritonnoir vide au-dessus de la papille, qui s'affaisse plus tard (Sachs).

Les sporocarpes, plus ou moins longuement pédonculés, des Marsilia prennent naissance sur la face ventrale d'un pétiole; on peut les considérer comme correspondant à des lobes foliaires; leur pédoncule est simple, à un seul fruit, ou ramifié à plusieurs fruits. Ceux-ci, en formè de haricot, se composent de deux valves cornprimées réunies 
par deux sutures ventrale et dorsale. Le pédoncule inséré à la base du fruit, y forme un raphé (Notobasalstück, M. Russow). La capsule solide et résistante est munie extérieurement de stomates. Un faisceau fibro-vasculaire s'étend le long de la suture dorsale et envoie dans les deux valves des nervures qui s'étendent jusqu'à la suture ventrale. A l'intérieur du fruit et correspondant aux sutures, se trouve un cordon annulaire corné, formé de cellules très comprimées et plus épais le long de la suture dorsale. Les sores tendus transversalement entre les deux branches du cordon auxquelles ils sont fixés, forment deux rangées longitudinales qui remplissent les deux valves; leur nombre varie de deux à douze paires.

Chaque sore correspond à une nervure et se compose d'une indusie à une seule assise de cellules et de nombreux sporanges; ceux-ci sont insérés sur un bourrelet appliqué intérieurement contre la paroi de l'indusie qui regarde la valve; ce bourrelet, longitudinal par rapport au sore et transversal par rapport à tout le fruit, chemine parallèle-ment à une nervure et porte sur sa crête les macrosporanges (12 à 280 ), à droite et à gauche les microsporanges beaucoup plus nombreux. La paroi de chaque sporange se compose encore d'une seule couche de cellules délicates, et se dissocie rapidement dans l'eau.

Dans l'état le plus jeune observé par $\mathbf{M}$. Russow, le segment foliaire destiné à former le sporocarpe, mesurait moins de 1 millimètre et était recourbé du côté du jeune pétiole, de façon à toucher presque par sa pointe le pédoncule. Sur la face ventrale se voient alors deux rangées de fossettes en entonnoir, correspondant aux sores futurs et formées en direction acropète; elles se continuent chacune par un canal transversal, le canal du sore. L'accroissement terminal du segment fertile s'arrête alors, le pédoncule au contraire s'allonge. De la nervure dorsale du segment, des cordons de procambium, les futurs faisceaux, se dirigent vers les canaux auxquels ils se superposent extérieurement. Dans chaque canal, et sur sa face la plus éloi : gnée du plan médian du fruit, on voit apparaître une série de sept à huit papilles; chacune se segmente suivant quatre faces; et bientôt, dans ces cellules terminales, une péricline sépare la cellule operculaire superficielle d'une cellule intérieure qui est l'archéspore du 
macrosporange. Les segments découpés plus bas dans la cellule terminale deviennent le point de départ du développement des microsporanges. Dans tous les sporanges il se forme seize cellules mères et soixante-quatre spores. Dans les microsporanges chaque spore devient une microspore; dans les macrosporanges, chaque tétrade développe d'abord une de ses quatre spores; mais bientôt une des seize privilégiées prend le pas sur les autres qu'elle refoule; ses trois sœurs persistent encore quelque temps. Les spores non développées et les cellules de l'endothèque se fusionnent en une épispore qui plus tard se différencie en trois couches, comme dans les Pilularia.

Le développement des sporanges diffère notablement ici de ce que nous avons vu précédemment (M. Russow).

Equisétacées (M. Duval-Jouve). - Les sporanges sont portés au sommet de branches ordinaires ou de branches spécialement transformées. A leur sommet, la cellule terminale se trouve remplacée par un méristème analogue à celui du prothalle des Fougères (M. Dutailly). Au-dessus de la dernière gaine foliaire stérile il se forme d'abord, dans ces tiges fertiles, une gaine foliaire imparfaite, l'anneau (bractées); puis on voit naître près du cône végétatif et en sẻrie acropète, des bourreiets foliaires peu. proéminents et rapprochés. La première ébauche des feuilles fertiles ressemble entièrement à celle des stériles; ce sont encore des protubérances pluricellulaires qui apparaissent à la surface de ces bourrelets. Mais bientôt une différence essentielle s'établit (M. Göbel); les rangées cellulaires médianes de la protubérance se développent plus rapidement en longueur que les rangées latérales; en même temps elles se dédoublent et se multiplient au sommet; là, elles refoulent donc en dehors et en bas les rangées périphériques qui se recourbent vers la base de la protubérance. Iue sommet de celle-ci se transforme ainsi en une tête hémisphérique donf les cellules sont régulièrement disposées en séries rayonnantes; les séries les plus extérieures sont recourbées et dirigées finalement vers la tige fertile. Sur la face inférieure de l'écusson, un certain nombre de ces séries s'accroissent plus vite que celles qui les entourent; elles formeront bientôt autant de proéminences dirigées vers la 
tige et donneront naissance $\mathfrak{d}$ autant de sporanges. Dans chacune de ces rangées, c'est encore la cellule hypodermique qui représente l'archéspore et qui produira tout le tissu sporigène. L'endothèque provient entièrement des cellules, de la paroi sporangienne comme dans les Mariattiacées et les Ophioglossées; ses cellules sont moins distinctes; car de bonne heure le tissu sporigène les comprime, ainsi que les cellules internes de l'enveloppe capsulaire, et les réduit à une bande réfringente périphérique. Les cellules mères des spores se forment comme précédemment. A la maturité, la paroi sporangienne se compose d'une seule assise de cellules; peu avant la déhiscence celles-ci acquièrent très rapidement des bandes d'épaississement spiralées sur la face dorsale, annelées sur la face ventrale du sporange (M. Duval-Jouve).

Les cellules mères des spores seraient nues d'après $\mathbf{M}$. Sachs; d'après Hofmeister et M. Russow, au contraire, elles sont toujours entourées d'une mince membrane. M. Sachs considère aussi comme formée en premier lieu et par la spore elle-niême, la membrane externe de celle-ci (élatères); tandis que Russow et Tschistiakoff la décrivent comme formée en dernier lieu et comme provenant, non de la spore, mais de l'endothèque et de la couche interne de la capsule; ce serait une épispore (pseudo-épispore, M. Tschistiakoff) analogue à celle des Marsiliacées.

Lycopodinées isosporées. - Les Lycopodium ont leurs sporanges placés à l'aisselle des feuilles; celles-ci, semblables aux feuilles ordinaires ou modifiées (bractées), sont disposées en épi terminal.

Le premier rudiment du sporange (M. Göbel, puis M. Sadebeck) apparaît de très bonne heure à la base et au côté interne de la jeune feuille. Là, plusieurs cellules, formant un groupe, s'allongent perpendiculairement à la surface foliaire; au centre de ce groupe une ou plusieurs cellules, par une cloison péricline, se divisent chacune en cellule extérieure et en cellule hypodermique qui est l'archéspore. Sur les coupes longitudinales l'archéspore se compose d'une cellule; mais ni M. Göbel, ni M, Sadebeck n'ont pu déterminer si, transver- 
salement, clle se réduisait aussi à une cellule unique ou se composait d'une rangée cellulaire.

Puis les cellules de la protubérance sporangienne voisines de la (ou des) précédente, se divisent de la même manière, mais sans que les hypodermiques qui en résultent prennent part à la formation de - l'archéspore. Toutes les cellules extérieures se multiplient par cloisons longitudinales; en même temps le jeune sporange se soulève davantage; ensuite, par une péricline, elles donnent naissance à deux couches dont l'externe ne se segmente plus que perpendiculairement à la surface, tandis que l'interne se divise en deux assises; l'assise intéricure forme l'endothèque dont les cellules, plus riches en protoplasma, s'arrondissent; à la base des sporanges l'enáothèque est aussi fourni par les cellules adjacentes et non par l'archéspore. Celleci, pendant ce temps, s'est divisée assez régulièrement par des cloisons rectangulaires et a donné naissance finalement aux cellules mères des spores qui produisent toujours chacune quatre spores. Tout l'organe alors a augmenté de volume, est devenu réniforme, et s'est soulevé sur un court pédicelle. A la maturité l'enveloppe de la capsule se déchire du sommet à la base.

Le Phylloglossum Drummondi possède des épis courts, ovales, analogues à ceux des Lycopodium; ils ne portent de sporanges que sur leurs bractées inférieures.

Des opinions diverses ont été émises sur les sporanges de Psilotum placés au nombre de trois ordinairement sur un petit rameau qui porte encore, sous les sporanges, deux petites feuilles. Pour MM. Sachs at Strasburger, ce rameau est l'homologue d'un épi fertile de Lycopode qui commencerait par deux feuilles stériles, et se réduirait ensuite à trois sporanges plongés dans l'axe. M. Juranyi considère le tout comme une branche latérale métamorphosẻe. D'après MM. Kickx et Luerssen, les sporanges sont produits par la base de la feuille bipartite placée au-dessous; leur formation serait analogue à celle des sporanges de Marattia. D'après M. Prantl on a affaire à un sore composé ordinairement de trois, quelquefois de deux ou quatre sporanges. Le sujct a été repris récemment par $M$. Göbel. D’après cet auteur, 
on pourrait considérer le petit groupe comme un sporange à trois loges; ce groupe n'est pas une émergence de la base de la feuille, mais une formation sui generis, comme les sporanges en général, qu'il ne faut pas s'évertuer à ramener partout à des émergences foliaires. Le premier rudiment du rameau sporangifère ressemble entièrement à celui d'une branche ordinaire résultant d'une ramification monopodique; il est dépourvu de cellule terminale et s`infléchit vers le sommet de l'axe dont il émane; au-dessous de sa pointe terminale, mais tout près d'elle, naissent deux proéminences, premières ébauches des feuilies; elles se rapprochent ultérieurement sur la face extérieure du rameau. La portion du rameau placée au-dessus de ces feuilles, constitue le pédicelle, ordinairement peu développé, de tout le groupe sporangien; vers son sommet prennent naissance trois ou quatre sporanges. Mais vers le point d'insertion des feuilles, il se forme sur le rameau un bourrelet proéminent qui se développe en une demi-gaine aplatie, portant les deux folioles, et formant avec elles la feuille bifide. Il arrive que le sommet du support sporangien avorte et se réduit à une petite protubérance placée entre les deux feuilles; d'autres fois ce sommet se développe en une pointe verte recourbée. Quelquefois aussi l'une des deux feuilles devient bifide, et l'on croit avoir affaire à une feuille unique trifide. Les sporanges ne se forment pas au point végétatif mème du support, mais un peu au-dessous de ce point dont l'accroissement alors s'arrête bientôt. Chaque archéspore se réduit primitivement à une cellule unique; elles sont séparées l'une de l'autre par quelques rangées de cellules. C'est le tissu sporigène qui produit ici l'endothèque. Sur la ligne médiane de chaque sporange, les cellules de la paroi externe éprouvent un accroissement moindre, et constituent ainsi une bande marquant la déhiscence; mais les sporanges eux-mêmes ne sont nullement biloculaires.

Le genre Imesipteris présente des feuilles plus grandes, munies chacune d'un faisceau médian; les sporanges, réunis par deux seulement́, sont portés sur un pédicelle d'un demi-centimètre de longueur. Le rameau ou support sporangien prend naissance encore près du sommet végétatif de la tige et s'inc'urve vers ce sommet. Les deux sporanges sont placés d aborif près de l'extrémité de leur sup- 
port, l'un du côté de la tige, l'autre du côté opposé; mais par suite d'un accroissement inégal de ce support, dans. sa portion comprise entre les feuilles et les sporanges, ceux-ci se trouvent plus tard tour. nés tous deux du côté de la tige.

Dans les deux genres précédents (famille des Psilotacées), les sporanges ne sont donc pas des émergences de feuille, mais sont produits par de courts axes latéraux, dans le tissu desquels ils restent plongés. Cela les éloigne des Lycopodium et les rapproche jusqu'à un certain point des Selaginella. M. Göbel compare ces groupes sporangiens aux groupes de macrosporanges de certaines Conifères, du Ginkgo par exemple, et fait remarquer que, dans cette classe, on peut, dans un même genre, truuver des macrosporanges et des microsporanges formés très différemment : les uns axillaires, les autres foliaires.

Lycopodinées hétérosporées. - Dans les Selaginella les fe uilles fertiles, ordinairement un peu différentes des stériles, forment un épi quadrangulaire terminal. Un ou plusieurs macrosporanges sont disposés sur les feuilles inférieures de l'épi, quelquefois en rangée verticale; ils renferment le plus souvent quatre, rarement deux ou huit macrospores. Les microspores sont très nombreusés. Le pédicelle est to ujours court, épais, plurisérié; la paroi capsulaire fossède deux assises. Les sporanges sont insérés sur l'axe un peu au-dessus de la feuille, rarement à l'aisselle de celle-ci. Ils ont été considérés comme appartenant à la feuille (MM. Hegelmaier, Strasburger); ils naîtraient, en effet, comme formation nouvelle, sur la feuille à peine ébauchée et se trouveraient déplacés ultérieurement, pendant l'allongement du cône végétatif. Cette opinion se trouve contredite par les récents travaux de M. Göbel; le rudiment du sporange est une émeronce, non pas de l'ébauche foliaire, mais de la tige elle-même; il prend naissance tout près du point végétatif aux dépens des cellules superficielles placées immédiatement au-dessus de la protubérance foliaire. Le développement est ensuite analogue à ce que nous avons vu dans les Lycopodium, sauf en un point: l'endothèque est formée par l'archéspore elle-mème, dans la portion supérieure du sporange; dans sa portion basilaire, c'est, .comme dans les Lycopodium, le tissu adja- 
cent qui le constitue. L'endothèque a donc ici absolument la même origine que dans l'anthère des Angiospermes, où il provient aussi (de mème que le mésothèque) de lá couche hypodermique corticale dans toute la portion externe de l'anthère; mais où il est fourni également par le tissu adjacent dans lá portion basilaire de l'anthère, c'est-à-dire dans celle qui regarde le connectif. Jusqu'à la formation des cellules mères des spores les macro et microsporanges se ressemblent entièrement; dans ces derniers elles donnent toutes des tétrades; dans les premiers vne seule cellule mère donne quatre macrospores; les autres ne se divisent pas et ne tardent pas à être résorbées. La déhiscence se fait comme dans les Lycopodium.

Les microsporanges des Isoetes se trouvent à la base des feuilles internes, les macrosporanges à la base des feuilles externes dans les rosettes fertiles. I.es uns et les autres sont insérés sur un très court et large pédicelle dans la fossette de la gaine foliaire, et divisés en loges incomplètes par des trabécules tendues de leur face ventrale à leur face dorsale. Leur premier rudiment dejà se compose d'un groupe de cellules (MM. Hegelmaier, Göbel), et tout le tissu sporigène provient d'une couche de cellules hypodermiques distinctes par leur abondant plasma. Au moment où ce tissu (archéspore) apparaît, la lèvre est déjà distincte et la ligule représentée au-dessus d'elle par une petite masse cellulaire. Le développement est différent pour les macro et les microsporanges.

Dans les derniers, les cellules de l'archéspore s'allongent perpendiculairement à la surface de l'organe, et, par une série de cloisons transversales, donnent naissance chacune à une rangée cellulaire; certaines de ces rangées deviennent muins riches en protoplasma, se divisent moins activement et allongent leurs cellules; ce sont les parties stériles du sporange, les trabécules, où apparaissent plus tard dés lacunes aériennes. Les autres rangées constituent le tissu fertile ou sporigène; elles forment de bonne heure, vers la surface du sporange, une ou plusieurs cellules de tapis qui se multiplient par bipartitions; mais la majeure partie de la rangée donne naissance, par de nombreuses divisions, aux cellules mères des spores. Les trabécules et le pédicelle produisent l'endothèque sur les faces latérales et postérieure de chacun des groupes fertiles qui s'en trouvent alors enve- 
loppés complètement. Le tissu de la feuille s'accroît plus vite tout autour du sporange qui se troure ainsi placé dans une fossette; le velum ou indusie se développe ensuite. Les cellules mères forment ici leurs spores, non par quadripartition presque simultanée, mais par deux bipartitions successives, comme dans les Monocotylédones.

Dans les macrosporanges les trabécules se constituent encore d'une façon analogue. Les cellules fertiles de l'archéspore donnent aussi naissance chacune, par plusieurs périclines, à une rangée de plusieurs cellules, rangée perpendiculaire à la surface de l'organe: la cellule interne de la série devient directement une cellule mère unique de spores; les autres seront des cellules de l'endothèque qui sera complété, comme précédemment, par les trabécules et le pédicelle. Bientôt les cellules mères arrondissent leurs angles et augmentent de volume, refoulant leurs voisines. Les cellules de l'endothèque se sont isolées, elles se trouvent alors résorỉées en direction centrifuge et les cellules mères deviennent libres, chacune dans sa cavité; elles augmentent encore de volume, puis se divisent en quatre, mais d'une façon particulière (M. Strasburger) : la préparation au cloisonnement prècède la première division du noyau, et les systèmes lenticulaires de filets protoplasmiques sont déjà constitués, reliant entre elles quatre masses condensées de protoplasma, avant que le noyau latéral ait commencé à se diviser. Il se segmente ensuite à deux reprises, et chacun des quatre nouveaux noyaux pénètre dans une des quatre masses plasmiques. A ce moment, les lames granuleuses, puis les cloisons se forment simultanément pour les quatre cellules. Les quatre nouveaux noyaux ne se forment donc pas indépendamment de l'ancien, comme on l'avait cru d'abord.

Ce qui précède montre que, chez les Cryptogames vasculaires, 'le sporange est loin d'avoir toujours la même valeur morphologique, et que son développement n'est pas non plus uniforme. Cependant nous devons signaler quelques caractères communs très importants, et d'aburd la formation endogène de l'archéspore.

Nous avons vu, en effet, que le tissu sporigène peut se ramener constamment à une ou plusieurs cellules hypodermiques ou apparte- 
nant à la couche extérieure du tissu fondamental; il ne prend pas naissance différemment chez les Phanérogames. L'existence transitoire d'un tapis ou endothèque est aussi générale; son mode de formation, variable chez les différentes Cryptogames vasculaires, se retrouve presque identique dans les sporanges des Lycopodinées hétérosporées, d'un côté, et dans les microsporanges des Phanérogames, de l'autre. Le développement des sporanges se termine toujours par la quadripartition des cellules mères, et ce caractère, que nous avons rencontré chez les Muscinées, se retrouve encore dans les microsporanges des groupes supérieurs, dont les macrosporanges ne le présentent plus. Il n'est pas inutile de rappeler que déjà, chez les Cryptogames hétérosporées, cette quadripartition tend à s'altérer, et que, chez les Rhizocarpées, la cellule mère ne donne naissance, en fait, qu'à une seule spore. Que sous l'influence de l'hérédité ce processus se simplifie de manière que le résultat se trouve atteint plus vite, et alors par une espèce de condensation, la cellule mère se transformera directement en une spore unique. Supposons une pareille condensation réalisée dans le macrosporange d'une Isoète, chez laquelle une seule cellule de l'archéspore serait fertile, toutes les autres ne donnant que des trabécules, et nous aurons le nucelle et la cellule mère du sac d'une Phanérogame.

La spore est le dernier terme dans le développement des sporanges et de la plante asexuée; elle sert en même temps de point de départ à la nouvelle génération. Dans toutes les Isosporées, elle donne, en germant, un prothalle à nutrition indépendante (comme dans les Mousses), qui ne formera les organes sexuels qu'après un certain degré de développement végétatif; ce prothalle porte généralement les deux sexes. Dans les Hétérosporées, au contraire, le prothalle montre une dioecie complète, et se réduit en même temps à une émanation toujours plus faible de la spore qui lui fournit toute sa subsistance; les spores, elles-mêmes, présentent déjà, entre elles, des différences correspondantes aux sexes. Les organes agames, qui constituent le trait d'union entre les deux générations, se ressentent donc déjà de la sexualité. Sous cette influence, le développement des grandes spores femelles éprouve de notables modifications : réduction du nombre 
des cellules mères, avortement d'une partie de celles-ci, avortement de trois spores dans une tétrade. La simplification, dans ce sens, va s'accentuant dans les Phanérogames; elles peuvent encore posséder plusieurs cellules mères dans un sporange, comme dans les Rosacées par exemple (MM. Strasburger, Fischer, Guignard) ; mais ordinairement il n'y a plus qu'une seule cellule mère, se changeant en macrospore, sans quadripartition. Celle-ci ne s'échappe plus du macrosporange, dont le tissu, en grande partie stérile, sert à sa nutrition, puis à celle de l'embryon; la réserve nutritive passera donc en partie de la spore dans le sporange; si le volume de la première devait augmenter dans les Hétérosporées, il diminuera de nouveau dans les Phanérogames. Le macrosporange, à son tour, reste fixé sur le sporogone; dans le genre Ginkgo, il en est encore séparé au moment de la fécondation par une assise lignifiée; mais il reste, dans la plupart des Phanérogames, en connexion intime avec la plante asexuée, même après la fécondation; il pourra donc tirer des matières nutritives du sporogone, et les transmettre à l'embryon, qui ne se détachera que muni des réserves nécessaires à son premier développement.

La genèse des petites spores n'est pas modifiée sensiblement par la sexualité qui les atteint; elle ne diffère pas très notablement des Hétérosporées aux Isospcrées, ni même de celles-ci aux Mousses; elle se retrouve encore essentiellement la même dans les Phanérogames. Les sporanges, dans ce vaste groupe, sont aussi d'abord très reconnaissa bles; dans les Cycadées, ils sont encore réunis en sore et placés sur un coussinet, qui rappelle celui des Fougères (M. Warming); ils se transforment ensuite plus profondément; le mésothèque assurera mieux leur déhiscence (M. A. Chatin); les feuilles qui les portent, réalisent ensuite des modifications favorables à la dispersion du pollen par le vent ou par les insectes, et conséquemment favorables aussi à la fécondation. Des émergences foliaires (homologues peut-être de l'indusie ou du velum), des feuilles entières même s'adaptent spécialement, d'un autre côté, de façon à protéger et à nourrir le macrosporange (nucelle) et sa spore, à assurer la pénétration jusqu'à l'œuf de la génération mâle, à abriter et à nourrir le jeune embryon. La sexualité atteint donc de plus en plus profondément la génération agame. Aux 


\section{$-157-$}

étamines, téguments séminaux et carpelles qui ont ainsi pris naissance, se joignent encore finalement les feuilles situées plus loin sur l'axe, et cet axe lui-même; ces organes se modifient pour attirer les insectes, et pour faire contribuer les oiseaux, les mammifères, à la dissémination, etc. C'est par suite de ces transformations successives, en sens divers, mais toutes corrélatives d'avantages réalisés par lá descendance, qu'ont dû apparaître peu à peu la fleur et le fruit.

\section{LES SPORES ET LEUR GERMINATION.}

Quand la cellule mère s'est divisée en quatre, chaque spore s'entoure d'une nouvelle enveloppe dans sa cellule mère spéciale; puis, les parois de celle-ci et de la première cellule mère étant résorbées, les spores s'isolent dans l'intérieur du sporange, dont la déhiscence les rend libres.

Si la cellule mère a subi deux bipartitions répétées, elles sont tout d'abord disposées comme les quatre quartiers d'une sphère, et elles conservent plus tard une forme correspondante, présentant, d'un còté, entre deux surfaces planes, une arrête saillante qui est en même temps la ligne de déhiscence (Polypodium, Aspidium); ces spores sont bilatérales.

Dans la quadripartition simultanée, les spores sont disposées en tètrades, et chacune offre, sur une de ses faces, trois arêtes saillantes qui marquent encore les lignes de déhiscence; elles sont alors radiales (Hyménophyllacées, Ophioglossées). Dans l'un et l'autre cas, elles arrondissent plus ou moins leurs angles et leurs arêtes, ultérieurement à leur formation. Cette différence n'est pas essentielle; car on peut rencontrer les deux espèces de spores dans la même famille ( $\mathrm{Po}-$ lypodiacées, Gleichenaciées), dans la même espèce (Lycopodium inundatum, etc.) et jusque dans le même sporange.

Toujours très réduites, les dimensions des spores varient entre 27 et 158 millièmes de millimètre dans les Isosporées ; dans les Hétérosporées, les macrospores atteignent de tout autres dimensions. 
Leur enveloppe se compose ordinairement de trois couches (M. Tschistiakoff): $1^{\circ}$ L'endospore, difficile à distinguer du contenu de la spore, présente presque toujours la réaction de la cellulose, et était considérée comme formant l'enveloppe du premier filament, issu de la spore; mais d'après un récent travail de M. Rauwenhoff, le protoplasma sécrète, en réalité, à ce moment, une membrane nouvelle, et aucune des couches de l'enveloppe de la spore ne passe dans la jeune plantule.

$2^{\circ}$ L'exospore est une membrane de protection, qui se trouve rompue à la germination; elle se compose de deux couches, et, quand l'épispore manque, sa surface est marquée de creux et de protubérances ; elle ne présente pas la réaction de la cellulose.

$3^{\circ} \mathrm{L}^{\prime}$ épispore est brune, plissée et se détache facilement; elle semble avoir passé souvent inaperçue; suivant son origine, M. Tschistiakoff la distingue en périspore ou épispore proprement dite, quand elle provient de la membrane de la cellule mère spéciale (Polypodiacées), ct en pserido-épispore, quand elle se forme aux dépens de la masse plasmique qui entoure les cellules noires spéciales (pseudo-épiplasma); cette dernière variété se rencontre presque exclusivernent dans les macro et microspores des Hétérosporées. L'épispore peut faire complètement défaut (Lycopodium alpinum).

Les spores des Equisetum présentent une particularité remarquable; leur épispore se compose de deux rubans spiralés hygroscopiques qui se déroulent par la dessiccation et s'enroulent de nouveau dans une atmosphère humide; quand les mouvements de ces élatères se succèdent rapidement (insufflation de l'haleine), les spores sautillent vivement. Ces mouvements facilitent peut-être la sortie des spores de leurs sporanges; mais ensuite ils semblent avoir pour effet, non pas de disperser les spores isolément, mais de les tenir au contraire réunies par petits groupes, les élatères s'accrochant et s'enchevêtrant entre eux. Cette disposition toute spéciale coïncide avec une tendance à la diœcie chez les prothalles provenant de ces spores; elle aurait donc de l'importance pour la reproduction.

Qund les spores sont placées dans des conditions favorables, leur contenu se gonfle et la membrane externe éclate laissant sortir le pro- 
toplasma cntouré d'une membrane de cellulose. Cette rupture a lieu ordinairement le long des arêtes de déhiscence, auxquelles correspondent dans l'exospore soit des gouttières, soit des bourrelets. Dans les Equisétacées l'exospore se brise en deux valves, sansque le cercle de déhiscence soit indiqué d'avance. Au moment où le protoplasma de la spore devient actif, la matière verte, entourant jusque-là le noyau comme une masse nébuleuse, se dispose en corps chlorophylliens (Osmundacées) ; ce changement ne peut s'observer évidemment dans l'intérieur de la spore que quand l'exospore est transparente (Equisétacées, Hyménophyllacées, Osmundacées); il n'a lieu quelquefois qu'après la rupture de l'exospore (Marattiacées, par exemple).

A la germination, le protoplasma se constitue une membrane nouvelle indépendante des anciennes enveloppes de la spore; appliquée d'abord contre celles-ci, elle s'accroît rapidement à la déhiscence et entoure la papille qui apparaît alors au dehors ; cette formation nouvelle a été observée tout d'abord sur les spores peu nombreuses dont l'enveloppe interne (endospore) n'est pas de la cellulose, c'est-à-dire ne se colore pas en bleu par l'iode et l'acide sulfurique (Osmunda, Gleichenia); mais elle a été constatée aussi dans d'autres cas, par exemple, dans les microspores des Marsiliacées, les zygospores des Conjuguées. La cellule primitive constituée par la formation de cette membrane, commence quelquefois sa segmentation encore contenuc dans l'enveloppe de la spore, celle-ci étant déjà rompue (Angiopteris, Marattia) ou même encore entière (Trichomanes, Gleichenia); son développement ultérieur donne naissance au prothalle.

Les premiers indices de la germination s'observent un temps trèsvariable après ensemencement des spores mûres; il est, par exemple, de vingt-quatre heures pour les Equisétacées, de cinq à six jours pour les Angiopteris. Certaines spores sont mûres à la déhiscence du sporange; ce sont toujours des spores vertes qui alors peuvent ne conserver que quelques jours le ur faculté germinative (Equisetum, Osmunda, par exemple). D'autres ne deviennent mùres qu'après une période de repos assez longue, à la suite de laquelle seulement elles sont susceptibles de former de la chlorophylle. Il y a des états intermédiaires: 
les spores de Lycopodium inundatum peuvent se développer à la sortie du sporange, mais leur faculté germinative est beaucoup augmentée au printemps suivant. On a vu cette faculté se conserver dix à vingt ans pour les spores de certaines espèces :

On a pu faire germer dans l'obscurité les spores de Pteris, Aspi. dium, Equisetum, etc. ; mais c'est un prothalle anormal qui so développe dans ces conditions; il porte quelquefois des anthéridies, jamais des archégones. La lumière est indispensable pour la formation normale du prothalle, peu importe qu'elle soit directe ou diffuse. La première tend seulement à supprimer la phase protonéma (1) (filament). Quand la lumière et l'espace font défaut, il ne se forme au contraire qu'un filament pluri-cellulaire ou même un long filament uni-cellulaire. Une certaine température est aussi nécessaire à lá germination ; cette condition est très variable avec l'habitat géopraphique de la plante. Enfin, la germination ne peut se faire que dans une atmosphère humide et sur un substratum imprégné d'eau ; celle-ci pénètre jusqu'au protoplasma, grâce aux pores et aux fentes (lignes de déhis. cence) qui existent naturellement dans l'exospore.

\section{GÉNÉRATION SEXUÉE}

\section{PROTHALLE DES ISOSPORÉES.}

Filicinées. - La cellule primordiale de la spore engendre souvent dans les Hyménophyllacées un véritable protonéma; plusieurs filaments en dérivent qui se ramifient, forment des poils rhizoïdes; donnent naissance latéralement à des prothalles lamelliformes bientôt indépendants, et développent, comme organes de propagation sans doute, des cellules en forme de bouteille. Tout ceci rappelle beaucoup les Muscinées. D'autres fois un seul des filaments primitivement for-

(1) Je préfère employer ici, commo pour les Mousses, le mot de protonema, celui de proembryon ne me paraissant pas tres juste et pouvant, d'ailleurs, causer une confusion avec le suspenseur. 
més, prend bientòt la prépondérance et puurra se transformer directement $\in \mathbf{n}$ lame foliaire, ce qui constitue une réduction du protonérna. Chez les Polypodiacées celui-ci ne consiste plus qu'en un filament court et articulé; à son extiémité se montre une cellule terminale à deux faces qui ne tarde pas, en se segmentant, à se trouver placée au fond d'un angle rentrant. Bientòt aussi cette cellule se transforme en un méristème marginal qui continue à développer une lame cellulaire en forme de cœur. Plus tard seulement, dans ce méristème, apparaissent des cloisons paralièles à la surface du prothalle, produisant un coussinet à plusieurs assises (M. Prantl). Dans quelques espèces le méristème ne se forme pas aux dépens de la cellule terminale axıle, mais apparait latéralement; néanmoins il remplace cette cellule dans la production du prothalle; seulement les lobes de ce prothalle secondaire ne correspondront pas à ceux du prothalle primitif (Gymnogramme leptophylla). Enfin, dans d'autres especes, le prothalle peut devoir son origine tantôt à l'un, tantôt à l'autre mode (Pteris, Aspidium, etc.).

Le prothalle porte à sa face inférieure des poils rhizoïdes unicellulaires, des anthéridies qui ont la même valeur morphologique et qui peuvent être engendrés, comme ces poils, par toute cellule du prothalle. Les archégones, d'une valeur morphologique plus élevée, dépendent directement du méristème; ils n'apparaissent jamais que sur le coussinet et en direction acropète. Souvent on voit prendre naissance des prothalles anormaux, dépourvus de méristème (améristiques, M. Prantl); ils sont plus ou moins réduits et ne donnent point d'archégones; même composés seulement de quelques cellules, ils forment encore des anthéridies (MM. Cornu, Nägeli, Schacht). M. Borodin a observé un prothalle unicellulaire portant trois anthéridies (Allosurus sagittatus).

Les Gleicheniacées (M. Rauwenhoff) forment, en géréral, leur prothalle comme la famille précédente. Les anthéridies apparaissent après quatre mois, les archégones après six; les premières ne sont pas marginales, et se forment ordinairement à la face inférieure, quelquefois à la face supérieure du prothalle; leur nombre est consi- 
dérable. Fréquemment la spore produit directement une petite masse cellulaire (coussinet).

Dans les Osmundacées la papille, apparaissant à la germination, devient le premier rhizoïde qu'une cloison sépare du reste de la spore, laquelle se divise en quatre quartiers; l'un des quartiers antéricurs devient la cellule terminale; bientôt encore l'accroissement n'est plus que marginal. Souvent, dans les Todea, il se forme d'abord un protonéma pluricellulaire, quelquefois aussi directement une masse cellulaire (Todea, Osmunda). Le coussinet constitue ordinairement dans cette famille une nervure médiane portant de nombreux archégones. S'ils ne sont pas fécondés, la nervure forme un lobe proéminent dans l'angle rentrant du prothalle. Celui-ci se ramifie, mais les bourgeons adventifs qu'il forme ne semblent pas devenir indépendants.

Comme la famille précédente, les Marattiacées (MM. Jonkman, Luerssen) possèdent une lame prothallienne ou une masse cellulaire. Dans le premier cas la cellule primitive agrandie se divise en quatre directement; le coussinet plus développé que précédemrnent apparaît de bonne heure, les rhizoïdes au contraire tardivement. Dans le second cas la germination se fait tout d'abord comme dans les Osmundes, mais les quartiers se divisent en octants, dont les quatre inférieurs deviennent encore des rhizoïdes; un des supérieurs se change cn cellule terminale, et produit le prothalle, dont l'accroissement ne tarde pas à devenir marginal. Les anthéridies sont enfoncées dans le prothalle, et nous avons ici, d'une façon générale, une transition vers les Ophioglossées. Le défaut de lumière eí d'espace peut déterminer la formation d'un filament (protonéma). Les pousses marginales deviennent indépendantes.

Le prothalle des Ophioglossées (Hofmeister, Mettenius) est une masse cellulaire souterraine, à développement encore inconnu. A la face supérieure archégones et anthéridies sont enfoncés dans le tissu. Peutêtre cette masse ne représente-elie que le coussinet d'un prothalle ordinaire qui se formerait comme dans le cas suivant.

Nous n'avons pas en effet, dans les Polypodiacées, signalé le déve- 
loppement singulier du Gymnogramme lepthophylla (M. Göbel). Là le coussinet se transforme en une masse cellulaire ou pousse (tubercule) fertile qui s'enfonce dans le sol, perd sa chlorophylle, mais se remplit d'amidon et de graisse. Le prothalle portant des anthéridies dans le voisinage du coussinet, se flétrit, souvent seulenient après production de pousses marginales qui ne portent que des anthéridies ou qui reproduisent d'autres prothalles et finalement d'autres pous. ses. Le tubercule porte des rhizoïdes à sa face inférieure, des archégones à sa face supérieure. Si ceux-ci ne sont pas fécondés le tubercule, après un temps de repos, développe de nouveau deux lobes de prothalle, entre lesquels un nouveau méristème donne une nouvelle pousse fertile. Cette propagation végétative de la génération sexuée a une grande importance pour le Gymnogramme leptophylla qui est. annuel.

Équisétacées. - La première cloison se forme à l'intérieur de la spore; la papille se transforme en rhizoïde; la cellule qui reste dans la spore se divise par une cloison perpendiculaire à la première en deux (quelquefois aussi en trois) cellules juxtaposées dont chacume s'allonge en un filament cloisonné; ceux-ci restent quelquefois plus ou moins soudés à leur base. La cellule terminale de chaque filament se divise bientôt de nouveau par une paroi longitudinale; alors il peut y avoir nouvelle bifurcation ou formation d'une lame. Ici plus que partout ailleurs, les cloisons se rencontrent entre elles à angle droit. La formation des anthérides est basipète, celle des archégones acropète et liée au méristème qui ne se constitue que tardivement. Tout d'abord en effet la lame prothallienne s'accroit par toute sa périphérie et se ramifie beaucoup; les rameaux peuvent devenir indépendants. Dans les prothalles mâles un de ces lobes devient prépondérantacquiert plusieurs assises de cellules et forme les anthéridies. Dans un même semis les archégones, placés à la face supérieure sur un cousṣinet, n'apparaissent sur les prothalles femelles, bien plus vigoureux, que plusieurs semaines après la formation des anthéridies dont les prothalles restent beaucoup plus petits. Il n'y a que de rares exceptions à la dinecie; elles ont lieu par la formation d'anthéridies sur les 
rameaux du prothalle femelle dont les archégones meurent alors. A l’ombre les prothalles se réduisent à des filaments.

Lycopodiacées. - M. de Bary a seuul réussi à faire développer les spores. La germination se fait sous terre; la cellule primitive se segmente en deux, dont l'une, la basilaire, reste indivise; l'autre devient une cellule terminale à deux faces. Les segments se divisent chacun en deux par une closion tangentielle; les cellules périphériques contiennent de la chlorohylle, les centrales en manquent. Les prothalles les plus développés, obtenus par M. de Bary, étaient de onze cellules. Dans la nature ils n’ont été encure rencontrés que par M. Fankhauser.Ils sont souterrains, tuberculeux, d'un blanc jaunàtre, sans chlorophylle, ni amidon. Quelques rhizoïdes sur la face inférieure lisse, anthéridies et archégones plongés dans le tissu de la face superieure qui est marquẻe de sillons et de bourrelets.

\section{ANTHÉRIDIES DES ISOSPORÉES}

Les anthéridies paraissent aussi avoir la valeur morphologique de trichomes chez les Polypodiacées et être de formation endogène dans les Marattiacées et Lycopodiacées. Elles se développent avant les archégones, sur des prothalles souvent très jeunes, quelquefois encore filamenteux et même unicelluglaires. Dans les Aneimia, par exemple, leur première ébauche se montre sous forme d'une protubérance à la face inférieure du prothalle; cette protubérance ne diffère que jar sa largeur plus grande du rudiment d un poil ; une cloison transversale la sépare de la cellule qui l'a formée; une deuxième cloison parallèle la première, sépare de cette cellule mère de l'anthéridie une cellule basilaire aplatie en disque. Une cloison courbe vient s'appliquer sur ce disque comme un verre de montre; elle découpe la ceilule supérieure en une cellule périphérique ayant la forme d'une cloche renversée et une cellule centrale coiffée par la première. Ces deux éléments augmentent simultanément de volume, puis l'extérieur se trouve divisé par une cloison en forme d'entonnoir en une cellule 
supérieure operculaire (tronc de cone renversé) et une cellule inferieure annulaire. Toute l'anthéridie s'allonge; la cellule centrale, par bipartitions repétées, se divise en un petit nombre de cellules mères d'anthérozoîdes à paroi mince et à plasma dense.

Dans les Polypodiacées les anthéridies se forment souvent d'une façon analugue; il peut y avoir deux cellules annulaires et alors la celiule basilaire disparaît ordinairement (Pteris serrulata, la plupart des Asplenium), ou trois annulaires; les cloisons peuvent se succéder dans un autre ordre, l'annulaire étant séparée d'abord d'une cellule mediane qui se divise ensuite en operculaire et en centrale (Asplenium alatum). L'anthéridie peut s'enfoncer dans le prothalle et n'être plus que très peu proéminente.

Dans les Osmuruclacées les cellules annulaires incomplètes empiètent l'une sur l'autre et se touchent obliquement (Luerssen).

Celles des Hyménophyllacées analoggres aux précédentes sont soulevées par l'allongement de la cellule du prothalle qui les porte. (MM. Janczewski et Rostafinski, Mettenius.)

Les Marattiacées ont les leurs plongées dans le tissu à la face supérieure ou inférieure du prothalle, jamais sur les bords ;'elles ne proéminent point. Une cellule superficielle du prothalle se segmente, par une péricline, en cellule centrale qui se divisera en cellules mères, et en cellule operculaire. Celle-ci, par trois anticlines, fournit trois cellules périphériques très inégales, qui ordinairement se subdivisent encore irrégulièrement, et une cellule médiane qui se trouve rompue pour la sortie des anthérozoïdes (Luerssen, Jonkman).

La cavité de l'anthéridie des Ophioglossées est plongée dans le tissu du prothalle à sa face supérieure; elle communique par une étroite ouverture avec l'extérieur, elle n'est séparée de la surface que par une ou deux assises de cellules.

Les Lycopodiacées, peu connues, semblent analogues aux précédentes.

Lianthéridie des Equisétacées paraît encore provenir d'une cellule inère unique; elle peut être terminale ou latérale sur la pousse principale ou sur une ramification (M. Sadebeck). Cette cellule superficielle par quatre cloisons perpendiculaires entre elles et à la surface, se 
divise en quatre cellules périphériques et une médiane enveloppée par les premières; dans celle-ci apparaît une paroi (quelquefois deux) parallèle à la surface; il se forme ainsi une operculaire et une celiule centrale; celle-ci, par des cloisons dans les trois directions, se divise en de nombreuses cellules mères d'anthérozoïdes. En mème temps toute l'anthéridie s'allonge et devient proéminente à la surface. L'operculaire, par deux cloisons perpendiculaires, se divise en 4 cellules qui s'écartent à la maturité pour laisser échapper les anthérozoïdes. Rarement une pousse ne produit qu'une seule anthéridie; mais plusieurs se forment ordinairement en direction basipète et sont ảisposées alors comme aux angles d'un triangle. Cette anthéridie ne semble pas avoir la valeur' morphologique d'un trichome (M. Sadebeck).

Chaque cellule mère donn€ un seul an thérozoïde. Elle posséde d'abord un noyaı qui ensuite disparaît; en même temps la couche externe de la membrane cellulaire se gélifie, enveloppant d'un mucilage la cellule qui devient sphérique, et qui s'isole alors de ses congénères; son protoplasma s'accumule le long des parois et se dispose ensuite en une spirale, laissant au centre une vacuole avec plasma granuleux et grains d'amidon; cette vacuole est entourée d'une très mince membrane et forme la vésicule que l'anthérozoïde entraîne avec lui. La cellule mère s'agrandit notablement, sa membrane se gélifie aussi sur sa face interne. Les mucilages interne et externe des cellules mères se gonflent, rompent l'opercule de l'anthéridie, dont tout le contenu s'échappe alors. La résorption de ce qui reste de la paroi des cellules mères met les anthérozoïdes en liberté, et ceci a lieu tantôt à l'Extérieur, tantôt à l'intérieur de l'anthéridie. L'anthérozoïde contourné en spirale se meut très vite à l'aide des cils qui garnissent sa région antérieure et que l'on distingue quelquefois déjà à l'intérieur de la cellule mère; au bout d'une demi-heure à peu près les mouvements se ralentissent. Le corps de l'anthérozoïde est homogène, ainsi que les cils. Le vésicule a toujours disparu au moment de la fécondation; elle ne constitue done pas la partie essentielle de l'élément fécondant, comme le prétendait Schacht.

Il est, au contraire, probable que les résultats obtenus par M. Schmitz, pour les Characées, doivent encore être appliqués ici, et 
que c'est le noyau qui forme la partie essentielle, le corps de l'anthérozoïde, le protoplasma de la cellule ne fournissant que les cils.

Les anthérozoïdes peuvent varier par la taille, par le nombre de leurs tours de spire, par la quantité de cils, etc.

\section{ARCHEGONES DES ISOSPOREES.}

De même que les anthéridies, ils présentent une grande analogie avec les Mousses; on y distingue toujours un col et un ventre, et dans celui-ci l'oosphère. Le col, à quatre rangées de cellules, enveloppe le canaỉ composé de deux cellules : cellule du col du canal et cellule ventrale du canal. Ces parties essentielles se retrouvent avec une uniformité remarquable (Janczewski, Strasburger, Kny, Sachs) depuis les Muscinées jusqu'aux Gymnospermes; cependant l'ensemble de l'organe subit une réduction croissante, et dans les Cryptogames vasculaires inférieures, i] est déjà moins développé que chez les Mousses.

L'archégone est placé chez les Fougères à la face inférieure du pro-thalle. Une cellule superfícielle peu proéminente du coussinet, se divise successivement par deux cloisons transversales en une rangée de trois cellules; ce sont: la cellule basilaire, la cellule mère de la rangée centrale et la cellule mère de la périphérie du col. La première est sans importance dans le développement ultérieur (M. Janczewski); d'abord riche en protoplasma et pauvre en chlorophylle comme ses deux cellules sœurs, elle est très distincte des cellules voisines du prothalle; mais elle ne tarde pas à devenir verte comme celles-ci, à se segmenter et à se confondre avec le tissu prothallien. La dernière cellule (périphérie du col) est coupeje, par deux cloisons perpendiculaires entre elles et à la surface, en quatre cellules disposées en croix qui, se divisant à leur tour par des cloisons assez obliques, mais narallèles d'une façon générale à la surface, deviennent les quatre rangées du col, rangées composées d'un nombre de cellules variable avec les familles. C'est ainsi que, dans les Polypodiacées, le col, recourbé vers la région postérieure du prothalle, compte six cellules dans sa partic convexe et 
quatre dans la concare, que dans les Osmundacées et Hyménophylla. cées, où le col est drnit, leur nombre est de quatre.

Pendant cette multiplication, la cellule mère de la rangée centrale insinue éntre les quatre séries du col son extrémité supérieure pointuie qu'une cloison sépare de la partie large inférieure de la cellule; cette pointe s'allonge dans le col et en remplit la cavité centrale dans toute la longueur, constituant ainsi la cellule du canal du col qui ne se segmente plus, contrairement à ce que nous avons vu chez les Mousses. Plus tard, le segment inférieur de la même cellule mère, segment devenu la cellule centrale, subit une nouvelle division en cellule embryonnale ou oosphère occupant le ventre de l'archégone et en cellule ventrale du col.

Les Marattiacées diffèrent quelque peu des autres Fougères par la formation tardive des archégones et par leur col court et peu proéminent; le ventre est plongé entièrement dans le tissu du prothalle, modifié de façon à l'entourer d'une assise de cellules tabulaires (M. Jonkman). Les Ophioglossées se rapprochent de la famille précédente; le col est plus court encore; la cellule basilaire semble manquer.

Les parois, d'abord très minces, des deux cellules du col, s'épaississcnt; le volume des cellules augmente, sans que leur protoplasma suive cet accroissement; leur noyau disparaît peu à peu. Les membranes se gélifient; imprégnées d'eau, elles se gonflent rapidement, se transforment en mucilage et, écartant brusquement les séries périphériques du col, elles s'échappent au dehors; le mucilage s'étale alors autour de l'orifice de l'archégone; quant au protoplasma de ces deux cellules, il se trouve ordinairement rejeté à ce moment. L'oosphère s'arrondit dans le ventre; son sormet montre une région plus claire, la tache réceptrice; son noyau demeure intact.

Les Equisétacées diffèrent peu des Fougères : les archégones se forment à la face supérieure du prothalle; la cellule basilaire inanque ; la cellule du col du canal, très atténuée supérieurement, ne traverse pas tout le canal; les quatre cellules supérieures du col s'allongent beaucoup et, s'écartant à leur sommet, se recourbent en dehors. Il se forme peu de mucilage et cette circonstance, jointe à la diøcie, expli- 
que la grande proportion des archégones non fécundés (M. Sadebeck).

Dans les Lycopodiacées, M. Fankhauser n'a pas rencontré de jeunes archégones; leur place était indiquée par le point d'insertion des plantules sur les prothalles. Elles paraissent occuper le fond des sillons sur la face supérieure. Ordinairement une prothalle ne porte qu'ure seule plantule; si celle-ci souffre ou meurt, un deuxième archégone peut se développer; il.est donc probable que plusieurs archégones se forment successivement sur le même prothalle, tant qu'il n'y a pas eu fécondation.

\section{HÉTÉROSPORÉES.}

GERMINATION DES MICROSPORES, PROTHALLE MALE ET ANTHÉROZOÏDES.

Ninus avons vu qu'en dehors de leur exospore les microspores étaient entourées d'une couche de plasma desséché provenant de la cavité diz sporange; c'est l'épispore qui ne constitue pas en réalité une membrane. Dans les Salviniacées, en effet, il réunit ensemble plusieurs spores. Nous avons vu qque, dans les $A z o l l a$, chaque sporange contenait ainsi plusieurs massulæ. Celles-ci deviennent libres et se fixent dans l'eau, par leurs glochides, aux objets qu'elles rencontrent, aux débris végétaux par exemple, mais surtout aux macrospores dont la région inférieure en est souvent toute couverte. La germination des microspores n'est pas connue.

Darrs les Salvinia il n'y a qu'une seule massula par microsporange, c'est-à-dire que l'épispore réunit toutes les microspores en une seule masse. La germination se fait à l'intérieur du microsporange; chaque microspore développe un filament (tube pollinique) qui traverse l'épispore, passe en les écartant entre les cellules de la paroi sporangienne et apparaît au dehors; mais auparavant le tube s'est déjà divisé par une cloison transversale en deux cellules: l'une inférieure, végétative, reste indivise et constitue le prothalle ; l'autre, supérieure, est l'anthéridie qui, par une nouvelle cloison transversale, se segmente en deux. D'après M. Arcangeli les choses se passeraient un peu différemment: 
le protoplasma du tube se différencie simultanément en trois cellules primordiales dont deux rapprochées au sommet; puis seulement apparaisssent les deux cloisons transversales. Quoiqu'il en soit, dans chaque cellule de l'anthéridie, le protoplasma se contracte, et se différencie en une petite portion plasmique qui reste sans emploi, et en une masse plasmique qui se divise en quatre cellules primordiales. Les cellules anthéridiennes se déchirent, les cellules primordiales devenues cellules-mères d'anthérozoïdes, s'échappent, et l'anthérozoïde que chacune contient se trouve mis en liberté; quant aux deux petites masses, elles restent dans l'anthéridie; ce sont les homologues de la cellule ventrale du canal dans l'archégone.

Ce prothalle unicellulaire semb!e très éloigné de celui des Fougères ; mais la différence paraîtra moindre, si l'on se rappelle les prothalles pauci ou même unicellulaires, portant des anthéridies (Fougères).

Les microspores des Marsiliacées (MM. Millardet, Arcangeli, Sadebeck) germent dans l'eau après s'être échappées de leurs microsporanges. A l'intérieur même de la spore, le contenu de celle-ci se divise encore en trois cellules : une inférieure, plus petite, reste stationnaire et représente le prothalle, les deux autres, plus grandes, se divisent chacune encore en quatre, comme dans les Salvinia; mais les huit cellules qui en résultent, au lieu de fournir directement autant d'anthérozoïdes, forment chacune une tétrade; il en résulte trente-deux cellules mères d'anthérozoïdes. Alors seulement la paroi de la spore est rompue et quelquefois les anthérozcïdes se montrent déjà en mouvement au moment de la déhiscence.

Le processus est le même chez les Pilularia et les Marsilia; les anthérozoïdes produits sont assez différents : dans les premières, ils possèdent deux cils et forment 4 à 5 tours de spire; dans les secondes, où il y a 12 à 15 tours de spire, les cils sont nombreux. Seuls les corps agiles des Marsilia entraînent avec eux une vésicule.

Dans les Selaginella (M. Millardet), les microspores, souvent très petites, possèdent une exospore à trois couches et une endospore formant une couche unique; leur contenu se partage d'abord en deux cellules. Il y a ainsi séparation d'une petite cellule végétatrice qui n’est jamais située à l'angile de déhiscence du tétraèdre, mais à un des 
trois autres angles; elle ne se développe plus, elle est munie d'un noyau, et c'est la seule cellule de la spore qui soit enveloppée d'une membrane de cellulose. M. Millardet fit voir qu'elle représente le prothalle, et son opinion a été ensuite adoptée universellement; cette découverte, étendue depuis aux Marsiliacées, est de la plus haute importance, car elle établit des liens certains entre les Isosporées et les Hétérosporées d'un côté, entre celles-ci et les Phanérogames (MM. Strasburger, Elfing) de l'autre. Le restant du contenu de la microspore se divise en 6 cellules (rarement plus jusqu'à 11, ou moins jusqu'à 4) dépourvues de membranes ou, autrement dit, primordiales. Dans le $S$. Kraussiana, deux seulement de ces cellules se multiplient pour former des cellules mères d'anthérozoïdes, et remplissent la microspore en résorbant leurs sœurs. Dans les $S$. Martensii et caulescens, elles se diviseraient toutes directement en cellules mères (M. Pfeffer). Celles-ci s'isolent, s'arrondissent, s'entourent d'une membrane; leur noyau disparaît. Pendant que les anthérozoïdes se forment dans leur intérieur, l'exospore se déchire suivant les trois arêtes convergentes; puis l'endospore se rompt. Les anthérozoïdes mis en liberté sont courts, renflés en arrière, et munis en avant de deux longis cils.

Les microspores des Isoetes (M. Millardet) sont le plus souvent des quartiers de sphère et possèdent un contenu finement granuleux avec noyau central et nombreuses gouttes huileuses. A la germination il se fait encore une petite cellule végétative ou prothallienne, munie d'une membrane de cellulose, et l'autre portion bien plus volumineuse du contenu de la spore constitue l'anthéridie; elle se divise en quatre cellules primordiales, dnnt deux ventrales résorbent les deux autres et donnent chacune deux cellules mères qui remplissent toute l'anthéridie. La rupture des parois de la spore met en liberté les anthérozoïdes, enroulés en hélice allongée et effilés aux deux extrémités qui portent chacune un pinceau de longs cils.

GERIINATION DES MACROSPORES, PROTHALLE FEMELLE ET ARCHÉGONES.

Le prothalle femelle, comme le màle, ne se nourrit jamais d'une façon indépendante, mais reste toujours une dépendance de la spore; 
ceci le différencie essentiellement du prothalle des Isosporées, dont il se rapproche plus par sa structure que le prothalle mâle, c'est-à-dire que la cellule végétative des microspores. Il subit aussi une réduction qui le rend comparable finalement au prothalle des Gymnospermes. Pour les archégones, l'analogie est plus grande aux deux limites.

Salviniacécs. - La macrospore des Salvinia (MM. Pringsheim, Prantl), qui possède trois enveloppes : endospore, exospore et épispore, germe normalement et forme de la chlorophylle même dans l'obscurité. Au sommet de la macrospore mûre, on remarque déjà un noyau; autour de lui, le protoplasma s'accumule en forme de ménisque contre la paroi supérieure. A la germination, ce ménisque augmente de volume; une cloison, insérée latéralement sur l'endospore, le sépare de la masse inférieure de la spore, qui se montre remplie de granulations protéiques. Il s'établit ainsi une séparation entre le protoplasma nutritif et le protoplasma évolutif de la spore; ce dernier est représenté par le ménisque, qui n'est pas autre chose que la cellule mère du prothalle. Une cloison verticale en sépare d'abord un tiers postérieur, dans lequel ne se formera ni archégones ni méristème. L'épispore se déchire alors en trois valves, entre lesquelles apparaissent les trois lobes du prothalle triangulaire dont le côté antérieur est opposé à l'angle stérile. Dans cette portion antérieure, une première cloison, presque horizontale, s'attache postérieurement à la paroi supérieure de la cellule fertile; puis, s'inclinant en avant, va s'insérer à la base de la face antérieure, qui s'est développée en hauteur; cette cloison divise la portion fertile du prothalle en une cellule antérosupérieure contenant le rebord antérieur, et en une cellule postéroinférieure confinant à l'angle stérile; cette portion fertile se constitue alors en méristème et devient active dans deux directions divergentes. La face postéro-supérieure du prothalle, inclinée d'avant en arrière et recourbée en selle, montre une crête longitudinale, sur laquelle apparaît bientôt le premier archégone, dirigé obliquement en arrière vers l'angle améristique stérile qui s'est développé en une protubérance. Le prothalle constitue alors un amas circulaire assez mıssif. Après le premier archégone, il s'en forme encore deux autres sur les flancs de 
la selle; ils sont situés sur une ligne transversale avec le premier. Si aucun de ces trois premiers n'est fécondé, de nouveaux archégones pourront encore naitre en direction acropète, c'est-à-dire plus rảpprochés du rebord antérieur méristique. Les deux angles antérieurs du prothalle se développent en deux prolongements ailés, qui persistent jusqu'à ce que la plantule se soit séparée de la macrospore. Il ne se forme point de rhizoïde.

Le développement de l'archégone est semblable à celui des Fougères, sauf l'absence de cellule basilaire : col à quatre séries dont les cloisons transversales sont ubliques, deux cellules du canal se transformant en mucilage: seulement ce mucilage soulève et arrache les quatre cellules operculaires ainsi que toutes les autres cellules du col non soudées avec le prothalle; les quatre cellules inférieures des séries, dites de fermeture, persistent seules.

Dans les Azulla les flotteurs augmentent de volume, arrachent et soulèvent la moitié supérieure lignifiée de l'indusie, qui se trouve plus tard rejetée sur le côté. Entre les trois flotteurs un canal étroit conduit jusqu'à l'exospore. Celle-ci se déchire en trois valves, et le prothalle d'abord, l'embryon ensuite, pénétrant dans le canal, écartent et repoussent de côté les trois masses du flotteur.

Le prothalle se développe a sommet de la macrospore comme ảans les Salvinia; quand il apparaît au dehors, il a la forme d'un disque obscurément triangulaire, un peu convexe, composé au milieu de plusieurs assises cellulaires, d'une seule à la périphérie (Azolla caroliniana); il est séparé inférieurement par une membrane mince et hyaline de la cavité de la spore qui est remplie de protoplasma. Un ar chégone apparait près du centre; il se compose d'une oosphère et d'un col à quatre séries, chacune de deux étages. S'il n'est pas fécondé 11 s'en forme encore un petit nombre d'autres autour du premier. A l'état adulte la partie proéminente du prothalle est presque hémisphérique, plate sur les bords qui s'allongent en trois ailes, dont une représente la protubérance stérile, améristique des Salvinia; le tissu est riche en chlorophylle. Pius tard le pied de l'embryon se trouve envaloppé par le prothalle comme par une gaine (M. Berggren). 
Marsiliacées. - Le sporocarpe des Pilularia étant imprégné d'eau, se fend au sommet en autant de valves qu'il possède de sores; il laisse échapper une goutte de mucilage qui entraîne macro et microsporanges; ceux-ci s'ouvrent; les spores, mises en liberté à leur tour, germent. Cette germination n'est pas encore bien connue ici; elle semble cependant très analogue à celle des Marsilia. Le protoplasme évolutif paraît se concentrer dans la papille et se séparer par une cloison de la masse plasmique, riche en amidon, qui remplit la cavité de la spore (plasma nutritif). D'après $M$. Arcangeli, le prothalle se développe dans cette papille, non par formation de cellules primordiales, mais par segmentation. Les recherches de cet auteur ne permettent plus, en effet, de considérer le tissu de la papille comme un archégone individualisé et le plasma nutritif comme un prothalle rudimentaire; quand l'archégone n'est pas fécondé, le tissu qui l'entoure se développe, en effet, comme un prothalle, acquiert de la chlorophylle, forme de nombreux rhizoïdes et tend ainsi à se nourrir d'une façon indépendante. Le col de l'archégone est ici fort allongé, ainsi que les cellules de fermeture placées à la base de ce col.

Dès que l'eau a pu pénétrer dans le sporocarpe de Marsilia à travers son enveloppe pierreuse, le cordon corné se gonfle, fait éclater la suture ventrale et se projette au dehors sous forme d'anneau, entraînart les sores; la partie dorsale du cordon ne tarde pas aussi à se gonfler et à s'allonger, les sores tendues sont arrachées de la partie ventrale de l'anneau où leur adhérence est moins forte; elles restent fixées par paires à la portion dorsale. L'indusie et la paroi du microsporange se détruisent rapidement; les macrospores sont mises en liberté. Comme dans les Pilularia, l'épispore, différencié en plusieurs couches, forme un volumineux bourrelet au sommet de la spore, mais en laissant libre un espace en forme d'entonnoir, au fond duquel la membrane propre de la spore proémine comme une papille arrondie et jaune-rougeâtre. Cette couleur est due au protoplasma évolutif finement granuleux de la papille; le reste de la cavité de la spore est surtout riche en amidon; il n'y a pas encore dans la macrospore mûre de membrane séparant ces deux masses; car, en comprimant très légèrement la spore, on 
voit les grains d'amidon pénétrer, sans résistance aucune, dans la papille (Hanstein).

Six heures environ après l'ensemencement une membrane se forme autour du protoplasma de la papille qui se différencie en une masse centrale et en une couche périphérique plus épaisse au sommet qu'à la base(Hanstein). Le protoplasma de celle-ci se divise en cellules primordiales de plus en plus petites qui forment une assise simple autour de la sphère centrale. Celle-ci s'entoure d'une membrane et constitue la cellule de la rangée centrale; les petites masses périphériques forment à leur tour leur paroi successivement du sommet à la base du prothalle; au-dessus de la cellule centrale quatre cellules se soulèvent et se divisent en deux étages par une cloison oblique. La cellule mère de la rangée centrale donne alors la cellule du col du caral, puis se divise en cellule ventrale du canal et oosphère. Le prothalle a aug.menté pendant ce temps de volume et a rompu l'exospore en trois valves, le mucilage, formé par les cellules du canal, écarte celles du col et vient s'étaler à la surface.

Selaginella. - La macrospore tétraédrique différencie plus que partout ailleurs son contenu avant la déhiscence, puisque, dans la spore fermée, les archégones existent déjà à l'état rudimentaire. La cavité de la spore est remplie d'une masse plasmique riche en graisse et en granulations protéiques irrégulières; au-dessus le prothalle, en forme de ménisque, se segmente d'abord en cellules primordiales qui s'entourent ensuite d'une membrane; à la germination il augmente de volume; six à sept semaines après l'ensemencement, il a ébauché quelques archégones; il rompt alors l'enveloppe de la spore. Peu avant 1a masse sous-jacente, par résolution de la majeure partie des granulations protéiques, est devenue trouble, puis s'est divisée en balles sphériques; celles-ci s'entourent de membranes successivement de haut en bas, et constituent ainsi la masse cellulaire qu'on a appelée endosperme et dont le développement n'est ordinairement achevé qu'après la fécondation. Pour la formation des archégones, une cellule superficielle, par une cloison transversale, se divise en une moitié supérieure ou cellule mère des séries du col, et une moitié inférieure 
ou cellule mère de la rangée centrale. Deux cloisons, perpendiculaires entre elles et à la surface, découpent la première en quatre qui formeront un col à deux étages. La deuxième pénètre encore par sa pointe entre les éléments du col; elle donne deux cellules du canal et l'oosphère.

Isoctes. - Quelques semaines après que la macrospore s'est échappée du macrosporange, le contenu de la spore se divise en cellules primordiales qui s'entourent ensuite de membranes. Ces cellules sont plus petites au sommet où elles semblent se multiplier plus activement. L'enveloppe de la spore se rompt à ce sommet en trois valves. L'archégone se forme comme dans les Selaginella; le col est à trois ou quatre étages, dont le supérieur seul est proéminent au-dessus du próthalle; la cellule ventrale du col existe très probablement, mais n'a pas encore été observée directement. Il n'y a, pas plus que dans le genre précédent, de cellule basilaire. Si le premier archégone n'est pas fécondé, il peut s'en former jusqu'à vingt ou trente autres d'après M. Kienitz-Gerloff.

Le prothalle femelle dans les Lycopodiacées hétérosporées est une formation endogène à un plus haut degré encore que dans les Rhizo-carpées; ce prothalle est aussi plus réduit. Dans l'un et l'autre cas s'établit, dans la macrospore, une différenciation en protoplasma évolutif et en protoplasma nutritif; dans les Rhizocarpées, ce dernier se trouve employé pour la construction du prothalle, puis, pour les premiers développements de l'embryon, sans même s'organiser en cellules. Dans les Sélaginellées, au contraire, il se divise en éléments cellulaires; dans le genre Selaginella, cette division est tardive. La partie inférieure végétative de la microspore est frappée d'un arrêt de développement temporaire seulement; et ce processus offre une certaine analogie avec les phénomènes du sac embryonnaire où le noyau secondaire reprend, après la fécondation seulement, il est vrai, son développement interrompu (M. Guignard).

Le prothalle indépendant des Isosporées offre ordinairement deux faces dissemblables dorsale et ventrale. D'après M. Leitgeb, il est tout d'abord vertical dans les Polypodiacées, et c'est la lumière qui 
déterminerait son orientation horizontale et la différenciation des deux faces; les rhizoïdes et les organes sexuels auraient un héliotropisme négatif; car en éclairant d'en bas des prothalles de Ceratopteris nageant sur l'eau, M. Leitgeb vit les rhizoïdes et les anthéridies se développer à la face supérieure non éclairée.

Quoi qu'il en soit, c'est à la face inferieure que les prothalles portent les organes reproducteurs dans les Polypodiacées (la plupart), à la face supérieure dans les Equisétacées, Ophioglossées et Gymnogramme. Dans l'un et J'autre cas la structure du corps végétatif est des plus simples; c'est un filament développé, tout d'abord en protonéma dans quelques Hyménophyllacées, mais donnant naissance desuite, dans les Polypodiacées et Equisétacées, à une expansion lamelleuse, sur laquelle ne se différencie qu'un coussinet formé de plusieurs assises de cellules; ce coussinet se change dans les Marattiacées en un corps cellulaire, lequel devient indépendant dans la Gymnogramme leptophylla, en perdant sa chlorophylle, et qui conduit ainsi au prothalle des Ophioglossées. Si l'on veut comparer cette génération sexuée, si peu différenciée, à celle des Muscinées, c'est dans les types inférieurs seulement de ce groupe que l'on trouvera des analogies; dans les types supérieurs la structure est notablement plus complexe. Le prothalle mâle des Hétérosporées est bien plus réduit, nous y reviendrons tout à l'heure. Leur prothalle femelle se rapproche de celui des Isosporées par son organisation, mais non par son mode de nutrition; caril a perdu toute indépendance et n'a d'autres ressources que la réserve alimentaire accumulée dans la spore. On dirait qu'une tendance se manifeste alors à économiser pour le jeune embryon cette nourriture et à ne plus la dépenser à l'édification du prothalle qui se réduit de plus en plus jusqu'à devenir tout à fait endogène. A cet état son développement ne sera plus nullement gêné, s'il reste fixé dans le sporange, adhérent lui-même au sporogone; et en réalité, c'est à peu près à cela que se réduit, scus le rapport de la génération femelle (du prothalle), la différence entre les Gymnospermes, le Ginkgo, par exemple, et les Selaginellées. L'endosperme des Archispermes qui ne se résorbe pas pour se reformer une seconde fois comme l'avait cru voir Hofmeister (M. Strasburger), est bien le repréRietsch. 
sentant du prothalle. Dans les Angiospermes, la réduction est poussée beaucoup plus loin; les synergides et les antipudes restent comme seuls représentants du prothalle, de l'archégone et de son col; par le développement, après fécondation, de leur noyau végétatif secondaire en endosperme, c'est encore avec les Selaginella qu'elles présentent le plus de rapports. Les archégones leur manquent totalement, quand, au contraire, ces organes montrent une manière d'être très uniforme depuis les Muscinées jusqu'aux Gymnospermes.

Dans ces deux groupes en effet, comme dans les Cryptogames vasculaires, nous trouvons toujours les mêmes parties essentielles, et seulement des différences secondaires. Le nombre des rangées et des étages du col est plus élevé dans les Muscinées; la cellule du col du canal s'y subdivise. Dans les Cryptogames vasculaires, elle reste indivise, le nombre des étages du col se réduit quelque peu, il peut n'être plus que de deux. Dans les Gymnospermes ce nombre varie entre un et trois, et les deux cellules du canal n'en font plus qu'une, le ventre ne se distingue plus du tissu du prothalle.

Les anthéridies des Isosporées sont notablement plus simples que celles des Muscinées; leur paroi se réduit à quelques cellules. Néanmoins, à la séparation des sexes, cette simplification fait encore un grand pas;l'enveloppedisparaît, l'anthéridie, réduite au tissu fertile,subit seulementles segmentations qui doivent conduire aux cellules mères. En même temps le prothalle n'est plus représenté que par une cellule (M. Millardet), et la génération mâle se trouve, comme la femelle, entièrement dépendante de la spore, avec une réserve nutritive bien moindre. Ici les analogies aveçles Phanérogames sont très marquées. Les grains de pollen des Gymnospermes (Hofmeister, M. Strasburger) comme ceux des Hétérosporées, séparent longtemps avant la déhiscence, par une cloison, la cellule végétative qui peut même se subdiviser; au sommet du tube pollinique, le noyau évolutif se partage en deux (ou même plusieurs) noyaux, autour desquels s'accumule le protoplasma; ce sont presque les divisions de l'anthéridie. Dans les Angiospermes (MM. Strasburger, Elfving) il se forme aussi deux cellules : l'une végétative peut encore se subdiviser en deux ou trois autres, mais ordinairement elle dure peu et son noyau seul persiste; 
l'autre forme le tube polinique, au sommet duquel émigre le noyau évolutif qui se segmente même encore en deux dans les Cypéracées. Ce qui différencie, sous ce rapport, les Phanérogames, c'est l'absence d'anthérozoïdes, c'est la fécondation par diffusion à travers une membrane.

Il semble qu'à la limite inférieure des Hétérosporées, il n'y a pas de terme de comparaison à chercher pour le prothalle mâle. Rappelons-nous cependant les prothalles pauci ou même unicellulaires des Fougères, portant des anthéridies. On avait cru voir d'abord dans ces faits une tendance à la diœcie ; on s'est aperçu ensuite que le manque d'espace et de lumière, que de mauvaises conditions de nutrition, étaient la cause réelle de ces apparitions anormales. Il résulte d'expériences faites par M. Prantl que les spores d'Osmunda, privées à la germination d'azote assimilable, ne donnent qu'un prothalle réduit, améristique; en lui fournissant de l'azote on peut le changer en prothalle à méristème. Dans le premier cas il ne se forme que des anthéridies; elles peuvent se former ou mэnquer dans le second; quant. auxarchégonesils sont toujours lies au méristème. M. Prantl penseque ladisparition des rhizoïdes, eu réduisantle prothalle aux substances nutritives de la spore, a pu déter'miner la formation de prothalles améristiques par les petites spores, méristiques par les grandes, et conduire ainsi à la séparation des sexes. Les expériences de M. Prantl et les faits cités plus haut montrent que les circonstances physiologiques ont pu avoir de l'influence sur la première apparition de prothalles uniquement mâles; mais elles n'expliquent pas encore l'absence des anthéridies sur les prothalles femelles. Cette absence n'est-elle qu'une conséquence indirecte de la coexistence de prothalles uniquement måles? ou bien une nourriture abondante en précipitant le développement du prothalle à méristème, peut-elle tendre à supprimer un des stades (les anthéridies) du développement lent ordinaire? C'est ce que l'expérience pourra vérifier jusqu'à un certain point. Mais quoi qu'il en soit, la corrélation certaine entre le développement r'udimentaire des prothalles et leur sexualité mâle, nous semble faire do l’apparition de pareils prothalles un phénomène capital dans l'histoire de l'évulution des plantes; nous reviendrons plus loinsur ce sujet. 


\section{EMBRYOGÉNIE}

Après avoir séparéla cellule ventrale du canal comme pour expulser de son sein les matériaux superflus, l'oosphère ne tarde pas à être prêt à la fécondation. Son noyau homogène redevient central en augmentant notablement de volume; son protoplasma granuleux s'éclaircit vers la région supérieure qui devient la tache réceptrice.

Les anthérozoïdes nageant dans l'eau viennent se fixer dans le mucilage qui surmonte le col de l'archégone; ils descendent dans ce $c o l$ en nombre plus ou moins grand: le premier anthérozolde pénètre dans l'oosphère avec laquelle il se confond; comme l'oospore qui résulte de la copulation s'entoure aussitôt d'une membrane, les anthérozoïdes suivants ne peuvent plus pénétrer.

A moins donc que plusieurs corps agiles arrivent au même moment sur la cellule femelle, la fécondation semble n'être produite que par un seul anthérozoïde. Dans cette fusion les parties homologues des deux éléments sexués paraissent se réunir : noyau avec noyau (corps de l'anthérozoïde), plasma avec plasma (cils de l'anthérozoïde); ce sont donc deux cellules différentes qui se confondent en une celiule unique.

Lafécondation par les anthérozoïdes n'est possible que dans l'eau ; comme les Muscinées, les Cryptogames vasculaires sont donc encore, à un moment donné, sous la dépendance de ce milieu qui n'est plus indispensable aux Phanérogames (en faisant abstraction bien entendu de leur vie végétative).

A près la fécondation, le col de l'archégone reste ouvert, tandis qu'il se ferme dans les Muscinées (M. Sadebeck). L'effet de la fécondation se fait sentir aussi sur l'archégone qui s'accroît tout d'abord avec le jeune embryon, de façon à ne pas cesser de l'envelopper. L'oosphère devenue oospore s'entoure aussitôt d'une membrane. Tout d'abord le noyau persiste; mais au bout de quelque temps, après 10 heures déjà dans les Marsilia, il se segmente et la première cloison cellulaire a: parait a la suite de cette segmentation; elle est suivie bientôt de 
deux autres, et l'embryon se trouve généralement découpé ainsi en huit octants; on peut, pour abréger, donner des noms à ces trois premières cloisons, et les appeler avec les auteurs allemands : basilaire, transversale et médiane (Hofmeister, Hanstein, MM. Berggren, Bruchmann, Duval-Jouve, Kienitz-Gerloff, Leitgeb, Pfeffer, Pringsheim, Sadebeck, Vouk).

La paroi basilaire est à peu près dirigée dans l'axe de l'archégone et perpendiculaire à l'axe du prothalle (Fougères); elle peut aussi être perpendiculaire au premier axe (Equisetum, Selaginella); elle sépare presque toujours la tige de la racine; son vrai caractère est qu'elle se forme la première. Les deux autres parois sont perpendiculaires à la basilaire et entre elles; la médiane, comme son nom l'indique, passe par l'axe du prothalle (quand il y en a un) et par celui de l'archégone; la position de la transversale se trouve alors déterminée par celle des deux autres; elle sépare presque toujours la tige du premier cotylédon.

Filicinées. -. Si l'un se restreint aux Fougères, les dénominations précédentes prennent un sens plus précis; comme le prothalle possède un axe manifeste, la position de la basilaire et de la médiane sont nettement indiquées, et par cela même celle de la trasversale. C'est tantôt et plus souvent celle-ci (Polypodium vulgare, Asplenizm Trichomanes, Pteris serrulata) qui apparait la deuxième, tantôt la médiane (Asplenium Sheperdi). Les Salvinia ont aussi un axe du prothalle; les $A$ zolla peuvent leul' être comparées. Enfin la macrospore de Marsilia étant couchée horizontalement, on peut considérer l'axe du prothalle comme vertical et dirigé en haut, ce qui à la vérité revient à dire qu'il n'y a point d'axe et que, dans cette position de la mascropore, c'est la pesanteur qui détermine l'orientation de l'embryon.

Ceci étant posé, on peut dire que dans les Filicinées quatre octants sont loujours situés en avant (par rapport au prothalle) de la cloison basilaire, quatre en arrière, puisque cette cloison est perpendiculaire à l'axe du prothalle; la transversale étant parallèle au même axe et rpendiculaire à celui du prothalle, séparera quatre octants situés 
an fond de l'archigno ou internes, de quatre autres tournés vers le col ou externes.

I.es deux octants antéro-externes forment toujours la première feuille ou le cotylédon. Des deux octants antéro-internes un seul donne le sommet de la tige, l'autre forme la deuxième feuille ou deuxième cotyledon. Les deux octants postéro-internes se changent en pied. Des ceux octants postéro-externes, l'un, diamétralement opposé à loctant cle la tige, donne la première racine, quand il y en a une; l'autre se trcuve comprimé et alrêté dans son développement par la premiere racine, ou bien il prend part à la formation du pied.

Lí premiere segmentation dans les octants se fait généralement par une cloison à pen près parallèle à la basilaire; mais recourbée de ficcon ì êtra plus éloirnnée de cette paroi à la périphérie de l'embiyon qu'à son centre; il se forme aussi dans la moiťié antérieure de l'embryon qui contient la tigg:, un disque ou article épibasilaire, composé de quatre cellules convergentes a centre; dans la moitié postérieure un article hypobasilaire. Chacune des cellules de ces deux disques se divise ensuite par une cioison parallèle à la surface de l'embryon ou péricline, en une cellule interne et une cellule externe, de sorte que chaque aricle se compose alors de quatre cellules axiles et de quatre periphriques; les premières sont l'origine du tissu libéro-ligneux axile, les sec'ondes celles du systeme cortical. Ces formations n'exis. tent pas toujours dans le pied.

Dans les Polypodiacés et Cyatéacées (Fig. 3), seules Fougères dont le développement snit connu, le prothalle est un peu incliné vers le sol ri'avant en arrière; l'archégone, dirigé en bas, a son axe à peu près perpendiculaire à celui du prothalle. La cloison basilaire s'écarterait in peu, d'après M. Sadebeck, de l'axe de l'archégone pour se rapprocher de l'horizon, avec lequel elle ne ferait plus qu'un angle de trente degrès (?).

La moitié épibasilaire de l'embryon est donc située en avant et en haut; le sommet de la tige est presque dirigé vers le zénith, la racine vel's le sol et en alrière, la première feuille en avant. Dans l'octant antéro-intérieur qui doit former le sommet de la tige, une première cloisnn, parallèle à la basilaire, mais concave ver's l'axe de l'octant, 
sépare une portion de l'article épibasilaire; une deuxième cloison, concave aussi vers le même axe, mais parallèle soit à la transversale, soit à la médiane apparaît ensuite; puis une troisième parallèle à la médiane ou à la transversale. Ainsi se decoupe dans cet octant une pyramide sphérique renversée triangulaire qui n'est pas autre chose que la cellule terminale à trois faces de la tige.

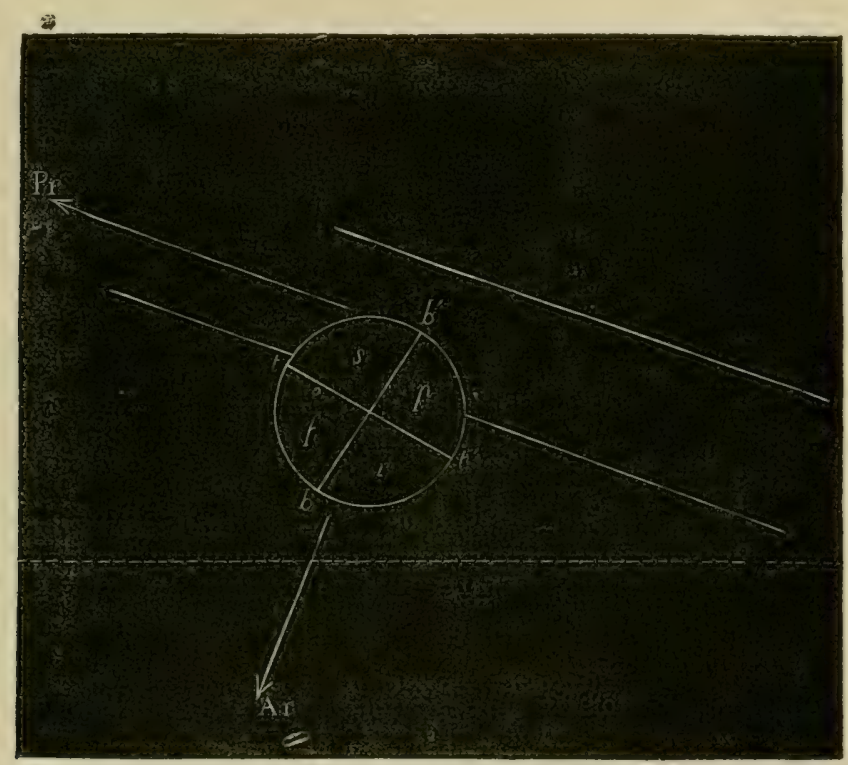

FIG. 3. - Fougères.

Les deux octants antéro-extérieurs, après avoir contribué à la formation de l'article épibasilaire, se segmencent par une cloison radiale à peu près parallèle à la médiane, puis par une péricline; des parois análogues à ces deux premières, continuant à apparaître dans les cellules résultantes, il se forme une surface à accroissement marginal d'où résulte la première feuille (ou contylédon), située à peu près dans

Ftg. 3. Schema de l'embryon des Fougères. - H-H, horizontale; $\mathrm{Pr}$, axe du prothalle; A $r$, axe de l'ar'chégone; $b b^{\prime}$, trace sur le plan du papier de la cloison basilaire; $t t^{\prime}$, de la transversale; la méciiane coïncide avec le plan du papier; $r$, racine; $p$, pie 1 ; $s$, sommet de la tige; $f$, première feuille. Mêmes lettres pour lè figurez suivantes. 
le plan du disque épibasilaire dont la partie adjacente prend probablement part à sa formation.

Les deux uctants postéru-intéricurs, se divisant par des cloisons perpendiculaires entre elles dans trois directions, donnent le pied, dont les cellules sont toujours plus grandes que dans les autres parties de l'embryon. Le pied ne cesse de s'accroitre que quand la deuxième racine commence à fonctionner (sauf chez les Hyrnénophyllacées qui n'ont pas de seconde racine).

L'octant diamétralement opposé au sommet de la tige, après avoir formé une partie de l'article hypobasilaire, acquiert encore deux cloisons parallèles à la transversale et à la médiane; la pyramide ainsi formée se découpe par une péricline en une cellule extérieure ou première calotte, et une cellule intérieure ou cellule mère du corps de la racine, la plus volumineuse de la jeune plantule. L'accroissement de la racine est très rapide; elle l'err porte souvent sur le cotylédon; elle détermine, avec celui-ci, la rupture de l'archégone.

La jeune plantule reste attachée nar le pied au prothalle dont elle tire, tout d'abord, sa nourriture; la racine et la feuilie apparaissent au dehor's et commencent à fonctionner; puis dautres racines et feuilles, bientôt de plus en plus grandes, prennent naissance sur la tigre dont le diamètre augmente dans les parties nouvelles, et ainsi se constitue successivement la Fougère adulte.

Dans les Marsilia (fig. 4) l'axe longitudinal de la spore et par suite

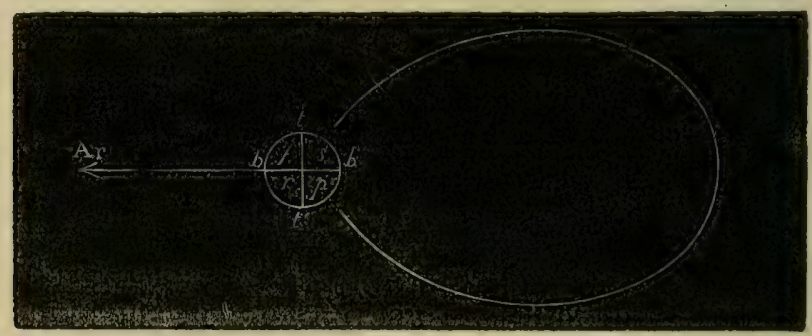

FIG, 4. - Marsilia.

celui de l'archégone sont horizontaux à la germination; on peut admettre que l'archégone des Fougères, déjà incliné vers l'horizon, a 
continué à s'en rapprocher, jusqu'à coïncider àvec lui. Toutes les parties de l'embryon conservent alors la même position dans l'archégone; l'axe du prothalle est vertical et dirigé en haut. Ce qui a été dit précédemment pour les Fougères, s'applique encore ici. L'octant antérointérieur, placé à côté de l'octant de la tige, se segmente cumme celui-ci tout d'abord; mais il s'accroit beaucoup plus vite et masque bientôt le sommet de la tige en se transformant en une ébauche de feuille; c'est la deuxième feuille, appelée aussi quelquefois deuxième cotylédon. Le premier cotylédon ne tarde pas à prendre la forme d'un cône, parce que son accroissement se fait suriout ici dans le plan de la cloison médiane; la portion correspondante de l'article épibasilaire contribue beaucoup à son développement. Le cotylédon finit par rompre l'archégone qui tout d'abord a grandi avec l'embryon.

Si l'on considère comme axe Iongitudinal du prothalle la crète de la selle(fig. 5), ce qui précéde pourra s'appliquer essentiellement aux

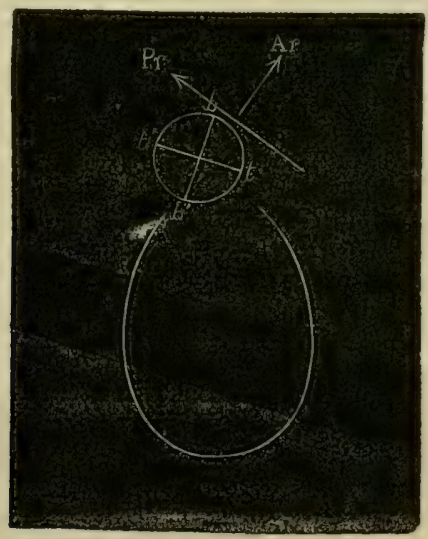

Fig. 5. - Salvinia.

Salvinia ; en effet la macrospore étant verticale, cette crête est inclinée de $45^{\circ}$ à peu près. M. Pringsheim figure la macrospore penchée de façon à rapprocher encore de la verticale l'axe du prothalle. Dans cette position qui en réalité importe peu, la première cloison est à peu près verticale. En tous cas l'hémisphère postérieur regarde vers la macrospore, plus que l'hémi sphère antérieur. Ici la racin 
manque el les quatre octants hypobasilaires sont employés pour le pied, après la séparation toutefois de l'article hypobasilaire. Les deux octants antéro-externes se développent rapidement en un demi-disque à la formation duquel prend part l'article épibasilaire; ce disque donnera plus tard l'écusson triangulaire ou premier cotylédon; son ébauche diffère de celle des Marsilia, où nous l'avons vue conique. Par suite de cet accroissement rapide, l'archégone est bientôt rompu; son col reste encore appliqué à la face dorsale du pied refoulé en arrière et en bas par l'écusson. Les deux octants antéro-internes se trouvent aussi, par le même écusson, dejjetés tout en bas. L'un de ces octants ne s'accroît que très peu, ses partitions ne tardent pas à devenir irrégulières, puis ses cellules périphériques se changent en trichomes; il s'épuise ainsi dans la formation de peils, comme dans les Ceratopteris parmi lıs Fougères. L'autre octant constitue tout d'abord une pyramide à trois faces comme dans les Marsilia; mais, après trois ou quatre divisions, celle-ci devient la cellule terminale à deux faces de la tige. Après la rupture de l'archégone l'article épibasilaire s'allonge beaucoup plus rapidement dans la portion qui correspond à cet octant que dans les autres; par suite le sommet de la tige, d'abord dirigé en bas, se relève peu à peu de façon à prendre une position différant presque de $180^{\circ}$ de la première.

Vuici comment les phénomènes ont été décrits dans l'Azolla par M. Berggren : la premıère division est perpendiculaire à l'axe longitudinal de l'embryon qui est un peu allongé; elle le découpe en deux cellules dont l'une, plus petite, sera provisoirement considérée comme supérieure. Deux cloisons successives, parallèles à l'axe longitudinal de l'embryon et perpendiculaires entre elles et à la première cloison (basiliaire), divisent l'embryon en huit octants, dont chacun est encore segmenté en deux par une paroi parallèle à la basilaire. L'embryon se compose donc de 16 cellules disposées en quatre étages. Les 4 cellules de l'étage supérieur forment le pied; des 4 cellules inférieures l'une donne le sommet de la tige, une autre un organe analogue aux premières feuilles, les deux dernières l'écusson. Le sommet de la tige se développe comme dans une plante adulte; il est d'abord droit, plus tard il se recourbe pour se diriger en haut. Vers le sommet du prothalle, les 
premières feuilles sont fortement concaves et non lobées. Quelques poils (des poils couvrent le sommet de la tige) se forment déjà en même temps que la première feuille. L'écusson forme d'abord un disque demi-circulaire autour du sommet, ses bords se rejoignent plus tard, de façon à constituer une gaine. Dans les deux articles épi et hypobasilaires des cloisons tangentielles divisent de bonne beure les cellules en externes et internes; celles-ci deviennent le rudiment du premier faisceau. Le prothalle est rompu près de l'archégone qui reste adhérent à la face dorsale du pied que le prothalle enveloppe comme une cupule. L'embryon se détache de la macrospore et l'écusson s'étale à la surface de l'eau. La moitié hypobasilaire (supérieure ici) semble produire une première racine dont le développement s'arrête bientôt ; elles est encore située entre le pied el le cotylédon, d'après les dessins de M. Berggren. Une deuxième racine apparait latéralement; elle est rıunie d'une coiffe; celle-ci ce désorganise plus tard et la racine se couvre alors de poils rhizoïdes disposés par verticilles.

M. Berggren n'est pas très certain de l'orientation de l'umbryon, par rapport à l'archégone; il pense, par analogie avec les Salvinia, que la moitié hypobasilaire est supérieure et dirigée vers le col obliquement. La fig. 1 (1) de $\mathbf{M}$. Berggren représente l'axe longitudinal de l'embryon perpendiculaire à celui de l'archégone, et l'auteur dit nettement que la première cloison est perpendiculaire ou à peu pres à cet axe longitudinal. Nous pensons donc que cette cloison ne s'écarte pas beaucoup de l'axe de l'archégone et, qu'en somme, il y a grande analogie dans l'orientation avec les Marsilia, seulementici les flotteurs maintiennent la macrospore dans une direction verticale. Pour le reste, ce développement ressemble beaucop à celui des Salvinia, et, dans toutes les Filicinées, l'embryogénie peut se ramener essentiellement̀ à un type uniforme, parr rapport aux deux axes du prothalle et de l'archégone, axes définis comme il a été dit plus haut. Il n'en est pas de même pour les Equisétacées, ni pour les Lycopodiacées hétérosporées; on ne sait encore rien de l'embryogénie des Lycopodiacées isosporées.

(1) Voy. Ann. Sc. Nat., 18s2, t. XIII, pl. 12, fig. 1, ou encore Revue des Sc. Nat , de Montpellier, 3º sér., t. I, 1881. 
Dans les Équisétacées (fig. 6), l'axe d'accroissement du prothalle et celui de l'archégone, sont à peu près verticaux; la cloison basilaire, perpendiculaire à peu près ì celui de l'archégone, fait un angle d'une vingtaine de degrés avec l'horizon. Les articles épi et hypobasilaires se développent moins régulièrement. La cellule terminale à trois faces de la tige se forme comme précédemmeut dans un des octants épibasilaires dont la première cloison, parallèle à la basilaire, sépare la portion correspondante de l'article; la tige prend, dès l'origine, un

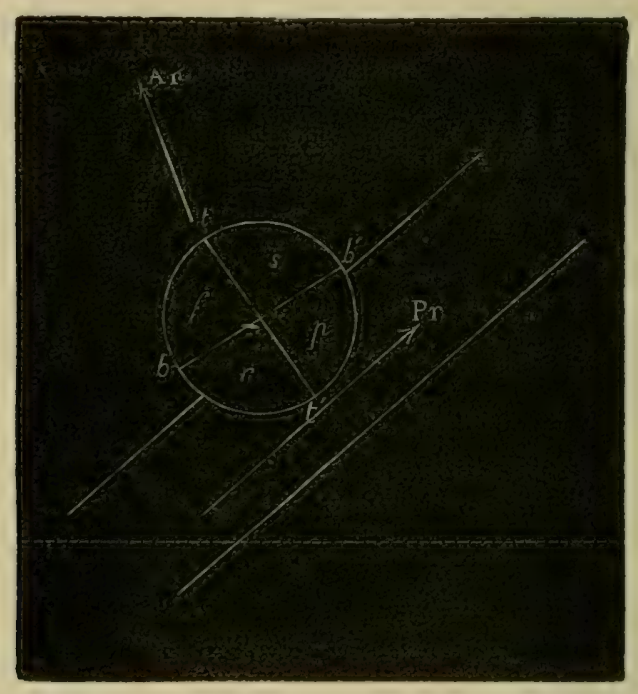

Fig. 6. - Equisetum.

accroisscment prépundérant et bientòt une position centrale. L'octant séparé du précédent par la cloison médiane, forme la deuxième feuille ou deuxième cotylédon; les deux autres octants épibasilaires, séparés des précédents par la transversale, se segmentent pour donner un cotylédon qui reste tout d'abord rudimentaire. Quand la cellule terminale de la tige a déjà fourni plusieurs segments, on voit s'ébaucher la première feuille issue de cette tige; elle s'accroît, ainsi que les deux cotyledons, par des partitions alternativement péri et anticlines, et ces trois formations, différentes d'origine, mais bientôt 
équivalentes dans leur développement, constituent le premier bourrelet annulaire, rudiment de la première gaine foliaire tridentée.

La moitié hypobasilaire de l'embryon montre une plus grande analogie avec la portion correspondante des Fougères. Deux octants, situés d'un mème côté de la transversale, donnent le pied toujours peu développé. Des deux autres octants, placés au-dessous du cotylédon, l'un, diamétralement opposé à celui de la tige, augmente beaucoup de volume en comprimant son congénère, forme un article hypobasilaire et se segmerite ensuite comme dans les Fougères pour fournir la cellule mère de la première racine; cette cellule est encore ici la plus grande de tout l'embryon (MM. Hofmeister, Duval-Jouve, Sadebeck).

Pour les Isoctes (fig. 7), les données ne sont pas très complètes,

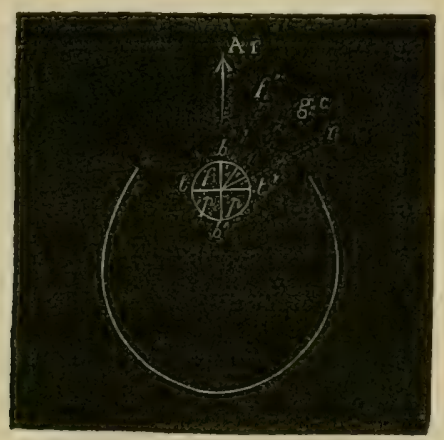

FIG, 7. - Isoütes.

malgré les récentes recherches de $\mathbb{M}$. Kienitz-Gerloff, dont je résume ici les résultats. Il se forme encore huit octants; la paroi basilaire qui sépare la tige de la racine, passe sensiblement par l'axe de l'archégone (vertical) et apparaît probablement la première; la transversale perpendiculaire à la direction du col, sépare, vers l'intérieur de l'archégone, la moitié inférieure du jeune embryon, tout entière employée à la formation du pied. Il n'y a pas d'articles basilaires; mais une première cloison parallèle à la transuersale divise chaque octant en une cellule attenante à cette transversale et une autre cellule écartée de la même paroi; il y alor's 16 cellules en 4 étages. Rarement 
cette cloison est remplacée par une péricline dans les octants postérosupérieurs (postéro-externes), dans lesqucls nait ici la racine. Le cloisonnement n'est pas régulier dans la moitié inférieure de l'embryon : le quadrant postéro-inférieur (postéro-interne) se développe peu; le quadrant antéro-inférieur s'allonge surtout dans le sens de la transversale. L'accroissement est semblable dans le quadrant antéro-supérieur qui forme le cotylédon, dans lequel on ne remarque point de cellule terminale. Le quadrant postéro-supérieur, enfin, donne naissance dans sa région inférieure (près de la transversale) à la première racine, dans sa région moyenne à la gaine cotylédonaire, dans sa région supérieure (près de la basilaire) à la première feuille; à la base de celle-ci apparaît le point végétatif de la tige. Dans un des octants antéro-supérieurs, à l'angle des cloisons basilaire et médiane, une cellule superficielle, située, par conséquent, au sommet de tout l'embryon, devient la cellule mère de la ligule du cotylédon. Cette cellule, par un développement qui rappelle les propagules de Marchantia, se transforme en une lamelle plate qui, s'incurvant par ses bords, embrasse comme une derni-gaine la base de la feuille. Le cotylédon s'accroît beaucoup plus vite que toutes les autres parties de l'embryon. La formation de la première racine n'est pas exogène, comme l'avait dit M. Bruchmann; cette racine ne possède point

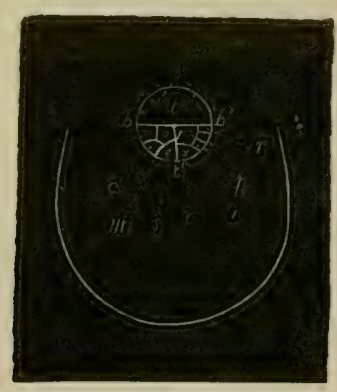

FIG. 8. - Selaginella.

de cellule mère unique, mais un méristème qui se cloisonne dans trois directions perpendiculaires et qui forme ainsi un tissu indifférent, dans lequel l'épiderme, la couche corticale et le cylindre central 
ne se différencient que plus tard; il n'y a point de cellule initiale unique pour le dernier. Le cutylédon ne présente pas de méristème particulier; un pareil tissu ne se distingue guère non plus dans le som-met très émoussé de la tige.

Ce développement diffère beaucoup ảe ce que nous avons vu dans les Filicinées ; celui des Selaginella (fig. 8) s'en écarte encore davantage, mais se rapproche de l'embryogénie des Phanérogames; la première cloison en effet est perpendiculaire à l'axe de l'archégone et la moitie externe (supérieure) de l'embryon se change en un suspenseur qui, en s'allongeant, fera pénétrer l'embryon dans l'endosperme, déjà organisé en cellules dans sa région supérieure au moment de la fécondation.

Le suspenseur s'allonge beaucoup, mais il ne se segmente ordinairement que dans sa région inférieure, adhérente à l'embryon, d'une façon irrégulière, par quelques cloisons longitudinales, obliques ou transversales. L'embryon est ainsi refoulé peu à peu, à travers la paroi de l'archégone et le prothaile, jusque dans l'endosperme; en même temps il continue à se diviser. Une deuxième cloison (transversale), perpendiculaire à la basilaire, sépare une cellule postérieure (origine du premier cotylédon, du pied et de la racine), d'une cellule antérieure (origine de la tige et du deuxième cotylédon). Dans cette dernière, une deuxième cloison un peu courbe, perpendiculaire à la transversale sur laquelle elle vient s'appuyer, et parallèle à la basillaire, découpe la cellule terminale de la tige tournée vers le fond de l'archégone et placee entre deux segments dont la base repose sur la basilaire. Chacun de ces deux segments est alors divisé par une cloison perpendiculaire à la fois à la basilaire et à la transversale, et passant, comme cette dernière par l'axe de l'archégona; ces deux cloisons respectent la cellule terminale; elles correspondent ensemble à la médiane. Chaque segment subit une nouvelle division parallèlement à la basilaire et se trouve ainsi découpé en une partie bicellulaire tournée en haut vers le suspenseur, et une partie bicellulaire tournée en bas vers le sommet de la tige; cette dernière partie forme un cotylédon par un développement analogue à celui des feuilles ordinaires. Les deux moitiés supérieures des segments constituent un disque; c'est 
l'article épibasilaire composé bientôt, par apparition de périclines, de quatre cellules périphériques et de quatre cellules internes; celles-ci donneront le faisceau libéro-ligneux axile. Dans le segment formé en dernier lieu, correspondant par conséquent au deuxième cotylédon, les cellules périphériques de l'article épibasilaire sont employées uniquement à la production du tissu cortical; dans le segment le plus ancien, elles forment en outre le pied, placé près du premier cotylédon, et la racine, placée entre le pied et le suspenseur. Le développement rapide du pied amène un déplacement de la tige; d'abord verticalement dirigée en bas, elle ne tarde pas à devenir horizontale; en même temps la racine dirige sensiblement en haut son extrémité libre, l'autre extrémité faisant suite à la tige. L'axe de l'embryon est devenu alors à peu près perpendiculaire au suspenseur. L'article épibasilaire a pris un développement considérable, entre les cotylédons d'un côté, le pied et le suspenseur de l'autre; les cotylédons aussi ont continué leurs segmentations; ıls paraissent être, presque dès l'origine, deux formations parfaitement équivalentes. Dans la portion de l'article épibasilaire qui doit former la racine, des cellules superficielles en. gendrent, par périclines, la première calotte; une des cellules de la couche hypodermique correspondante devient la cellule mère de la première racine; elle ne se distingue d'abord en rien de ses voisines, mais bientôt elle commence à montrer la segmentation caractéristique.

La cellule terminale à deux faces de la tige ne tarde pas à se changer en une cellule à quatre faces par l'apparition de deux nouvelles cloisons perpendiculaires aux deux premières; les segments se séparent alors par paires décussées; ensuite elle est coupée en deux par une cloison perpendiculaire aux deux cotylédons; l'une de ses moitiés continue à fonctionner comme cellule terminale, dans l'autre moitié se découpe une nouvelle cellule à quatre faces; ainsi s'opère la première dichotomie de la tige.

Les Selaginella s'écartent donc très notablement par leur embryo. génie, non seulement des Filicinées, mais encore de toutes les autres Cryptogames vasculaires. L'apparition du suspenseur les rapproche certainement des Phanérogames, quoiqu'il soit dèmontré aujourd'hui que cet organe n'est pas, dans ce vaste groupe, aussi général, ni par 
conséquent aussi important qu'on l'avait cru tout d'ahord. La direction de la première cloison, perpendiculaire à l'axe de l'archégone, est aussi différente de ce que nous avons vu chez les Isoctes et les $F i$ licinics; mais elle correspond à la première cloison de toutes les Phanérogames. Ce qui semble aussi important, c'est qu'il y a ici, pour l'origrine des deux premières feuilles ou cotylédons, une différence moindre que dans les autres Cryptogames vasculaires. Dans celles-ci la prernière feuille currespond presque toujours à deux octants, la seconde à un seul, quand clle n'est pas produite par la tige; dans les Selaginclla les deux premiers segments, presque équivalents, forment leurs cotylédons d'une façon identique

Des différences notables s'établissent entre les Cryptogames vasculaires, dès les premiers stades du développement, dans la position de leur's organes. M. Sadebeck avait cru pouvoir les expliquer par l'action de la pesanteur qui déterminerait la direction de la première cloison; celle-ci serait toujours à peu près horizontale et séparerait le protoplasma de l'œuf en deux moitiés, l'une positivement (racine), l'autre négativement géotropique (tige). Des expériences ont été faites sur des Marsilia quadrifolia par M. Leitgeb. L'axe longitudinal de la macrospore (et de l'archégone) est ordinairement horizontal à la germination dans la nature, la première cloison aussi est horizontale et c'est la moitié inférieure de l'embryon qui produit le pied; en fécon. dant artificiellement une macrospore dont l'axe était incliné sur l'horizon, cet axe devenait la ligne de plus grande pente de la première cloison; mais en plaçant la spore vertica!ement, la première cloison, verticale aussi, était dirigée dans n'importe quel sens. M. Sadebeck était arrivé à des résultats analogues en opérant sur des Marsilia clata et on employant la force centrifuge. L'influence de la pesanteur est donc très restreinte, ou même nulle dans certaines positions; les rapports de nutrition de l'embryon et de la macrospore peuvent l'emporter sur la pesanteur ; le pied doit toujours être dirigé en effet vers la macrospore. Telle est la conclusion de M. Sadebeck.

M, Leitgeb pense que, dans les Polypodiacées, la situation des difféRietsch. 
rentes parties de l'embryon est indépendante de la pesanteur, et déterminée uniquement par la position de l'embryon dans l'archégone et sur le prothalle.

Tous les cas observés jusqu'à présent, à ma connaissance, me semblent cependant susceptibles d'une seule et même explication.

Dans les Anthoceros (Hépatiques) on voit les cellules du pied s'allonger en longs filaments qui vont pénétrer dans le tissu du thalle, étendant ainsi le champ d'action du suçoir principal, c'est-à-dire du pied qui est chargé de nourrir l'embryon, même quand il est développé en sporogone. Ces filaments donnent une indication sur le mode d'apparition probable de la premiere racine; elle se trouve toujours, dans les Cryptogames vasculaires, indissolublement unie au pied; c'est un suçoir nouveau émanant du premier; c'est une portion du pied qui s'est adaptée spécialement de façon à aller fonctionner dans Je sol. Cela étant, le pied doit être dirigé forcément vers la réserve nutritive du prothalle ou de la macrospore, afin de transmettre les aliments au jeune embryon, que seul il nourrit dans le premier âge. Ces fonctions incombent ensuite à la racine qui doit auparavant se fixer dans le sol.

On peut admettre que tout d'abord la racine s'est dirigée dans n'importe quel sens; mais elle n'a pu nourrir'l'embryon, et celui-ci par conséquent n'a pu vivre que quand la racine, après avoir triomplé des obstacles qui lui barraient la route, a atteint le milieu propice, c'està-dire le sol; la direction utile s'est fixée ensuite par hérédité. Le géotropisme de la racine est incontestable; mais, à l'état embryonnaire, il ne vient qu'en seconde ligne; il faut en effet tout d'abord que la racine franchisse les milieux qui s'interposent entre elle et le sol. Ainsi donc :

$1^{\circ}$ Le pied sera tourné vers la réserve alimentaire, cela revient presciue à dire vers le fond de l'archégone, et la racine sera attenante au pied.

$2^{\circ}$ La racine sera placée et dirigée de façon à éviter les obstacles qui pourraient l'arrêter assez longtemps pour que l'embryon périsse dans l'intervalle.

$3^{\circ}$ Dans les limites tracées par les deux premières conditions, la racine sera géotropique. 
Dans les Équisćtacées, la couche du tissu prothallien, placée audessous du fond de l'archégone, semble, pour la racine, un obstacle facile à traverser; elle le traverse en effet en obéissant à son géotropisme. Le pied sera donc situé au fond de l'archégone, la racine à côté du pied, la tige diamétralement opposée à la racine; les cotylédons occuperont la place qui lcur sera ainsi laissée. M. Sadebeck dit que la raciṇe est inclinée de $30^{\circ}$ environ sur l'horizon et représente cette inclinaison à peu près comme dans notre figure schématique (fig. 6, page 188) ; cette position est en effet plus favorable au géotropisme de la racine, probablement même plus favorable au pied, vu la position réelle des archégones par rapport au prothalle et à ses lobes.

De même dans les. Polypodiacées, la racine occupera à peu près la région la plus inférieure de l'embryon; le pied sera au fond de l'archégone au-dessus de la racine; la tige et le cotylédon ont dès lors leurs places indiquées. Mais le pied a encore une orientation fixe par rapport au prothalle; cette position est déterminée par la loi précédente. Si le pied est toujours dirigé en arrière par rapport au prothalle, cela peut tenir : $1^{\circ}$ à ce que dans cette position, plus que dans toute autre, il se trouve situé au fond de l'archégone, à cause de l'inclinaison du prothalle; si on met par exemple le pied à la place de la première feuille, en laissant en position les octants $r$, il est évident (fig. 3, page 183) que les fonctions du pied se rempliront alor's plus difficilement; ou $2^{\circ}$ à ce qu'il se trouve ainsi tourné vers la source des aliments qu'il doit transmetíre à l'embryon, c'est-à-dire vers la région postérieure du prothalle couvert de rhizoïdes. Ces deux causes pourraient coexister. En tout cas, leır effet est le même, étant donnée Ja position habituelle du prothalle. En même ternps la racine occupe aussi son poste le plus favorable. On pourrait songer à apprécier approximativement la valeur relative de ces trois forces en changeant l'inclinaison du prothalle, d'arrière en avant par exemple, ou de gauche à droite. Remarquons cependant que cette position du prothalle est tout à fait générale dans les Polypodiacées, qu'elle doit avoir des causes particulières, qu'elle est donc très probablement ancienne. Dès lors le pied et la racine ayant agi d'accórd depuis longtemps pour donner toujour's la même place à la basilaire, la position 
de cclle-ci a bien pu acquérir une certaine fixité heréditaire, et en changeant artificiellement la direction d'un prothalle, on ne réussira peut-être pas pour cela à modifier l'orientation de l'embryon. M. Leitgeb a très ingénieusement obtenu des prothalles de Ceratopteris portant leurs archégones à la face supérieure; l'embryon avait toujours la même orientation conforme aux exigences du pied, mais contraire cette fois à celles de la racine; les premières sont essentielles; le géotropisme de lá racine ne peut, je l'ai dit, être considéré dans l'embryon que comme une force secondaire; il semble donc très admissible que l'hérédité ait pu en venir à bout.

Dans toutes les Hétérosporées, la macrospore possède une enveloppe résistante qui serait pour la racine, engagée dans la cavité de la spore, un obstacle tel que l'embryon périrait fatalement; la racine ne pourra donc pas se diriger vers le fond de l'archégone; mais elle se rapprochera du col et s'orientera, en tous cas, de façon à ne pas pénétrer dans l'intérieur de la macrospore; elle aura une force sporifuge quil'emportera nécessairement sur le géotropisme; le pied, par: contre, est sporipète. On pourrait m’objecter ici la propriété de la racine de contourner les obstacles qu'elle rencontre; il faut cependant reconnaître que la racine enfoncée dans la spore jetterait la perturbation dans la réserve alimentaire et qu'il lui faudrait un temps considérable pour en ressortir. L'embryon périrait forcément dans l'intervalle.

Nous avons dit que dans les Marsilia (fig. 9) l'axe de la spore est ordinairement horizontal; la racine tend donc à prendre la position $r^{\prime}$ or", le pied la position $p^{\prime}$ op $p^{\prime \prime}$. Mais ces deux organes sont enchaînés l'un à l'autre; l'hémisphère racine-pied aura donc une position intermédiaire. si les deux forces sont égales, et on peut sans doute approximativement les considérer comme telles, puisqu'elles sont aussi essentielles l'une que l'autre, la basilaire devra passer par l'axe de l'archégone; elle n'aura pas d'autre condition à remplir vis-à-vis de ces deux forces; mais le géotropisme intervient alors et elle devient horizontale. Dans toute autre inclinaison de la spore, l'axe de l'archégone sera, pour le même motif, la ligne de plus grande pente de la basilaire; dans les deux positions verticales le géotropisme se trou- 
vera annulé et la basilaire pourra tourner autour de son axe vertical. C'est l'explication complète des expériences de M. Leitgeb. D'après M. Sadebeck, la basilaire ne coïnciderait jamais tout à fait exactement avec l'axe de l'archégone, sans indiquer la nature de la déviation qui doit être bien légère, puisqu'elle a échappé à M. Leitgeb. Si elle existe d'une façon appréciable et dans un sens déterminé, ce n'est sans doute pas au géotropisme, trop faible dans l'embryon pour réagircontre la force sporifuge de la racine, qu'il faudrait l'attribuer, mais bien à une certaine inégalité des deux forces sporifuge de la racine et sporipète du pied; la nutrition par la réserve de la spore précédant la nutrition par la racine, le pied pourrait l'emporter quelque peu.

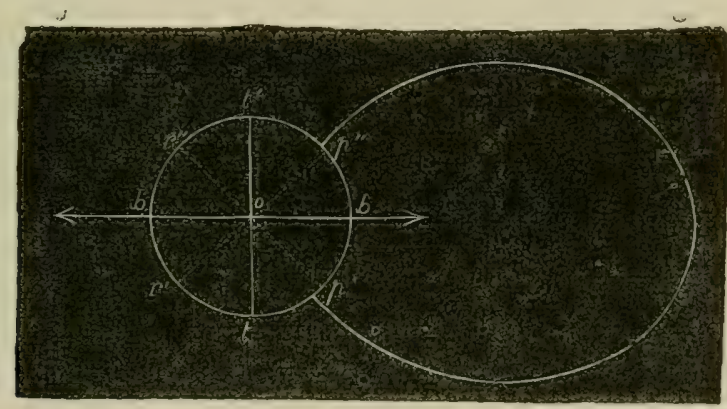

FiG. 9. - Marsilia.

On pourrait invoquer ici, en faveur de l'horizontalité de la basilaire, l'hérédité que j'ai mise en avänt tout à l'heure dans les Fougères (pour les positions inclinées de la spore de Marsilia). Mais la position horizontale de la macrospore ne peut être considérée comme aussi fixe que celle du prothalle, chaque objet flottant dans l'eau, le moinüre vent derant nécessajrement la modifier à chaque instant; puis l'hérédité s'attaquerait surtout ici à des conditions sine qua non, point accommodantes comme le géotropisme; elle ne saurait donc être invoquée.

Les dessins de M. Arcangeli pour les Pilularia , ceux de M. Beriggren pour les $A \approx$ olla, me semblent conciliables avec l'interpretation précédente; une explication est cependant nécessaire pour le dernier 
cas. 2I. Derggren dit n'avoir pas pu déterminer la position de l'emblyon par rapport à l'archégone; il suppose que l'hémisphère racinepied est situé obliquement vers le col; mais d'un autre côté il dit expressément que la basilaire est perpendiculaire à l'axe longitudinal de l'embryon, et sa figure 1 (voy. Ann. sc. nat., 1882, ou Revue de Montpellier, 1881) représente cet axe horizontal et perpendiculaire au col; il est donc permis d'admettre que la basilaire passe à peu prè.s par l'axe de l'archégone. Comme les macrospores d'Azolla flottent sur l'eau, avec leur appareil natatoire, ce dernier axe est à peu près vertical.

A en juger par les dessins de Hofmeister (Beiträge) et ceux de M. Fankhauser (Bot. Zeit., 1872), l'interprétation est encore valable pour les Botrychium et les Lycopodium; dans les deux plantes les prothalles sont massifs et il faut admettre une force prothallifuge, au lieu de sporifuge; cela revient ài même.

Les Salvinia sont dépourvues de racine. Il semble donc que le pied, restant seul en jeu, devrait se placer dans la position $p^{\prime}$ op $p^{\prime \prime}$ de la figure 9 par rapport ì la macrospore, ou occuper tout le fond de l'archégone. Il n'en est rien. La basilaire se trouve approximativement, par rapport à la spor'e et aux deux axes de l'archégone et du prothalle, dans la position $b b^{\prime}$ (fig. 5, page 185). D'après M. Pringsheim, l'hémisphère $b t b^{\prime}$ forme tout entier le pied. D'après cela on pourrait admettre, en tenant compte surtout de la première racine rudimentaire des Azolla si voisines, que cette absence de racine n'est point primitive; qu'une racine, autrefois existante, était dirigée non seulement de façon à éviter la spore, mais aussi vers le point de moindre résistance du prothalle assez développé, c'est-à-dire presque exactement vers le col de l'arrchégone ; cette position, jointe à la force sporipète du pied, orientait tout l'embryon, même par rapport au prothalle, tel qu'il l'est récllement. La moindre ébauche de racine, existant encore, eût suffi pour faire sentir son influence, puisqu'il ne s'agit que des premières segmentations et les Salvinia rentraient encore, sans grand effort, dans la loi commune. Mais les recherches de $\mathbf{M}$. Leitgeb ne s'accordent pas avec celles c.e M. Pringsheim. Le naturaliste autrichien décrit le pied comme formé par la portion inférieure de l'hé- 
misphère $b t^{\prime} b^{\prime}$ qui produit la tige et le cotylédon; l'autre moitié de l'embryon se réduirait ì un renflement ì la base du pied qui correspondrait à celui des mousses. Il faudrait supposer alors que la régression de la racine a fini par atteindretoute la moitié correspondante de l'embryon, qu'un nouveau pied a dù se développer' pour sup pléer à l'ancien, mais que l'orientation de l'embryon n'en a pas moins été fixée par cette première ébauche de l'hémisphère pied-racine. Ce serait sans doute aller un peu loin dans la voie des hypothèses. Des réserves doivent être faites pour les Salvinia.

Les Rhizocarpées à racine r'en montrent pas moins deur tendances opposées du pied et de la racine, qui arrivent cependant encore à se mettre d'accord ; mais à mesure que le prothalle s'itale moins en dehors de la spore, cet accord deviendra plus difficile. Dans le prothalle cndogène la racine devra se diriger de dedans en dehors vers l'orifice de la spore, son orientation sera donc plus étroite que dans les Rhizo. carpées; elle sera donc mieux déterminée, quand la macrospore restera fixée dans son sporange et celui-ci sur le sporogone, c'est-à-diı e sur la plante; des téguments séminaux viendront en effetalors la protéger, ne laissant libre qu'un étroit orifice, le micropyle, vers lequel la racine devra rigoureusement se diriger. D'un autre côté, la réserve nutritive de la spore, au lieu de former une masse unique, facile à absorber par la large surface du pied qui la touchait presque directement, cette réserve s'organise en cellules. Il est même possible, sans que je m'en explique le motif, que cette modification soit corrélative de la réduction du prothalle. En tout cas, le pied sera obligé de s'enfoncer d'avantage dans la spore pour aller y chercher les aliments renfermés dans les cellules de l'endosperme. De là un antagonisme réel entre le pied et la racine; cet antagonisme a dû devenır le point de départ d'une série de transformations qui ont abouti finalement aux résultats suivants $: 1^{\circ}$ la racine est dirigée constamment, dans le prothalle endogène, vers l'orifice de la spore, ou vers le micropyle, son successeur physiologique à notre point de vue actuel; $2^{0}$ le pied a disparu, soit en se changeant totalement en racine, soit par suppression ; on conçoit cependant qu'il ait pu aussi s'adapter à ces nouvelles conditions, ct le scutelium des graminées n'est peut-être qu'un pied 
ainsi modifié ; son rôle physiologique n'infirmerait pas cette interprétation : $3^{\circ}$ comme conséquence de ce qui précède, le rôle du pied a été transporté à l'embryon lui-même et surtout aux cotylédons qui absorberont désormais la réserve alimentaire ; $4^{\circ}$ un organe nouveau, auxiliaire, s'est développé; c'est le suspenseur qui refoulera l'embryon vers cette réserve et pourra même contribuer quelquefois à sa nutrition dans le premier âge (1). Ainsi il aura été obvié aux inconvénients pouvant résulter de l'antagonisme signalé plus haut.

Mais ces modifications embryogéniques, corrélatives de beaucoup d'antres, ne se sont faites que très lentement, et de la route, très longue assurément, qui a dû conduire autrefois des cryptogames aux phanérogames, il ne reste que quelques tronçons épars; ils peuvent cependant fournir des indications précieuses.

Les Isoetes et les Selaginella représentent de pareils tronçons. Leur embryogénie s'écarte notablement de celle des Rhizocarpées. Les premie:rs organes de l'embryon n'occupent plus les mêmes positions relalives. Les Isoctes montrent encore des octants; mais on peut voir déjà que cette formation est loin d'avoir l'importance qu'on a cherché à lui attribuer, qu'elle ne constitue, en somme, qu'un moyen, et c'est le but surtout que nous devons envisager. Nous pouvons encore vérifier ici les lois précédentes.

Dans les Isoetes quatre nctants ( $p, p$, fig. 7 , page 189), employés à la formation du pied, s'interposent entre le cotylédon $f$ et la racine $r$. Le pied et la racine se touchent toujours, séparés seulement par la transversale; mais leurs rapports avec les octants ne sont plus du tout les mêmes. La racine est d'abord presque horizontale, néanmoins elle est déjà orientée vers la large ouverture de la macrospore. Le développement particulier du pied, décrit par M. Kienitz-Gerloff, doit avoir furcément pour effet de la diriger encore plus en haut et de l'écarter ainsi des parois de la spore; en effet, la partie antérieure du pied, placée sous le cotylédon, se développe davantage et s'alionge surtout transversalement; elle refoule donc en arrière et en haut la partie postérieure (à droite de la figure) qui réagit à son tour sur la racine dans le senś indiqué plus haut. C'est donc la largeur plus grande du

(1) Voy, M. Guignard, Recherches d'embryogénie végétale (Ann. sc. nat., 1881). 
pied et son dévcloppement particulier, qui lui permettent encore ici de coexister avec la racine. Enfin je dois insisier sur la large déhiscence de la spore, relativement aux Rhizocarpées; elle donne bien plus de latitude à la racine.

Les Selaginella (fig. 8, page 190) ne forment même plus d'octants. Le suspenseur des phanérogames ou proembryon apparait ici pour la première fois ; la basilaire, perpendiculaire cette fois à l'axe de l'archégone, le sépare de l'embryon proprement dit, qui seul est traversé par la transversale $t t^{\prime}$. Vient ensuite la cloison III qui découpe le sommet de la tiges (M. Pfeffer). La première feuille se forme en $c$, le pied en $p$, la racine en $r$; la deuxième feuille apparaît en $c^{\prime}$ et est, au bout de peu de temps, équivalente à la première, c'est-à-dire que les deux cotylédons sont déjà presque égaux. La différence essentielle avec les Isoctes, c'est l'interposition du suspenseur entre la racine et la première feuille à la place occcupée dans ce dernier genre par la gaine cotylédonaire $(y, c$, fig. 7 , page 189). Le pied occupe une place bien moindre dans l'œuf. Le suspenseur tout d'abord se développe rapidement et repousse encore plus loin dans la macrospore la racine déjà mal orientée; en mêmetemps, il plonge tout l'embryon dans l'endosperme; on dirait que la fonction d'absorber celui-ci commence déjà à être exercée par l'embryon, dont les premières exigences l'emportent d'abord, en tout cas, sur les tendances de la racine. Le pied ne se développe qu'après l'allongement du suspenseur, et c'est l'accroissement même du pied qui donne à la racine l'orientation nécessaire, car non seulement il rend horizontale la tige d'abord dirigée en bas, mais encore il refoule nettement en haut la racine (voir les dessins de M. Pfeffer, Bot. Abhandl, von Hanstein, 1871), à laquelle la très large ouverture de la macrospore est éminemment favorable dans ces conditions. Un rétrécissement notable de l'orifice de communicatiun de la spore avec le monde extérieur, doit évidemment amener de notables changements dans, les dispúsitions précédentes.

Nous sommes encore loin des phanérogomes; mais il est difficile de ne pas considérer les Selaginclla comme une étape intermédiaire entre ceux-ci et les cryptogrames, et de ne pas attribuer une grande influence, sur les changements ultérieurs, au développement des tégu- 
ments séminawx qui viennent protéger la macrosporange, et qui le protègent d'autant mieux que le micropyle est plus étroit.

Au point de vue embryogénique, les Isoetes et les Selaginclla représentent en quelque sorte des traités de paix boiteux dans la lutte entre le pied et la racine; l'antagonisme de ces deux organes n'a pas dû contribuer pour peu à la disparition ultérieure des stades de transition entre les cryptogames vasculaires et les phanérogames.

Les considérations précédentes auront mis en évidence, je l'espìre, l'existence, dans l'embryogénie végétale, d'un facteur négligé jusqu'ici, mais nullement mystérieux, et résultant simplement des nécessités de l'exis tence. : la force sporifuge de la racine.

Elles contribueront à donner de l'embryogénie de certains groupes une explication plus rationnelle que celle de tendances innées ou d'orientation fixe par rapport à tel ou tel axe, et aideront à ramener à des causes purement physiologiques certains phénomènes évolutifs.

Au point de vue embryogénique les Cryptogames vasculaires présentent aussi jusqu'à un certain point des analogies avec quelques Hépatiques. Dans ce dernier groupe les premières divisions de l'œuf sont assez variables. Chez les Riccia il se forme tout d'abord huit octants, mais ils se développent tous de la même manière. Dans les Marchantia la première cloison est perpendiculaire à l'axe de l'archégone, puis il se forme huit cellules disposées en deux étages; les quatre octants inférieurs donnent le pédicelle, les quatres supérieurs la capsule; mais ce pédicelle est-il bien homologue du pied des Fougères? Darıs les Jungermannices la première cloison est encore perpendiculaire à l'axe et sépare inférieurement une cellule qui ne se divise que peu ou point, et qui forme un appendice à la base du pédicelle, produit, ainsi que la capsule, par la cellule supérieure. Dans les Anthocerus, dépourvus de pédicelle, l'embryon présente deux ou trois étages de quatre cellules; l'étage inférieur forme un v́rai pied, le reste la capsule.

Les deux derniers cas correspondent mieux aux Cryptogames vasculaires; la première cloison sépare le ou les suçoirs de tout le reste du sporogone. Dans les Anthocerns les deux parties de l'embryon sont divisées en quatre, comme dans les Fougères par exemple, ce qui, joint aux filaments rhizoïdes, doit les faire considérer comme plus rappro- 
chés des Cryptogames vasculaires que toutes les autres Hépatiques. Mais s'il y a là des indications de parenté, la limite inférieure des Vasculaires n'en est pas moins très tranchée.

\section{APOGAMIE.}

L'acte sexuel n'est pas toujours indispensable pour la formation du sporogone. M. Fanlow a montré le premier que lesprothalles de Pteris aquilina peuvent produive, sans fécondation, des bourgeons feuillés, d'où résultent des plantes ordinaires. M. Da Bary s'est occupé ensuite du même sujet, et a montré que les jeunes plantules de Pteris cretica, Aspidium filix mas var. cristatum (var. des jardins), et Aspidium falcatum, prennent toujours naissance sul le prothalle par bourgeonne. ment agame. Des faits analogues ont été encore observés par M. Sadebeck sur Ie Todea africana, par Mif. Mer et Goebel sur les Isoetes.

Quand, dansle Pteriscritica par exemple, le prothalle à méristìme et déjà cordiforme, semble sur le point de produire des archégones, un groupe de quelques cellules, trois à quatæe oldinairement, placéss à la face inférieure du coussinet et un peu en alrière du sommet, forment une protubérance pointue qui devient une feuille par un développement analogue, non à celui des cotylédons, mais à celui des feuilles ordinaires d'une jeune plantule. Elle a sa pointe recourbée en dedans, sa face supérieure tournée vers le prothalle; elle occupe donc la même position que la première feuille de l'embryon. De bonne heure elle se couvre de poils unisériés, ủont l'extremité inférieure se transforme ordinairement plus tard en poils écailleux. A la base de la feuille prend naissance le sommet de la tige, recouvertbientôt de poils et d'écailles, et au-dessous de ce sommet s'ébauche ensuite la deuxième feuille, écartáe de $1 / 3$ ou $3 / 8$ de circonférence de la première. Le développement se poursuit comme dans une plantule normale. La première feuille n'est encore que peu différenciée, sa nelvure médiane est marquée par un mince faisceau qui se ramifie dans le limbe, et qui s'arrète brusquement daus le prothalle ou șétend jusque vers le milieu du coussinet; là il se compose de vaisseaux courts et fusiformes dont 
La formation est très précoce. La première racine prend naissance, par voie endogène, à la base du pétiole et à la face externe du faisceau, ou mème dans le prothalle, un peu au-dessous de l'insèrtion du pétiole. Au bout de deux ou trois ans la fougère, ainsi constituée, porte des spores. L'apparition du bourgeon apogame, de même que la fécondation, arrête le développement du prothalle; si le bourgeon avorte de bonnə heure, le prothalle peut reprendre son développement, mais il a lieu alors d'une façon anormale; le prothalle se flétrit ensuite, après avoir produit fréquemment des ramifications adventives, comme les prothalles ordinaires. Les ramifications, devenues indépendantes, se transforment en prothalles secondaires et produisent des anthéridies, mais point d'archégones; enfin elles donnent fréquemment des bourgeons agames feuillés comme le prothalle dont elles dérivent.

Quelquefois les archégones sont déjà plus ou moins formés dans les plantes précitées, au moment de l'apparition du bourgeon; ils semblent toujours avorter, mais à un état de développement très différent; il y a donc une série de degrés intermédiaires depuis l'absence des archégones jusqu'aux archégones entièrement formés.

Dans les Isoctes lacustris et echinospera les phénomènes diffèrent assez notablement de ceux des Fougères. Tout d'abord les macrospores qui donnent lieu à des cas d'apogamie, restent en place dans leur sporange et sur leur feuille; ces macrospores peuvent même ne pas se produire. Ensuite tout le pied d'Isoctes se développe irrégulièrement; on en trouve, dans ces cas, qui ne possèdent ni macrospores, ni microspores, mais une jeune plantule d'Isoetes implantée à la place ä'un sporange. Le prerrier rudiment de cette plantule qui ne diffère pas de celui d'un sporange, s'ébaliche déjà, quand la feuille mère est encore en voie de developpement; le faisceau de celle-ci lui envoie une branche; le bourgeon ne tarde pas à produire feuilles et racines; il est mis en liberté par destruction successive de la plante mère. On trouve des touffes entières de plantes ainsi produites, et elles se régénèrent à leur tour de la même façon; il semble donc y avoir là des dispositions héréditaires. D'après M. Mer, c'est le mode de nutrition qui détermine ces phénornènes; quand le sol est pauvre ou quand les plantes croissent serrées, la reproduction sexuée fait place à une multiplication 
par bulbilles qui peuvent se détacher de bonne heure de la plante mère, mais qui dans les individus vigoureux se développent sur celleci en une plante nouvelle. Un même individu peut présenter des macrosporanges sur certaines feuilles, des bulbilles sur d'autres; enfin M. Mer a observé les deux formations sur une seule et même feuille. Le mode de nutrition exerce aussi de l'influence sur les organes reproducteurs normaux; dans les individus vigoureux en effet $M$. Mer a vu augmenter la proportion des macrosporanges par rapport aux microsporanges, jusqu'à suppression de ces derniers.

Mais, quoi qu'il en soit, la formation, même exceptionnelle, d'une plantule nouvelle aux dépens d'une macrospore, non détachée de son macrosporange, mais restée en place sur la plante mère, c'est-ả-dire la provenance directe d'un sporogone du sporogone précédent, n'en constitue pas moins un fait des plus importants, si on le rapproche de ce qui se passe normalement dans les Phanérogames.

M. de Bary a àonné le nom d'apogamie à ce mode de production de la plante feuillée. L'apogamie semble apparaître d'une façon brusque et coïncider, quelquefois au moins, avec l'apparition d'une variété; ce bourgeonnement agame donne ordinairement des plantes normales, quelquefois cependant aussi des plantes anormales par leur position, leur orientation et leurs premières ramifications.

\section{MULTIPLICATION VÉGÉTATIVE.}

Nous avons déjà vu que les prothalles indépendants peuvent se multiplier par bourgeons adventifs ou ramifications latérales. Dans les Hyménohyllacées le protonéma rameux produit au-dessous des cloisons antérieures de ses cellules, des filaments latéraux qui se changent fréquemment en prothalles lamelliformes, bientôt indépendants. Le prothalle des Trichomanes possède sans doute aussi des organes de reproduction végétative dans ses cellules marginales en forme de bouteille; dans les Osmunda de pareilles cellules marginales s'aliongent en filaments, deviennent indépendantes et se transforment en nouveaux prothalles. Nous avons signalé aussi une propagation analogue dans les Gymnogramme et les Equisetum, etc. 
On la reirouve encore sur la génération sexuéc. Tris fréquemment des buurgeons ou pousses adventives se forment chez les Fougères à la base du pétiole, sur la nervure principale de la feuille, à l'aisselle ou sur la nervure principale des folioles, à la pointe même de la feuille. C'est une cellule superficielle unique ou un groupe de semblablables cellules qui constitue le premier rudiment de ces propagules. Dans certains cas ils produisent de longs stolons souterrains (Struthiopteris germanica) qui, pourvus d'écailles, redressent leur sommet et viennent épanouir au-dessus du sol une couronne de feuilles; ces stolons peuvent même se renfler en tubercule à leur sommet (Nephrolepis undulata). Les longues feuilles pendantes des Chrysodium, Woodwardia, etc., viennent toucher le sol par leur pointe, s'enracinent et y développent de nouvelles pousses. Dans les Ophioglossum, c'est sur les racines de la plante que se produisent ces bourgeons adventifs. Dans les Equisetum les bourgeons endogènes des rhizomes donnent des branches latérales vigoureuses qui forment, soit de nouveaux rhizomes, soit des tiges aériennes; tout fragment de rinizome, tout nœud souterrain des tiges dressées est capable de produire une nouvelle plante; quelquefois un entre-nœud de branche souterraine se renfle en tubercule et, à son sommet, un bourgeon terminal peut forme: un chapelet de nouveaux entre-nœuds tuberculeux ou bien se développer en nouveau rhizome. Le Lycopodium Selago produit des bulbilles à la base de ses feuilles. En général, les tiges et les rhizomes ramifiés peuvent se multiplier par destruction des parties anciennes; les branches devenues ainsi isolées et indépendantes constituent autant d'individus différents. Les Cryptogames vasculaires, possèdent donc encore, dans leurs deux générations sexuée et asexuée, une propagation végétative, beaucoup moins développée à la vérité que dans les Mousses.

\section{- CONSIDÉRATIONS GÉNÉRALES SUR LES CRYPTOGAMES VASCULAIRES.}

Entre les Cryptogames vasculaires et les Muscinées, l'analogie est des plus grandes, quand on envisage uniquement les organes de la re- 
produrtion sexuée; elle devient beaucoup plus restreinte, en s'adressant au produit de la fécondation, c'està-dire au sporogone; la romparaison, en effet, n'est plus possible que pour les deux stades extrêmes, les premières divisions de l'embryon d'un côté, la formation des spores de l'autre. Tous les stades intermédiaires, c'est-à.dire tout le système végétatif de la génération asexuée, offre au contraire des différences très notables, plus grandes même qu'entre les Cryptogames vasculaires et. les Phanérogames.

Quand on considère les rapports entre les deux générations sexuée et asexuée, la dissemblance s'accentue encore davantage 'des Muscinées aux Vasculaires. Chez les premières le sporogone est resté parasite du thalle et tout le développement dépend de ce dernier. Les secondes ont su affranchir leur sporogone et en faire une plante adaptée complètement à la vie aérienne. Or, l'air est évidemment un milieu beaucoup plus favorable que l'eau à l'évolution du Règne végétal (MM. de Saporta et Marion, Evolution du règne végétal); les Muscinées, aussi bien que les Cryptogames vasculaires, sont des plantes semi-terrestres, encore sous la dépendance du milieu aqueux pour leur fécondation; les rapports différents entre leurs deux générations sexuće et asexuée rendent certainement cette dépendance beaucoup plus étroite pour les Muscinées que pour les Fougères, où elle n'atteint directement que le seul prothalle. A ce point de vue général, Vasculaires et Muscinées se tronvent dans les mêmes conditions physiologiques, et il faut les considérer comme deux branches divergentes d'un même système dont la base, aujourd'hui disparue, émergeait des Algues. L'une de ces branches n'a qu'une étendue fort restreinte; elle aboutit aux Mousses, qui ont pu s'étendre et se maintenir sur terre grâce surtoul à un retour partiel à la propagation asexuée d'es Algues (protonéma). L'autre branche, au contraire, s'est largement épanouie; elle a conduit à la véritable adaptation à la vie terrestre, en affranchissant finalement la fécondation elle-même du milieu aquatique. Mais, par une conséquence forcée, les rameaux les mieux adaptès de cette branche on fini par iaire disparaitre les rameaux moins perfectionnés, c'est-à-dire moins différenciés de la souche commune; et, en effet, les Cryptogames vasculaires hétérospo- 
rées de la flore actuelle ne sont certes que des débris d'une végétation ancienne. Ces débris offrent des liens de parenté évidents avec les Phanérogames; les Isoètes semblent même plus rapprochées des Monocotylédones, les Sélaginelles des Dicotylédones.

La formation des microspores est identique dans les Hétérosporées et les Phanérogames.

Pour la formation des macrospores, la différence est plus ap parente que réelle (voir plus haut).

T'out se réduit, pour le prothalle, au degré plus ou moins grand de la régression. La lente disparition de cet organe accessoire qui a cessé de se nourrir d'une façon indépendante, s'explique encore par les princıpes d'économie de la nature; les matériaux dépensés pour le prothalle sont perdus, en partie au moins, pour l'embryon; devenu inutile ou même nuisible, cet organe doit finir par être supprimé.

Nous avons démontré l'influence que la régression successive du prothalle exerce sur le premier développement de l'œuf, et établi ainsi, par l'embryogénie, un nouveau lien entre les deux groupes.

La manière d'être de la macrospore est différente; elle se détache dans les Hétérosporées et reste en connexion avec le sporogone dans les Phanérogames. Un état intermédiaire se rencontre encore dans le Ginkgo dont le macrosporange, au moment de la fécondation, est fixé sur le sporogone, mais ne se trouve plus avec lui en connexion intime. Fn outre, des modifications analogues, plus complètes même, se sont déjà présentées dans les sporogones des Mousses qui donnent naissance, sans spores, à la tige feuillée, et dans les individus fructifères ảes Lemanea qui deviennent des branches du protonéma.

Reste donc, en dehors du système végétatif, la fécondation qui est aérienne dans les Phanérogames, tandis que, dans les Cryptogames, elle se trouve sous la dépendance de l'eau. Une spore mâle d'Équiseum, germant dans la goutte de mucilage sur le col de l'archégone, ne pourra y développer son prothalle, ni féconder l'oosphère; dans les Hétérosporées, au contraire, le prothalle mâle est assez réduit pour que la fécondation devienne possible dans ces conditions; de là à l'allongement de l'anthéridie en tube pollinique jusqu'au contact de l'oosphère, à la suppression des anthérozoïdes devenus inutiles, il n'y a 
qu'une gradation successive, toujours conforme à l'économie de la nature. On conçoit très bien que cette marche ait pu être suivie pour transformer, à la longue, la fécondation aquatique en fécondation aérienne. Mais nous ne faisons que déplacer la difficulté; la barrière renversée entre les Phanérogames et les Hétérosporées se dresse de nouveau entre ceux-ci et les Isosporées. C'est ici que les expériences de $\mathbf{M}$. Prantl acquièrent une importance capitale.

M. Prantl a démontré que la prétendue tendance à la diøecie n'existe pas dans les Fougères ; mais qu'on pouvait déterminer physiologiquement, par une nourriture insuffisante, par la privation d'espace et de lumière, la formation de prothalles rudimentaires et mâles; il est vrai que cela n'explique pas l'apparition de prothalles ne portant que des archégones. Nous devons entrer ici dans le domaine des hypothèses : des individus isosporés ont pu fournir des spores inégales; dans les sémis serrés, les plus petites de ces spores ont développé des prothalles rudimentaires et mâles; la fécondation croisée entre les anthérozoïdes de ces derniers et les archégones des prothalles normaux a dû être avantageuse pour la descendance, chez laquelle, par conséquent, la différence entre le volume des spores s'est accentuée; la réserve nutritive, accumulée ainsi successivement dans les grandes spores, a finj par devenir assez considérable pour amener le prothalle, saus nutrition indépendante, à son stade final, correspondant à la for. mation des archégones; ce stade a même pu être atteint plus rapidement, l'assimilation étant plus facile que dans la nutrition indépendante; alors il y a eu tout naturellement condensation dans le développement et suppression du stade intermédiaire des anthéridies qui sont devenues inutiles. Mais, je le répète, ce sont là des hypothèses, et jusqu'à ce que cette difficulté ait été levée, la différence, au point de vue de la reproduction, apparaitra plus grande entre Isosporées et Hétérosporées, qu'entre Hétérosporées et Phanérogames. 


\section{RÉCAPITULATION}

A l'origine, la reproduction ne semble qu'une conséquence d'une nutrition abondante et de l'accroissement; elle se réduit à un fractionnement du protoplasma. Ce procédé se perfectionne par la bipar-fition cellulaire, la formation des zoospores qui propagent l'espèce dans l'espace, la formation des spores durables qui la propagent dans le temps. Puis on voit ces corps agames se fusionner entre eux, d'abord indifféremment au nombre de deux ou de plusieurs, sans qu'entre les éléments qui copulent il soit possible de distinguer aucune différenciation. Cette fusion qui ne semble avoir d'autre résultat qu'une augmentation de masse, est cependant l'origine de la sexua-. lité. Celle-ci s'accentue par des différences de taille, de motilité, et évidemment aussi des différences plus profondes; les deux protoplasmes deviennent complémentaires l'un de l'autre; ils sont d'abord capables de se développer encore isolément, ce qui prouve que la différence est faible; mais ils ne tardent pas à perdre cette faculté. L'élément femelle finit par devenir immobile et dès lors la fécondation a atteint ses caractères apparents essentiels : copulation entre élément mâle mobile et élément femelle immubile. Les Algues présentent le plus grand intérêt au point de vue de ces transforma tions successives.

Pendant longtemps les changements se réduisent alors à des perfectionnements secondaires (col et ventre de l'archégone, cellules du canal, etc.). Mais de nouvelles transformations apparaissent par l'adaptation complète à la vie aérienne; la motilité disparaît alors aussi dans l'élément mâle, de nouveaux auxiliaires viennent remplacer l'eau, et les deux cellules sexuées peuvent obéir à la tendance générale du protoplasme végétal de ne vivre qu' entouré de cellulose.

La fécondation tout d'abord ne paraît remplir qu'une place secondaire; elle est pour ainsi dire accidentelle et déterminée par les con- 
ditions extérieures, le froid, la sécheresse; elle a pour effet de donner une spore durable, la force de résistance du protoplasma étant augmentée, semble-t-il, par la copulation. Mais évidemment cette fusion de cellules différentes est avantageuse pour la descendance et donne une nouvelle impulsion au végétal ; aussi se montre-t-elle bientôt plus fréquemment. Dans les plantes terrestres, elle acquiert une importance beaucoup plus grande que dans la généralité des plantes aquatiques; elle alterne régulièrement avec des spores asexuées qui représentent les carpospores des Algues; toutes les autres générations agames disparaissent (protonéma excepté). Le rôle de la reproduction asexuée se trouve donc de plus en plus réduit; si les progrès modernes de la botanique ont fait retrouver encore dans les Phanérogames les carpospores, celles-ci ont disparu néanmoins physiologiquement. La propagation végétative devieni aussi de moins en moins importante.

En dehors des Champignons où elle se dégrade sous toutes ses formes, la reproduction se perfectionne dans chaque groupe particulier; elle fournit les meilleurs liens entre ces groupes, qu'on a pu, grâce à ces organes, rattacher naturellement les uns aux autres. 


\section{INDEX BIBLIOGRAPHIQUE.}

Arcangeli. - Sulla Pilularia globulifera e sulla Salvinia natans. Nuovo Giornale Bot. Ital., rol. VIII, 1876.

- Sopra alcune specie Batrachospermum. Nuovo Giornale Bot. Ital. vol. XIV, 1882.

Areschong. - Observationes phycologicæ, II et III.

- Copulation des Phéosporées. Botaniska Notiser, 1873.

Arloing, Cornevin, Thomas. - Compt, rend. 1880, 1881, 1882, Bactéries.

Annell. - Observation d'une fécondation chez les Mousses. Botaniska Notiser of Nordstedt, 1875.

Bainier. - Étude sur les Mucorinées. Thèse École de pharmacie, Paris, 1882.

BAlogh: - Medical Wochenblatt, 1876. Microzyma.

Baranetzky. - Entw. gesch des Gymnoascus Reessii. Bot. Zeit., 1872, no 10.

BARY (DE). - Ueber die Keimung einiger grossporigen Flechten. Jahrb. f. w. Bot. vol. V.

- Untersuch. über Brandpilze, etc. Berlin, 1853.

- Neue Untersuch. über Uredineen. Monatsber. der Berl. Akad., 1865.

- Die Mycetozoen. Leipzig, 1864.

- Mirphlologie und Physiologie der Pilze, Flechten und Myxomyceten; II vol, de Handbuch der physiol. Bot. de Hofmeister.

- Ueber die Keimung der Lycopidiac. Berichte der naturf. Ges. zu Freiburg in Br., 1858, H. IV.

- Ueber apogame Farne und die Erscheinung der Apogomie im Allgem. Bot. Zeit., 1878.

- Unters. ïber die Peronosporen und Soprol. und die Grundlagen eines naturl. Systems. der Pilze. Frankf. a M. et Bot. Zeit., 1881.

- Unters. über die Familie des Conjugaten. Leipzig, 1858.

- Ueber den Befruchtungsvorgang bei den Charen. Monatsb., mai, 1871.

- Zur Keimungsgeschichte der Charen. Bot. Zeit., 1875.

- Algues. Bot. Zeit., 1855, 1856, 1857. Volvocinées, id. 1858.

- Ueber Schimmel und Hefe. Berlin, 1867.

- Recherches sur le développement de quelques champignons parasites. Ann. Sc. N., 4e S. XX.

- Ueber die Fruchtentwickl. der Ascomyceten. Leinzig, 1863.

- et Woronine. - Beitr. zur Morph. und Phys. der Pilze. Francfort, 1866.

BART (DE), - Ueber den geschlecht. Zeugungsprozes bei den Algen. Berichte der naturf.

Ges. in Freiburn. vol. I, 1856. 
BARY (DE). - Beiträge zur Kenntniss der Nostocaceen. Flora, 1863.

- und Strasburger. - Acetabularia mediterranea. Bot. Zeit., 1877.

- Sur l'Ecidium Abietinum. Bot. Zeit. Trad. dans Ann. Sc. N.

- Zur Kenntniss insektentoedender Pilze, 1867.

- Ueber Edogonium und Bulbochaete. Frankfort, 1854.

- Zur Systematik der Thallophyten. Bot. Zeit., 7 janv. 1881.

Bastian. - Poced of the Royal Society., 1873.

- Compt. rend. 83. Génération spontanée.

BAuke. - Zur Entw. gesch. der Ascomyceten. Bot. Zeit., 1877, no 20.

- Pycniden. Nova Acta, V. III, 38.

- Beitr. zur. Entw. gesch. der Schizeaceen. Jahrb. f. w. Bot. XI.

- Entw. gesch. des Prothallium bei den Cyatheacen. Jahrb. f. w. Bot.

Béchamp. - Comptes rendus, 83, Microzyma.

Berggren. - Om Azollas prothallium och embryo. Lunds Univers. Arsskrift, t. XVI.

- Germination des propagules. Iakttagelser öfver Mossornas Könlösa fort plantuing.

Lund, 1865 et $18 \% 0$.

- Propagules des Mousses. Lunds Univ. Arsskrift, vol. I.

- Botanika Notiser, 1873 et 1876. Bot. Zeit., 1872.

Berkeley. - Journ, of Linn. Soc., 1864, vol. IIl.

- Voy. Cooke.

- Sur la fruct. du Lycoperdon, du Phallus. Ann. Nat. Hist., 1840, vol. IV.

- et Broомe. - Britsch Hypogœus Fungi. Ann. Nat. Hist., 1849, vol. XYIII.

- Gardeners Chronicle, noor. 1879.

Bert (Paul). - Anaerobies. Compt. rend., 25 férrier 1877.

- Expériences sur la Bactéridie. Soc. de biologie, 1875.

Berthold. - Zur Kenntniss der Bangiaceen Mitth. d. zool. Station zu Noapel. Bı. II.

- Die geschlecht. Fortpflanzung der eigentlichen Phéosporeen, do Bd. II, 1881.

- Zur Kenntniss der Siphoneen, $d^{\circ}$ Bd. II, 1880.

- Die geschlechtl. Fortpfl. von Dasycladus claveformis. Bot. Zeit., 1880.

- Bot. Zeit., 1881, 6 mai. Phéosporées.

Bischoff. - Die Rhizocarpeen und Lycopqdeen. Nurnberg, 1828.

Bochlendorff. - Contribution à la Biologie des Schizomycètes. Dissertatio inauguralis. Dorpat, 1880.

Bommer. - Revue et classification des Cyathéacées, Bull. Soc. Bot. Fr., vol. XX.

Bónnet. - Azolla caroliniana. Bull. Soc. Bot. Fr., 10 juin, 1881.

Bornet. - Rech. sur la struclure de l'Ephobe pubescens. Ann. Sc. Nat, $3^{e}$ s, t. XVIII. - Gonidies des Lichens. Ann. Sc. Nat., 5e s., t. XVII et XIX.

Bornet et Thuret. - Notes algologiques. Paris, 1876.

- Recherches sur la fécondation des Floridées. Ann. Sc. Nat., $5^{e}$ s., vol. VII.

Borodin. - Mélanges biolog. tirés du Bull. de l'Académie impériale des sciences de

Saint-Pétersbourg, T. VI, 1867, p. 538. 
Borzi. - Note alla morfologia e biologia delle alghe Ficocromacee. Nuovo Ciornale Bot. Ital. $\mathrm{X}$ et XI.

- Nuov. Giorn. Bot. Ital. 1878., Sexualité des champignons.

Boszcow. - Les Bacillariacées d'eau douce du sud-ouest de la Russie. Kiew, 1873.

Boutroux. - Sur l'habitat et la conservation deslevûres spontanées. Bull. Soc. Linéenne de Normandie, $3^{\mathrm{e}} \mathrm{s}$., vol. VI, 1881.

Bower. - Development of the conceptacle of Fucacere. Quart. Journ. of. Microsc. Science, t. XX, 1880 .

Braun. - Ueber Marsilia und Pilularia. Monatsb. der Berl. Akad, 1870.

- Ueber Isoetes. Monatsb. der Berl. Akad, 1863.

- Die Verjüngung in der Natur.

Brefeld. - Unters. über die Entw. der Empusa muscæ und Entomopithora radicans, etc. Halle, 1871.

- Unters. über Alkoholgährung. Sitzb. d. physik. med. Gesellsch. zu Wurzburg, 1873.

- Die Entw. gesch. der Ascomyceten. Bot. Zeit., janv. 1876.

- Mycologische Untersuchung. Bot. Zeit., 1877.

- Unters. über die Spaltpilze. Bericht ïber die Sitzung der Ges. naturf. Freunde in Berlin. Bot. Zeit., 1878.

- Sitzungsber. 1. deutsch. chem. Gesellsch. Neue Folge. Bot. Zeit., 7 et suivants pour Saccharomyces.

- Bot. Unters. über Schimmelpilze. Leipzig; 1872-1881.

- Ueber Dictyostelium mucoroides. Abhandl, der Senkenbergische Gesellsch. Frankfurt a, M. VII.

Bruchmann. - Ueber Anlage und Wachsthum der Wurzeln von Isoëtes und Lycopodium, 1874.

Buchner. - Charbon (sang de rate). Sitzber. der k. bayer. Ak. d. W. München, 1880, H. III, et $1882, \mathrm{H}$. II.

Burck. - Over die entwikkelingsgeschiedenis an den aard van het Indusium der Varens. Harlem, 1874.

- Surle développement du prothalle des Aneimia. Archives neerl., X.

BüsGex. - JahrlJ. f. w. Bot., 1882, XIV, pour Develop. des sporanges d. les Phycomycètes.

Carnoy. - Bull. Soc. Bot. de Belgique, 1870, IX, 2. Mucorinées.

CArter. - Annals and Mag. of nat. Hist., 1858, pour fécondation des CEdogonićes.

Chaniberland. - Comptes rendus, 24 mars 1879. Résistance des germes de certains organismes à la température de $100^{\circ}$.

Chatis (Ad.). — De l'anthère. Recherches sur le dévelop., etc. Paris, 1870.

Chauveau. - Comptes rendus, 1881. Bactéries:

Cienkowski. - Das Plasmodium. Aussi : Zur Entw. gesch. der Myxomyceten. Jahrl. f. w. B. III, p. 325 . 
Cienrotvsri. - Morphologie des Ulothrichées. Bull. Ac. des Sc. de Saint-Pétei'sbourg, 1871, t. VIII et XXI, 1876.

- Ueber Palmellenzustand bei Stigeoclonium. Bot. Zeit., 1876.

- Morphol. des Bactéries. Mém. de l'Ac. des Sc. de St-Pétersb, t. XXV, 1877.

- Die Pilze der Kahmhaut (Mycoderma), do, vol, VIII.

Сонм. - Empusa Muscae. Nova Acta, vol. XXV.

- Sphaeropléacées. Ann. Sc. N., $4^{\mathrm{e}}$ série, vol. V.

- Ueher Bau und Fortpflanzung von Volvox globator. Berichte der schles. Ges., 1856. Ann. Sc. Nat., 1857.

- Ueber Chlamydococcus und Chlamydomonas. Ber. der schl. Ges., 1856.

- Pour Pilobulus crystallinus. Nov. Act. Ac. nat. curios, XV.

- Beitræge zur Biologie der Pflanzen

- und Wichura. - Ueber Stephanosphæra pluvialis. Nov. Act. Acad. nat. curios, XXVI.

Cooke et Berkeley. - Les champignons, Paris, 1882.

Corda. - Icones Fungorum.

Cornu. - Bull. de la Soc. Bot. de France, t. XXI, p. 161, pour prothalle et anthéridies de l'Aspidium filix mas.

- De la fécond, chez les Algues et en partic. chez l'Ulothrix seriata. B. de la Soc. Bot. de France, 1874.

- Monographie des Saprolégniées. Ann. Sc. Nat., 5e série, 1872.

- Reprod. des Ascomycètes. Stylospores et Spermaties, 6e série, t. III, 1876.

- Causes qui déterminent la mise en liberté des corps agiles. Compt. rendus, 5 nov.1877.

- Pour champignons et leurs générations alternantes. Bull. Soc. Bot. de France, 18721882.

- Sur la reproduction des Algues marines. Comptes rendus, 89.

Cramer. - Ueber Entstehung und Paarung des Schwärmsporen v. Ulothrix..B. Z., 1871.

- Ceramiaceen. Neue Denkschr. Schweiz. Ges. Bd. XX. Zurich, 186't.

Crié. - Sur les Dépazées. Ann. Sc. Nat, $6^{\circ}$ série, t. VII.

Cunnisgharr. - On Mycoïdea parasitica, a new Genus of Parasitic. Algre. Transact. of the Linn. Soc. of London, $2^{\mathrm{e}}$ sér., vol. I, 1880.

Dallinger et Drysdale. - Monthly Microscopical Journal, septembre 1875. Bactérie

DAVAINE. - Bacillus anthracis. Compt. rend. depuis vol. LVII.

Derbès et Solier. - Mémoire sur quelques points de la physs. des Algues. Suppl. au Compt. rend. de l'Acad. des Sc., 1856.

Dodel. - Ulothrix zonata. Jahrb., Bd. X.

Duclaux. - Ferments et maladies, Paris, 1882.

Dutallly. - Bull. mens. de la Soc. Linéenne de Paris, 7 nov. 1877. Equisétacées.

Duval-Jouve. - Hist. nat. des Equisetum de France. Paris, 1864.

Ehrenberg. - Infusions Thierchen, 1838. Bactériens.

EidaM. - Bot. Zeit., 1875. Recherches sur la fécond. des Agaricus. 
Eidas. - Ueber Pycniden. Bot, Zeit., :877.

- Nidulariées, dans Cohn's Beitrægen, II.

- Pour le dévelop. de l'ascogone des Gymnoascées. Cohn's B.

- Ueber die Entw. des Sphærotilus natans. (Nostoc.) Sitzb. d. b. V. der Provinz Brandenburg, avr. 1879.

Elfing. - Studien über die Pollenkörper d. Angiosp. Jenaisch. Zeitschr., XllI, 1879.

EnGEL. - Conidies du Bacillus puerperalis. Compt. rend., 1879, n 19, Revue méd. de l'Est̂, p. 52 et 159.

- Les ferments alcooliques. Thèse Fac. Sc., Paris, 1872.

Ewart. - On the Life. History of Bacillus Anthracis. Quaterly Journal, avril 1878.

Falkenberg. - Artiçle Algues dans Lehrbuch der Bot. von Schenk.

- Befi'ucht, und Generationswechsel von Cutleria. Mitth. der zool. Stat. zur Neapel, I, 1878.

- Ueber Discosporangium. Mitth. der zooi. St. zur Neapel, Bd. I, 1878.

Famintzin et Baranetzky. - Zur Entwickl, gesch. der Gonidien, etc. Mém. Ac. Sc. St.-Pétersb., XI et B. Z. 1868.

Fainintzin et.Woronin. - Sur deux nouvelles formes de Schizomycètes. Mém. de l'Ac. des Sc. de St-Pétersb., $7^{e}$ sér., vol. XX.

Fankhauser. - Ueber den Vorkeim von Lycopodium. Bot. Zeit., 1873.

FARLow. - Quaterly Journal of microscopial Sc. New. Soc. XIV, 1874. Apogamie.

FEe. - Mérooires sur la famille des Fougères. Strasbourg, 1844-1869.

Fehlner. - Ueber ein verzweigtes Moosporogonium. CEster. Bot. Zeit., Juni 1882.

Fellner, - Keimung der Sporen von Riccia glauca. Jahresb. des aliad. naturiviss Vereins in Graz., 1875.

Fischer. - Beiträge zur Kenntn. des Nostochaceen. Bern, 1853.

- Bot. Zeit., 8 oct. 1880. Olpidiopsis (Chytridinies).

- Unters. über die Parasiten der Saprolegnien. Jahrb. f. w. Bot. 1882, XIII.

Fischer (de Waldheym), - Jahrb. f. w. Bot. VIII, 1869. Ustilaginées.

- Ueber die Eutw, den Farnsporen. Jahrb. f. w. Bot., IV.

- Aperçu systém. des Ustilaginées. Paris, 1877.

- Les Ustilaginées. Varsovie, 1877-1878.

Frisch, - Ueber das Verhalten der Milabrandbacillen gegen extremniedere Temperaturen. Sitzunsber. der Kais. Akad, der W., 1880.

Fuistrng. - Beitr. zur Entw, gesch. der Lichenen. Bot. Zeit., 1868.

- Apothécies. Berlin, 1865. (Dissertatio inauguralis).

- Zur Entw. gesch. der Pyrenomyceten. Bot. Zeit., 1867, 1868.

Gayon. - Mucor circinelloïdes et Mucor spinosus. Compt. rend., 7 janv. 1878.

GEDDES. - Observ. of. the resting state of Chlamydomyxa labyrinthuloïdes. Quarterly. Journal, janv. 1882.

- On the coalescence of amoeboid. cells into plasmodia. Proceedings of the Royal Society, $1880, \mathrm{n}^{0} 202$. 


\section{$-218-$.}

Hofmeister. - Berichte der k. Sächs. Ges. der Wiss., 1854. Mousses.

- Abh. der k. Sächs. Ges. der Wiss., 1855, IV, et Jahrb. III. Equisétacées.

- Entw. von Isoëtes lacustris. Abh. der k. Sächs. Ges. der. Wiss., IV, 1855.

- Abh. der k. Sächs. Ges. der Wiss., 1857. Uphioglossées, Salvinia natans.

- Beiträge zur Kenntniss der Gefässkryptogamen, II.

Hutton. - Observ. on the differ. modific, in the capsules of moosses, etc. Transact. and Proceed of the New. Zeeland Instit,; 1874.

JANCZEwSKr. - Bot. Zeit,, 1871. Discomycètes.

- Ann. Sc. Nat., $5^{\circ}$ série, XVII.

- Mém. de la Soc. Sc. Nat. Cherbourg, XXII, juillet 1872. Porphyıracées.

- Ann. de la Soc. Sc. Nat. Cherbourg, XVIII, 1872. Les propagules du Sphacelaria cirrhosa.

- Recherches sur l'ascobolus furfuraceus, 5e série, t. XV, 1872.

- Développement des cystocarpes dans les Floridées. Mém. de la Soc. des Sc. Nat. Cherbourg, 1876.

- Vergleich. Unters. über die Entw. gesch. des Archegoniums. Bot. Zeit., 1872.

- Observat. sur la reprod. de quelques Nostochacées. Ann. Sc. Nat., 5e série, t. XIX, 1874.

JANCZEwsir et Rostafinski. - Observ. sur quelques algues possédant des zoospores dimorphes, Mém. Acad. Cherbourg, t. XIX, 1874.

- Note sur le prothalle de l'Hymenophyllium Tunbridgense. Mém. Soc. Sc. Nat. Cherbourg, 1875.

Jonkmann. - Compte rendu de l'Acad. des sciences d'Amsterdam, 1875, et Bot. Zeit., 1878, pour Prothalle des Marattiacées.

- Actes du Congrès intern. des Botanistes, etc. Amsterdam, 1877.

Jurany. . - Bot. Zeit., 1871, pour Sporanges de Psilotum.

- Beiträge zur Morph. der Edogonien. Jahrb. f. w. Bot., XIX, 1873.

- Ueber die Entw. der Sporangien und Sporen von Salvinia natans. Berlin, 1874.

- Bot. Jahresbericht, 1875, pour Sporanges de Tmesipteris.

Kansten. - Das Geschlechtsleben der Pflanz. und die Parthenogenesis. Berlin, 1860.

- Bot. Unters. aus dem physiol. Labor, etc. Berlin, 1866, B. I, H. II.

KERN. - Bot. Zeit., 1882. Spores des Bactériens.

Kıскx. - Bot. Zeit., 1871, pour Sporanges de Pilotum et de Tmesipteris.

Kienitz-Gerloff. - Vergl. Unters, über die Entw. gesch. des Leebermoossporogoniums. Bot. Zeit, 187.4.

- Neue Beiträge zur Entw. gesch. des Leebermossporogoniums. Bot. Zeit., 1875.

- Ueber den genet. Zusammenhang der Moose mit den Gefässskrypt. und Phanerog. Bot. Zeit., 1876.

- Unters. über die Entw. gesch. der Laubmooskapsel und die Entwickl. ciniger Polydiaceen. Bot. Zeit., 1878.

- Bot. Zeit., 2 décembre 1881, pour Prothalle femelle des Isoëtes et Embryogénie. 
Kirchner. - Beobacht. der Geschlechtsorgane bei der Gattung Coprinus. Sitzber. der schles. Gesell. f. vaterl. Cultur., 11 février 1875.

Kuellanan. - Beitrag till Künnedom om Sliandinaviens Ectocarpeeroch Tilopterideer. Stockholm, 1872.

- Ueber die Algenveget. des Murmannschen Meeres. Nov. Acta Reg. Soc. Scien. Upsalis, 1877.

KLebs. - Beiträge znr Kenntn. niederer Algenformen. Bot. Zeit., 1881.

KLEIN. - Ueber Vampyrella. Bot. Zeit., mars 1882.

- Propagules de Marchantia. Bot. Centralblatt, vol. V, no 1.

KNY. - Beitr. zur Entw. gesch. der Farnkräuter. Jahrb. f. wiss. Bot., Bd. VIII.

- Die Entw. der Parkeriaceen, dargest. an Ceratopteris thalictroïdes Leop. Carol. Akad., Bd, XXXVII.

- Sitzunsb. der Geș. naturf. Freunde. Berlin, 1871. Chytridinées.

- Entw. der laubigen Lebermoose. Jahrb. IV.

- Entw. der Riccien. Jahrb. V.

- Ueber Axillarknospen bei Florideen, 1873.

KocH. - Dans Beiträge de Cohn, 1877. Bactériens.

- Ueber Tuberculose. Deutsche médec. Wochenschrift.

Krabвe. - Formation des apothécies des Lichens. Bot. Zeit., février 1882.

K̈̈нn. - Mitth. aus dem Gebiet der Bot. von Schenk und Luerssen, I, Andréacées.

- Unters. über die Entw. etc. des Mutterkorns. Mitth. aus dem physiol. Labor. Halle H. I, 1863.

Lantzius Beninga. - Beiträge zur Kenntn. des Baues der ausgew. Mooskapsel. Nov. Act. Acad. Leop., 1856.

LEITGeb. - Studien über Entwicklung der Farne.

- Sitzunsber. der Wiener Akad., 1868, 1869, pour Mousses, et, en général, toute la série.

- Sitzunsber, der w. Akad., 1871, LXIII. Bot. Zeit., 1871-1872, pour Hépatiques.

- Die Entw. der Iiapsel von Anthoceras. Sitzber. d. Ak. d. W. Wien, vol. LXXIII, 1876.

- Ueber verzwergte Moosporogonien. Mitth. der naturw. V. für Steiermark, 1876.

- Zur'Embryologie der Farne. Akad. der Wiss. zu Wien, mars 1878.

- Das Sporogon von Archidium. Sitzunsber. der W. Ak. Bd. LXXX, nov. 1879.

- Unters. über die Lebermonse Gratz, 1874-1881.

- Jahrb. f. wiss. Bot, VII. Saprolégniées.

Le Monnier, - Voir Van Tieghem.

Lorentz. - Bau und Entw. gesch. der Laubmoose. Leipzig, 1864.

- Zur Anat. und Lntw. gesch. von T'immia austriaca. Bot. Zeit., 1867.

Luders. - Beobachtungen ïber Organisation, Theilung und Copulation der Diatomeen. Bot. Zeit., 1862.

Ludwig. - Génération de conidies dans Polyporus Ptychogaster nov. spec. Zeitschr. f. d. ges. Naturw., mai-jụi 1880. 
Lierssen. - Zur lieimungsgesch. der Osmundiaceen, vorzüglich der Gattung Todea. Mitth. aus d. Gesammtgeb. d. Bot. de Schenk et Luerssen.

Magnin. - Les Bactéries. Paris, 1878.

Marchand. - Botanique cryptogamique. Paris, 1882.

Marion. - V. Saporta (de).

Miattirolo (Oreste). - Nuov. Giorn. bot. Ital., rol. XIV, oct. 1881. Lichen formé pal. un champigncn bazidiomycète.

Maupas. - Compt. rend., 16 juin 1875. Sur la position systématique des Volrocinées.

Mayer (Ad.). - Sitzunsb. der deutsch. chem. Gesellsch. Neue Folge. Bd. VII et suivants. Saccharomyces.

Mer. - Du dével. des sporanges et des spores dans l'Isoëtes lacustris. Comp̧t. rend., janv.-févr. 1881 et Bull. Soc. Bot. France, 25 mars 1881.

MıкA. - Magyar Növénytani Lapok, Klausenburg, 1880, IV, nº 48.

Milde. - Osmundacées. Bot. Zeit., 1868, 1870.

- Monographia Equisétorum. Nova Acta, XXXII.

Millandet. - Le prothallium mâle des cryptog. vascul. Strasbourg, 1869.

- Mém. de la Soc. des Sc. Nat. de Strasbourg, VI, 1868, pour Phycoxanthine, etc.

Mrquel. - Rech. microsc. sur les Bactéries. Annuaire de Montsouris, 1882.

Mrriec. - Sur le Marchantia. Mém. de l'Acad. des Sc. de Paris, XIII, 1835.

Montagne. - Multiplic. des Charagnes par division. Ann. Sc. Nat., 1852, XVIII.

Montagne et Borx. - (Hépatiques). Ann. Sc. Nat., $3^{e}$ série, vol. I.

Muggenburg (von). - Doppel Fructification von Polyporus aplanatus. Oester. ijot. Zeitschr., oct. 1880.

Müller. - Entw. gesch. der Kapsel von Ephemerum. Jahrb. YII.

NäGELI. - Neuere Algensysteme. Neuenberg, 1847.

- Gattungen einzelliger Algen. Zurich, 1849.

- Beiträg. zur Morph. und. System. der Ceramiacere. Sitzunsb. d. k. B. Ak. d. W. II, 1861.

- Zeitsch. f. wiss. Bot. pour prothalle et anthẻridies des Fougères, et pour Hépatiques, Mousses.

- Pflanzenphys. Unters. H. I, pour Mousses. H. III, Lycopodium Cramer.

Nordstedt. - Characées. Lunds Univers. Arsskrift., II, 1866.

- und Wahlstedt. - Germination des Characées. Flora, 1875.

Nowakowskr. - Die Copulation bei einigen Entomophthoreen. Bot. Zeit., 1877.

- Chytridinées. Cohn's Beitr. II.

Uerstedt. - Extr. des Actes de la Soc. roy. danoise des Sc. nat., janv. 1865. Copenhague, pour la fécond. des Hymenomycètes.

Pasteur. - Pour Bactéries et fermentations. Compt. rend. depuis 1860.

- Sur les corpuscules organisés de l'atmosphère. Ann. Ch. et Physique, $3^{\ominus}$ série, t. LXIV.

- Atténuation de la virulence. Journ. Pharm. et Chim.; 5º́r., t. III, 1881. 
Pasteur. - Études sur la bière. Páris, 1876.

- Compt. rend. LXXXV, 1877; LXXXVI, 1878, Septicémie. T. LXVI, Micrococcus bombycis.

Pasteur, Joubert et Chamberland. - Compt. rend. depuis 1860.

Patoulllard. - Sur l'appareil conidial du Pleurotus ostreatus. Bull. Soc. Bot. Franc., t. XXVII, 1880.

Petit. - Observ. critiques sur les genres Spirogyra et Rhynchonema. Bull. de la Soc. Bot. de France, 1874.

- Note sur le trichogyne de l'Hildebrantia Rivularis. Bull. Soc. Bot. Fr., 25 juin 1880. Pfeffer. - Die Entw, des Keimes der Gattung Selaginella. Bot. Abh. vou J. Hanstein, H. IV, 1871.

Pfitzer. - Unters. über Bau und Entw. der Bacillariaceen (Diatomées). Bonn, 1871.

- Ancylistes Closterii. Monatsb. der Berl. Akad., 1872.

- Article: Bacillariaceen, dans Lehrbuch der Botanik von Schenk. Breslau, 1882.

Plowright. - Grevillea, t. IX, 1880. Diffusion des spores dans les Elvellacées.

Prantl. - Bemerk. über Verwandtschaftsverhältnisse der Gefässkryptogamen, etc. Verhandl. d. physikol med. Ges: zu. Wurzburg, X.

- Die Entw. gesch. des Prothall. von Salvinia natans. Bot. Zeit., 4 juillet 1871.

- Unters. zur Morph. der Gefässkrypt. Leipzig, I, 1875-1881.

- Flora, 1878, pour le développement du prothalle:

- Beobacht. über die Ernährung der Farnprothallien und die Vertheilung der Sexualorgane. Bot. Zeit., nov. 1881.

Praznowski. - Unters, über die Entw. gesch. und Fermentwirkung einiger Bacterien Arten. Leipzig, 1880.

Prilleux. - Sur la formation et la germination des spores des Urocystis. Bull. Soc. Bot. Fr., 25 juin 1880.

Pringsheim. - Characées. Jahrb. f. w. Bot. III.

- Ueher Keimung der ruhenden Sporen bei Spirogyra. Flora, 1852.

- Monatsb., 1855 et Ann. Sc. Nat., $4^{\mathrm{e}}$ sér., 1855, pour fécond. de Vaucheria.

- Morpholog. der Edogonien. Jahrb. f. wiss. Bot., I, 1855. Ann. Soc. Nat., 4e sér ., 1856.

- Ueber die Dauerschwärmer des Wassernetzes. Monatsb., XIII, déc. 1860. Traduit Ann. Sc. Nat., $4^{e}$ sér., t. XIV,

- Ueber Paarung von Schwärmsporen. Monatsb., oct. 1869.

- Monatsb. 1857 et Jahrb. I, pour reproduction des Saprolégniées, do Jahrb. IX, 1873.

- Sphacelariées. Abhandl. der Berl. Akad., 1873.

- Entw. gesch. des Achlya prolifera, Nova Acta. Ac. Car. XXIII.

- Ueber Sprossung der Moosfrüchte und den Generationswechsel der Thallophyten. Jahrb. . w. Bot., XI.

- Ueber Befruch. und. Keimung der Algen. Jahrb. f. w. Bd. II, p. 470.

- Beiträge zur. Morph. und Syst. d. Algen. Jahrb. Bd. II. 
Pringsiem. - Bryopsis. Monatsb. de Berl. Akad., 1871:

- Ueber reget. Sprossung von Moosfrucht. Monatsb. d. Berl. Ak., 1876.

- Zur Morphol. der Salvinia natans. Jahrb. f. wiss. Bot. III, 1863.

Rathax. - Ueber Spermogonien der Ecidiomyceten. Sitzb. d. k. Akad. d. W. 10 juin 1880 .

R.iuwenhoff. - Compt. rend. de l'Acad. des sciences d'Amsterdam, 1877 et 1879, pour dévelop. du prothalle des Gleicheniacées:

REEs. - Entw. der Polypodiaceensporangiums. Jahrb. V, 1866.

- Bot. Unters, über die Alcoholgähriungspilze. Leipzig, 1870.

- Sitzber. der phys. med. Soc. in Erlangen décembre 1874. Fécondation des Basidiomycètes.

- Rechtfertigung. Bot. Zeit., 1876. Fécond. des Basidiomycètes.

- Ueber den Soorpilz (Saccharomyces albicans). Sitzb. der phys. medic. Societ. zu Erlangen, 1877.

Reess. - Die Enstehung der Flechte Collema. Monatsb. d. k. Akad. d. IV., oct. 1871.

- Ueber den Befr. vorgang bei den Basidiomyceten. Jahrb. f. w. Bot. X, 1875.

Rенм. - Die Entw. gुesch. eines die lileearten zerstörenden Pilzes (Peziza ciborioüles). Göttingen, 1872 .

Reinhardt, - Die Copulation der Zoosporen bei Chlamydomonas pulvisculus. Publicat. de la Soc. des sc. nat. de l'Un. de Charkoff, t. X, 1876.

Reinke. - Das TVachsthum und die Fortpflanzung ron Zanardinia, ete. Monatsb. d. Berl. Akad. d. Wiss., oct. 1876.

- Die Cutleriaceen des Golfes von Neapel. Nov. Act. Leop. Ac. d. N. B. XI, no 2.

- Ueber die Entw. von Phyllitis, Scytosiphon und Asperococcus. Jahrb. f. w. B., no 11, 1878.

- Geschlechtspflanzen von Bangia fusco-purpurea.

- Archiv. f. mikr. Anat. von Schultze, V, pour Saprolégniées.

- Entw. gesch. Unters, über die. Dictyotaceen des Golfes von Neapel. Nov. Act. Ac. Leop. Carol., vol. XL, 1878,

- Ueber Monostroma bullosum und Tetraspora lubrica. Jahrb. XI, 1878.

REinsch. - Beobacht. über einige Saprolegnien, Jahrb. f. w. Bot. 1878, t. XI.

- Propagules des Mousses, Linnæa, t. XXIX.

Renault. - Compt. rend., t. LXXXXIV, 1882. Équisétacées hétérosporées.

Renner. - Ustilaginées. Földmüvelezi Erdekeink, 1879, nº 16.

Roвin. - Hist. nat. végét parasit.

Rosanoff, - Rech. anat. sur les Mélobésiées. Mém. de la Soc. des Sc. nat. Cherbourg, VII, 1866.

Rostafinski. - Beobacht. über Paarung von Schwärmsporen. Bot. Zëit., 1871.

- Observ. sur quelques algues possédant des zoospores dimorphes. Mém. Soc. Sc. Nat. Cherbourg, t. XVIII, 1874.

- Versuch eines Systems der Mycetozoen. Berlin, 1873. 
Rostafinsiri. - Quelques mots sur l'Hrematococcus lacustris. Mém. Soc. Sc. Nat. Cherbourg, t. XIX, 1875.

Rostafinsiki und Woronin. - Ueber Botrydium granulaum. Bot. Zeit., 1877. Rostrup. - Ueber eine genetesche Verbindung zwischen Puccinia Molinire et Ecidium Orchidearum.

Roze. - Les Antherozoüdes des Cryptogames. Ann. Sc. Nat., $5^{\ominus}$ sér., t. VII, 1867.

- Des myxomycètes et de leur place dans le Syst. Bull. Soc. Bot. France, 1873, p. 320-336.

Rue (E. DE LA). - Sur le développement du Sorastrum spinulosum. Ann. Sc. Nat. $3^{e}$ sér., t. XV.

- Bull. de la Soc. impér. Sc. Nat. Moscou, 1873. Germ. des Saprolégniées.

Russow. - Beit. zur Kenntn. der Torfmoose (Sphagnacées). Dorpat, 1865.

- Histologie und Entw. d. Sporenfrucht. von Marsilia. Dorpat., 1870.

- Rech. compar. sur les Cryptog. vasculaires. Saint-Pétersbourg, 1872.

Sachs. - Morphologie des Crucibibulum vulgare. Bot. Zeit., 1855.

- Arbeit des bot. Inst. zu Wurzburg. Bd. II, pour le principe du cloisonnenient rectangulaire.

- Traité de Bot., trad. en français par Ph. Van Tieghem, Paris, 1874.

- Traité de Bot., $4^{\ominus}$ édition allemande.

Sadebeck. - Pythium Equisêti dans Cohn's Beit. zur Biolog. der Pffanz., t. I.

- Ueber die Eutw. gesch. der Prothallien und die Embryologic der Schachtelhalme. Bot. Zeit., 1877. Jahrb. f. w. Bot. XI. Sitzb. d. Ges. naturf. Fr. zu Berlin, 1875.

- Article: Gefässcryptog., dans Lehrbuch der Botanik von Schenk, vol. I. Breslau, 1882. Saporta (DE) et Marion. - L'évolution du règne végétal, Paris, 1881.

Sснаснт. - Linnæa, 1849, pour prothalle et anthéridies des fougères.

- Die Spermatozoïden im Planzenreiche. Braunschweig, 1864.

Schenk. - Entw. der Fortpflanz.-Organe und Befrucht. von Vaucheria Geminata. Verh. der. phys. med. Gesellsch. z. Wurzburg, t. VII, 1858.

- Lehrbuch der Botanik, vol. I et II. Breslau, 1881-1882.

Schimper. - Rech. anat. et physiol. snr les Mousses. Strasbourg, 1848.

- Versuch. einer Entw. gesch. der Torfmoose. Stuttgard, 1858.

- Synopsis muscorum europæorum, $2^{\circ}$ éd., 1876.

Schleiden. - Zeitschr. f. wiss. Bot., pour Hépatiques.

Schмiтz. - Halosphæra viridis. Mitth. der zool. Stat. Neapel', t. I, 1878.

- Beobach. über die vielkernigen Zellen der Siphonocladiaceen. Festschrift der naturf. Ges. zu Halle, 1879.

- Fruchtbildung der Squammarien. Sitzl, der Niederrh. Ges. f. Nat. und Heilkunde. Bonn., août 1879.

- Bot. Zeit., 1872, pour formation des auxospores.

- Ueber die Bildung der Sporangien bei der Gattung Halimeda, Sitzber d. Niederrh. G. d. Nat. und Heilk, 1881. 
Scrmutz. - Sitzunsb, der Niederrh. Ges. für Natur, und Heilliunde zu Bonn., 13 juillet 1880, pour format. des anthérozuïdes.

Schröter. - Entw. gesch, einiger Rostpilze. Coln's Beit., t. I, H. III.

Schutzexberger et Destrem. - Fermentations. Compt. rend., LXXXViII.

SCHWEndener. - Unters. über Flechtenthallus. Beit. zur Bot. H. IT, III et IV.

- Ueber die Entw. der Apothecien von Cœnogonium. Flura, 1862.

- Ueber Ephebe pubescens. Flora, 1863.

- Apothécies des Lichens. Flora, 1864.

Seyxes (DE). - Mycoderma. Compt. rend, LXXII et Ann. Soc. Nat., $5^{e}$ sér., t. X.

- Compt. rend., 1er arril 1878. Les Conidies de Polyporus sulfureus.

- Essai d'une Flore mycologique des environs de Montpellier.

Sirodot. - Ét. anat. etc. sur les Lemanéacées. Ann. Sc. Nat., $5^{\text {e }}$ sér.. t. XVI, 1872.

- Compt. rend., 12 mai et 2 juin 1873, pour Batrachospermum.

- Bull. de la Soc. Bot. de France, t. XXII, 1875.

- Le Balbiana investiens. Ann. Sc. Nat., $6^{e}$ sér., t. III, 1876.

- Compt. rend., 1880, p. 862-64. Batrachospermum.

Sollmann. - Bot. Zeit., 1864. Fécondation des Ascomycètes.

Solms-Laubach (DE). - Bot. Zeit., 1867, pour Batrachospermum.

- Fauna und Flora des Golfes ron Neapel und der angr. Meeresabschnitte, herausgeg, von d. zool. Stut, zu Neapel, IV. Monographie, 1881.

Sorokine, - Dévelop. du Scleroderma verrucosum. Ann. Sc. Nat., 6e sír, t. III, 1876.

- Bursulla crystallina, nouveau genre de Myxomycètes. Ann. Sc. Nat., 6e sér., t. III, 1876.

- Dévelop. de l'Aphanomyces stellatus. Ann. Sc: Nat., 6e sér., t. III, 1876.

- Note sur les végétaux parasites des anguillules. Ann. Sc. Nat., $6^{\circ}$ sér., t. IV, 1870.

- Quelques mots sur l'Ascomyces polysporus. Ann. Sc. Nat., $6^{\circ}$ sér., t. IV, 1876.

- Entomophthora dans Cohn's Beiträge, $t$ II.

Spring. - Monog. de la famille des Lycopodiacées. Mémoire de l'Académie royale de Belgique.

StaHL. - Beiträge zur Entw. gesch. der Flechten. H. I et II.

- Ceber künstlichherrorgerufene Protonemabildung an den Sporogon. der Laubmoose. Bot. Zeit., 1876.

- Ueber die Ruheständte àer Vaucheria geniata. Bot. Zeit., 1879.

Strasburger. - Die Befrucht, bei den Farnkräutern. Jahrb. f. w. Bot., VII, 1869.

- Ueber Azolla. Jena, 1873.

- Einige Bemerk. über Lycopodiaceen. Bot. Zeit., 1873.

- Geschlectsorgane und Befrucht. bei Marchantia. Jahrb. VII.

- Studien über Protoplasma. Jena, 1876.

- Ueber Befruchtung und Zelltheilung. Jena, 1878.

- Angiospermen und Gymnospermen, 1879, t. XV et XXIV:

- Zellbildung und Zelltheilung, [3e édition, 1880. 
Suringar. - De Sarcine. Leuwarden, 1865, Archiv. néerland., 1866. Bot. Zeit., 1866. Thunet. - Rech. sur les org. locom. des spores des Algues. Ann. Sc. Nat., $2^{2}$ sér., t. XIX, 1843.

- Note sur la reprod. du Nostoc verrucosum. Ann. Sc. Nat., 3e sér., t. II, 1844.

- Recherches sur les zoospores des Algues et les anthéridies des cryptogames. Ann. Sc. Nat., 3e sér., t. XIV, 1850 et XVI, 1851.

- Observat. sur la repı od. de quelques Nostochinées. Mém. Soc. Sc. Nat. Cherbourg, t. V, 1857 .

- Rech. sur la fécond. des Fucacées. Ann. Sc. Nat., 1854-1855 et Sc. Nat. Cherbourg t. V, 1857.

- Fécondation des Fucacées. Ann. Sc. Nat, $4^{\circ}$ sér., t. II.

- Rech. sur les anthéridies des Algues. Ann. Sc. Nat., 4e sér., t. III.

- Essai de classification des Nostochinées. Ann. Sc. Nat., 6e sér., t. I, 1875.

- Études phycologiques. Paris, 1878.

Thuret et Bonnet. - Rech. sur la fécondation des Floridées. Ann. Sc. Nat., 5e sér., t. VII, 1867.

Tichomiroff. - Peziza Kauffmanniana. Bull. Soc. Bot. de Moscou, 1868.

Toussaint. - Bactéries. Compt rend.; 86 et suivants.

Traube. - Saccharomyces. Sitzb, der deutsch. chem. Gesell. Neue Folge. Bd. VII et suivants.

'Trécul. - Bacillus amylobacter. Compt. rend., 61 et 65.

Tschistiakoff. - Notice prélim. sur l'hist. du dévelop. du sporange et spores de l'Isoëtes Duriaei. Nuov: Gior. Bot. V.

- Matériaux pour servir à l'histoire de la cellule végétale. Rech. anat, et physiol. Ann. Sc. Nat., t. XIX, 1874.

Tulasne. - Fungi hypogaei. Paris, 1851.

- Selecta Fungorum Carpologia, 1861-1865.

' - Dévelop. du Clathrus. Explor. scient. de l’Algérie, p. 434.

- Champignons. Ann. Sc. Nat,, 2e sér., t. XVII et XVIII; $3^{\ominus}$ sér., t. I, IV, VII, XV$\mathrm{XX}$; $4^{\mathrm{e}}$ sér., t. II et III; $5^{\mathrm{e}}$ sér., t. IV, VI, VII, XV.

Thwartes. - The Anuals and Mağ. of. nat. Hist., juillet 1847. Diatomées.

Tyndall. - Les Microbes. Traduction française, Paris, 1882.

Van Tieghem. - Traité de botanique. Paris, 1882.

- Comptes rendus, 8 février 1875 (Coprinus) - do 1878 (Bacillus amylobacter).

- Ann. Sc. Nat., $5^{e}$ sér., t. VIII (Fermentation gallique); $-5^{\mathrm{e}}$ sér., t. XVII (Mucorinées, en collaboration avec M. Lemonnier); $-6^{e}$ sér., t. I (Mucorinées); $-6^{\mathrm{e}}$ sér., t. II (Prétendue sexualité des Basidiomycètes et Ascomycètes); $-6^{\circ}$ sér., t. IV (Mucorinées); $-6^{e}$ sér., t. VII (Leuconostoc).

- Annales scientifiques de l'École normale, 1864 (ferment de l'urée et de l'acide hippurique. 
Vav Tiefriem, - Bulletin de la Sne. Bint. de France, 1877 (Divelop. des Ascomyeites. Aspergillus, Sterigmatocystis, $\Lambda$ scoclesmis, Penicillium aureum).

- Jullotin de la Suc. Bot de France, 1879 (Bactéries, Spirillum, Spirochacte, Pacterinm lucens).

- Bulletin de la Soc. Bot. de France, 1880 (Dimystax Perrieri, Bactiries agrígries, Bactériacées à chlorophylle).

- Bulletin de la Soc. Bot. de France, 1880 (Volvocinée nouvelle répourvue de chlorophylle).

- Bulletin de la Soc. Bot. de France, 1880 (Myxomycètes à plasmode agrégé).

- Bull. de la Soc. Bot. de France, 1881 (Résistance des Bactéries aux hautes températ.). Vaucher. - Histoire des conserves d'eau douce, contenant leurs différents modes de reproduction. Genève, an XI.

Vitradini. - Monographia Tuberaceorum. Miland, 1831.

- Monographia Lycoperdineorum. Mem. delle Ak. Torino, t. V, 1842.

Vouk. - Die Entw. des Sporogoniums von Orthothrichum. Sitzb. d. IV. Akad. B. LXXIII. Abth., I, 1876.

- Die Entw. des Embryo von Asplenium Sheperdi. Sitzl. d. WV. Akad., juillet 1877.

Vriese (DE) et Hartig. - Monographie des Osmundacées, 1853.

WValdner. - Die Entw, des Antheridium's von Anthoceros. Sitzb. der Wiener Ak. del WV., 1877.

- Zur Entw. gesch. der Sporogonion von Andrea und Sphagnum. Bot. Zeit., 1879.

Walz. - Beiträge zur Kentniss der Saprolegnien. Bot. Zeit., 1870.

- Morphologie und System. der Gattung Vaucheria. Jahrb., V.

TVarming. - Untersuch. ïher Pollenbildende Phyllome und Kaulome. Bot. Abh. ron Hanstein, II, H. 2, 1873.

- Videnskabelige Meddelelser fra ren naturhistoriske Forening i Kjöbenhavn, 1875 (Bactéries).

- Unters. und Bemerk. zu den Cycaden K. D. Vidensli. Selsk. Forsh., 1877.

Wartmann. - Beitr. zur Anat. und Entw. gesch. der Lemanea. St-Gall, 1854.

WIGand. - Morph, des genres Trichia et Arcyria. Ann. Sc. Nat., $4^{\mathrm{e}}$ sór., t. XVI.

Wille. - Om Sraermeellerne og deres Copulation hos Trentepollia. Bot. Notiser, 1878.

WVinter. - Puccinia et Ecidium, Sitzb. d. naturf. Ges. Leipzig, 1874 et 1875.

- Lichens. Sitzb. d. naturf.Ges. zu. Leipzig, 1875; Flora, 1875. Jahı.b. f. w. Bot. V, X.

Witrrock. - On the Spore Formation of the Mesocarpese. Svensk. Akad, Handling, Bd. V. Stockholm, 1878.

IVoLF. - Der Brand des Getreides. Halle, 1874.

- Keimung der Ascosporen von Erysiphe graminis. Bot. Zeit., 1874.

IVoronine, - Voyez de Bary.

- Rech. sur les Algues mar. Acetabularia et Espera. Ann. Sc. Nat, qe sér., t. XVI.

- Sur les gonidies de Parmelia pulverulenta. Ann. Sc. Nat., 5e sér., t. XVI.

- Urédinées. Bot. Zeit., 1872 et B. Soc. Sc. Nat. Saint-Pétersbourg, 1874. 


\section{$-227-$}

Woronine. - Plasmodiophora Brassicæ. Jahrb. f. w. But., 1878, t. XI.

- Vaucheria. Bot. Zeit., 1879. 1880.

Wortuington Smitu. - Repr. Agaricinées. Gardner's Chron., 1875.

Zaccharias. - Ueber die Spermatozoïden. Bot. Zeit, déc. 1881.

Zanardini. - Iconogr. med. adriat.

Zops.:- Zur Entw. gesch, der Ascomyceten. Nov, Ac. d. Leop. Carol. Ak., vol. XLII.

- Conidienfrüchte von Fumago, do, vol: XL.

- Chaetomium et Sordaria. Sitzb. d. bot. 'V. Prov. Brandenburg, 1877.

- Polymorph. des Bactéries. Monatsb. d. Berl. Ak., mars 1881.

- Dóhiscence des asques. Sitzb. d. Ges. naturf. Fr. Berlin, 1880. 


\section{TABLE DES MATIÈRES.}

Introduction $\ldots \ldots \ldots \ldots \ldots$

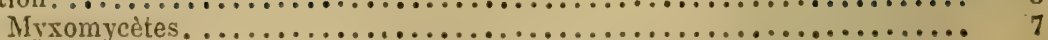

Schizomycètes ou Bactéries................................ 9

Saccharomycètes.................................... 21

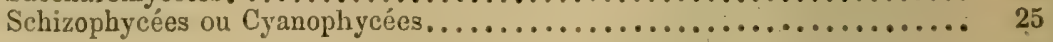

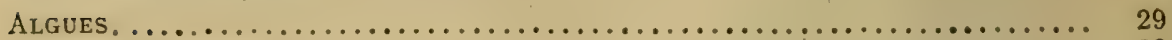

Conjuguées. ............................................. 32

Diatomées.................................................. 36

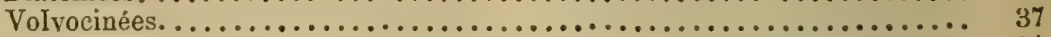

Protococcacées........................................ 41

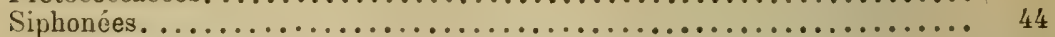

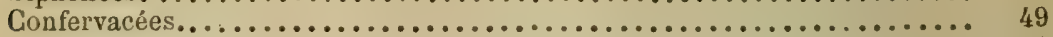

Characées. .......................................... 56

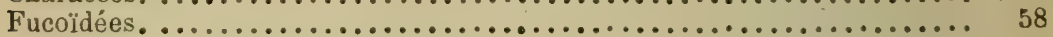

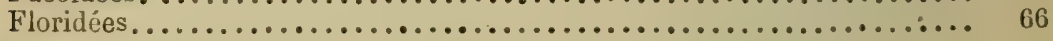

Champignons. ............................................ 74

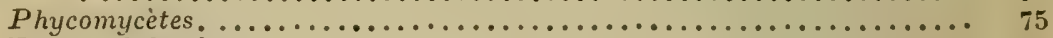

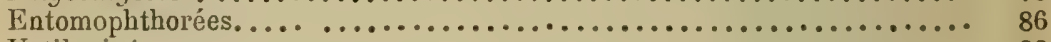

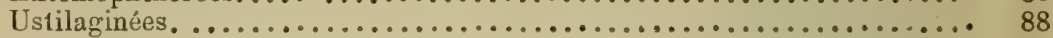

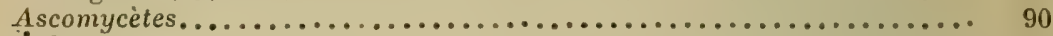

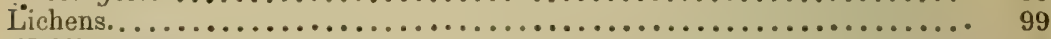

Ecidiomycète: ....................................... 106

Trémellinées........................................ 107

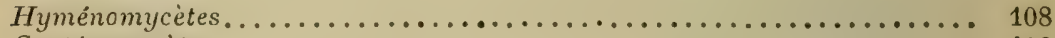

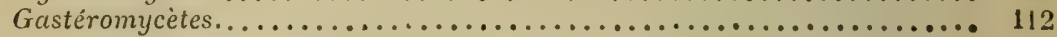

MuscinéEs. .......................................... 113

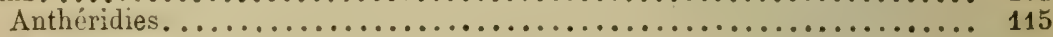

Archégones. .......................................... 116

Mode de distribution des organes sexuels..................... 119

Développement du sporogone.......................... 122

Germination des spores................................. 128

Propagation végétative................................ 131

Cryptogames vasculaires............................... 135

Sporanges.......................................... 137

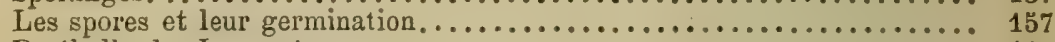

Prothalle des Isosporées.............................. 161

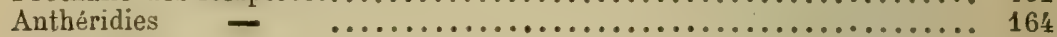

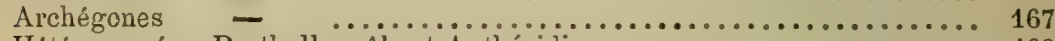

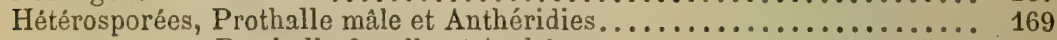

- Prothalle femelle et Archégones........................... 171

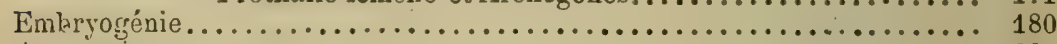

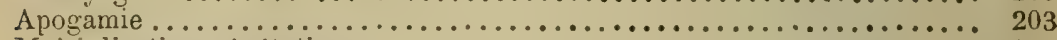

Multiplication végétative................................ 205

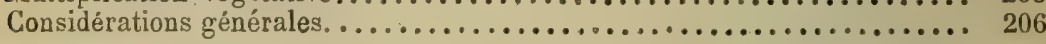

RÉcapitulation....................................... 210

Index bibliographique.................................. 212

PARIS, - A. PARENT, A. DAVY, Sr, IMPRIMEUR DE LA FACULTÉ DE MÉDECINE 3l, RUE MONSIEUR-i.E-PRINCE. 



\section{IIBRAIRIE GERMER BAILLIĖRE ET Ci"}

108, boulevard SAint-Germain.

BOCQUILLON. Manuel dhistoire naturelle médicale. 1871 . 1 vil. in-18 avec 415 fig. dans le texte. 1i $\mathrm{fr}$.

COOKE ET BERKELEY. Les Champignons, avec 110 figures dans le terte. 1 vol. in-8 de la Bibliothèque scientifque internationale. 1875̃. Cartonné. if fr.

HUXLEY. La physiographie, introduction a l'étude de la nature. 1 vol. in-8 avec 128 figures dans lc texte, et 2 planches hors texte (1882). 8 fr. Relié.

$11 \mathrm{rr}$.

LUBBOCK. L'homme préhistorique, étıdié d'après les monument et les costumes retrouvés dans les différents pays de l'Europe, suivi d'une descrintion comparée des mœurs des sauvages modernes, traduit de l'anglais par Ed. Barbier, avec 256 figures intercalées dans ie texte. 1876, ae ídit., augmentée d'une conférence de M. Bnoca sur les Troglodytes de la Vézère. 1 vol. in-8, broché:

Relié

$15 \mathrm{fr}$.

$-18 \mathrm{fr}$.

LUBBOCK. Origine de la civilisation, élnt frimitif de l'homme et mœurs

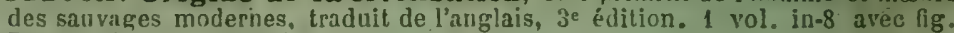
Broché, 15 fr. - Relié.

$18 \mathrm{fr}$.

PISANI (F.). Traité pratique d'analyse chimique qualitative et quantitative, à l'usage des laboratoires de chimie. 1 rolume. in-12. 1880 $3 \mathrm{fr} .50$

PISANI et DIRVELL. La chimie du laboratoire. 1 vol in-12. $1882 \quad 4 \mathrm{fr}$.

QUATREFAGES (de). L'espèce humaine. 1 vol. in-S de la Biblioth. scientif. intern., 6 é édit. 1880 .

$6 \mathrm{fr}$.

QITATREFAGES (de) Charles Darwin et ses précurseurs francais. Elude sur le - transformisme. 1870, 1 vol. in -8 . 5 fr.

RICHE. Manuel de Chinie médicale. 1880,1 rol in-1S avec 200 fig. dans le texte. . 3e édition.

$8 \mathrm{fl}$.

SAPORTA el MARION. L'évolution du règne végétal, les cryptogames 1 vol. in-8 de la Bibliothéque scient. intern., avec 85 fig. dans le texte 6 fr.

SCHMIDT (0). La descendance de l'homme et le darwinisme. 1 vol. in-8 avec figures $3^{\circ}$ édition 1878 . $6 \mathrm{fr}$.

SCHUTZENBERGER. Les fermentations, avec figures dans le texte, $1 \mathrm{rol}$. in -8 de la Biblioth. scient. internat. 3e édit., 1878. Cart. $6 \mathrm{fr}$.

VOGEL. La photographie et la chimie de la lumière. 1 vol. in-8 de la Bibliothéque scient. internat. avec fig. 3 e édit. $6 \mathrm{fr}$.

WURTZ. La théorie atomique. 1 vol in-8 do la Bibliothéque scient. internat. 3 édit., 1880 : $6 \mathrm{ir}$.

\section{OUVRAGISS CLASSIQUES DE BOTANIQUE POUR LES LYGÉE.}

CLASSE DE QUATRIEME - Gours élèmentaire de botanique, nar M. LE: IFovNien, ancien elève de l'École normale supérieure, professenr de botanique à la. Faculté des sciences de Nancy. 1 vol. in-12 cartonné avec 250 figures dans lo texte.

$2 \mathrm{fr} .50$.

Classe de pumosophie, - Mistoire naturelle élémentaire (zoolorie, botanique, géulugie), par le Dr LE Norr, ancien professeur de l'Université. 1 vol: ill-12, avec 251 fig. dans le texte, broché

Classe DE Pillosoruie. - Anatomie et physiologie végétales, par M. LE Monnier. 1 vol: in-12, a vee flgures dans le texte.

Paris. -- A. PАrñt, imprimeur de la Faculté de médecine, rue Monsieur-le-Prince, :l. A. DAYY, successeur. 
. 





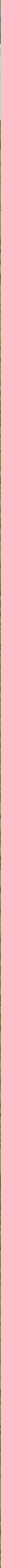


